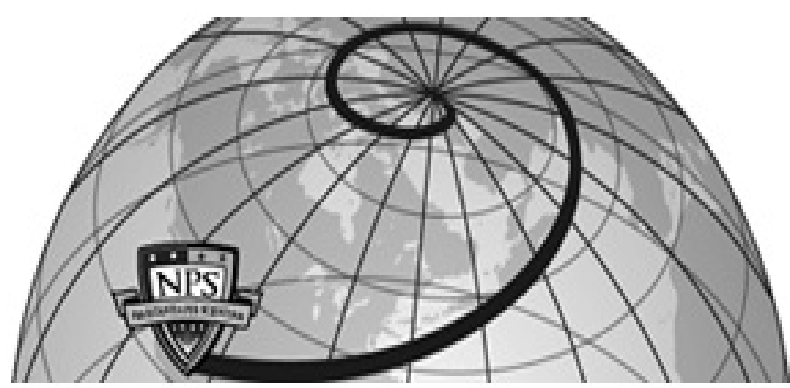

Calhoun: The NPS Institutional Archive DSpace Repository

\title{
An investigation of the effect of acceleration on the burning rate of composite propellants
}

\section{Anderson, James Bruce}

Monterey, California. Naval Postgraduate School

http://hdl.handle.net/10945/12416

This publication is a work of the U.S. Government as defined in Title 17, United States Code, Section 101. Copyright protection is not available for this work in the United States.

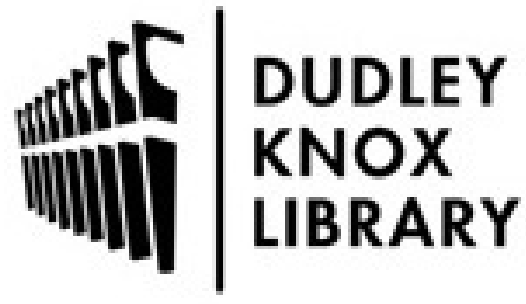

http://www.nps.edu/library
Calhoun is the Naval Postgraduate School's public access digital repository for research materials and institutional publications created by the NPS community. Calhoun is named for Professor of Mathematics Guy K. Calhoun, NPS's first appointed -- and published -- scholarly author.

Dudley Knox Library / Naval Postgraduate School 411 Dyer Road / 1 University Circle Monterey, California USA 93943 


\section{NPS ARCHIVE}

1967

ANDERSON, J.

AN INVESTIGATION OF THE EFFECT

OF ACCELERATION ON THE BURNING RATE

OF COMPOSITE PROPELLANTS

JAMES BRUCE ANDERSON 
LIBRARY

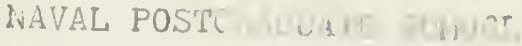

l.:ONTEREY, CALIF. 9SY 
This document has been approved for public release and sale; its distribution is unlimited. 


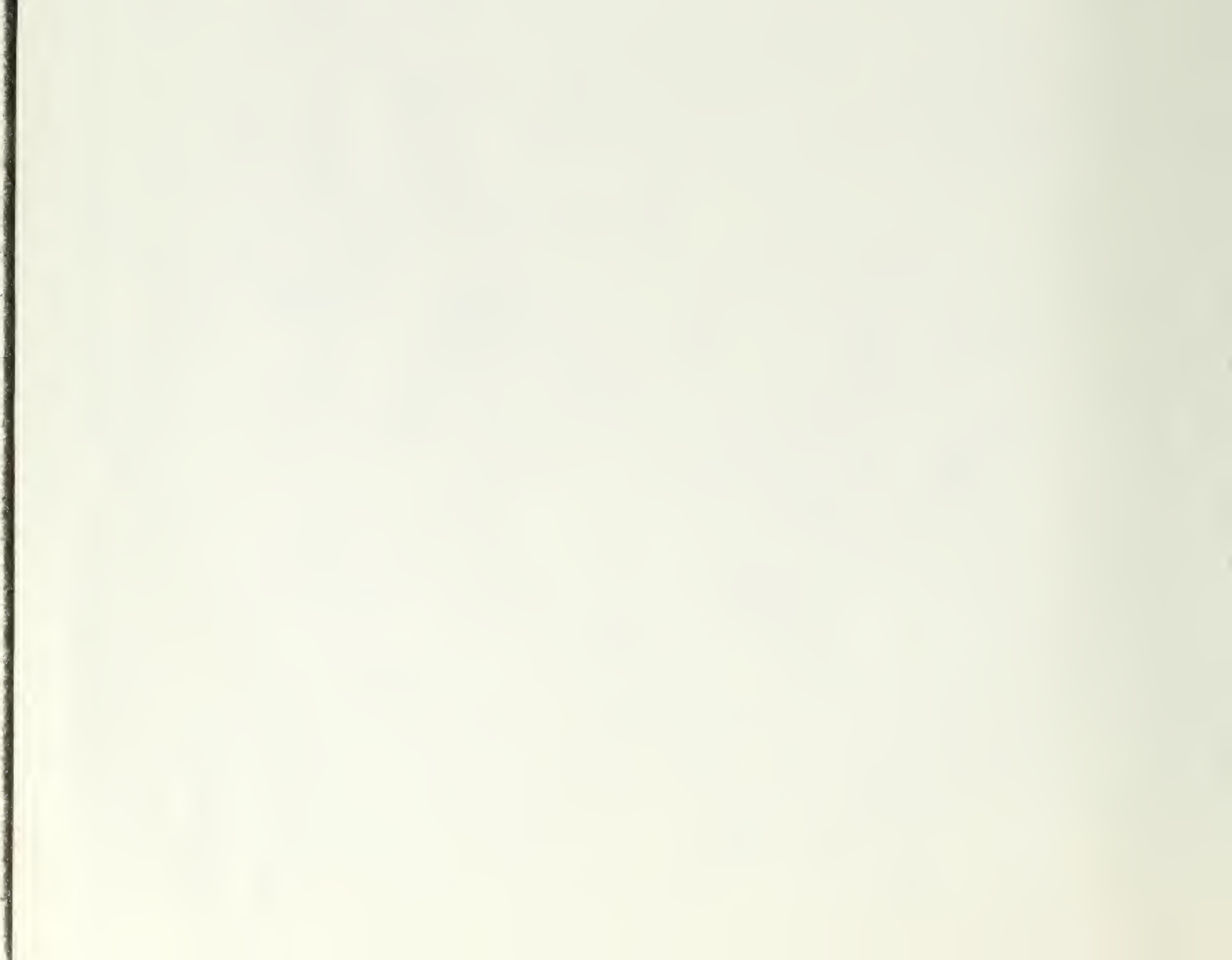


II 



\section{AN INVESTIGATION OF THE EFFECT OF ACCELERATION ON THE BURNING RATE OF COMPOSITE PROPELLANTS}

by

James Bruce Anderson

Lieutenant Commander, Jnited States Navy

B.S., Stanford University, 1956

Submitted in partial fulfillment of the requirements for the degree of DOCTOR OF PHILOSOPHY

from the

UNITED STATES NAVAL POSTGRADUATE SCHOOL August 1966 


\section{ABSTRACT}

The average burning rates of composite solid rocket propellants were measured in acceleration fields up to 2000 times the standard acceleration of gravity. The acceleration vector was perpendicular to and into the burning surface. Propellant strands were burned in a combustion bomb mounted on a centrifuge, and surge tanks were employed to ensure essentially constant pressure burning at 500, 1000 , and 1500 psia.

The burning rates of both aluminized and non-aluminized composite propellants were found to depend on acceleration. The effect of acceleration on burning rate was found to depend on the burning rate of the propellant without acceleration, aluminum mass loading, and aluminum mass median particle size. The relative burning rate increase was found to be greater for slow burning propellants than for faster burning propellants.

The experimental results are compared with two models proposed by other investigators. The results indicate that more complex modeling will be required to explain the observed acceleration effects. 


\section{ACKNOWLEDGMENTS}

There have been many contributors to the successful completion of the research effort reported in this thesis. The writer wishes to express his sincere appreciation to Mr. Norman Walker, Civilian Supervisor of the U.S. Naval Postgraduate School Machine Facility, and his staff, for the construction of the centrifuge; Mr. Edward J. Smith for his assistance in the installation and operation of the centrifuge equipment; Dr. Ralph Anderson, formerly of United Technology Center, Sunnyvale, California, and Mr. James P. Diebold of the Development Engineering Branch, U.S. Naval Ordnance Test Station, China Lake, California, for furnishing the propellant specimens used in the investigation; Drs. John R. Clark and John W. Schultz, of the Department of Material Science and Chemistry, U.S. Naval Postgraduate School, for their help with the propellant residue analyses; and his wife, Nelma, for her assistance in preparation of the manuscript. The writer is particularly indebted to his teacher and advisor, Dr. Roy E. Reichenbach, for his guidance in the conduct and reporting of this research.

Many other people too numerous to mention have made important contributions to this project and it is hoped that the non-appearance of their names will not be misinterpreted as lack of appreciation for their efforts. 

TABLE OF CONTENTS

CHAPTER

PAGE

I. INTRODUCTION . . . . . . . . . . .

II. RECENT INVESTIGATIONS . . . . . . . . 24

III. EXPERIMENTAL EQUIPMENT AND PROCEDURES . . . 43

Equipment . . . . . . . . . 43

Propellant specimens ........ 68

Experimental procedure ........ 81

Data reduction........... . 88

IV. EXPERIMENTAL RESULTS AND DISCUSSION . . • . 96

Burning rate experiments . . . . . . 96

Residue analysis . . . . . . . 157

Comparison of experimental results with proposed theoretical models ..... 168

Summary and discussion . . . . . . 178

V. CONCLUSIONS . . . . . . . . . 190

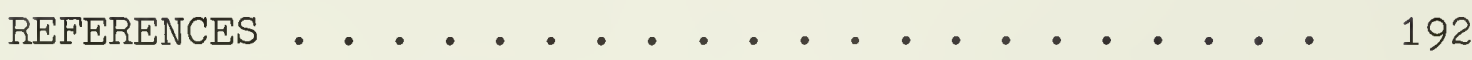

SELECTED BIBLIOGRAPHY • . . . . . . . . . 194

APPENDIX I . . . . . . . . . . . . 197

APPENDIX II ................... 200 



\section{LIST OF TABLES}

TABLE

PAGE

I. X300 Series AP Size Distribution . . . . . . 76

II. Burning Rate Summary X104 Propellant 500 psia. . 152

III. Burning Rate Summary X304 Propallant 500 psia. . 153 



\section{LIST OF PLATES}

PLATE

PAGE

1. 76 inch Diameter Centrifuge Facility, U.S. Naval Postgraduate School, Monterey, California. . . 44

2. Centrifuge Control Station . . . . . . . . 46

3. Centrifuge Rotor . . . . . . . . . . . 48

4. Combustion Bomb - Surge Tank System

(Rotor System) . . . . . . . . . . 50

5. Strand Holders Used with Electric Timers in

Burning Rate Experiments . . . . . . . . 51

6. Strand Holders Used with Pressure Instrumentation

in Burning Rate Experiments . . . . . . . . 52

7. Strand Holder and Bomb Assembly . . . . . . . 53

8. Centrifuge Lower Shaft Detail . . . . . . . . 56

9. Centrifuge Base Structure . . . . . . . . . 57

10. Pressure Circuit . . . . . . . . . . 59

11. 12 v. d.c. Electrical System . . . . . . . . 61

12. $115 \mathrm{~V}$. a.c. Electrical Circuit. . . . . . . 62

13. Timing, Ignition, and Continuity-Test Circuits . . 63

14. Visicorder Timing Circuit . . . . . . . . . . 64

15. Vibration Pickup Circuit . . . . . . . . . 65

16. 6 v.d.c. Electrical System . . . . . . . 66

17. Jigs for Cutting and Drilling Propellant

Samples . . . . . . . . . . . . . 78 



\section{LIST OF FIGURES}

FIGURE

PAGE

1. Thrust versus Time for Spinning End-Burning . . 18 Rocket Motors

2. Pressure History of TM-3 Rocket Motors in Spin and Nonspin Environment . . . . . . . . 19

3. Predicted Burning Rate Increase with Acceleration Level for Two Particle Size Distributions...

4a. Effect of Radial Acceleration on Burning Rate Increase of a Non-aluminized Propellant

4b. Effect of Radial Acceleration on Burning Rate Increase of Propellant Containing Aluminum

Powder with $8 \mu$ Mass-median Diameter ....

5. Burning Rate Augmentation due to Acceleration

Predicted by Modified Granular Diffusion

Flame Model

6. X100 Series AP Size Distribution . . . . . 70

7. Aluminum Powder Size Distribution . . . . . 71

8. X200 Tri-modal AP Size Distribution . . . . 73

9. Aluminum Powder Size Distribution . . . . . 75

10. Radial Acceleration G at 35.6 inch Radius . . . 92

11. Radial Acceleration $G_{r}$ at 35.6 inch Radius . . 93

12. Angular Displacement of the Resultant

Acceleration Vector $G$ with Changing

Radial Acceleration $\mathrm{G}_{\mathrm{r}}$........... 94

13. Burning Rate versus Acceleration -

X101 Propellant at $500 \mathrm{psia}$. . . . . . 98

14. Burning Rate Augmentation versus Acceleration -

X101 Propellant at $500 \mathrm{psia}$. . . . . .

15. Burning Rate versus Acceleration -

X101 Propellant at 1000 psia . . . . . . 100 
16. Burning Rate Augmentation versus Acceleration X101 Propellant at 1000 psia . . . . . . 101

17. Burning Rate versus Acceleration X101 Propellant at 1500 psia . . . . . . 102

18. Burning Rate Augmentation versus Acceleration X101 Propellant at 1500 psia. . . . . . . 103

19. Summary of Burning Rate versus Acceleration for X101 Propellant . . . . . . . . . 104

20. Summary of Burning Rate Augmentation versus Acceleration for X101 Propellant . . . . . 105

21. Burning Rate versus Acceleration X102 Propellant at 1500 psia......... 106

22. Burning Rate Augmentation versus Acceleration X102 Propellant at 1500 psia... . . . . 107

23. Burning Rate versus Acceleration X103 Propellant at 500 psia . . . . . . . 108

24. Burning Rate Augmentation versus Acceleration X103 Propellant at 500 psia and 1500 psia . . 109

25. Burning Rate versus Acceleration X103 Propellant at 1500 psia. . . . . . . 110

26. Burning Rate Augmentation versus Acceleration X103 Propellant at 1500 psia . . . . . . . 111

27. Summary of Burning Rate versus Acceleration for X103 Propellant . . . . . . . . . 112

28. Burning Rate versus Acceleration X104 Propellant at 500 psia . . . . . .

29. Burning Rate Augmentation versus Acceleration X104 Propellant at 500 psia . . . . . . . 114

30. Burning Rate versus Acceleration X104 Propeliant at 1000 psia..........

31. Burning Rate Augmentation versus Acceleration X104 Propellant at 1000 psia... . . . .

32. Burning Rate versus Acceleration X104 Propellant at 1500 psia . . . . . . . 
33. Burning Rate Augmentation versus Acceleration X104 Propellant at $1500 \mathrm{psia}$........ 118

34. Summary of Burning Rate Augmentation versus Acceleration for X104 Propellant .......

35. Summary of Burning Rate versus Acceleration for X100 Series Propellants at 1500 psia . . . 120

36. Summary of Burning Rate Augmentation versus Acceleration for X100 Series Propellants at $1500 \mathrm{psia}$. . . . . . . . . . 121

37. Burning Rate Increase Ratio versus Acceleration X200 Propellant . . . . . . . . . . 122

38. Burning Rate Augmentation versus Acceleration X301 Propellant at $500 \mathrm{psia}$. . . . . . 123

39. Burning Rate Augmentation versus Acceleration X301 Propellant at $1000 \mathrm{psia}$. . . . . . 124

40. Burning Rate Augmentation versus Acceleration X301 Propellant at $1500 \mathrm{psia}$. . . . . . 125

41. Burning Rate versus Acceleration -

X301 Propellant at 500, 1000 and 1500 psia . . 126

42. Summary of Burning Rate Augmentation for X301 Propellant

43. Burning Rate versus Acceleration X302 Propellant at 1000 psia......... 128

44. Burning Rate Augmentation versus Acceleration X302 Propellant at 1000 psia . . . . . .

45. Burning Rate versus Acceleration X303 Propellant at 1000 psia . . . . . . 130

46. Burning Rate Augmentation versus Acceleration X303 Propellant at $1000 \mathrm{psia}$. . . . . . 131

47. Burning Rate versus Acceleration X304 Propellant at 500 psia . . . . . . 132

48. Burning Rate Augmentation versus Acceleration X304 Propellant at 500 psia ........ 133

49. Burning Rate versus Acceleration X304 Propellant at 1000 psia........ 134 
50. Burning Rate Augmentation versus Acceleration X304 Propellant at 1000 psia........ 135

51. Burning Rate versus Acceleration -

X304 Propellant at 1500 psia . . . . . . 136

52. Burning Rate Augmentation versus Acceleration -

X304 Propellant at 1500 psia . . . . . . . 137

53. Summary of Burning Rate versus Acceleration

for X304 Propellant . . . . . . . . . 138

54. Summary of Burning Rate Augmentation versus

Acceleration for X304 Propellant . . . . . . 139

55. Summary of Burning Rate versus Acceleration

for X300 Series Propellants at 1000 psia. . . 140

56. Summary of Burning Rate Augmentation versus

Acceleration for X300 Series Propellants

at $1000 \mathrm{psia}$. . . . . . . . . . . 141

57. The Effect of Aluminum on Acceleration

Sensitivity of a PBAN Propellant at 1000 psia - 142

58. The Effect of Acceleration on Burning Rate

Pressure Exponent - X100 Series Propellants • • 155

59. The Effect of Acceleration on Burning Rate

Pressure Exponent - X300 Series Propellants • . 156

60. Propellant Residues . . . . . . . . 159

61. Top Views of Propellant Residue . . . . . . . 160

62. Aluminum Retention in Inhibitor Case . . . . . 162

63. X-Ray Diffractometer Charts for Propellant

Residues . . . . . . . . . . . . . . 164

64. X-Ray Diffractometer Charts for Propellant

Residue and Aluminum Oxide. . . . . . . 165

65. X-Ray Diffractometer Chart for Propellant

Residue . . . . . . . . . . . . . 166

66. Propellant Residue Analysis by Infrared

Spectrophotometer 
67. Burning Rate Increase Ratio as a Function of the Acceleration Parameter (G $\mathrm{P} / \mathrm{r}$ ) X104 Propellant ..............

68. Comparison of Glick's Modified Granular Diffusion Flame Model with the Experimental Results for the Non-aluminized X301 Propellant

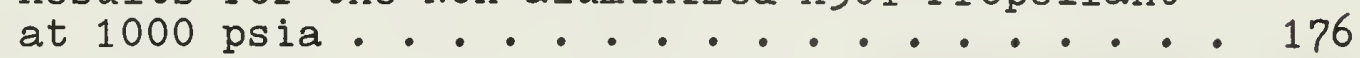

69. Comparison of the Empirical Curves $r / r_{0}=\left(1+K G^{\frac{1}{4}}\right)^{\frac{1}{2}}$ and $r / r_{0}=\left[1+K \cdot\left(G-G_{c}\right)^{0 \cdot 3}\right]^{\frac{1}{2}}$ with the Experimental Results for the Non-aluminized X301 Propellant at 1000 psia . 183

70. Pressure Change at $35^{\prime \prime}$ Radius . . . . . . 203 



\section{CHAPTER I}

\section{INTRODUCTION}

The stability of space probes and rocket assisted projectiles is frequently obtained by spinning the vehicles about their longitudinal axes. In the case of rocket assisted projectiles, where one would like to use existing artillery rifles, spin rates are several thousand RPM. Space probes can usually be stabilized satisfactorily by a spin rate of several hundred RPM.

Early development programs involving spin stabilized vehicles propelled by solid propellant rocket motors showed that static motor firings no longer provided satisfactory predictions of in-flight performance. Telemetered flight data indicated higher combustion chamber pressures, shorter burning time, and lower total impulse. This behavior was found to occur with end-burning grains as well as star shaped and cylindrical port internal burning grains.

As a result of these early observations, subsequent development programs have included spin testing of full size prototype motors. The magnitude of the observed effects is indicated by typical spin test results $[1,2]^{1}$, as shown in Figures 1 and 2. These tests have generally been aimed at determining the prototype performance under the actual spin

${ }^{1}$ Numbers in brackets [] indicate references listed on page 192 . 


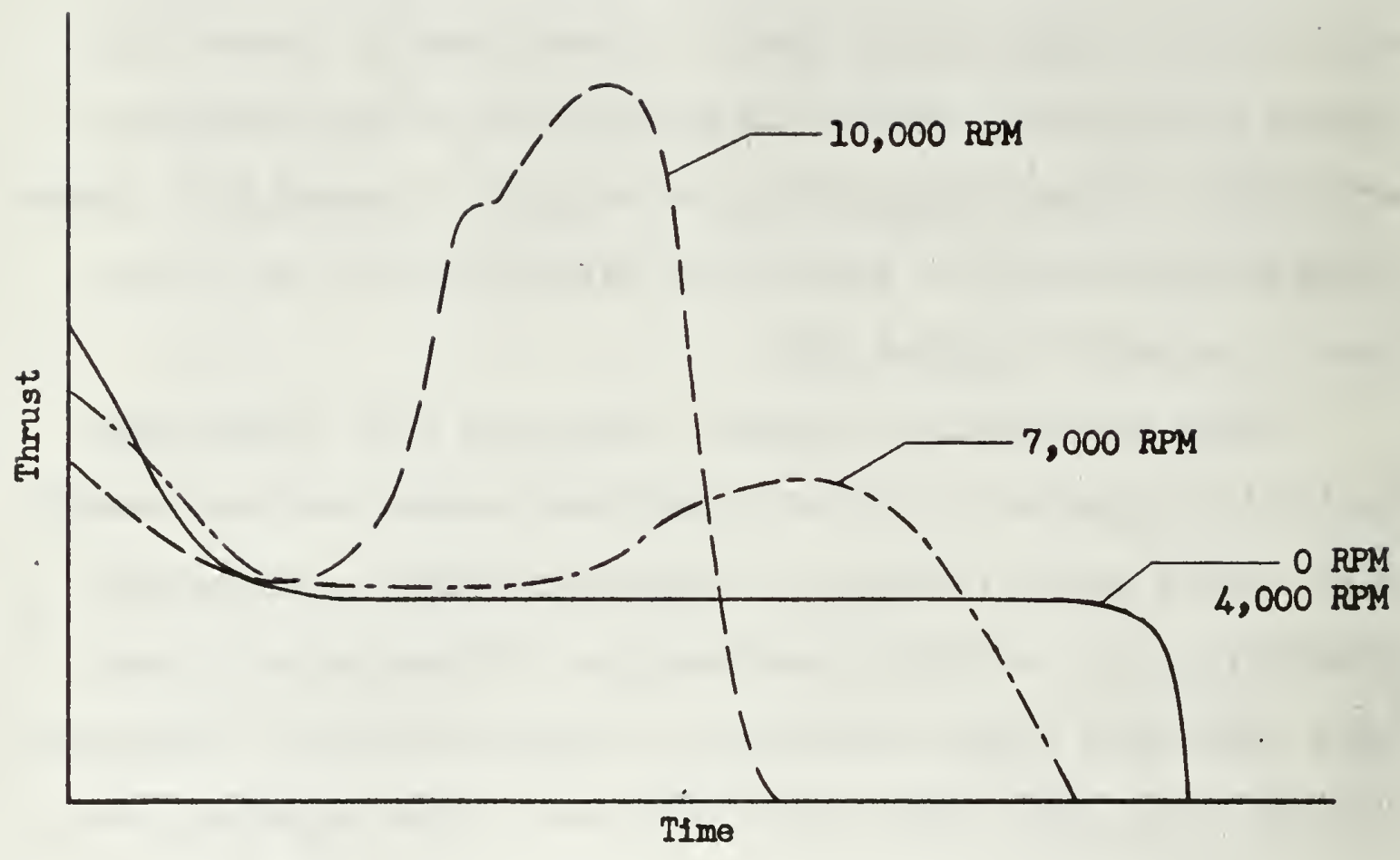

FIGURE 1

THRUST VERSUS TIME FOR SPINNING

END-BURNING ROCKET MOTORS

(VECCHIO AND HARNETT, [1] ) 


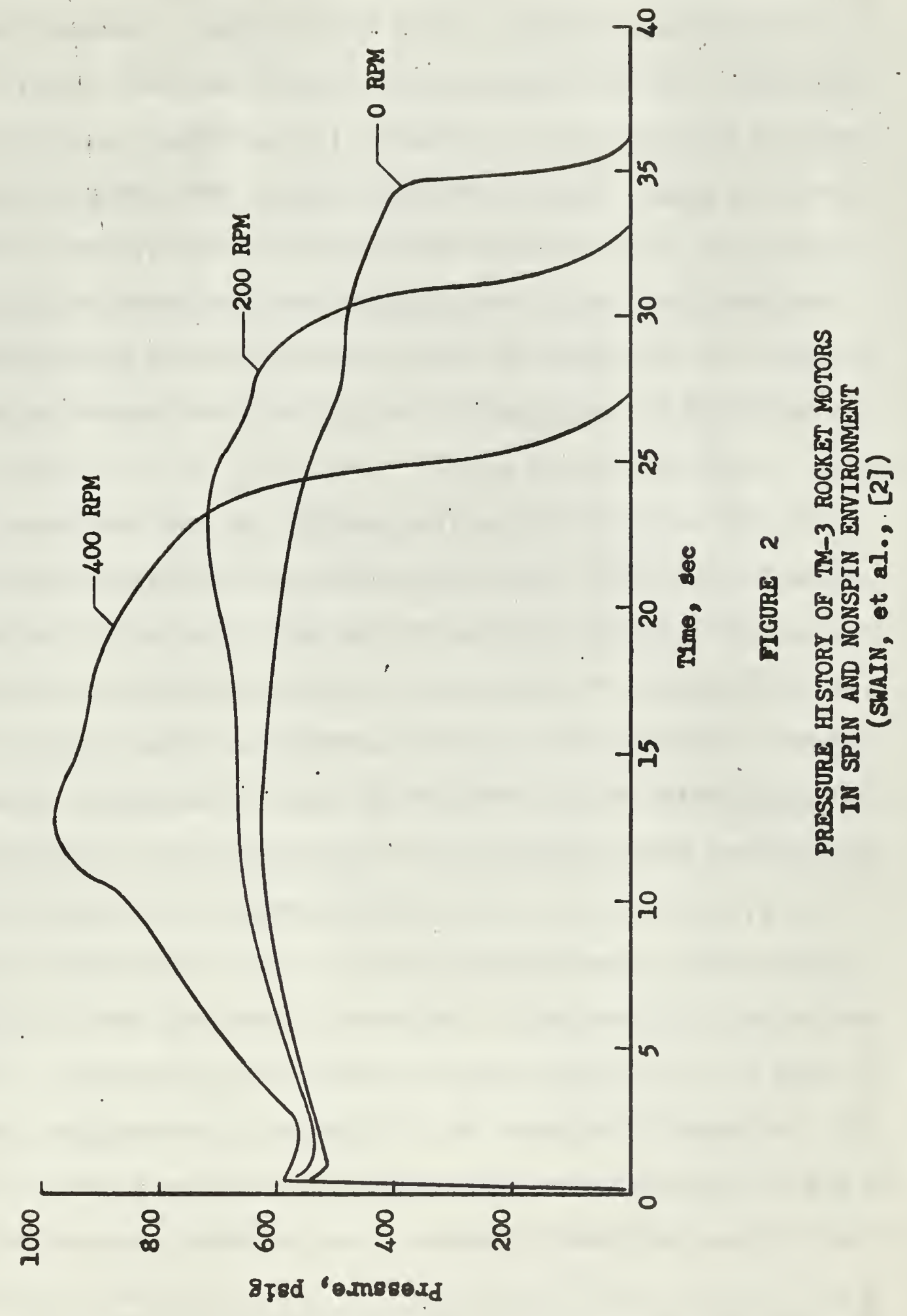


environment and then attempting to reduce performance losses to an acceptable level by changing the propellant composition and/or grain design. The NASA Langley Research Center (NASA-LRC) has two facilities for such testing. Their Rocket Motor Spin Test Apparatus [2] has been used to test fire solid rocket motors weighing up to 2800 pounds with diameters up to 30 inches and lengths to 147 inches. Motor performance has been investigated at spin rates from 100 to 900 RPM. Their Variable Dynamic Force Vector Rocket Test Apparatus [3] is designed to study the combined effects of axial acceleration and spin.

Early work of a more fundamental nature was undertaken by Redel, Inc. [4], in which small end-burning motors were mounted on a centrifuge and fired while subjected to acceleration fields up to 100 times the standard acceleration of gravity. Various solid propellants were investigated and the qualitative results indicate that acceleration does indeed affect the burning rate; the degree being dependent upon the propellant type and the orientation of the acceleration vector to the burning surface. The significance of the results was somewhat obscured however by the deposition of slag in the motor nozzle throat during burning.

The combustion zone in a spinning, end-burning rocket motor is subjected to an acceleration vector parallel to the burning surface. However, the information available to date indicates that the effect of spin on propellant burning rate in end-burning motors may be largely due to 
erosive burning ${ }^{2}$ caused by the formation of a vortex in the motor chamber. The combustion zone in a spinning motor with an internal burning cylindrical port grain is subjected to an acceleration vector perpendicular to the burning surface. In addition, a vortex can be expected in the grain port. The work reported by Redel [4] indicates that the radial acceleration is a significant factor, but it is not known at this time whether vorticity in the grain port also affects the burning rate. If gas dynamics in the grain port is a significant factor, coupling with the acceleration affects may be involved.

In view of the importance of spin effects on the degradation of rocket motor performance and the complete lack of quantitative experimental information it was decided to conduct a careful study of one aspect of the problem. Radial acceleration appeared to be the most probable cause of the burning rate increases observed in spinning motors with internal burning grains and for this reason was chosen as the subject for investigation.

The objectives of the investigation were three-fold. The first objective was to determine the quantitative effect of acceleration on the burning rate of typical solid composite propellants while burning at constant pressure. The second objective was to find the effect of pressure level on the burning rate increase at a given acceleration. The third

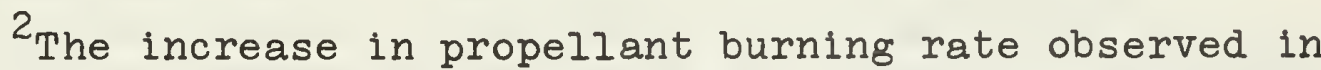
the presence of high velocity gas flow parallel to the burning surface is called erosive burning. 
objective was to find the effect of changing aluminum mass loading and aluminum particle size ${ }^{3}$ on the sensitivity of the propellant burning rate to acceleration. Such information could lead to a better understanding of the mechanisms involved, indicate areas for further research efforts, and serve as a basis for the construction and evaluation of analytical models.

In order to study the effect of acceleration alone on propellant burning rate, it was decided to use conventional strand burning techniques in conjunction with a centrifuge. Such a system was designed and constructed for subjecting propellant strands to centrifugal force fields up to 2000 times the standard acceleration of gravity while burning at preselected pressures from atmospheric to 3000 pounds per square inch. A 1665 cubic inch combustion bomb and surge tank volume ensured essentially constant pressure during burning, and the use of relatively short, two and onequarter inch long, propellant strands at a centrifuge radius of three feet limited the total acceleration change during burning to less than seven per cent of the initial value. Other investigators have also been working on problems associated with spinning rocket motors, and during the course of the present study, investigations have been reported by Northam [5], Crowe, et al. [6], and Glick $[7]$.

${ }^{3}$ Aluminum particle size refers to the mass median diameter of a mixture of particles. Aluminum particle size distributions typical of those used in composite solid propellants may be seen in Figure 7 , page 71 . 
The reported results, including two analytical models, are discussed in Chapter II. The centrifuge and associated equipment used in the present study are described in detail in Chapter III, and the experimental results are presented and discussed in Chapter IV. 


\section{RECENT INVESTIGATIONS}

Three investigations concerned with the effect of acceleration on propellant burning rate have been reported since the present work was begun. The first of these investigations was reported by Northam [5] who measured the burning rate of an aluminized propellant in acceleration fields up to 300G. Northam used the NASA-LRC centrifuge described in Reference 3 to investigate the effect of acceleration on the burning rate of an aluminized composite propellant. The propellant had a $\mathrm{PBAA}^{4}$ binder, ammonium perchlorate oxidizer, and an aluminum fuel additive. A test motor with a slab grain 4 inches wide $x 6$ inches long $x \frac{1}{2}$ inch thick was used. The motor was mounted on the end of the centrifuge arm with the radial acceleration vector at various angles to the burning surface. Acceleration levels were up to $300 \mathrm{G}$ with the acceleration vector directed into, parallel to, away from, and at angles of $30^{\circ}$ and $60^{\circ}$ to the burning surface. Average chamber pressures ranged from 350 to 829 psia. Chamber pressure variations were obtained by using motor nozzles with different throat areas. The results indicate that only acceleration perpendicular to and toward

${ }^{4}$ PBAA refers to a binder material, the composition of which is presently classified. 
the burning surface affects the burning rate. Burning rate increase at $300 \mathrm{G}$ was approximately $30 \%$.

The second investigation was reported by Crowe, et al. [6]. Crowe's work represents the first known attempt at analyzing the effect of acceleration on the burning rate of aluminized composite propellants.

Crowe first considered the effect of the aluminum particles being delayed in their flight away from the surface and thus burning closer to the surface. He divided the strata above the propellant surface into four regions of finite thickness. In region one, the fuel and oxidizer begin to mix and ignition occurs. In region two, the binder and oxidizer reaction takes place. In region three aluminum particle ignition takes place, and in region four, the aluminum combustion takes place. The gaseous reactants and products are treated as perfect gases with constant molecular weight, and the combustion in regions two and four is treated as the addition of heat at a constant rate per unit length. Although ignition takes place in regions one and three, the net heat addition in these regions is assumed to be zero. Assuming the Mach number to be small and hence kinetic energy to be negligible, Crowe then wrote the steady flow, one dimensional energy equation in each of the four regions. The four regions were matched at their boundaries with the requirement that temperatures and temperature gradients be equal. The result was an expression for heat transfer to the unreacted solid propellant in terms of the energy 
Three investigations concerned with the effect of acceleration on propellant burning rate have been reported since the present work was begun. The first of these investigations was reported by Northam [5] who measured the burning rate of an aluminized propellant in acceleration fields up to 300G. Northam used the NASA-LRC centrifuge described in Reference 3 to investigate the effect of acceleration on the burning rate of an aluminized composite propellant. The propellant had a $\mathrm{PBAA}^{4}$ binder, ammonium perchlorate oxidizer, and an aluminum fuel additive. A test motor with a slab grain 4 inches wide $x 6$ inches long $x \frac{1}{2}$ inch thick was used. The motor was mounted on the end of the centrifuge arm with the radial acceleration vector at various angles to the burning surface. Acceleration levels were up to $300 \mathrm{G}$ with the acceleration vector directed into, parallel to, away from, and at angles of $30^{\circ}$ and $60^{\circ}$ to the burning surface. Average chamber pressures ranged from 350 to 829 psia. Chamber pressure variations were obtained by using motor nozzles with different throat areas. The results indicate that only acceleration perpendicular to and toward ${ }^{4} \mathrm{PBAA}$ refers to a binder material, the composition of which is presently classified. 
the burning surface affects the burning rate. Burning rate increase at $300 \mathrm{G}$ was approximately $30 \%$.

The second investigation was reported by Crowe, et al. [6]. Crowe's work represents the first known attempt at analyzing the effect of acceleration on the burning rate of aluminized composite propellants.

Crowe first considered the effect of the aluminum particles being delayed in their flight away from the surface and thus burning closer to the surface. He divided the strata above the propellant surface into four regions of finite thickness. In region one, the fuel and oxidizer begin to mix and ignition occurs. In region two, the binder and oxidizer reaction takes place. In region three aluminum particle ignition takes place, and in region four, the aluminum combustion takes place. The gaseous reactants and products are treated as perfect gases with constant molecular weight, and the combustion in regions two and four is treated as the addition of heat at a constant rate per unit length. Although ignition takes place in regions one and three, the net heat addition in these regions is assumed to be zero. Assuming the Mach number to be small and hence kinetic energy to be negligible, Crowe then wrote the steady flow, one dimensional energy equation in each of the four regions. The four regions were matched at their boundaries with the requirement that temperatures and temperature gradients be equal. The result was an expression for heat transfer to the unreacted solid propellant in terms of the energy 
addicion in regions two and four, the thicknesses of each of the four regions, the physical properties of the gas, and the mass flow rate.

By assuming the thickness of region four to be greater than region two, Crowe showed that the contribution of the aluminum combustion to the surface heat transfer is negligible unless particle ignition takes place only a few microns from the surface.

This result led to a second model in which Crowe assumed that sufficiently large particles remain on the surface until they have burned to a critical size. This critical size is achieved when the aerodynamic drag force is just equal to the particle weight. Until a particle reaches critical size, the energy released during combustion contributes to the heat transfer to the propellant surface. Having burned to less than critical size, the particle leaves the surface. Once a particle leaves the surface its combustion is assumed to contribute nothing to the surface heat transfer rate. Those particles initially less than the critical size leave the surface immediately and hence have no effect on the rate of heat transfer to the propellant.

The energy equation developed by Crowe and based upon the assumptions discussed above, is

$$
\Delta Q=\rho_{S}\left(r-r_{0}\right) h_{V}=\rho_{S} r Z Q f\left(r_{p c} / r_{p m}, \sigma\right)
$$

where

$$
\begin{aligned}
& \Delta Q=\text { additional energy transferred to surface } \\
& \text { (cal/ } \mathrm{cm}^{2} \mathrm{sec} \text { ) }
\end{aligned}
$$




$$
\begin{aligned}
& \rho_{\mathrm{s}}=\text { propeliant density }\left(\mathrm{gm} / \mathrm{cm}^{3}\right) \\
& r=\text { burning rate with acceleration }(\mathrm{cm} / \mathrm{sec}) \\
& r_{0}=\text { burning rate without acceleration }(\mathrm{cm} / \mathrm{sec}) \\
& h_{\mathrm{V}}=\text { heat of vaporization (cal/gm) } \\
& z=\text { mass fraction of aluminum in the propellant } \\
& \mathrm{Q}=\text { energy released by aluminum combustion (cal/gm) } \\
& \mathrm{r}_{\mathrm{pc}}=\text { critical particle radius (cm) } \\
& \mathrm{r}_{\mathrm{pm}}=\text { mass median particle radius (cm) } \\
& \sigma=\text { variance } \\
& \mathrm{f}\left(\mathrm{r}_{\mathrm{pc}} / \mathrm{r}_{\mathrm{pm}}, \sigma\right)=\text { fraction of mass which must be removed } \text { to reduce all particles larger than the } \\
& \text { critical size to the critical size }
\end{aligned}
$$

Equation (1) is an expression for supplemental energy available for transfer to the surface due to acceleration. The rate of heat transfer from the gas phase reaction, which is assumed to be rate controlling in the absence of acceleration, is also assumed to be unaffected by the acceleration field. The mechanism of energy transport to the surface and its possible dependence on acceleration are not considered. The equation predicts that the burning rate increase varies with aluminum mass loading, and it predicts that the burning rate of a non-aluminized propeliant $(z=0)$ will not be affected by acceleration. Stokes flow is assumed for particle drag, hence the burning rate increase is sensitive to pressure only to the extent that gas velocity is affected. Rearranging Equation (1) one can obtain

$$
r / r_{0}=\frac{1}{1-\left(z Q / h_{v}\right) f\left(r_{p c} / r_{p m}, \sigma\right)}
$$


Crowe assumed a log-normal particle distribution and found the function $\mathrm{f}\left(\mathrm{r}_{\mathrm{pc}} / \mathrm{r}_{\mathrm{pm}}, \sigma\right)$. Assuming the burning rate to increase by a factor of two when all particles burn completely on the surface, the factor $\left(z_{Q} / h_{V}\right)$ equals one-half. The resultant dependence of burning rate on acceleration is shown in Figure 3. Figure 3 shows that if all the particles are the same size, acceleration will have no effect on propellant burning rate up to the critical acceleration. Similarly, with a non-zero variance, acceleration will have no effect until the critical acceleration of the largest particles is reached. The Figure also shows that the assumed maximum burning rate increase is approached faster with a small variance.

Crowe and his co-workers also performed experiments with a test motor spinning about its longitudinal axis. $[6,8]$ The internal burning solid propellant grain had a cylindrical port and tapered ends to provide a constant burning surface area. Spin rates were selected to give radial accelerations at the burning surface up to $114 \mathrm{G}$ at the end of burning, and the centerline nozzle area was chosen to give a nominal static chamber pressure of $600 \mathrm{psia}$. The composite propellants investigated were $\mathrm{PBAN}^{5}$ - ammonium perchlorate (AP) formulations with variations in AP crystal size, aluminum mass loading, and aluminum particle size. No significant change in burning rate was found for the propellant without aluminum. The aluminized

5 PBAN refers to a binder material, the composition of which is presently classified. 


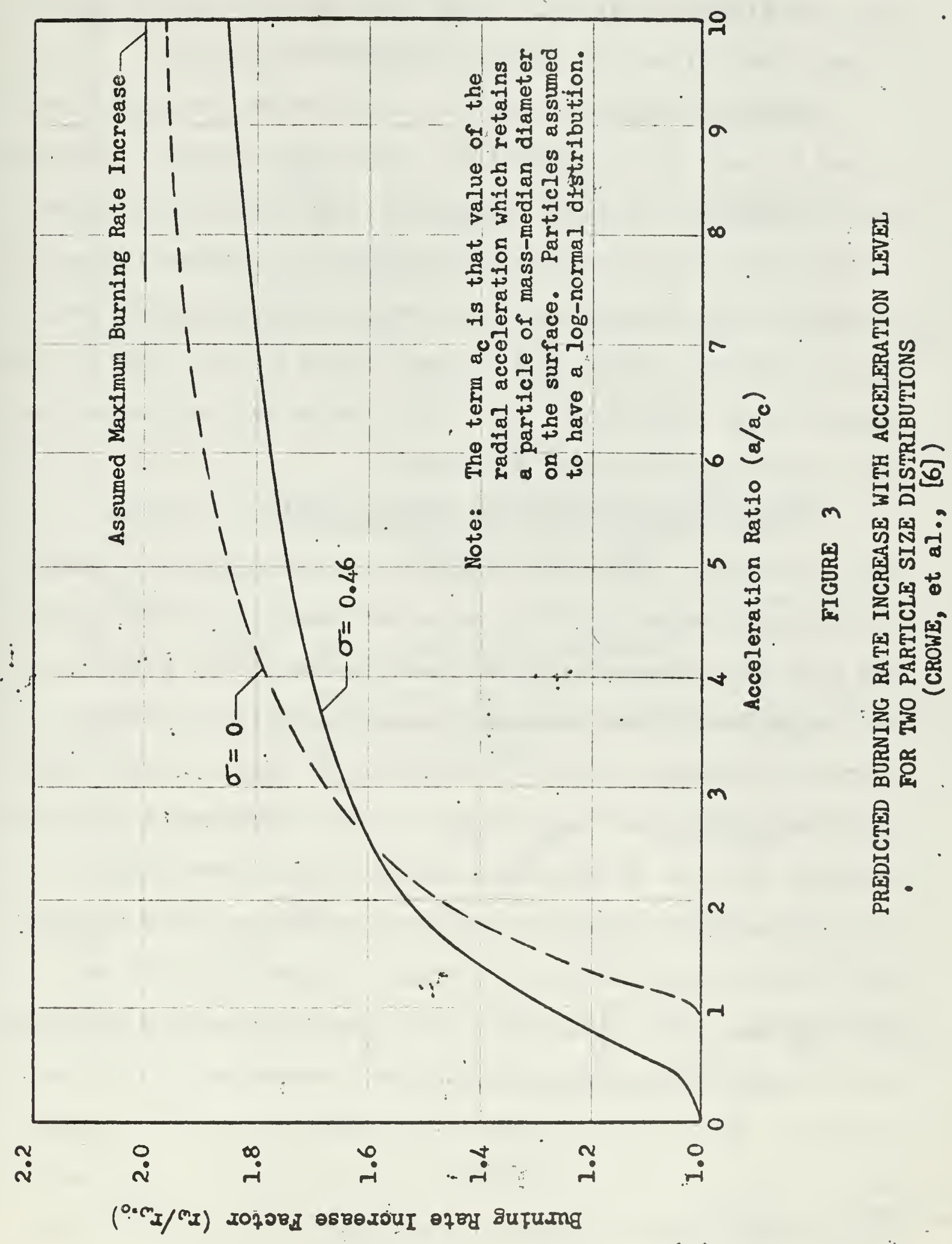


propellants all showed an increase in burning rate, the magnitude depending on AP crystal size, aluminum mass loading, and aluminum particle size. The results for two propellant variations are shown in Figures $4 \mathrm{a}$ and $4 \mathrm{~b}$.

Comparing Figures 3 and $4 \mathrm{~b}$, we see that the trend predicted by the critical particle size model generally agrees with the data. The data indicate a critical acceleration of about 20G, and a maximum burning rate increase factor of at least 1.4 . Figure $4 \mathrm{~b}$ also shows a higher burning rate increase factor at the end of one firing at $30 \mathrm{G}$ than at the beginning of another at 32G. This indicates that acceleration effects may be time dependent.

Several difficulties are encountered in applying Crowe's model. The first question one must ask is: What is the mass median particle size and what is the variance? The size distribution of the aluminum particles added to the propellant during mixing is determinable by several methods. However, there is evidence to indicate that the aluminum particles, once exposed by the regressing binder oxidizer system, do not burn as individual particles.

Crump [9] has taken high speed pictures (4000 frames/ sec) of burning aluminized composite propellants in a one $G$ field. His films show particles of aluminum, exposed by the regressing fuel and oxidizer, accumulating on the surface. That is, the particles tended to stick together, apparently maintaining the same spatial relationship as in the binder. The accumulated aluminum then ignited, fused 


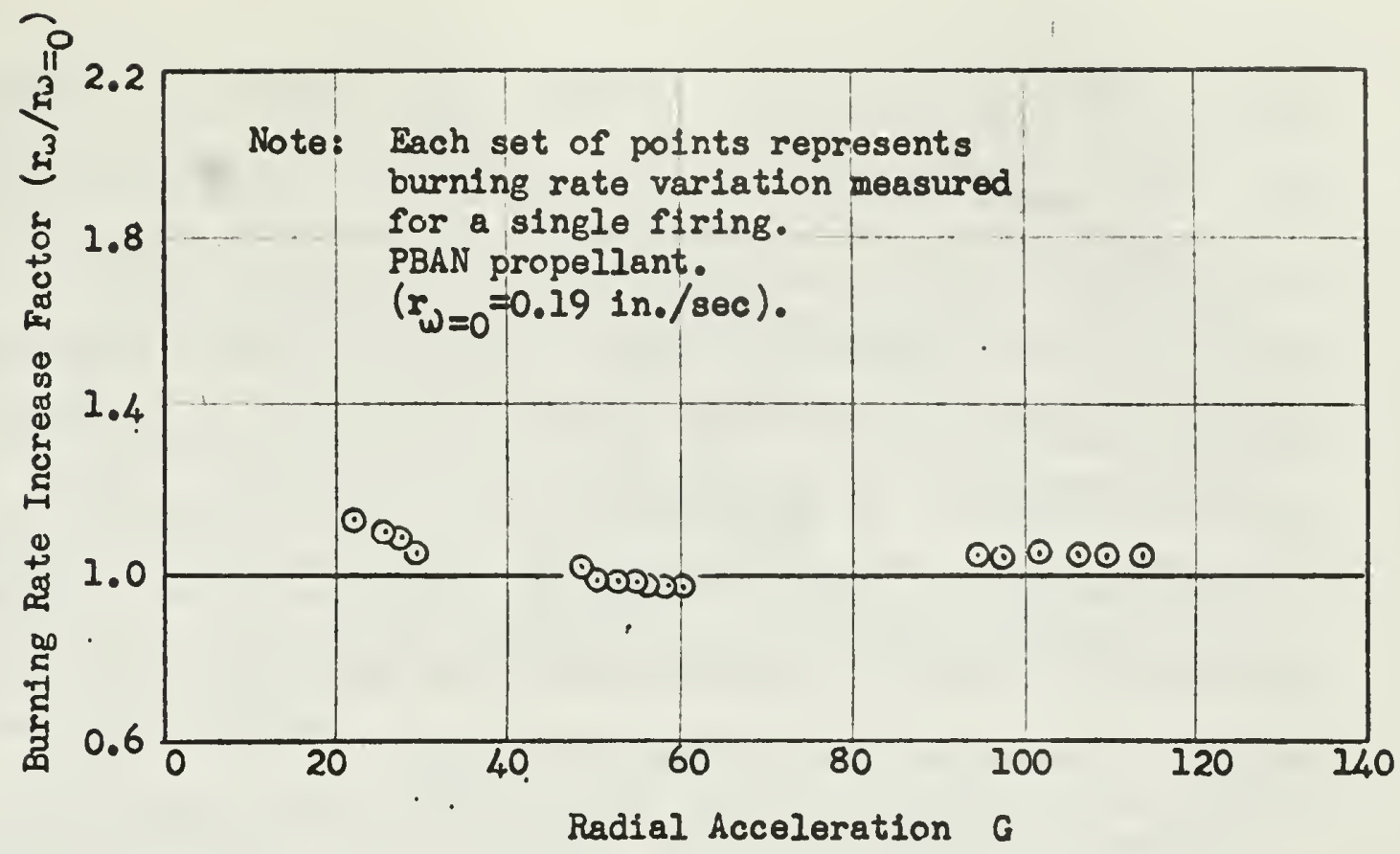

FIGURE $4 a$

EFFECT OF RADIAL ACCELERATION ON BURNING RATE

INCREASE OF A NON-ALUMINIZED PROPEILANT

(CROWE, et al., [6])

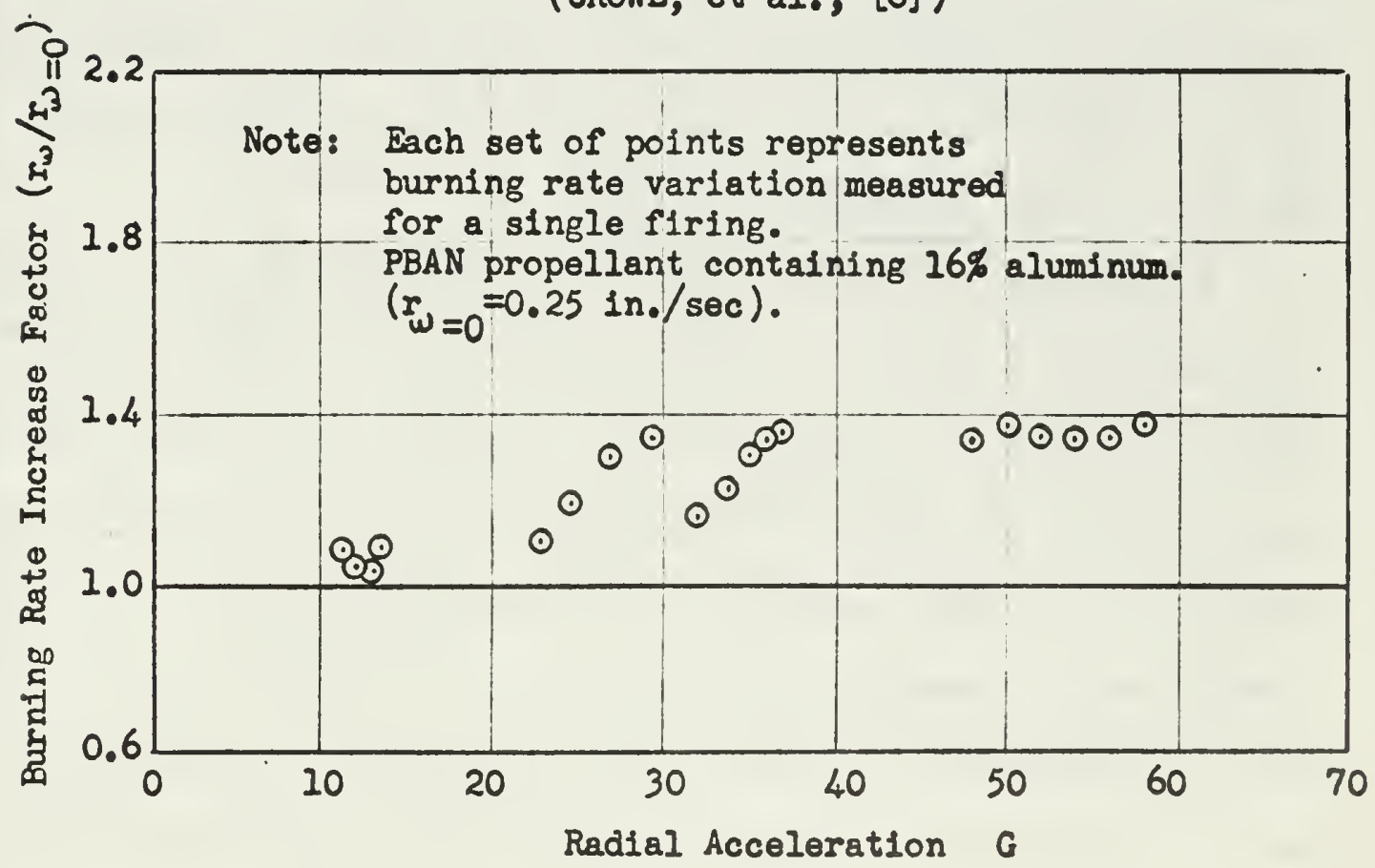

FIGURE $4 \mathrm{~b}$

EFFECT OF RADIAL ACCELERATION ON BURNING RATE INCREASE OF PROPELLANT CONTAINING ALUMINUM POWDER WITH $8-\mu$ MASS-MEDIAN DIAMETER (CROWE, et al., [6]) 
into a ball (agglomerated) and was swept away from the surface. The agglomerates were generally 100 to 300 microns in diameter. Variations were made in binder material, initial aluminum particle size, oxidizer crystal size and aluminum mass fraction. The motion pictures indicate no significant effect on agglomerate size due to changes in binder material or initial aluminum particle size. Decreasing oxidizer crystal size and aluminum mass fraction both tended to decrease the agglomerate size.

Crump's results not only indicate that initial aluminum particle size is not relevant to Crowe's model, but they also suggest that the agglomerate size distribution which is significant to the model is not independent of acceleration. Certainly collision and fusion of agglomerates would be expected as the agglomerate population density increases at the propellant surface.

A more basic difficulty is the assumption that particles greater than the critical size burn on the surface until reaching the critical size, after which they are carried away by the flow. This implies that the products of aluminum/oxidizer combustion are carried away by the flow. Crump's work, however, indicates that at least some of the oxide is deposited on the molten surface of the agglomerates where it collects in the form of a cap. The results reported in the present investigation also indicate that with as little as 8G acceleration, not all of the oxide escapes from the burning surface. As acceleration is increased, 
the amount of oxide swept away by the flow decreases. At several hundred $G$ it appears that a large mass of oxide in the vicinity of the burning surface must be considered.

The second known attempt at analyzing the effect of acceleration on solid propellant burning rate was reported by Glick. [7] Glick proposed an extension to summerfield's granular diffusion flame model [10] to predict the effect of acceleration on the burning rate of non-aluminized composite propellants.

In summerfield's model [10] it is assumed that propellant burning rate is controlled by the rate of energy transfer from the gas-phase reaction to the propellant surface. It is further assumed that the fuel vapor and/or oxidizer vapor is liberated from the solid propellant in the form of pockets. The mass content of these pockets is assumed to be dependent on oxidizer crystal size but not on pressure. At low pressures, chemical reaction rates are assumed to control the burning rate. At high pressures the chemical reaction rates are assumed to be sufficiently high so that the rate controlling mechanism is the diffusion of fuel and oxidizer species into the reaction zones at the pocket boundaries.

The energy balance for heat transfer to the propellant surface by conduction is written

$$
\lambda_{g s}\left[\frac{d T}{d x}\right]_{g s}=\dot{m} c_{S}\left(T_{S}-T_{0}\right)-\dot{m} Q_{S}
$$


where

$$
\begin{aligned}
& \lambda_{\text {gs }}=\text { thermal conductivity of the gas at the } \\
& {\left[\frac{\mathrm{dT}}{\mathrm{dx}}\right]_{\mathrm{gs}}=\begin{array}{l}
\text { temperature gradient in the gas at the } \\
\text { interface }(\mathrm{OK} / \mathrm{cm})
\end{array}} \\
& \dot{\mathrm{m}} \quad=\operatorname{mass} \text { flux }\left(\mathrm{gm} / \mathrm{cm}^{2} \mathrm{sec}\right) \\
& c_{S}=\text { specific heat of the solid (cal } / \mathrm{gm}^{\circ} \mathrm{K} \text { ) } \\
& \mathrm{T}_{\mathrm{S}} \quad=\text { propellant surface temperature }\left({ }^{\circ} \mathrm{K}\right) \\
& \mathrm{T}_{\mathrm{O}}=\text { propellant initial temperature }\left({ }^{\circ} \mathrm{K}\right) \\
& Q_{S} \quad=\text { energy } 1 \text { iberated during exothermic } \\
& \text { decomposition of fuel and oxidizer (cal/gm) }
\end{aligned}
$$

The temperature gradient $(\mathrm{dT} / \mathrm{dx}) \mathrm{gs}$ is approximated by

$$
\left[\frac{d T}{d x}\right]_{g s} \simeq\left(T_{1}-T_{S}\right) / L
$$

where $\mathrm{T}_{1}\left({ }^{{ }} \mathrm{K}\right)$ is the flame temperature and $\mathrm{L}(\mathrm{cm})$ is the distance from the propellant surface to the point where the gas phase chemical reactions are complete (flame thickness). The flame thickness $I=\left(\dot{m} / \rho_{g}\right) \tau$ where $\rho_{g}\left(g m / \mathrm{cm}^{3}\right)$ is the gas density and $\tau$ (sec) is the lifetime of a fuel pocket. At high pressure where diffusion is assumed to be rate controlling, the lifetime $\tau$ is proportional to $\mathrm{d}^{2} / \mathrm{D}$ where $\mathrm{d}(\mathrm{cm})$ is the average diameter of a fuel pocket and $D\left(\mathrm{~cm}^{2} / \mathrm{sec}\right)$ is the binary diffusion coefficient.

Combining Equations ( 3 ) and (4),

$$
r=\frac{\lambda_{g S}\left(T_{1}-T_{S}\right) / L}{\rho_{S}\left[c_{S}\left(T_{S}-T_{O}\right)-Q_{S}\right]}
$$

where $r=$ propellant burning rate $(\mathrm{cm} / \mathrm{sec})$

$$
\rho_{\mathrm{S}}=\text { density of solid propellant }\left(\mathrm{gm} / \mathrm{cm}^{3}\right)
$$


Glick extended Summerfield's model to account for the effect of acceleration on the flame height L. He assumed the fuel gas to be more dense than the oxidizer gas, so that in the presence of an acceleration field the buoyancy force would tend to produce relative motion between the fuel pockets and the surrounding oxidizer gas. Glick considered the effect of this relative motion on convective mass transport and the velocity with which a fuel pocket would move away from the surface.

The only acceleration dependent parameter on the right hand side of Equation (5) is L. Hence the burning rate augmentation may be written

$$
r / r_{0}=L_{0} / L
$$

where the subscript (o) denotes the absence of acceleration. The flame height I may be written

$$
\mathrm{L}=\mathrm{U}_{\mathrm{f}} \tau
$$

where

$$
\begin{aligned}
U_{f}= & \text { component of fuel pocket velocity normal } \\
& \text { to surface }(\mathrm{cm} / \mathrm{sec}) \\
\tau= & \text { pocket lifetime }(\mathrm{sec})
\end{aligned}
$$

With no acceleration, the fuel pocket velocity equals the mass average velocity $r_{0} P_{S} / P_{g}$, and the pocket lifetime $\tau$ is proportional to $d^{2} / D$, hence

$$
L_{0} \propto r_{0} \rho_{s} d^{2} / \rho_{g} D
$$


With acceleration, the fuel pocket velocity component $U_{f}$ is

$$
U_{f}=U_{g}-\Delta U \cos \theta
$$

where

$$
\begin{aligned}
U_{\mathrm{g}}= & \text { mass average gas velocity }(\mathrm{cm} / \mathrm{sec}) \\
\Delta \mathrm{U}= & \text { relative velocity between fuel pocket and } \\
& \text { oxidizer (cm/sec) } \\
\Theta= & \text { angle between the acceleration vector and an } \\
& \text { inward-drawn normal to the propellant surface }
\end{aligned}
$$

The pocket lifetime $\tau$ is reduced by the convective mass transport. The rate of convective mass transfer $m$ is

$$
\mathrm{m}=\mathrm{h}_{\mathrm{D}} \mathrm{A} \Delta \mathrm{C}_{\mathrm{V}}=\epsilon / \tau
$$

where

$$
\begin{aligned}
h_{D} & =\text { convective mass transfer coefficient }(\mathrm{cm} / \mathrm{sec}) \\
A & =\text { surface area }\left(\mathrm{cm}^{2}\right) \\
\Delta C_{\mathrm{V}} & =\text { concentration difference }=\rho_{f} \\
\rho_{\mathrm{f}} & =\text { density of fuel gas }\left(\mathrm{gm} / \mathrm{cm}^{3}\right) \\
\epsilon & =\text { mass of fuel pocket }(\mathrm{gm})
\end{aligned}
$$

Using Equation (9) Glick obtained for the particle lifetime,

$$
\tau \propto\left(d^{2} / D\right)(1 / S h)
$$

The Sherwood number $S h=h_{D} d / D$ and is the ratio of the convective mass transport to the diffusive mass transport.

Equations (8) and (10) combine to give

$$
I=U_{f} \tau \propto\left(U_{g}-\Delta U \cos \theta\right)\left(d^{2} / D\right)(1 / S h)
$$


From Equations (6), (7), and (11),

$$
r / r_{0}=\left(r_{0} \rho_{s} / \rho_{g}\right) /\left[\left(U_{g}-\Delta U \cos \theta\right)(1 / S h)\right]
$$

To find $\left(U_{g}-\Delta U \cos \theta\right)$ Glick assumed the fuel pocket buoyancy to be balanced by the drag force. For the fuel pocket drag coefficient he used $C_{D}=16 /$ Re which Redfield and Houghton [11] showed to apply to gas bubbles rising through a liquid at low Reynolds numbers. Using this value of $C_{D}$, the following expression is obtained for the effect of acceleration (a) on fuel pocket velocity.

$$
\left(U_{g}-\Delta U \cos \theta\right)=r \rho_{s} / \rho_{g}-a d^{2} \Delta \rho(\cos \theta) / 16 \mu_{g}
$$

$a=$ acceleration $\left(\mathrm{cm} / \mathrm{sec}^{2}\right)$ and $\mu_{g}=$ gas viscosity $(\mathrm{gm} / \mathrm{cm} \mathrm{sec})$ For the Sherwood number Sh, Glick used the form

$$
S h=C_{1}+C_{2}(S c R e)^{\frac{1}{2}}=C_{1}+C_{2}(\Delta U d / D)^{\frac{1}{2}}
$$

Substitution of $\Delta U$ from Equation (13) into (14) gives

$$
\mathrm{Sh}=\mathrm{C}_{1}+\mathrm{C}_{2}\left(\mathrm{ad}^{3} \Delta \rho / \mu_{\mathrm{g}} \mathrm{D}\right)^{\frac{1}{2}}
$$

Combining Equations (12), (13), and (15) yields the final expression for $r / r_{0}$. Glick obtained

$$
\begin{aligned}
r / r_{0}= & C_{3}\left(G r / R_{0}\right) \cos \theta+ \\
& {\left[C_{3}^{2}\left(G r / R e_{0}\right)^{2} \cos ^{2} \theta+C_{4}(G r S c)^{\frac{1}{2}}+1\right]^{\frac{1}{2}} }
\end{aligned}
$$

where

$$
\mathrm{Gr}=a \epsilon \Delta \rho \rho_{\mathrm{g}} / \mu_{\mathrm{g}}^{2} \rho_{\mathrm{f}}
$$




$$
\begin{aligned}
& \mathrm{Sc}=\mu_{\mathrm{g}} / \rho_{\mathrm{g}} \mathrm{D} \\
& \mathrm{Re}_{\mathrm{O}}=\rho_{\mathrm{S}} \epsilon^{1 / 3} r_{0} / \mu_{\mathrm{g}} \rho_{\mathrm{f}}^{1 / 3}
\end{aligned}
$$

Glick pointed out that the model ceases to apply as $a \rightarrow \infty$, because $\lim _{a \rightarrow \infty} r / r_{0}=\infty$ and because at sufficiently high acceleration the fuel gas will not escape from the surface. He also stated that acceleration effects should be insensitive to pressure. It is agreed that $S c$ and $R_{0}$ are sensitive to pressure ${ }^{6}$ only through the viscosity $\mu_{\mathrm{g}}$. However, it would appear that $\mathrm{Gr}$ is directly proportional to pressure since gas density appears twice in the numerator and only once in the denominator. Hence $r / r_{0}$ should increase with pressure.

Glick also pointed out that reducing oxidizer particle size will reduce the burning rate augmentation $r / r_{0}$. This is in agreement with the results for metallized propellants reported by Crowe [8] and in Chapter IV of this work.

It can be shown that the combination of Equations (12), (13), and (15) with appropriate manipulation results in the following expression for $r / r$ 。

$$
\begin{aligned}
r / r_{0}= & \left(\mathrm{Gr} / 32 \mathrm{Re}_{0}\right) \cos \theta+ \\
& {\left[\left(\mathrm{Gr} / 32 \mathrm{Re}_{0}\right)^{2} \cos ^{2} \theta+\mathrm{C}_{1}+\mathrm{C}_{2} \mathrm{Gr}^{\frac{1}{2}} \mathrm{Sc}^{\frac{1}{2}}\right]^{\frac{1}{2}} }
\end{aligned}
$$

The boundary condition on Equation (17) is that at $a=0, r / r_{0}=1$. Hence $C_{1}=1$. Rewriting the first term

$6_{\text {The }}$ granular diffusion flame model predicts $r_{0}$ to vary as $\mathrm{P}^{1 / 3}$ at high pressure. [10] 
of Equation (17) in terms of the problem variables,

$$
\left(\mathrm{Gr} / 32 \operatorname{Re}_{\mathrm{o}}\right) \cos \theta=\frac{\mathrm{a}\left[\mathrm{d}^{2}\left(\rho_{\mathrm{f}} / \rho_{\mathrm{g}}-\rho_{\mathrm{o}} / \rho_{\mathrm{g}}\right]\right] \rho_{\mathrm{g}}^{2}}{32 \mu_{\mathrm{g}} \rho_{\mathrm{s}} \mathrm{r}_{\mathrm{o}}} \cos \theta \equiv \phi
$$

All the variables in $\phi$ can be reasonably estimated with the exception of $\left[d^{2}\left(\rho_{f} / \rho_{g}-\rho_{o} / \rho_{g}\right)\right]$ Equation (17) becomes

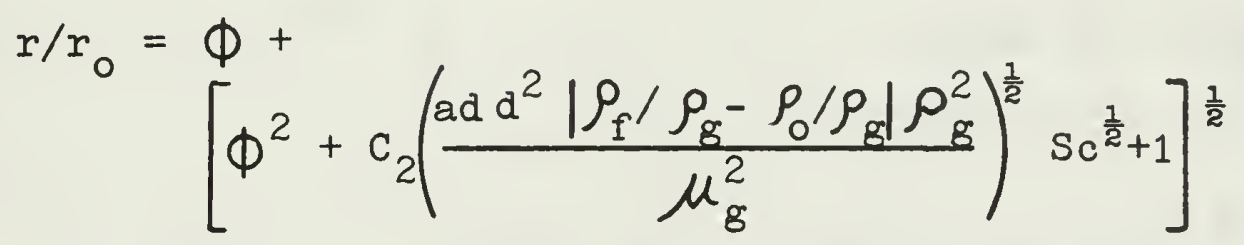

The absolute value sign $\left|\rho_{f} / \rho_{g}-\rho_{0} / \rho_{g}\right|$ appears in Equation (18) because convective mass transport depends on the magnitude only of the relative motion and not the direction of relative motion. The terms involving $\phi$ in Equation (18) are relative motion terms expressing the effect of acceleration on the fuel pocket velocity. The term involving $\mathrm{C}_{2}$ in Equation (18) is the mass transport term.

An important unknown quantity in Equation (18) is the fuel pocket dimension d. Since 100 microns is generally thought to be the order of the flame height $I$ for typical composite propellants, the fuel pocket dimension $d$ is probably no more than 50 microns. The relative densities of the fuel and oxidizer gases are also important, but the composition of binder gasification products is not known at this time.

To show the effect of acceleration on a typical nonaluminized composite propellant as predicted by Equation (18), 
the variables are assigned the following values.

$$
\begin{aligned}
\mathrm{r} & =1.08 \mathrm{~cm} / \mathrm{sec} \\
\mathrm{P} & =1000 \mathrm{psia}=70.3 \mathrm{Kg} / \mathrm{cm}^{2} \\
\mathrm{R}_{\mathrm{O}} & =8.47 \mathrm{x} 10^{4} \mathrm{gm} \mathrm{cm} / \mathrm{gm} \mathrm{mole} \mathrm{O}_{\mathrm{K}} \\
\mathrm{W} & =22 \mathrm{gm} / \mathrm{gm} \mathrm{mole} \\
\mathrm{T} & =2100^{\circ} \mathrm{K} \\
\rho_{\mathrm{S}} & =1.6 \mathrm{gm} / \mathrm{cm}^{3} \\
\mathrm{~d} & =50 \mathrm{microns} \\
\theta & =0 \\
\mu_{\mathrm{g}} & =5.5 \mathrm{x} 10^{-4} \mathrm{gm} / \mathrm{cm} \mathrm{sec} \\
\rho_{\mathrm{g}} & =25 \times 10^{-4} \mathrm{gm} / \mathrm{cm} \mathrm{sec} \\
\rho_{\mathrm{g}} & =\text { PW/RoT }=8.7 \mathrm{x} 10^{-3} \mathrm{gm} / \mathrm{cm} 3 \\
\mathrm{~W}_{\mathrm{f}} & =\text { molecular weight of fuel gas (gm/gm mol) } \\
\mathrm{W}_{\mathrm{o}} & =\text { molecular weight of oxidizer gas (gm/gm mol) }
\end{aligned}
$$

It is assumed that the fuel gas and oxidizer gas are the same temperature. If the solid propellant is $20 \%$ binder and $80 \%$ oxidizer, it can be shown (see Appendix I) that the assumption $\mathrm{W}_{\mathrm{f}} \simeq 2 \mathrm{~W}_{\mathrm{o}}$ leads to $\left(\rho_{\mathrm{f}} / \rho_{\mathrm{g}}-\rho_{\mathrm{o}} / \rho_{\mathrm{g}}\right)=1$. Then $\phi=6.1 \times 10^{-5} \mathrm{G}$, where $G=a / g_{0}$, and $g_{0}=980 \mathrm{~cm} / \mathrm{sec}^{2}$. Assuming the fuel pocket dimension d to be independent of acceleration, Equation (18) may be written

$$
r / r_{0}=6.1 \times 10^{-5} G+\left[\left(6.1 \times 10^{-5} G\right)^{2}+K G^{\frac{1}{2}}+1\right]^{\frac{1}{2}}
$$

Equation (19) requires only one datum point to predict the burning rate change at other accelerations. If we choose $r / r_{0}=1.36$ at $1500 G$, then $K=1.5 \times 10^{-2}$. The choice of fuel pocket size $d$ and the relative fuel and oxidizer gas 
densities have a large effect on the relative importance of $\Phi$. The coefficient $K$ is also affected, but to a lesser extent.

If we had chosen the fuel pocket dimension to be $20 \mathrm{mi-}$ crons, then $\phi=9.76 \times 10^{-6} \mathrm{G}, \mathrm{K}=2.09 \times 10^{-2}$,

and

$$
r / r_{0}=9.76 \times 10^{-6} G+\left[\left(9.76 \times 10^{-6} G\right)^{2}+2.09 \times 10^{-2} G^{\frac{1}{2}}+1\right]^{\frac{1}{2}}
$$

Equations (19) and (20) are shown in Figure 5.

The burning rate change with acceleration predicted by Glick's model is compared with experimental results of this investigation in Chapter IV. 


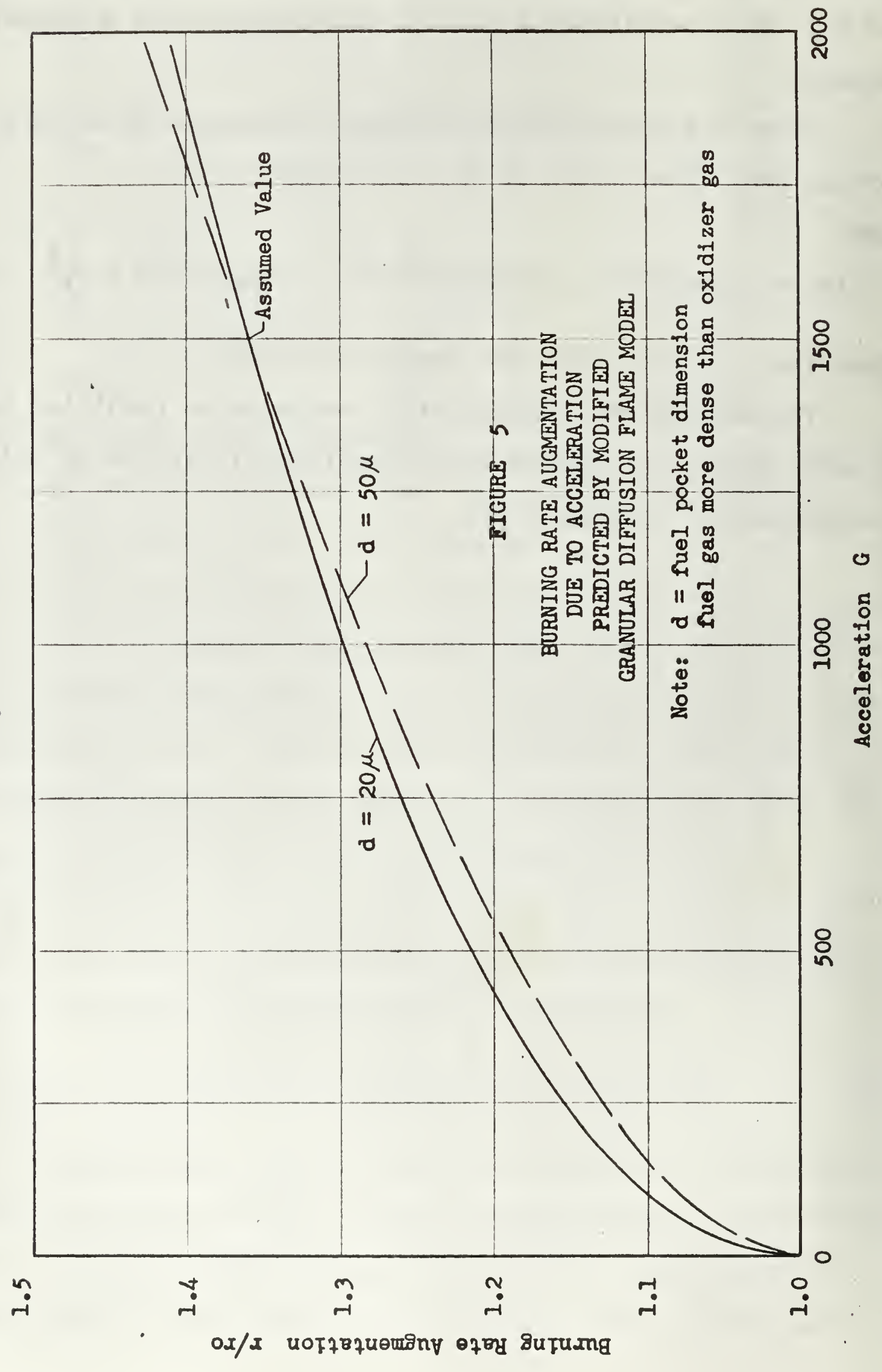




\section{CHAPTER III}

EXPERIMENTAL EQUIPMENT AND PROCEDURES

\section{EQUIPMENT}

\section{General}

The 76 inch diameter centrifuge shown in Plate 1 was designed and constructed at the U.S. Naval Postgraduate School, Monterey, California. The centrifuge was installed in an experimental test cell at the School's Rocket Laboratory. The configuration shown was designed to study the burning rate of solid propellants in acceleration fields up to 2000G. The $1665 \mathrm{cu}$. in. bomb - surge tank system on the rotor was designed for operating pressures up to $3000 \mathrm{psig}$. The centrifuge could be used for other purposes by fitting a suitable rotor to the vertical shaft. The centrifuge capac1ty was $36,000 \mathrm{~g}$-pounds at a maximum speed of $1450 \mathrm{RPM}$.

A 12 channel slip-ring assembly was provided for instrumentation on the rotor. Pressure in the combustion bomb was sensed by a pressure transducer.

Propellant burning rate could be determined by two methods. Three timers were used to record elapsed burning time over known distances, and the total burning time of the sample was determined from the pressure time history recorded on a Visicorder chart. 


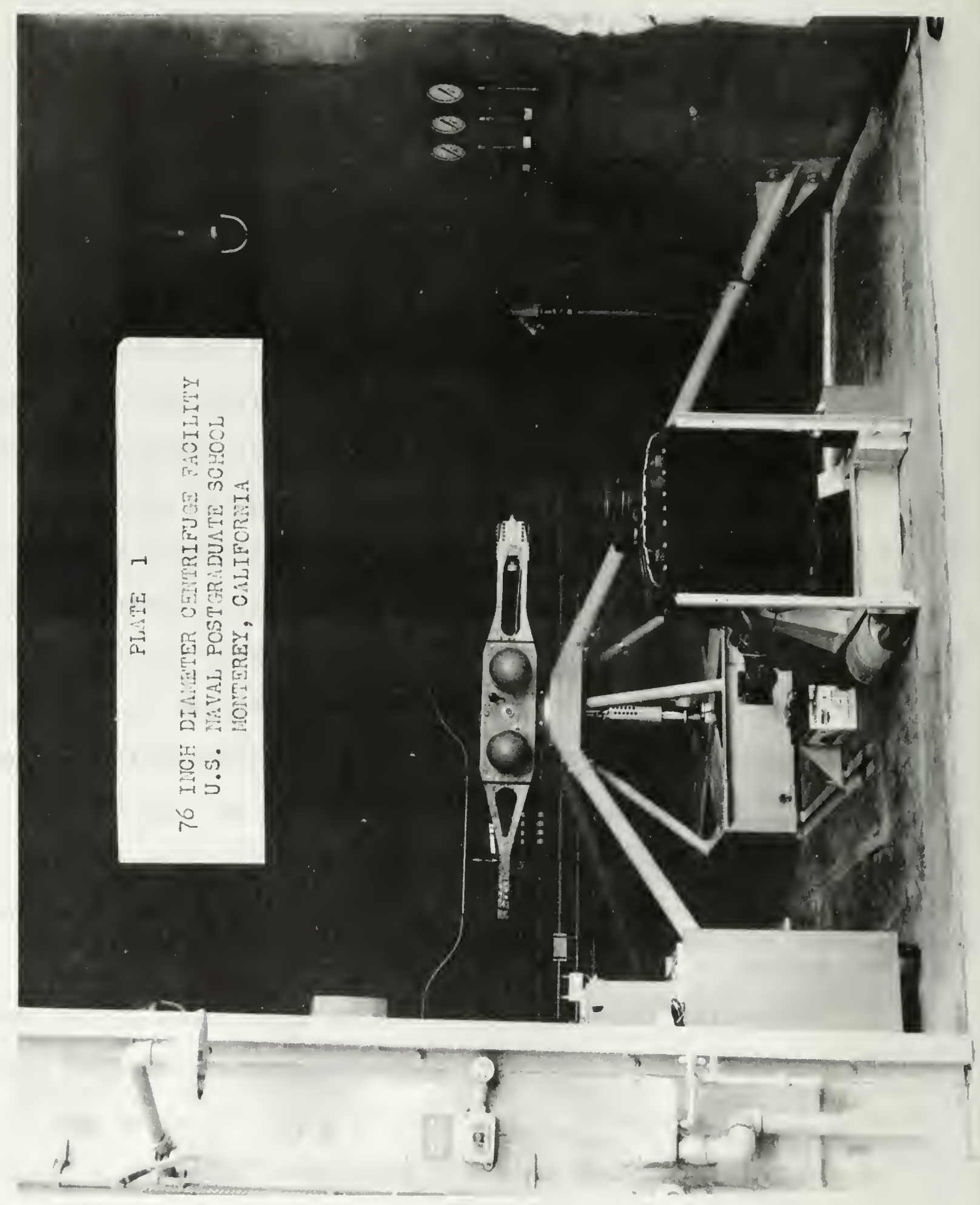


The centrifuge was operated from the remote control station shown in Plate 2.

\section{Centrifuge}

The centrifuge base structure was of welded and bolted construction. The base was constructed from standard 12 inch channel and wide-flange beam stock. The struts supporting the upper bearing housing were standard steel pipe.

The centrifuge shaft rotated in two bearings. The lower bearing was an SKF self-aligning double-row ball bearing. and the upper bearing was an SKF spherical roller bearing (self-aligning). Loads from the lower bearing were transmitted through the base structure to a thrust pad in the floor. Loads from the upper bearing were transmitted to the walls of the test cell through the tubular struts.

The structural parts of the rotor were made of aluminum, and were bolted together with alrcraft quality steel bolts. The rotor arms were constructed from $3 / 4$ inch plate. To these main structural members were secured the combustion bomb, surge tanks and connecting tubes, fittings and valves, pressure transducer, and counterweight.

The rotor assembly was secured to the shaft by a pivot pin. Thus the rotor. was dynamically self-balancing. Static balancing was accomplished by setting the rotor on a balancing stand and adding or removing counterweight plates..

Aerodynamic drag was reduced to an acceptable level by fairings on the rotor arms. 


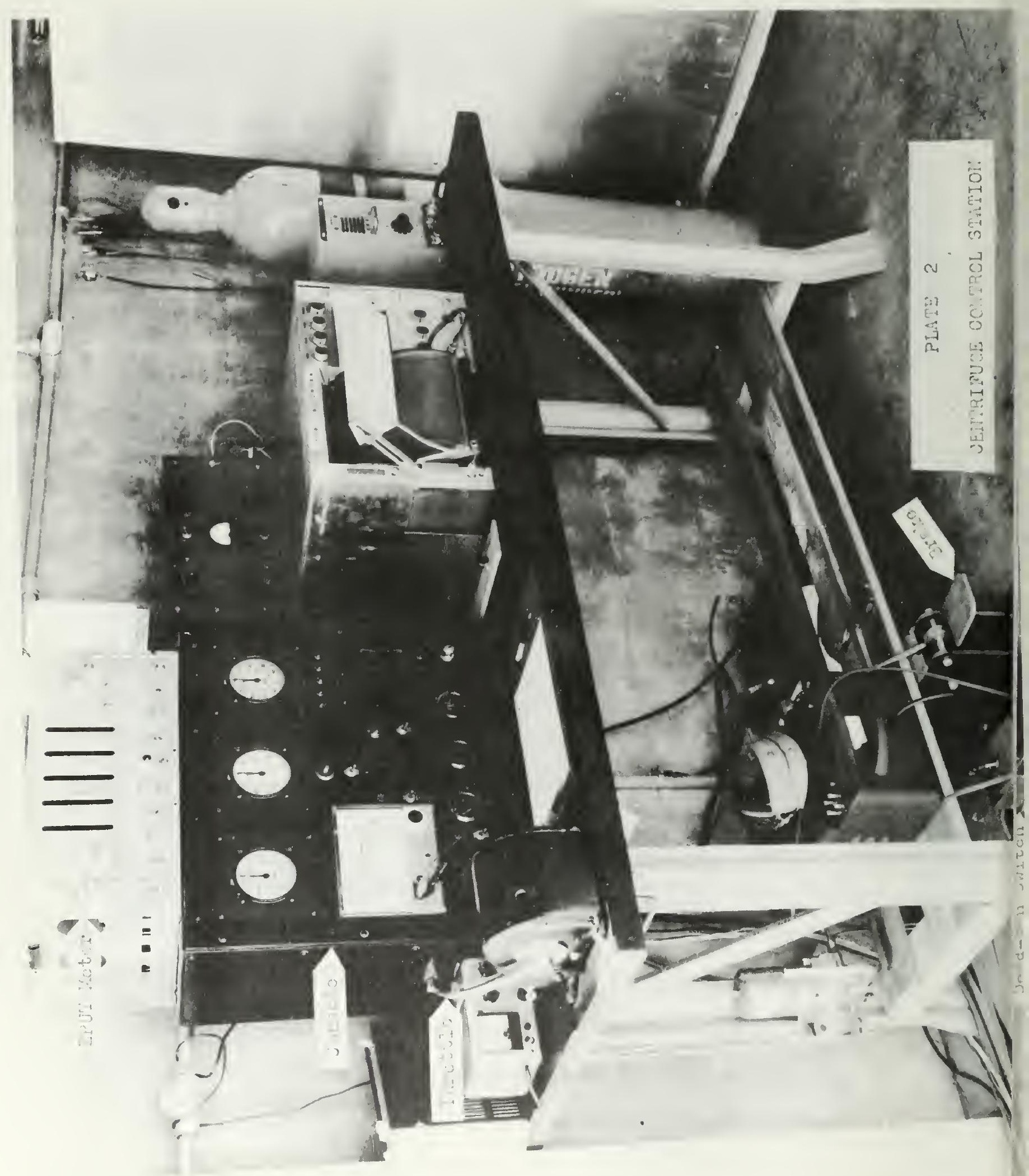


Combustion Bomb - Surge Tank System (Rotor System)

The bomb - surge tank system was wholly contained in the rotor assembly which is shown in Plate 3. It consisted of the combustion bomb, two surge tanks, and connecting tubes and valves. Hereafter, this system will be referred to as the Rotor System.

The combustion bomb was made from three pieces of 321 stainless steel. A hemispherical cap and a reducer section were welded to a cylindrical center section. The reducer section had a $17 / 8$ inch I.D. port to permit insertion of the strand holder. The strand holder also served as a plug and was locked in place with an aluminum collar. The bomb had an inside diameter of 4 inches and a capacity of $115 \mathrm{cu}$. in. It was designed for a working pressure of $3000 \mathrm{psi}$ and was hydrostatically tested to 4500 psi.

The two surge tanks were Navy SCUBA tanks with a capacity of $725 \mathrm{cu}$. in. each. They were made of 6061-T6 aluminum and were designed for a working pressure of 3000 psig. The tanks were lined with Dow Corning Q 92-009 silicone rubber sealant for protection against corrosion.

The tubing used in the system was seamless, annealed 321 stainless steel. The bomb and surge tanks were connected by 0.375 in. O.D. $x 0.258$ in. I.D. tubing. A ballvalve was located between the bomb and the tanks.

The system was pressurized through a quick disconnect fitting located between the ball valve and the surge tanks. 


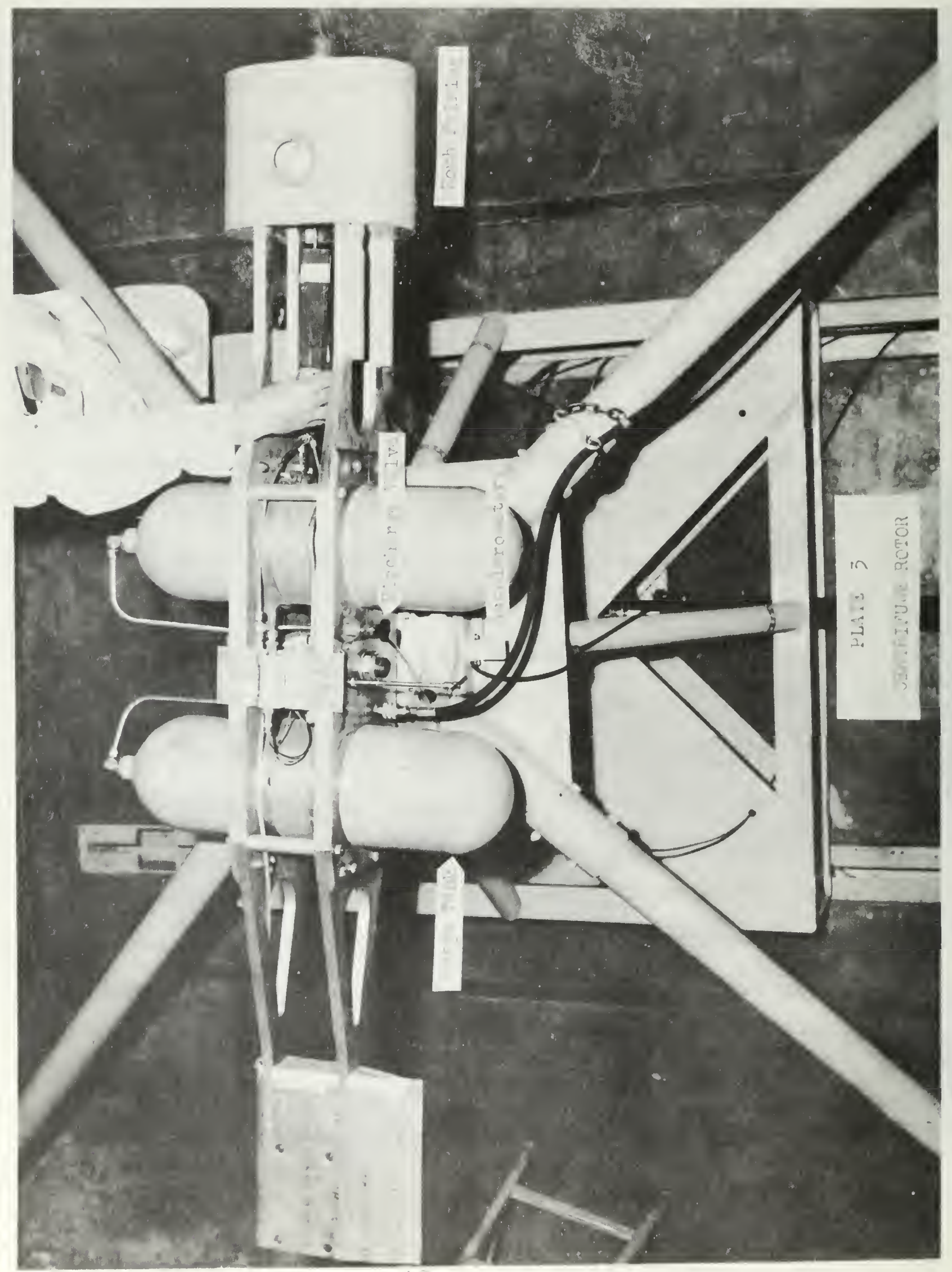


The system could be de-pressurized by a hand operated discharge valve or a solenoid valve actuated by a switch on the control console. Both valves were connected to the bomb by a 0.250 in. O.D. $x 0.180$ in. I.D. tube.

Pressure in the bomb was sensed by a pressure transducer which was connected by a 0.250 in. $0 . D . x 0.180$ in. I.D. tube to a tee in the discharge line.

A diagram of the Rotor System is shown in Plate 4. Strand Holders

The strand holders were all of the same basic type as shown in Plates 5,6 , and 7 . The basic components were a machined aluminum plug, canvas phenolic slab and strand support, insulation sheet, and gland seal. The aluminum plug was a slip fit in the throat of the bomb and the flared edge at the top of the plug was a metal-to-metal fit relative to the bomb throat seat. Thus the strand holder was supported in high radial acceleration fields, and extrusion of the o-ring seal was avoided.

The slab and strand support, both of canvas phenolic, provided structural support for the propellant sample. The slab was fastened to the plug with two \#10-32 FH screws, and the strand support was bonded to the slab with APCO 210 Resin and 180 Hardener. \#6-32 brass machine screws were used for timing and ignition circuit terminals. The terminals were connected to an Amphenol plug by \#20 enameled copper magnet wire. The copper wires were bonded to the back 


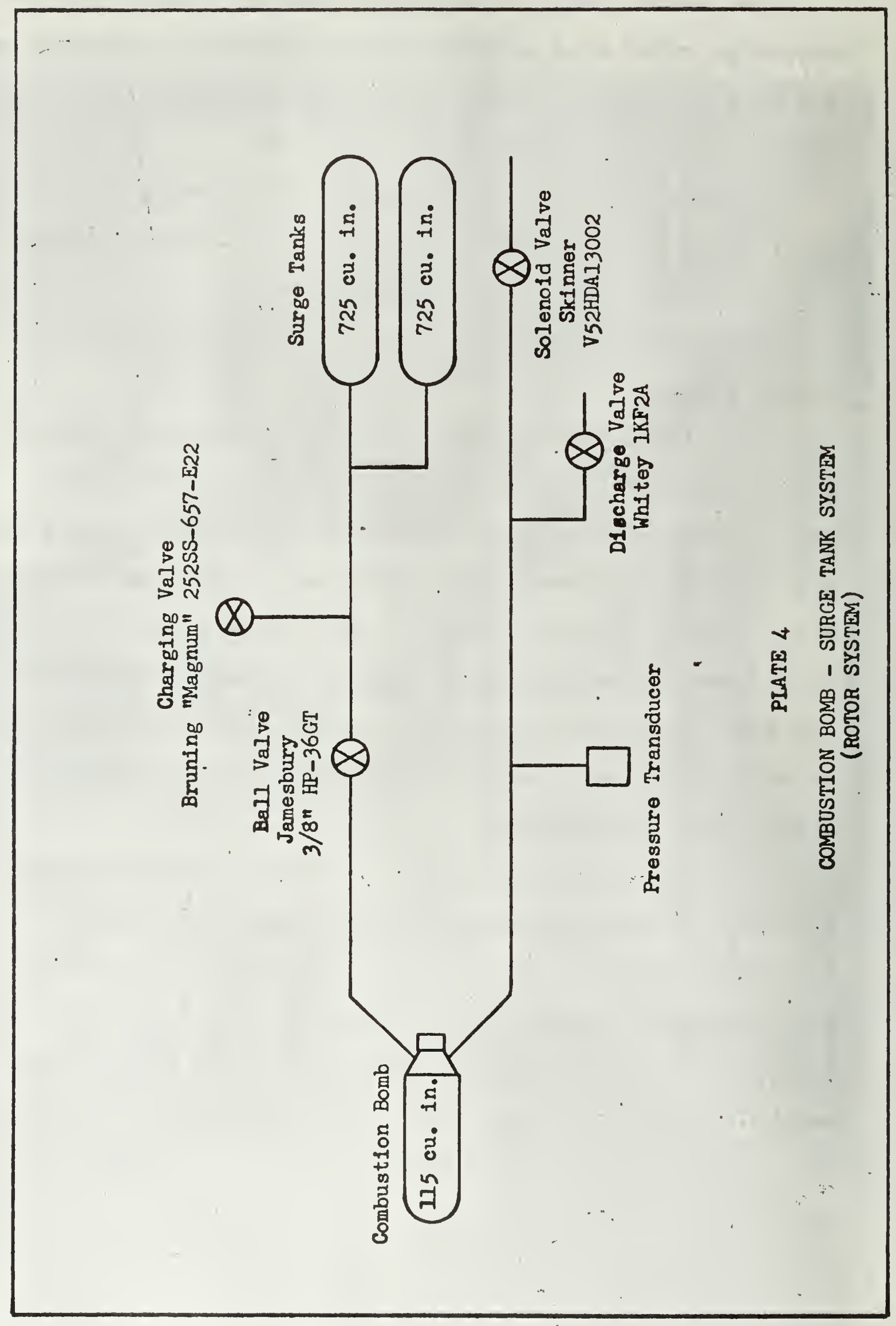




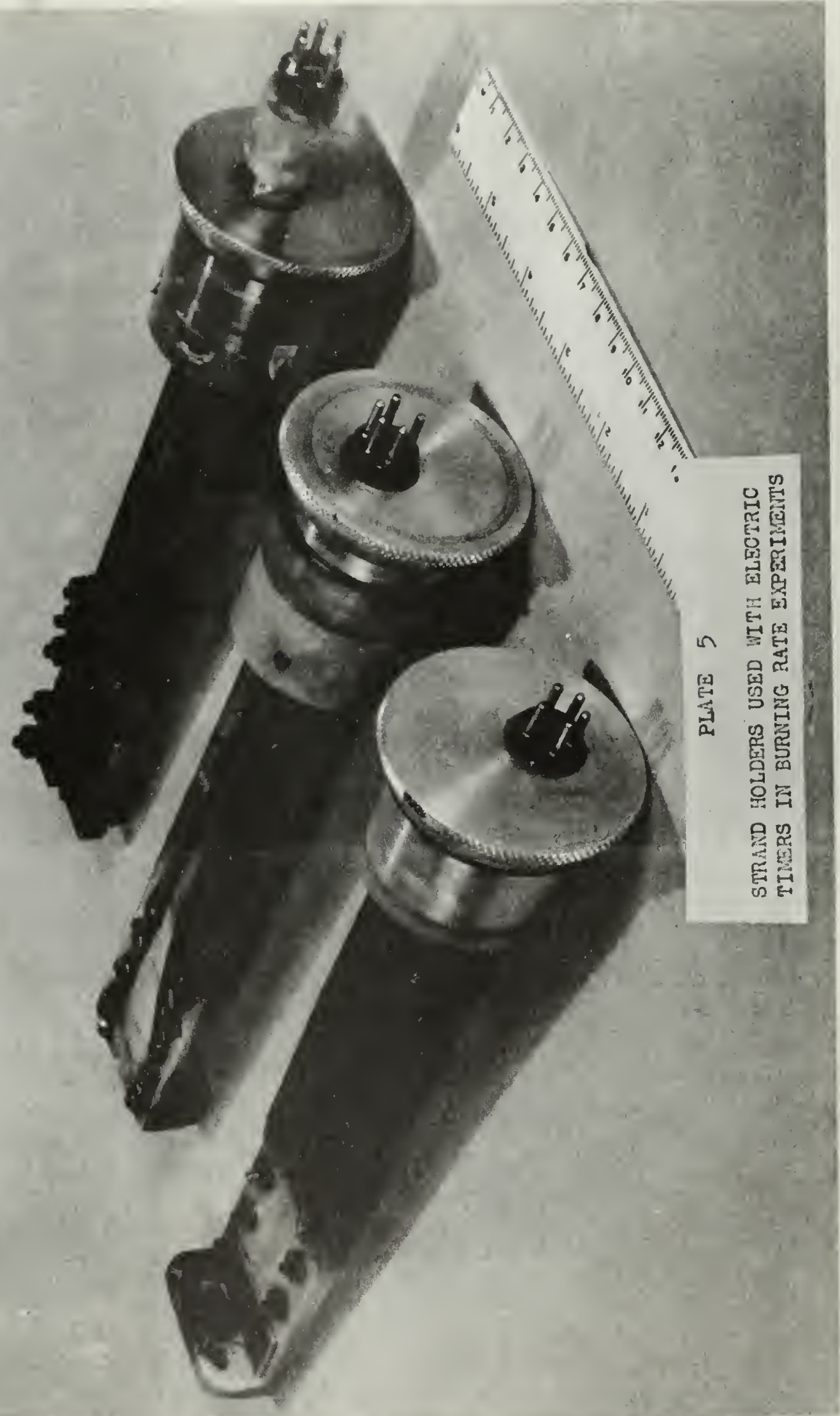




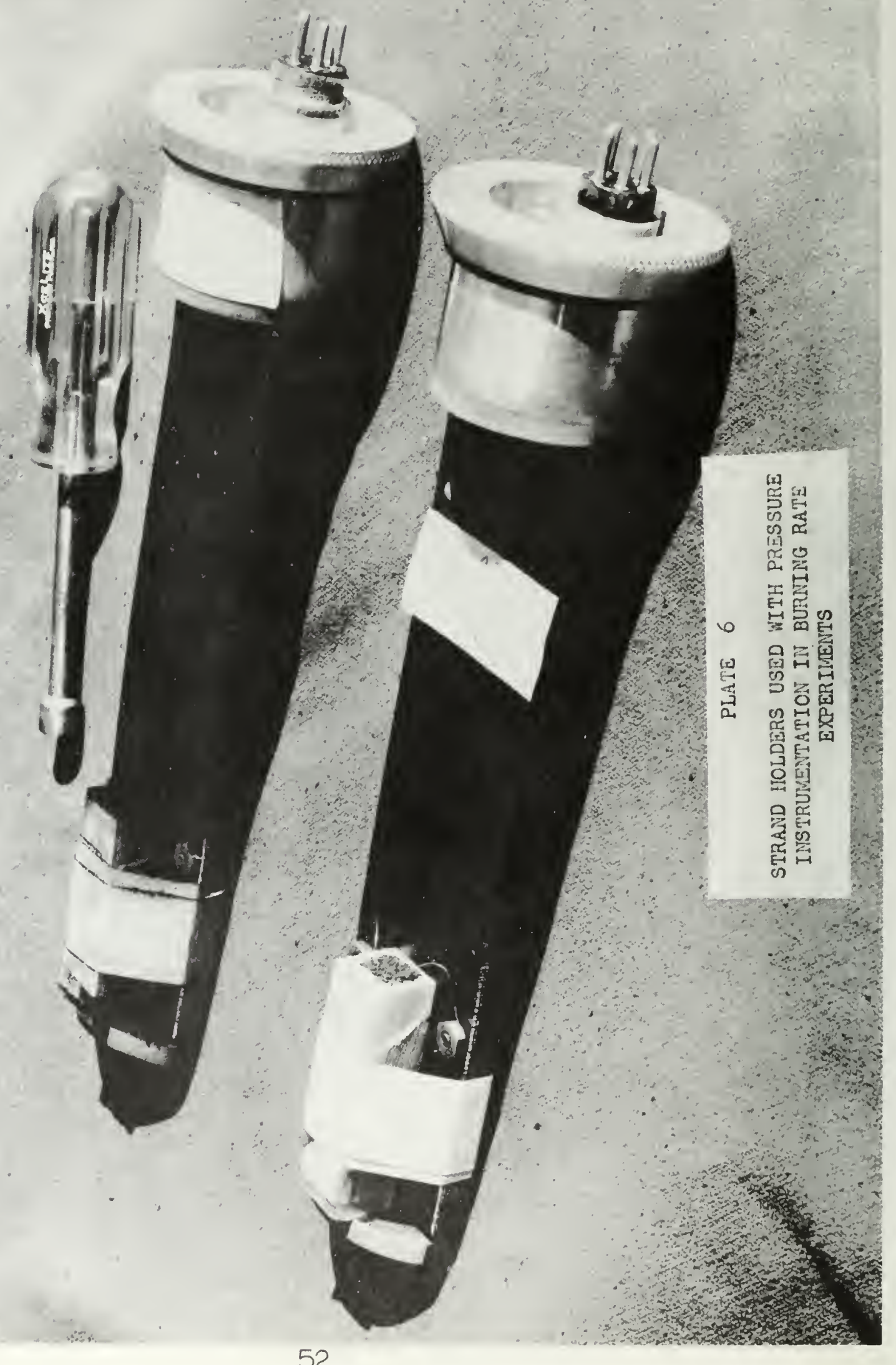




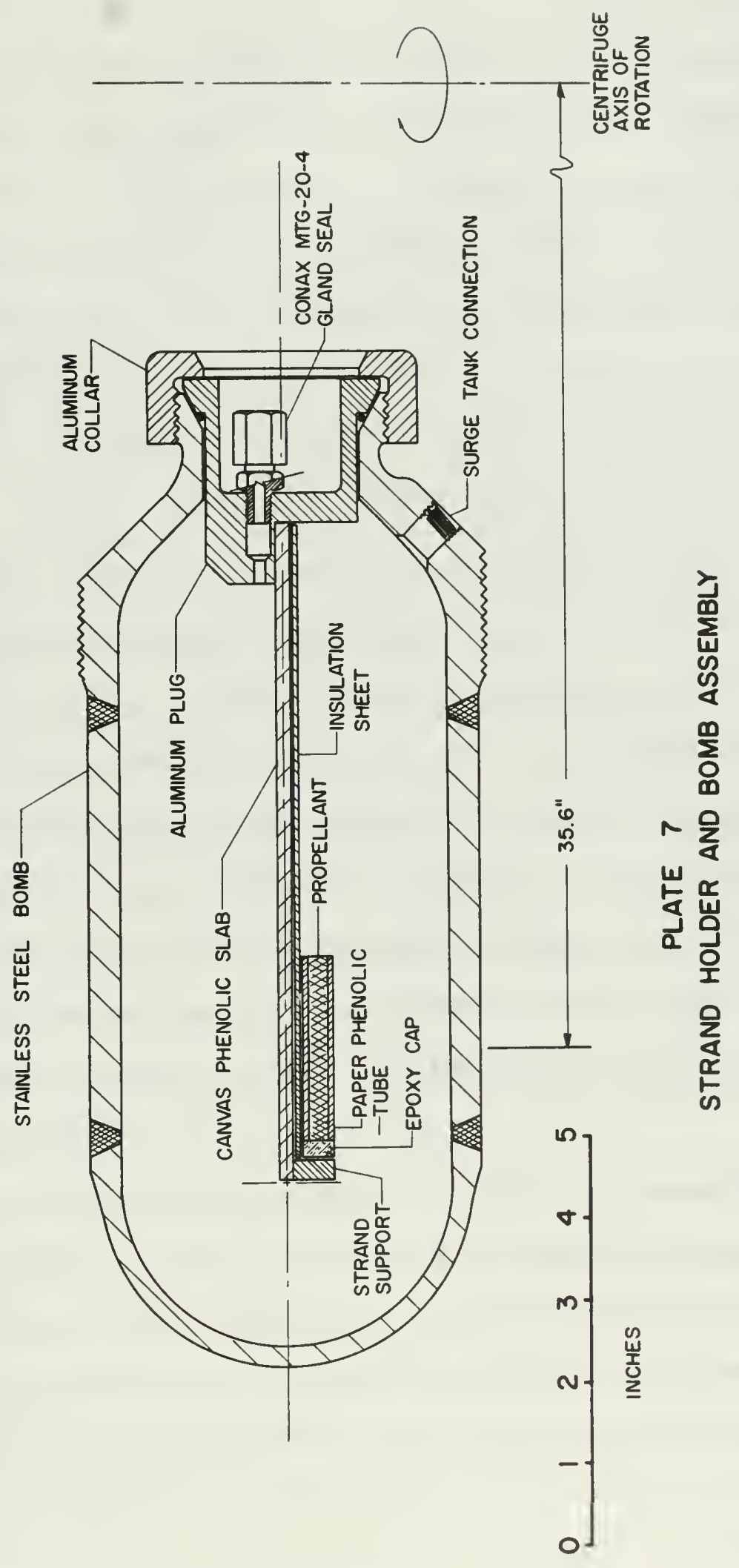

53 
of the slab with APCO epoxy to protect them from contact with the combustion products.

Strand holders for use with the electric timers had five pairs of terminals on the slab; four for the timing circuits and one for the ignition circuit. One terminal of each pair was a common ground to the aluminum plug. The remaining five leads were connected to the Amphenol plug through a single $1 / 8$ in. dia. gland in the aluminum plug. The gland was reamed on the inside with a $10^{\circ}$ tapered reamer so that the inside of the hole looked like a long thin funnel. With the electrical leads installed, the remaining space was filled with APCO epoxy using a hypodermic syringe and needle. The epoxy provided an effective gland seal for internal pressures up to $1000 \mathrm{psig.} \mathrm{At} 1500 \mathrm{psig}$ some leakage occurred. The essential difference between the strand holders designed for use with the electric timers and those designed for use only with the pressure instrumentation was that the latter had only one pair of terminals. Also, commercial gland seals were used exclusively in the two wire strand holders.

The canvas phenolic slab was protected from direct contact with the exhaust flame by a $3 / 32$ in. thick phenolic plastic insulation sheet (FSN 9330282 5641). The insulation sheet covered the entire slab and was held in place by the ignition wire terminal nuts and a \#3-48 screw near the aluminum plug. 
Propellant samples were placed on the strand holder with their bases resting on the strand support. Masking tape wrapped around the sample and strand holder as shown in Plate 6 held the sample securely in place at all acceleration levels up to $2000 \mathrm{G}$.

A drawing of the propellant sample and strand holder installed in the combustion bomb is shown in Plate 7. Instrumentation and Electrical

RPM instrumentation consisted of a SPACO type PA-1 Magnetic Pickup and a Berkeley Model 5545 EPUT Meter. The timing disc had 30 teeth and was mounted behind the shear coupling. See Plate 8 . With a counting period of one second, RPM/2 was read on the EPUT Meter.

A Lebow Model 6109-12 instrumentation slip ring assembly was mounted on the centrifuge shaft above the brake disc. The assembly was rated for 2000 RPM maximum and had coin-silver rings and silver-graphite brushes for low nolse. Electrical leads from the ring terminals passed through two holes in the hollow shaft and connected to the underside of a circular terminal block in the top of the shaft. The slip ring assembly is shown in Plate 9.

Pressure in the combustion bomb was sensed by a DaystromWiancko Type P2-3086 or P2-1251 variable reluctance pressure transducer. The transducer was mounted at the centrifuge axis of rotation in order to minimize the effects of acceleration on the instrument. $28 \mathrm{v} \cdot$ d.c. excitation was provided by a Hewlett-Packard Model $721 \mathrm{~A}$ power supply. The zero to $5 \mathrm{v}$. d.c. output was read on a Weston Model 911 D.C. Voltmeter having an impedance of $20 \mathrm{~K} \Omega$ /volt. 


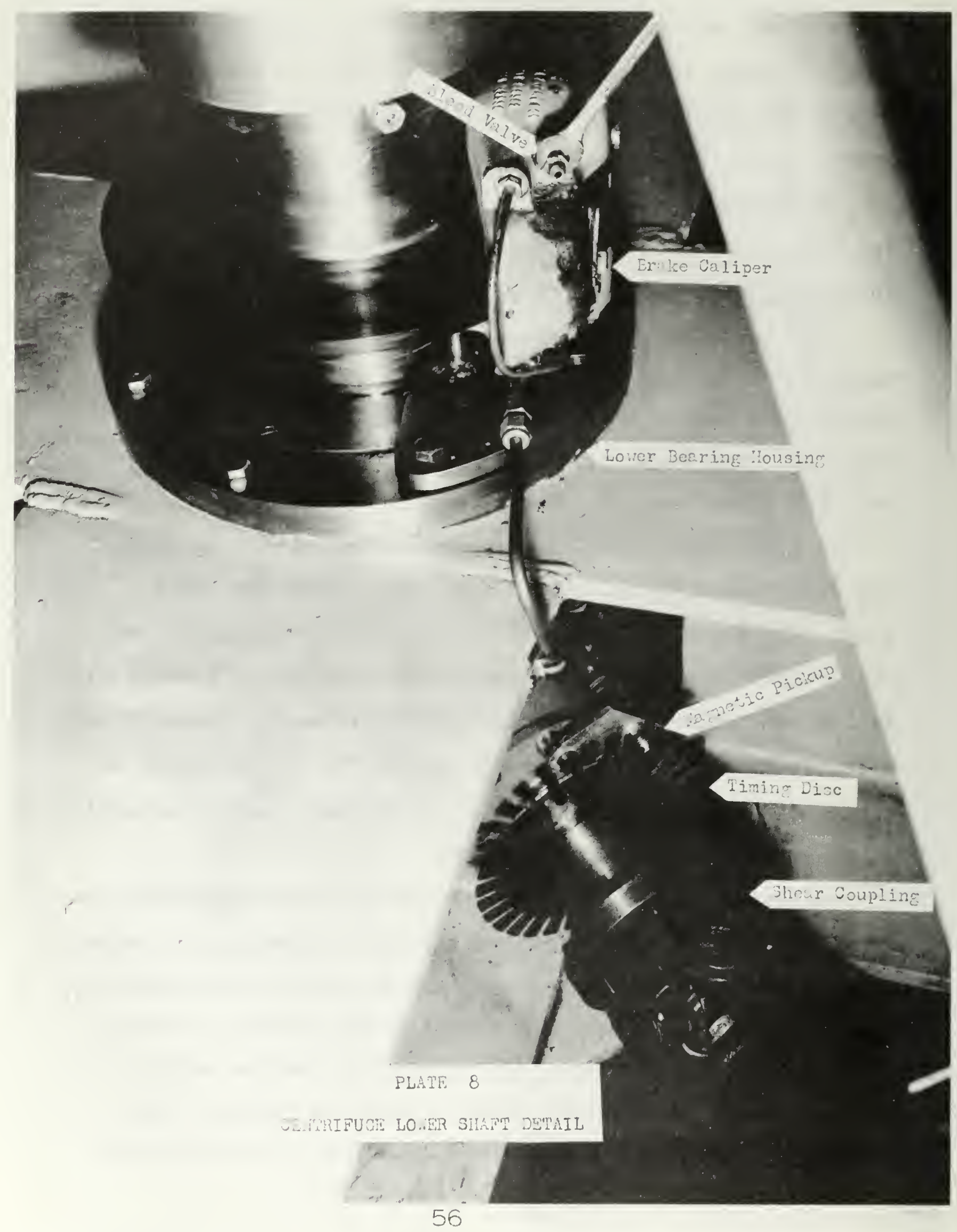


Pressure change in the bomb was recorded on a Honeywell Model 1508 Visicorder. A bucking voltage was applied to the transducer output with a 6 volt dryeell battery and a potentiometer. The resultant small signal then went to a Kintel Model 112A single ended D.C. Amplifier. Amplifier output was fed to an M400-120 galvanometer in the Visicorder. Variable series and shunt resistors in the galvanometer circuit permitted adjustment of chart span and galvanometer damping. Zero adjustment was provided by changing the bucking voltage potentiometer setting. A schematic drawing of the circuit is shown in Plate 10 .

Average burning rate was determined by recording elapsed time during burning over a known distance. The primary means utilized the pressure time history recorded on the Visicorder chart. Burning was assumed to commence when the pressure began to rise, and end when the pressure stopped rising. Dividing the initial sample length by the total burning time gave the average burning rate.

The secondary means utilized timing wires and electric timers. Three Standard Electric Time Co. Model S-1 Timers were used for recording elapsed time. $115 \mathrm{v}$. a.c. power to the timer motor clutch coils was controlled by Magnecraft W133MPCX-3 mercury-wetted contact SPDT relays.

Four timing wires were equispaced in the propellant sample. The timing wires were 0.008 inch diameter Pyro-fuse, which was composed of an aluminum core sheathed in palladium. 


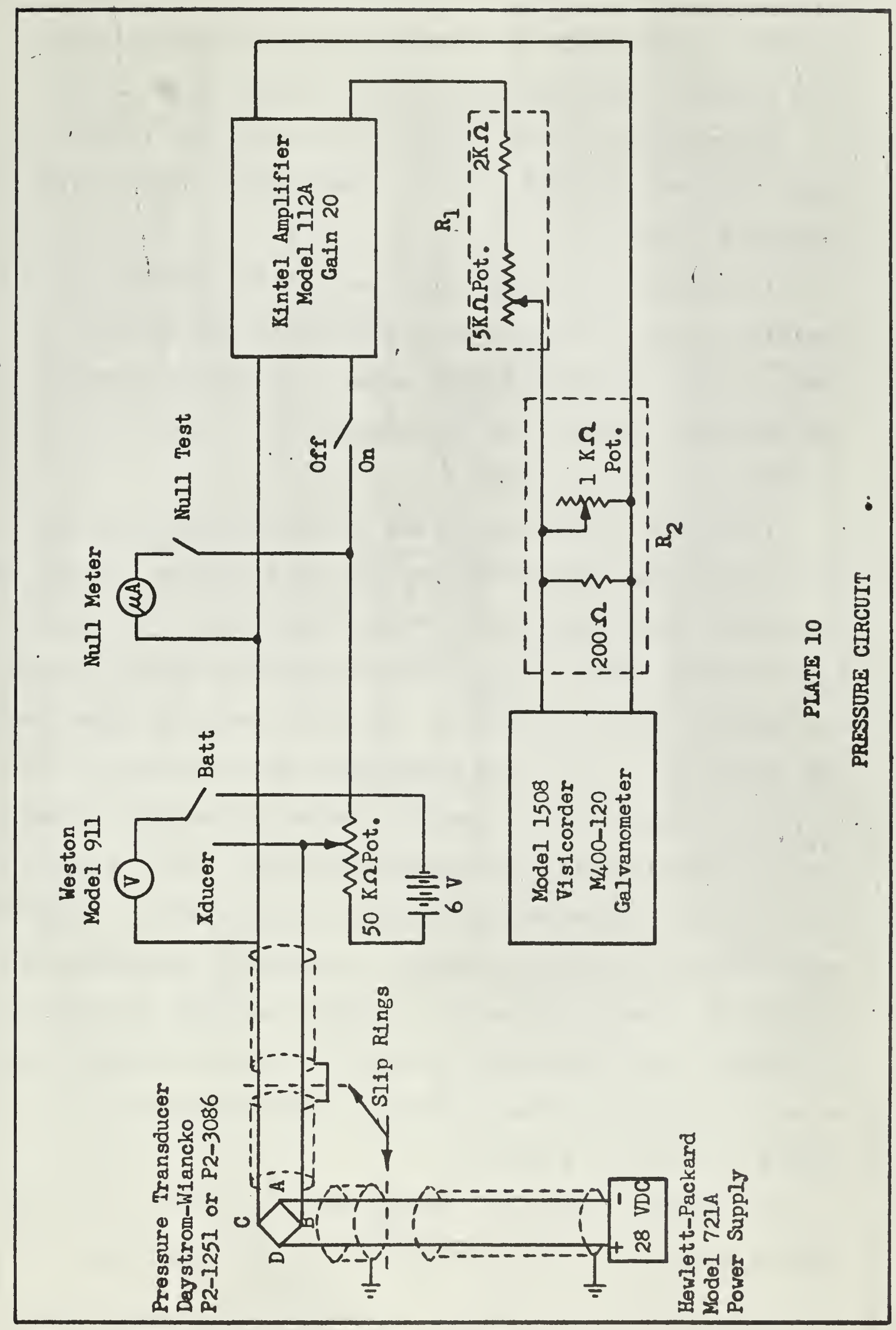


At $600^{\circ} \mathrm{F}$, the metals alloyed rapidly and exothermically, thus breaking the $6 \mathrm{v}$. timer relay holding coil circuits. The propellant sample was ignited by a \#32 (0.008 in. dia.) nichrome resistance wire. Power was supplied from the 12 v. d.c. bus.

Continuity of the ignition and timing circuits could be checked by means of a rotary switch which applied $6 \mathrm{v}$. d.c. power to five continuity test lamps. Schematic drawings of the ignition, timing, and continuity test circuits are shown in Plates 11, 12, 13, and 14 .

Vibration of the centrifuge structure was monitored from the control station by reading on a Honeywell Model 1508 Visicorder the output from a vibration pickup. The pickup was a Statham Model A5A-15-350 accelerometer ( $\pm 15 \mathrm{~g})$ which was mounted on the centrifuge structure near the upper bearing (Plate 3). $10 \mathrm{v} \cdot \mathrm{d} \cdot \mathrm{c}$. excitation was provided by the 28 v. d.c. output from a Hewlett-Packard Model $721 \mathrm{~A}$ power supply in series with a dropping resistor. The $\pm 4 \mathrm{mv}$. output from the accelerometer was fed directly into an M200-120 galvanometer in the Visicorder. A schematic drawing of the circuit is shown in Plate 15. A microphone was located just below the lower bearing at the base of the centrifuge. Audio noise thus picked up was passed through an amplifier to earphones worn by the operator.

The engine electrical system was a standard $6 \mathrm{v}$. automobile system. A schematic drawing is shown in Plate 16. 


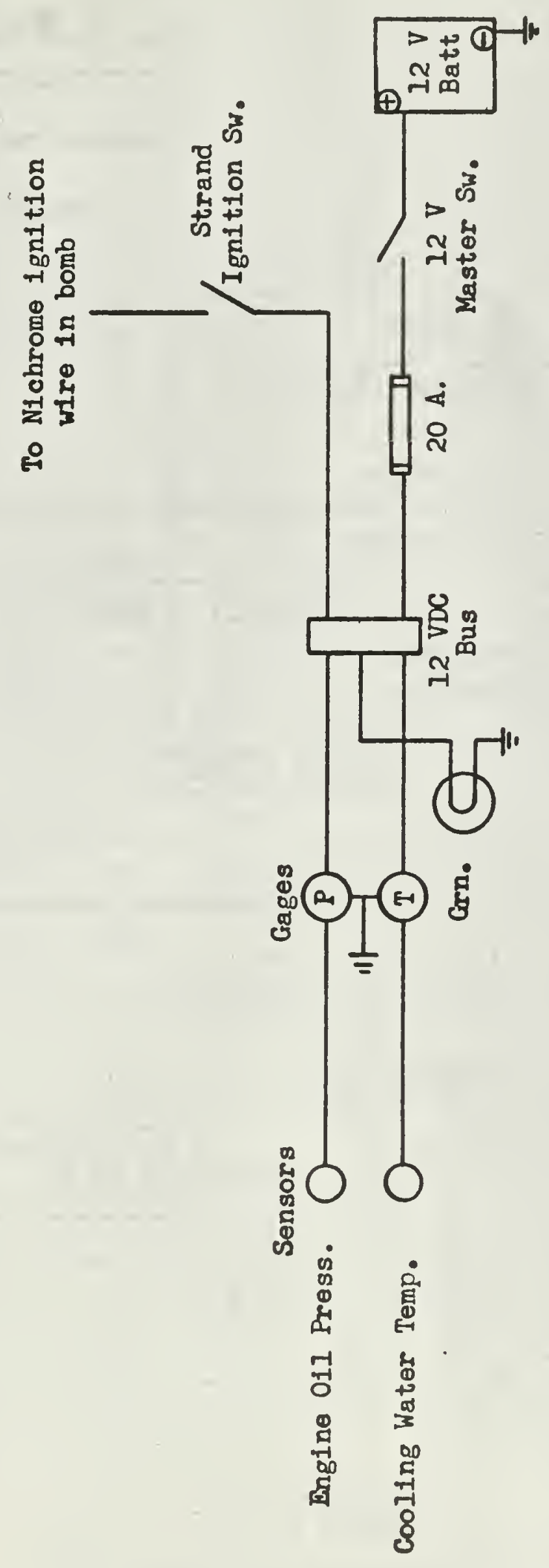

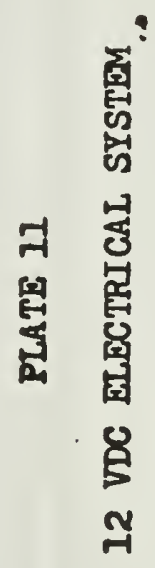




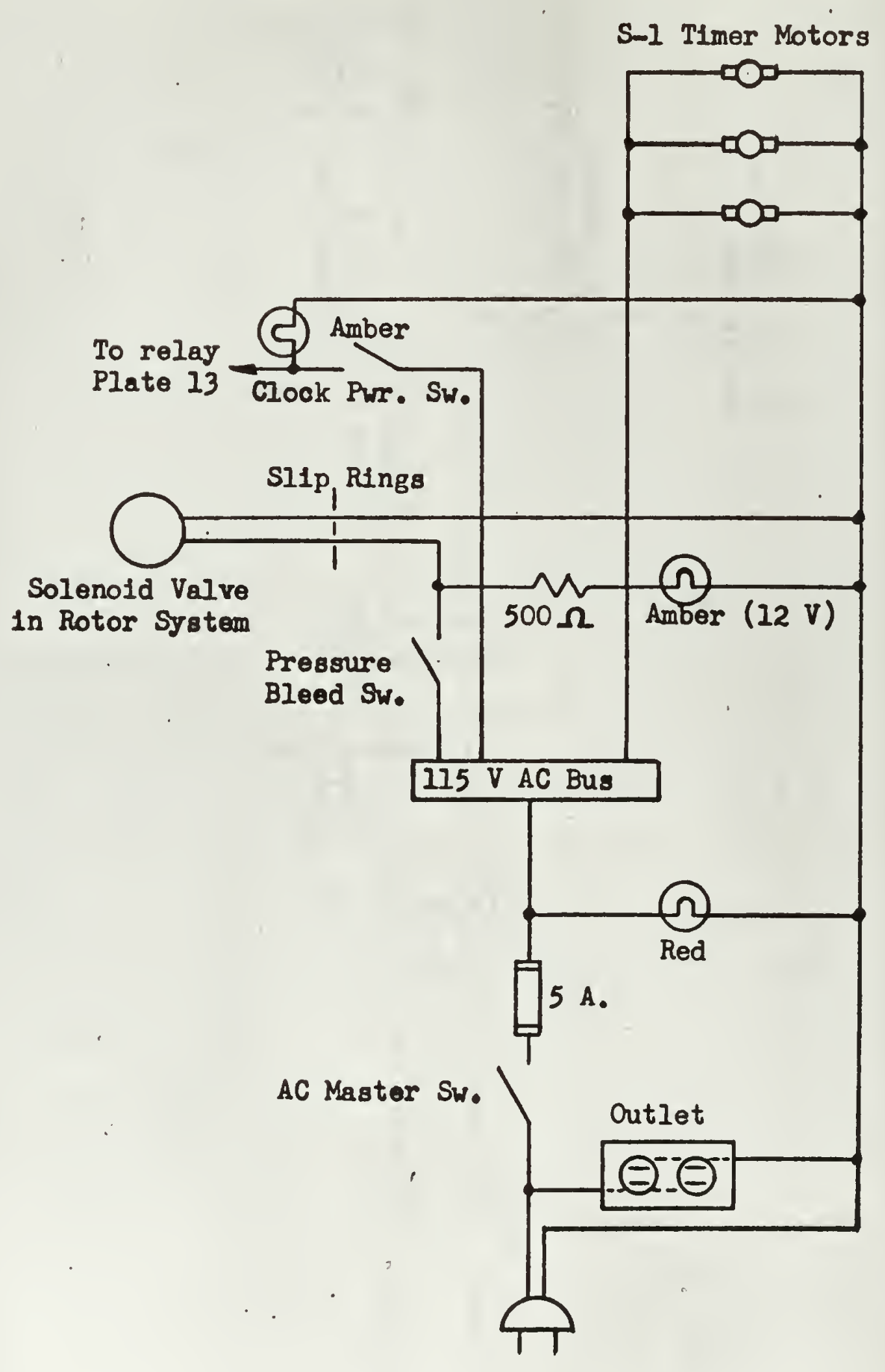

PLATE 12

115 V AC ELECTRICAL CIRCUIT 


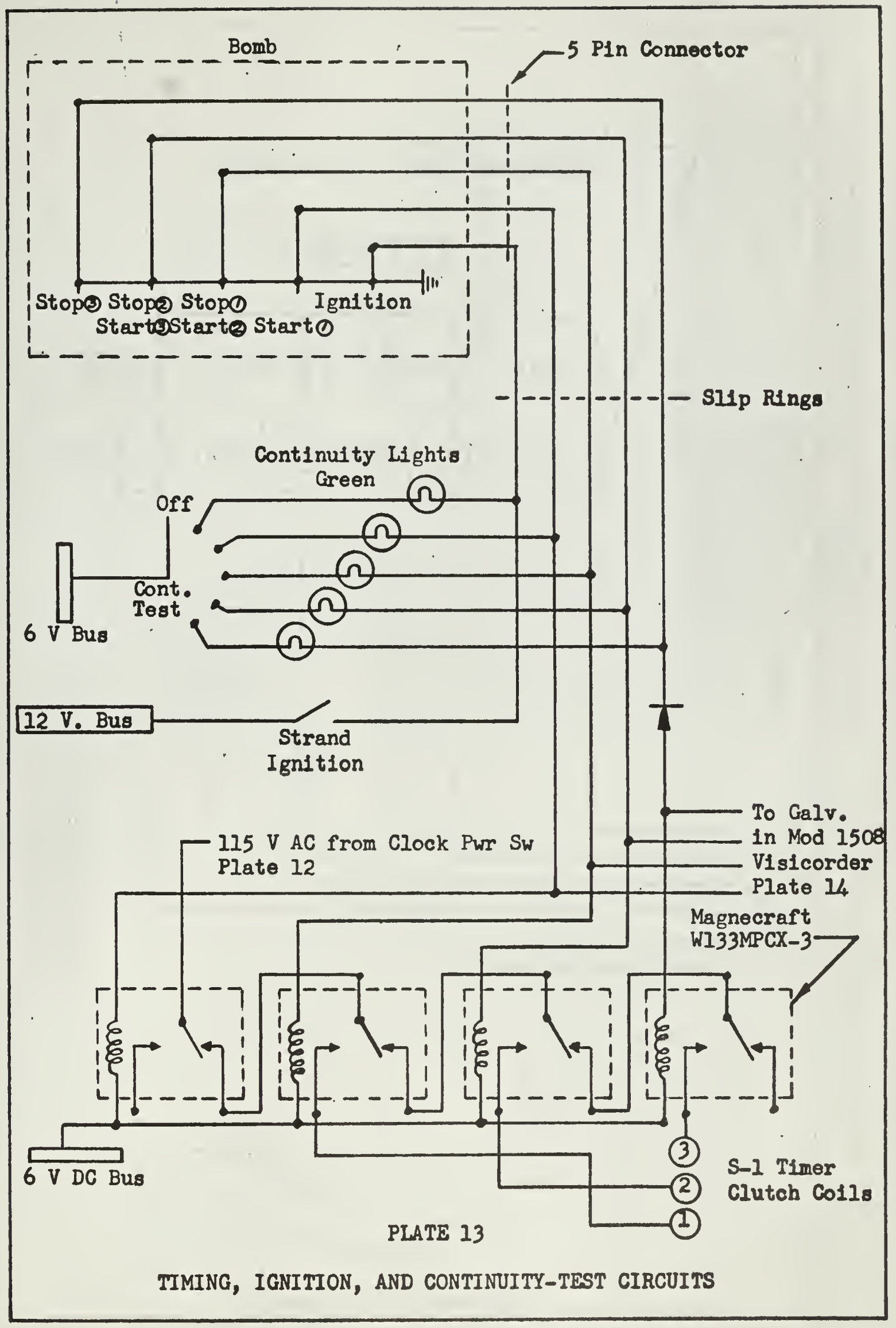




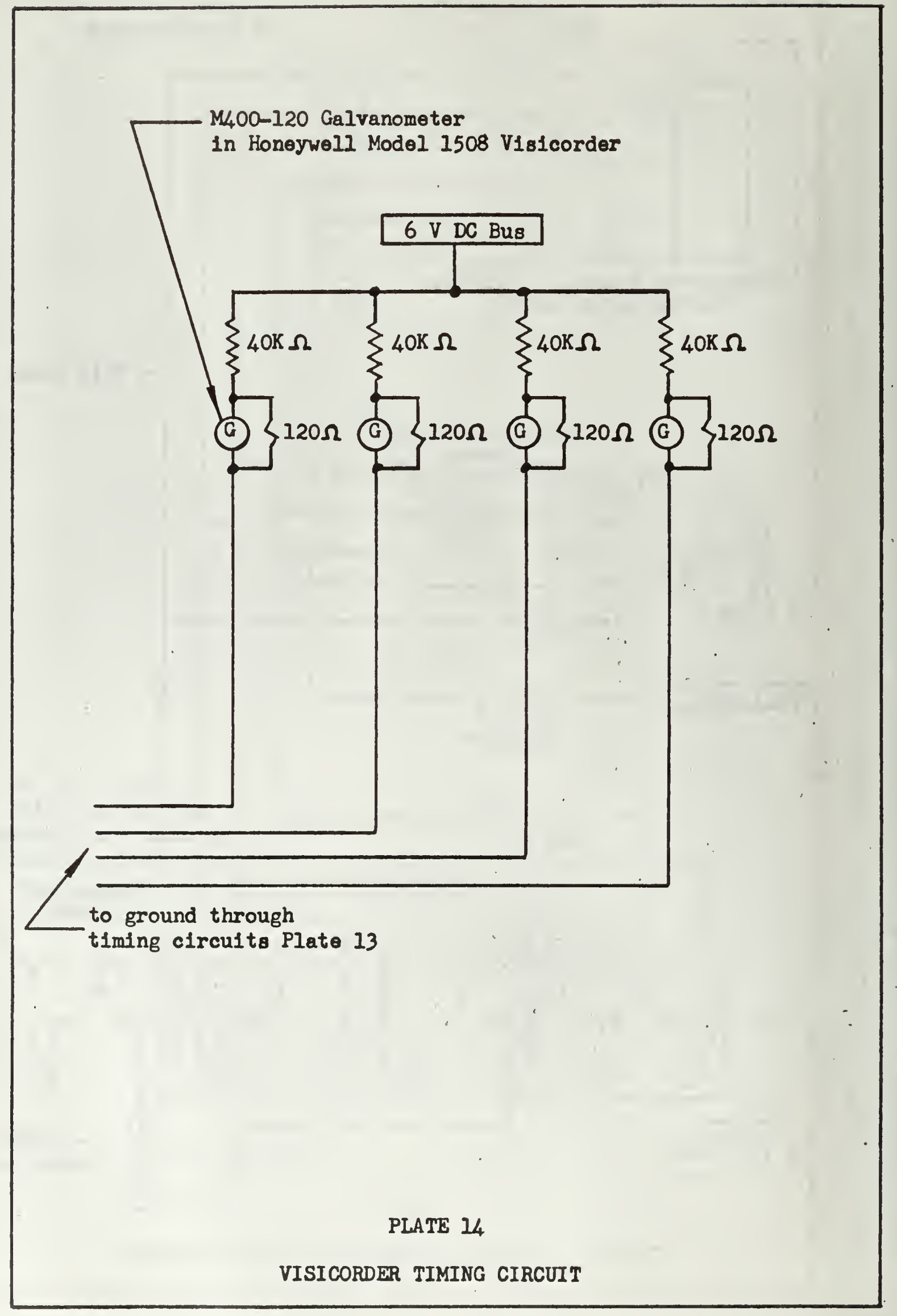




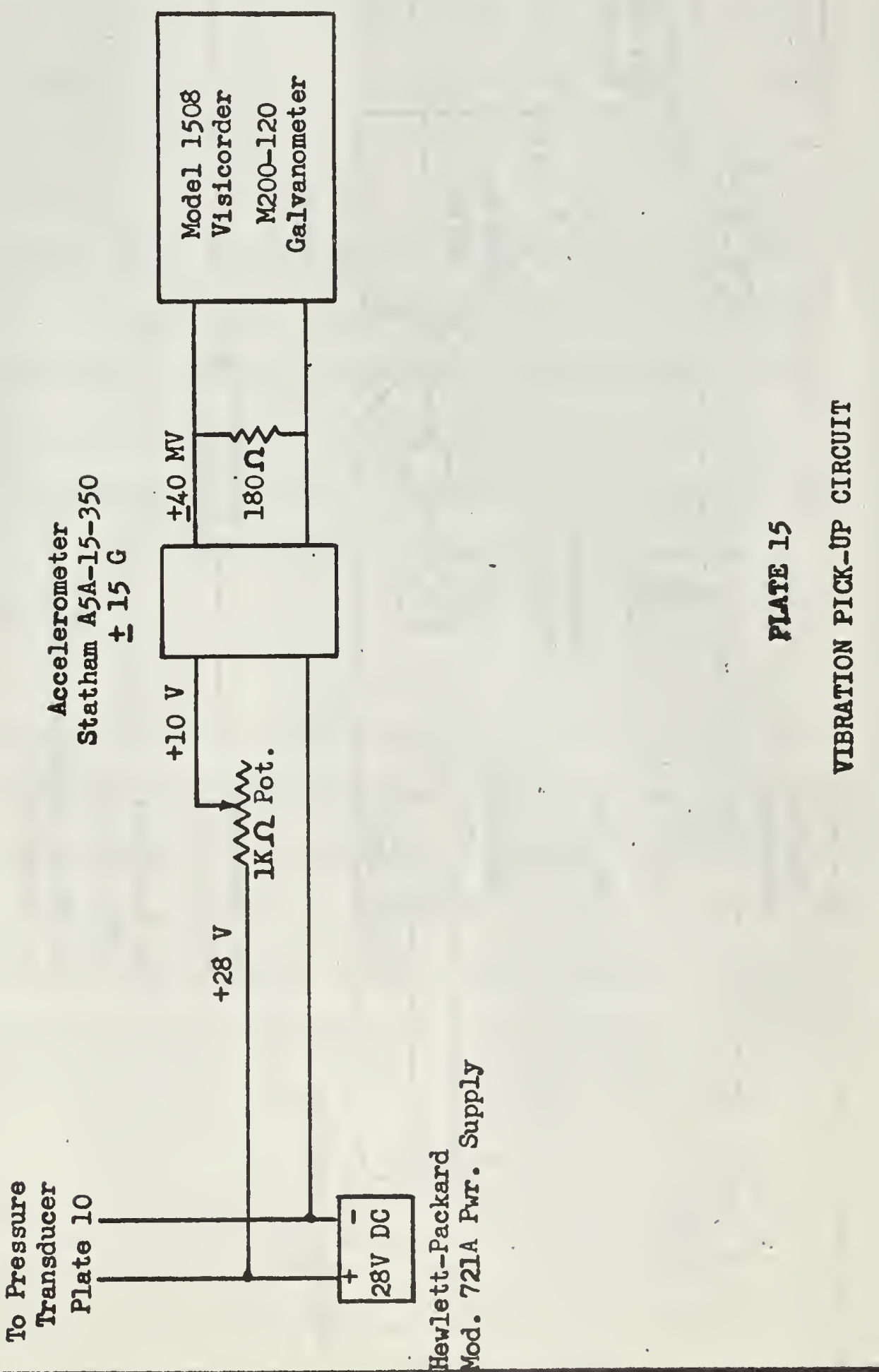




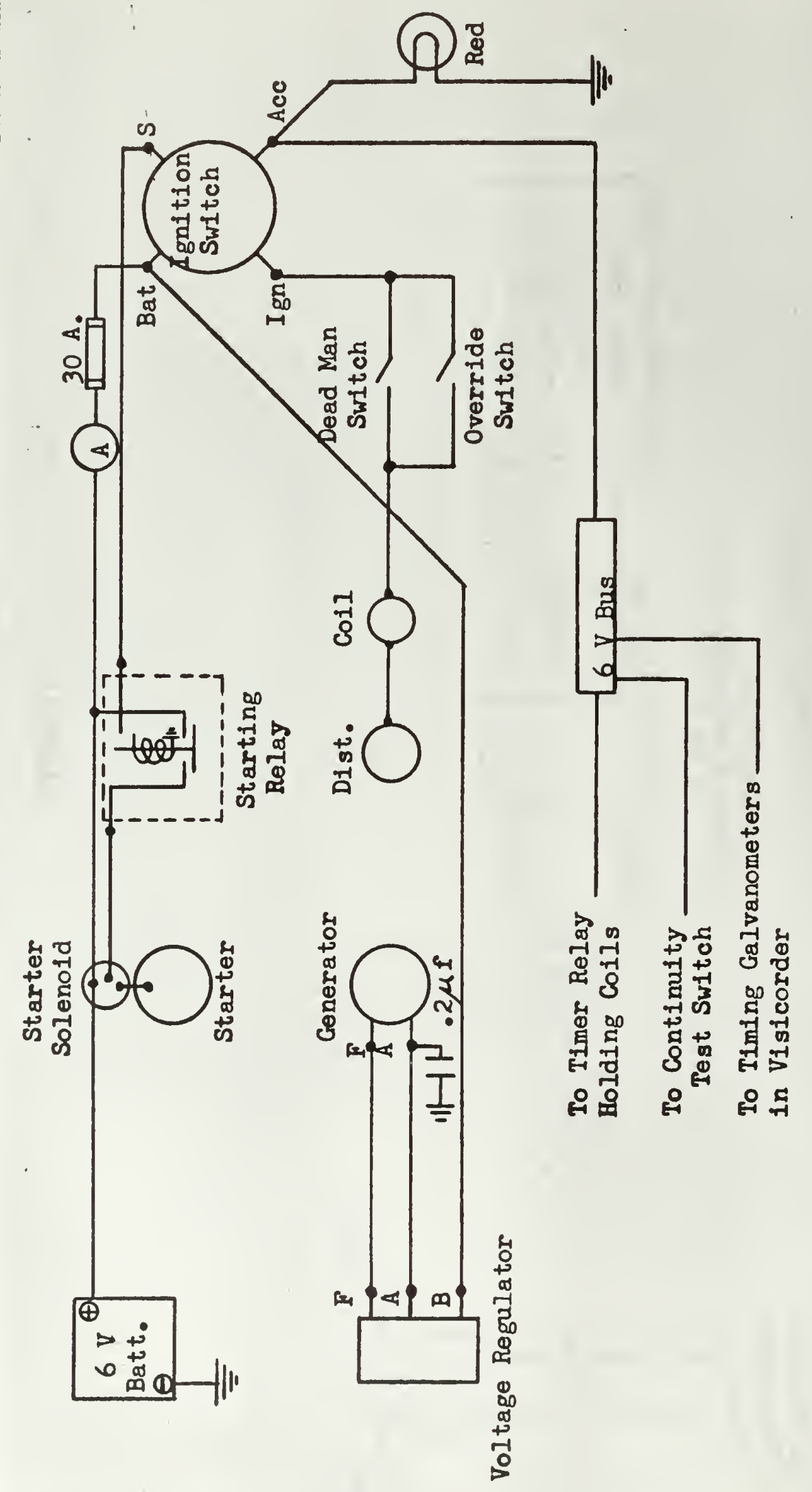

$r$ 


\section{Nitrogen Charging System}

The nitrogen charging system consisted of a fourbottle manifold, regulator, gauge panel and flexible charging hose. The manifold was a Victor "Simplex" discharging type with a shut-off valve for each bottle and a master shut-off valve at the manifold outlet. The pressure regulator was a Victor GD 31 Gas-o-dome regulator, rated for 3600 psi inlet and outlet pressure.

On the gauge panel were mounted three Marsh Type 220-3S pressure gauges with pressure ranges of $0-1000$ psig, 0-3000 psig, and 0-5000 psig. Suitable valves permitted the selection of the appropriate gauge for use in pressurizing the surge tanks and combustion bomb. The flexible charging hose was connected to the rotor system by means of a quickdisconnect coupler. Drive System

The centrifuge was driven by a 1954 Chevrolet engine with a 1950 Powerglide transmission. Power was transmitted to the centrifuge via an automobile drive shaft and a Boston VR158 Spiral Miter Gear Box. A $285 \mathrm{ft}$-1b shear coupling was fitted on the miter gear input shaft, and a double strand roller chain flex-coupling transmitted the torque from the miter gear vertical output shaft to the centrifuge shaft. Controls

The engine throttle setting was controlled by an Adel ISOdraulic Remote Control System. The centrifuge was equipped with a hydraulically actuated disc-type brake. 
The brake was actuated by a foot pedal, and a hand operated cam was used to lock the brake in the ON position. The brake components are shown in Plates 2 and 8 .

A deadman switch was incorporated in the engine ignition circuit as a safety precaution.

\section{PROPELLANT SPECIMENS}

\section{Formulations}

Three basic composite propellants were utilized in this investigation. The first with a polyurethane binder, the second with a carboxy-terminated polybutadiene binder, and the third with a PBAN binder. All three propellants contained ammonium perchlorate oxidizer (AP), and various amounts of spheriodal aluminum powder (AI).

The polyurethane and carboxy-terminated polybutadiene propellants were prepared by the U.S. Naval Ordnance Test Station (NOTS), China Lake, California. The PBAN propellants were prepared by United Technology Center (UTC), Division of United Aircraft Corp., Sunnyvale, California.

To facilitate discussion in subsequent sections, the polyurethane propellants have been designated the X100 series. The carboxy-terminated polybutadiene propellant is the $\mathrm{X} 200$, and the PBAN propellants are the $\mathrm{X} 300$ series. Specific formulations are as follows: 
X100 series. Three variations in the $\mathrm{X} 100$ series were investigated.

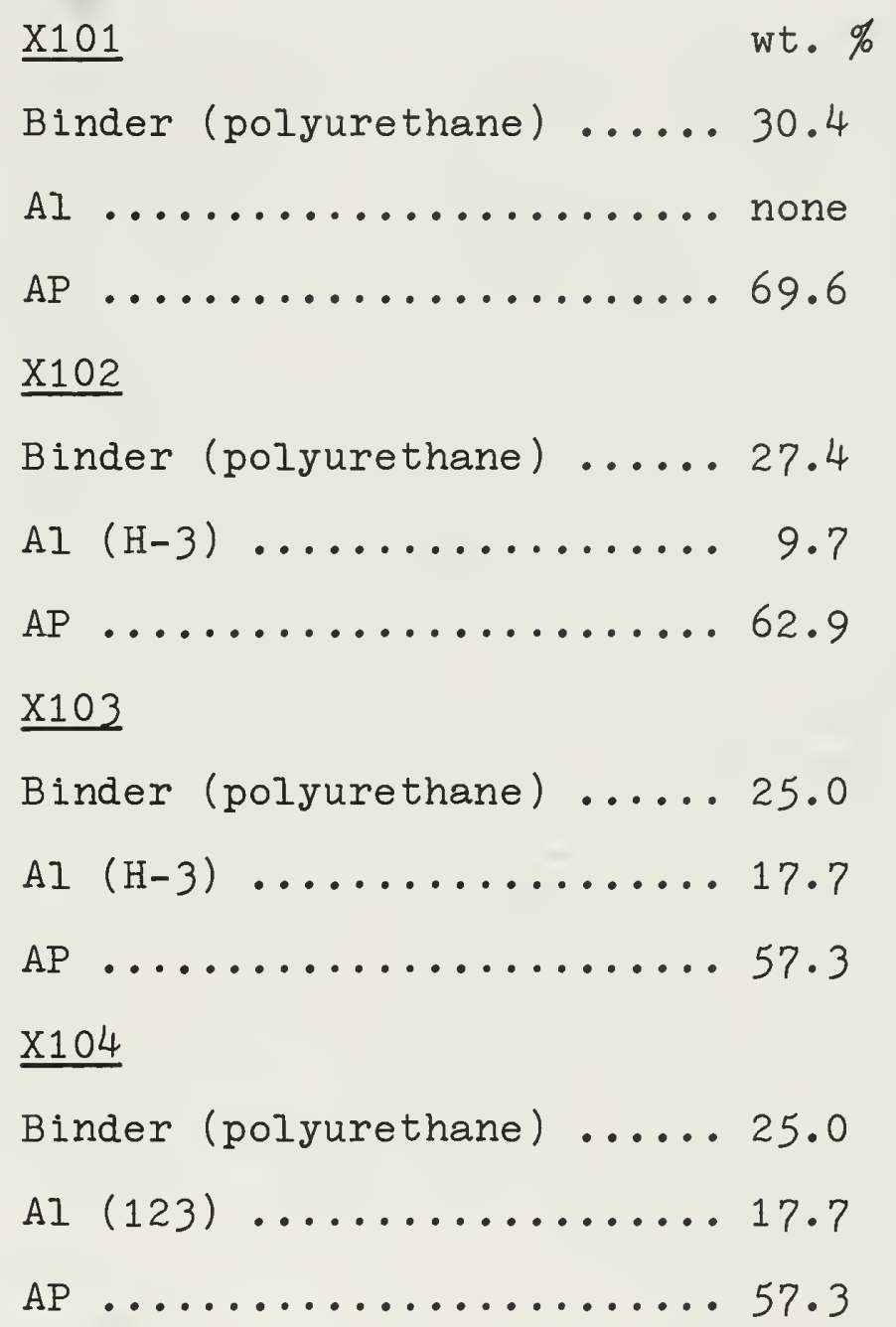

The AP mass median diameter was 195 microns (Tyler sieve). The particle size distribution is shown in Figure 6. The H-3 and Al(123) aluminum powders had mass median diameters of 6.3, and 31 microns respectively (micromerograph). The particle size distributions are shown in Figure 7.

\section{X200 propellant. wt. \%}

Binder (carboxy-terminated polybutadiene) ..... 14.0

AI $(\mathrm{H}-5) \ldots \ldots \ldots \ldots \ldots \ldots . \ldots 17.0$

AP (tri-modal) ........6 69.0 


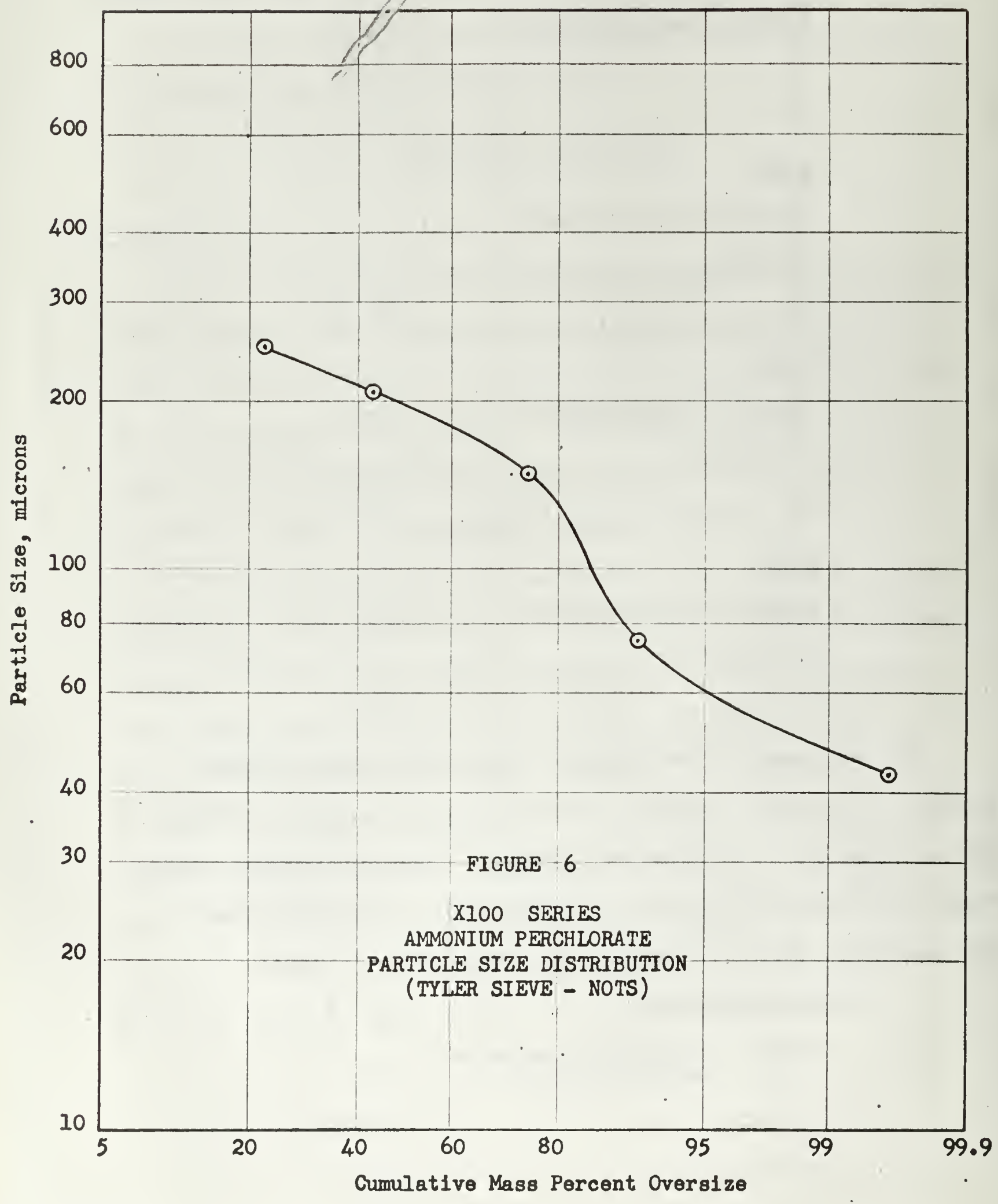




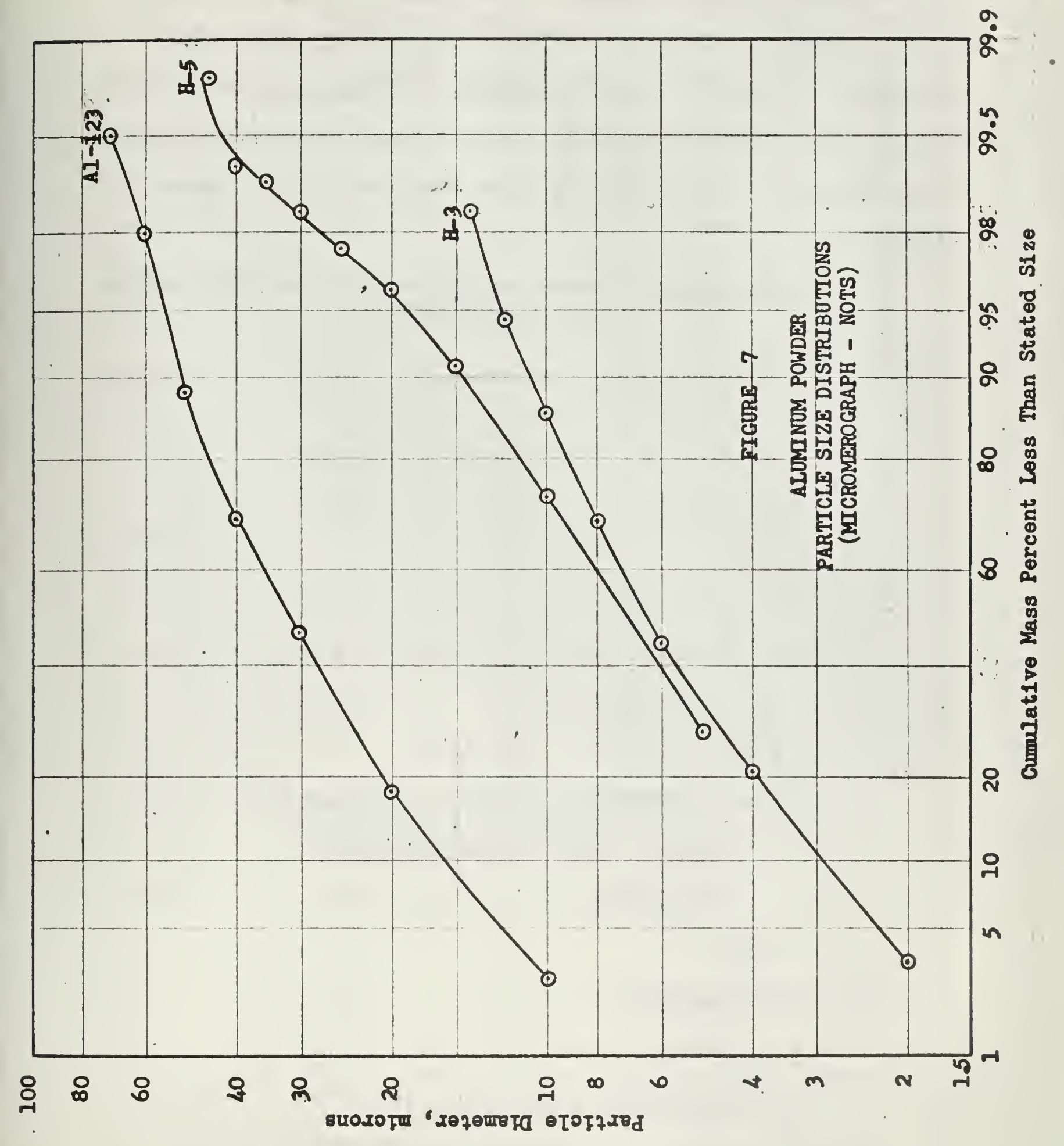


The H-5 aluminum powder had a mass median diameter of 7.1 microns (micromerograph). The particle size distribution is shown in Figure 7. The tri-modal AP consisted generally of $20 \% 600$ micron spheres, $50 \%$ as received, and 25\% ground. The mass median diameter was 195 microns (micromerograph). The particle size distribution is shown in Figure 8.

X300 series. Three variations in the $X 300$ series were investigated.

X301

wt. $\%$

Binder (PBAN) $\ldots \ldots \ldots \ldots 19.5$

Al ...................nne no

AP (bi-modal) $\ldots \ldots \ldots \ldots .60 .5$

$\underline{\mathrm{X} 302}$

Binder (PBAN) $\ldots \ldots \ldots \ldots 18.8$

AI $(H-10) \ldots \ldots \ldots \ldots \ldots, 4.0$

AP (bi-modal) $\ldots \ldots \ldots \ldots \ldots 77.2$

$\underline{\mathrm{X} 303}$

Binder (PBAN) $\ldots \ldots \ldots \ldots 16.4$

AI $(H-10), \ldots \ldots \ldots \ldots \ldots \ldots .16 .1$

AP (bi-modal) $\ldots \ldots \ldots \ldots 667.5$

$\underline{\mathrm{X} 304}$

Binder (PBAN) $\ldots \ldots \ldots \ldots 16.2$

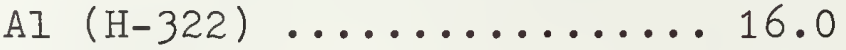

AP (bi-modal) $\ldots \ldots \ldots \ldots .667 .8$

The H-10 aluminum powder had a mass median diameter of approximately 14 microns, and the H-322 aluminum powder had a mass median diameter of 47 microns (micromerograph). 


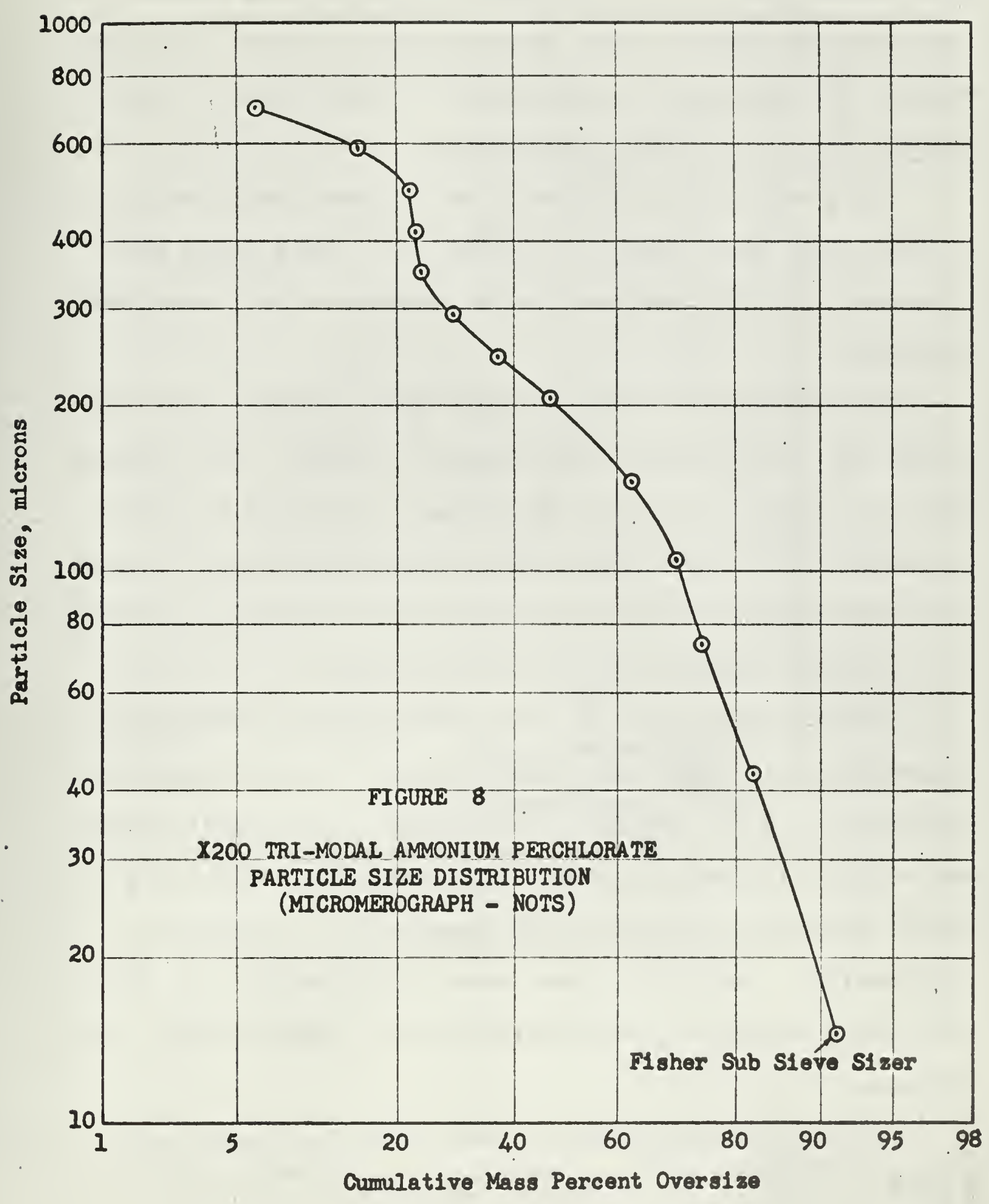


The bi-modal AP consisted of $2 / 3$ as received (190 micron mass median dia.) and $1 / 3$ ground ( 9 micron mass median dia.). The aluminum particle size distribution is shown in Figure 9, and the AP particle size distributions are shown in Table I. Preparation of Propellant Specimens

The propellants obtained from UTC were received cast in 10 inch long paper phenolic tubes. The tubes had a wall thickness of $1 / 16$ inch and inside diameters of $\frac{1}{4}$ inch and $3 / 8$ inch.

The propellants obtained from NOTS had been cast in blocks and then machined into square strands. The strands were 0.2 inch $x 0.2$ inch $x 4 \frac{1}{2}$ inches long and 0.4 inch $x$ 0.4 inch $\times 4 \frac{1}{2}$ inches long. Each strand was spirally wound with three turns of heavy-duty household string and placed in a form for application of the inhibitor.

The inhibitor used on the strands was an unsaturated polyester resin cured with a peroxide. The resin used was Selectron 5119 (Pittsburgh Plate Glass). The curing agent was a $60 \%$ solution of methyl ethyl ketone peroxide in dimethyl phthalate ("Garox," Ram Chemical Co., Gardena, California). The ratios used were $92 \%$ selectron and $8 \%$ of the curing catalyst. The inhibitor was cured at room temperature.

The outside dimensions of the inhibitor cases were $\frac{1}{2}$ inch $x \frac{1}{2}$ inch $x 43 / 4$ inches for the $0.2 \times 0.2$ inch strands and $3 / 4$ inch $x 3 / 4$ inch $\times 43 / 4$ inches for the 0.4 


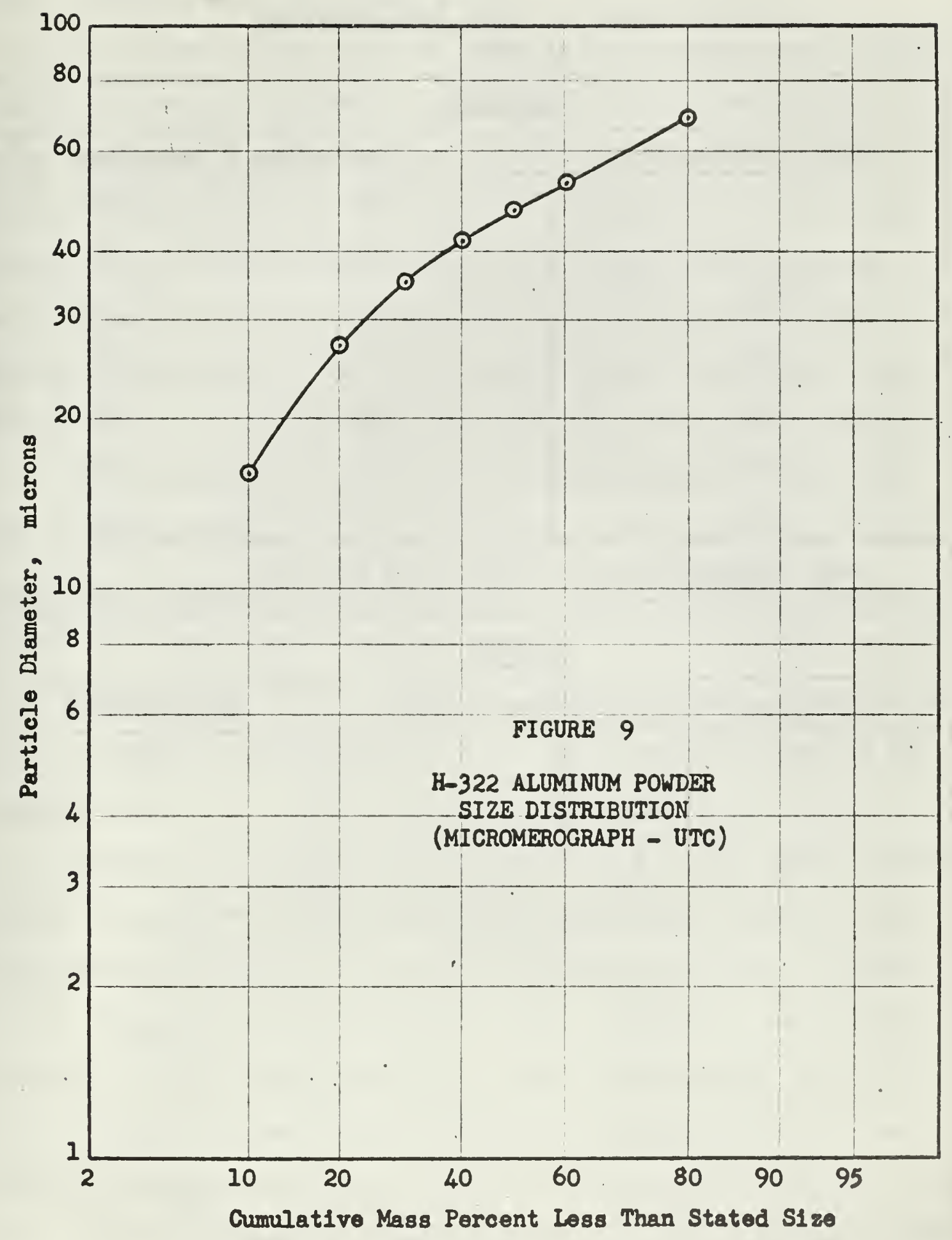


TABLE I

$\underline{\text { X300 SERIES AP SIZE DISTRIBUTION }}$

(UTC)

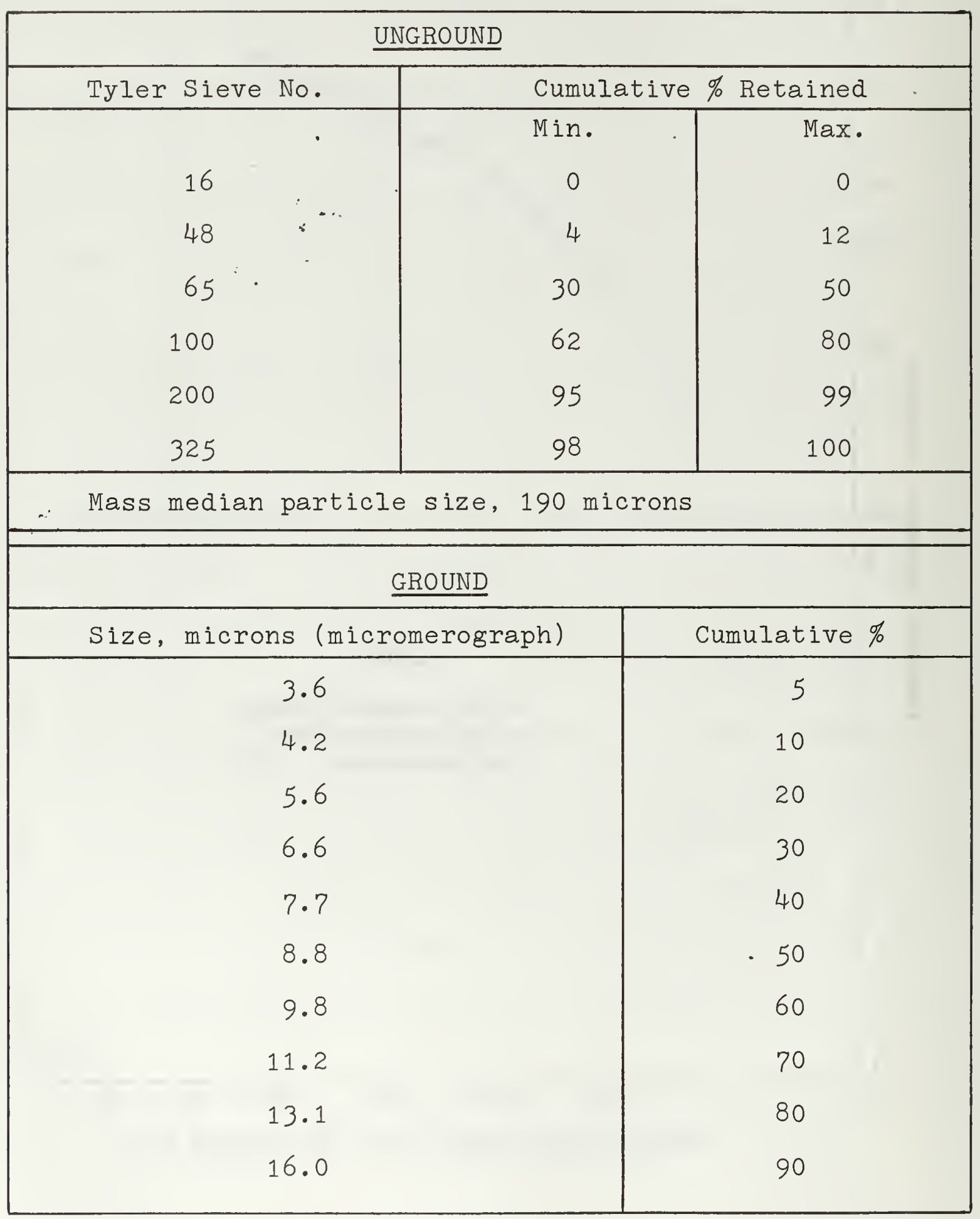


x 0.4 inch strands. The propellants were received from NOTS processed as described above.

All propellants received from UTC and NOTS were $\mathrm{x}$-rayed for voids using a standard overhead medical $x$-ray unit. only about one percent of the $X 100$ series and $X 200$ aluminized propellants were rejected on the basis of the $\mathrm{x}$-rays. Nearly $60 \%$ of the non-aluminized X101 propellant samples were rejected due to many small voids in the propellant. Approximately $30 \%$ of the $\mathrm{X} 300$ series propellants were rejected due to voids between the propellant and the tube wall The propellants selected for investigation were then cut into appropriate lengths. Those to be used with timing wires were cut with a hacksaw using the jigs shown in Plate 17. The sample lengths were $23 / 4 \mathrm{in}$. for $3 / 4 \mathrm{in}$. wire spacing and $2 \frac{1}{4}$ in. for $\frac{1}{2}$ in. wire spacing. Those to be used without timing wires were cut on a bandsaw into $2 \frac{1}{4} \mathrm{in}$. lengths.

Samples to be used with the electric timers were drilled for timing wires with a \#72 (0.025 inch dia.) drill. The jigs shown in Plate 17 were used for hole spacing. Guide holes in the jigs were located with a dividing head within $0.0005 \mathrm{in}$. Four holes were drilled in the sample at $\frac{1}{2}$ inch or $3 / 4$ inch intervals with at least $\frac{1}{4}$ inch between the beginning of the sample and the first timing wire, and at least $\frac{1}{4}$ inch between the last timing wire and the end of the sample. 


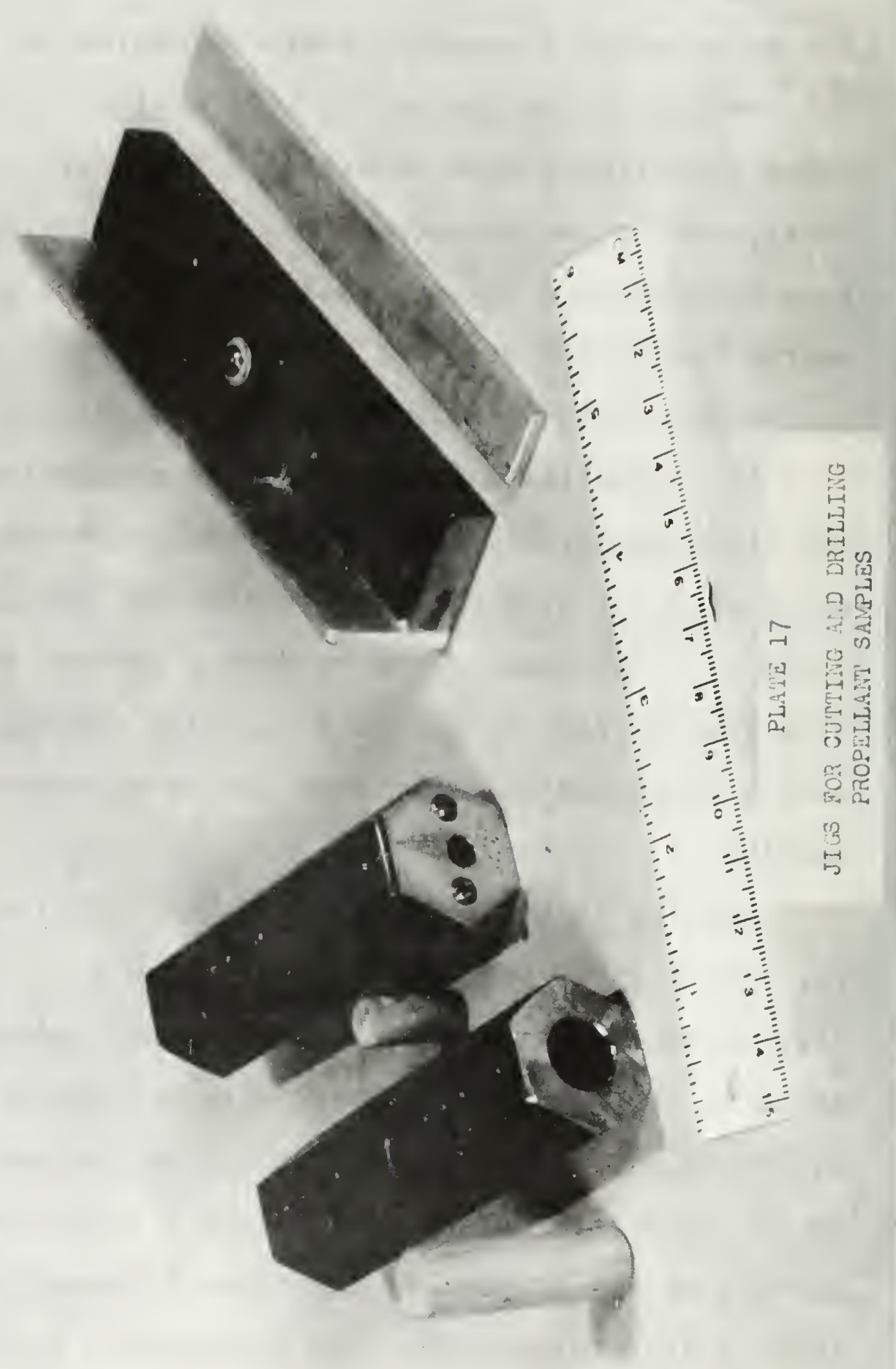


After numbering, the length of each sample was measured with a micrometer caliper or a 2 to 3 inch micrometer. Repeated measurements indicated that the measured lengths were accurate to plus or minus 0.002 inch. The height and width of the square cut strands were measured at each end with a scale graduated in 50ths of an inch. The length and average height and width were recorded.

With the exception of a few early test specimens, a cap of high-temperature epoxy (Hetron 197) was cast on one end of each sample. A piece of $3 / 4$ inch wide masking tape wrapped around the end of the sample provided the walls for the form. After curing at room temperature, the cap was sanded square on a disc sander, the final cap thickness being $1 / 8$ to $\frac{1}{4}$ inch. Thus each test specimen was similar to a small end-burning motor with a port-to-throat area ratio of one. The "motor case" was paper phenolic tube or polyester resin, and the epoxy cap served as the head-end closure.

The samples were prepared with a rigid inhibitor and end closure for two reasons: to provide mechanical support for the visco-elastic propellant in the anticipated high acceleration environment, and to retain any non-gaseous residue that might remain at the end of burning.

The samples were stored in a hazard-safe oven at ambient temperature $\left(10\right.$ to $\left.25^{\circ} \mathrm{C}.\right)$. Samples of the particular propellant being investigated were stored in a refrigerated oven at $20^{\circ} \pm 1^{\circ} \mathrm{C}$. 
Attachment to Strand Holder

The timing wires were threaded through the holes in the sample, and then secured to brass \#6-32 terminals on the strand holder using brass hex nuts. The sample was held in place by masking tape wrapped around the sample and the body of the strand holder. Samples to be used without timing wires were simply taped to the strand holder and the ignition wire installed.

The \#32 nichrome ignition wire was attached to terminals similar to the timing wires, with its mid-span touching the end of the propellant. In some experiments, the ignition wire was placed on the end of the inhibitor case, $1 / 32$ to $3 / 32$ inch away from the propellant. In either case, the propellant surface was coated with approximately $50 \mathrm{mg}$ of FFFg black rifle powder granules for rapid flame propagation. The binder used to adhere the granules to each other and to the propellant surface was LePage's Model (A) Airplane cement. One drop of cement thinned with acetone was adequate for about $750 \mathrm{mg}$ of powder.

A completed specimen (without timing wires) ready for insertion in the bomb is shown in Plate 6 . Up to seven specimens as shown in Plate 6 were prepared in advance and temporarily stored in the refrigerated oven at $20^{\circ} \mathrm{C}$. 


\section{EXPERIMENTAL PROCEDURE}

Instrumentation was turned on approximately thirty minutes before the first experiment of the day to allow time for warm-up. At the end of this period, pressure transducer excitation was set at $28 \mathrm{v}$. d.c. using the Weston Model 911 voltmeter, and transducer output was checked at a pressure approximately equal to the anticipated test pressure, either 500, 1000 , or 1500 psia. This was accomplished by connecting the charging hose directly to the bomb using a coupler fitting with the poppet valve removed. Hence there were no flow restrictions between the transducer port and the appropriate gauge at the charging station.

After completion of the transducer calibration an ASTM thermometer was inserted in the bomb where it remained for approximately three minutes. At this time, the zero psig transducer output was read and recorded.

The first propellant sample was then removed from the refrigerated oven and installed in the bomb. For acceleration levels greater than $10 \mathrm{G}$ the strand holder was installed with the slab in a horizontal plane as shown in Plate 7. For acceleration levels at or below 10G the propellant specimen was taped on the slab at an angle with the aid of a protractor and information from Figure 12, and the strand holder was installed with the slab in a vertical plane. Thus the strand axis was aligned with the anticipated acceleration vector. 
Continuity of the ignition and timing wires was checked and the temperatures inside the bomb and at the control station (ambient) were recorded.

The rotor discharge valve was closed, the discharge line removed, and the charging hose connected to the charging valve on the rotor. As the pressure in the charging hose was brought up to the surge tank pressure, the ball valve was opened slowly. This permitted fresh nitrogen to enter the bomb as well as the surge tanks. The rotor system was pressurized to a gauge pressure, as read on the appropriate gauge at the charging station, which would give the desired absolute pressure at the acceleration anticipated for that particular experiment. Thus

$$
\text { psig }=\text { psia }-15-\Delta P+8
$$

where

$$
\begin{aligned}
\text { psig }= & \text { gauge pressure read at charging station } \\
\text { psia }= & \text { desired absolute pressure in the bomb for } \\
& \text { the burning rate experiment } \\
15(\text { psia })= & \text { approximate atmospheric pressure } \\
\Delta \mathrm{P}= & \text { correction for centrifugal force } \\
& \left(8 \frac{1}{2} \% \text { at } 2000 \mathrm{G} \text { - see Appendix II }\right) \\
8(\text { psi })= & \text { pressure differential required to unseat } \\
& \text { the rotor charging valve }
\end{aligned}
$$

With the rotor system charged to the desired gauge pressure, the charging hose was depressurized and disconnected from the rotor.

After starting the engine, the transmission was engaged and the test cell vacated. 
After recording the transducer output voltage the centrifuge was brought up to the desired speed as indicated on the EPUT Meter, the timers set at zero, and the pressure transducer output trace located near the left-hand edge of the Visicorder chart. The strand ignition switch was then held on until the pressure signal indicated ignition. Centrifuge RPM was closely monitored as long as the strand was burning. RPM did not vary more than plus or minus two. At the end of burning as indicated by the pressure trace, the throttle was closed, engine ignition turned off and the centrifuge allowed to coast to a stop. Timer readings and the average EPUT Meter reading were recorded on the data she et.

The Visicorder chart span was adjusted to give approximately a five inch displacement of the pressure trace from the beginning to the end of burning. The trace was initially set about one inch from the left hand edge of the paper. Chart speed was generally one inch per second. For some runs where burning rates greater than $0.5 \mathrm{in} / \mathrm{sec}$ were expected the chart speed was $2 \mathrm{in} / \mathrm{sec}$. On some runs where the burning rate was expected to be less than $0.15 \mathrm{in} / \mathrm{sec}$ the chart speed was 0.8 or $0.4 \mathrm{in} / \mathrm{sec}$. The timer was set to give time lines at the rate of one per second.

The chart was turned on approximately two seconds before the strand was ignited. This was to insure the appearance of at least one time line on the chart before ignition. Approximately two seconds after the end of burning, as 
indicated by the trace on the chart, the chart was turned off. This record was then cut off of the remaining roll of paper and the following information added: run number, date, propellant designation, pressure, $\mathrm{N}(\mathrm{RPM} / 2)$, and sample number.

After the centrifuge had coasted to a stop, the transmission was disengaged and the rotor moved to a position where the discharge line could be connected to the discharge valve. The surge tanks were then isolated from the bomb, the bomb depressurized and the strand holder removed. An industrial vacuum cleaner suction hose was inserted in the bomb for purging and cooling.

The vacuum cleaner was allowed to run for about three minutes. After removal of the vacuum cleaner hose, the thermometer was inserted in the bomb.

While the bomb was being cooled with the vacuum cleaner, the inhibitor case was removed from the strand holder, marked with the run number, and the open end sealed with masking tape. The inhibitor cases were saved for future analysis of possible residues retained at the end of burning.

The strand holders were cleaned in cold water with a laboratory bottle brush and dried using an air hose. Comments on Experimental Methods

Initial experiments using the $\mathrm{X} 104$ and $\mathrm{X} 304$ propellants were carried out employing both the electric timers and the pressure-time history to calculate average burning rate. Number 32 (0.008 in. dia.) nichrome wire was initially used 
for timing wires. However, it was found that although the wires melted as the flame front passed, there was a sufficient amount of condensed phase combustion products retained on the inside walls of the inhibitor case to conduct a current. In many cases this current was sufficient to hold the timing relays in the actuated position. The resistance across the timing wire terminals after a test varied from $100 \Omega$ to open circuit. Although the relay holding coils, which have a nominal resistance of $250 \Omega$, are advertised as $12 \mathrm{v}$., it was found that all of six relays on hand would close with 4.5 volts or less, while dropout voltage varied from 2.3 to 0.5 volts. Hence the effect of the passing flame was not to break continuity as in the case of more conventional strand burning experiments, but merely to insert a resistance in the circuit; this resistance was generally too low to result in actuation of the relays.

In an attempt to solve this problem, \#32 copper wire was used, the thought being that its lower melting point would permit the wire to melt away from the conducting residue in the inhibitor case. The performance of the copper wire was no better than the nichrome.

A second attempt to solve the problem was made using \#32 Pyro-fuse. The Pyro-fuse wire was composed of an aluminum core sheathed in palladium. At $600^{\circ} \mathrm{F}$, the metals alloyed rapidly and exothermically. With the Pyro-fuse timing wires the electric timer performance was reliable. 
The use of timing wires was found to be excessively tedious due to the time required to drill holes, insert the timing wires and make the necessary electrical connections. In addition, the pressure-time history indicated that the timing wire clearance holes acted like small voids in the propellant. This was aggravated at higher accelerations.

A comparison between the average burning rates obtained from the electric timers and the pressure-time history showed agreement generally within $2 \%$. In view of this agreement and the above mentioned disadvantages, the timing wires were not used after the first 75 experiments.

The head end of the propellant specimens used early in the program was capped with a double thickness of masking tape. This proved effective up to about 50G. At higher accelerations, the hot residue burned through the tape and escaped into the bomb. A cap of APCO 210 Resin and 180 Hardener was then cast on the end in order to retain the hot residue. This proved to be adequate at 200G and below, but not above 200G. The caps of Hetron 197 high-temperature epoxy described in the section on preparation of propellant samples were found to be effective at all levels of acceleration up to $2000 \mathrm{G}$.

The first strand holders used in the program had slabs made of silicone glass laminate (Budd Co. Dilecto Grade 607). This material was found to have the best combination of strength, dielectric and high-temperature properties of the 
several commercial laminates investigated. However, protection of the slab from the hot combustion flame remained a problem.

A remarkably effective insulator was found to be a $3 / 32$ in. thick phenolic plastic insulation sheet (FSN 9330282 5641). Contact with the propellant flame caused surface charring which was easily washed away with a brush and cold water. The insulation sheet would last for about 20 experiments after which it was easily replaced. It was found that with the phenolic insulation sheet between the flame and the slab, the high temperature properties of the silicone glass were not required. Subsequent strand holders were made with canvas phenolic slabs and strand supports.

The rapid establishment of a uniformly burning surface across the entire cross-section of the strand was believed to be necessary because of the relatively short samples being used. In order to achieve this goal, the ignition wire was laid against the propellant across the middle of the cross section and a layer of FFFg black rifle powder granules spread across the propellant surface. Thus ignition would take place in the center of the strand face and proceed outward in both directions. It was found during the experimental program, however, that the location of the ignition wire had a significant effect on the burning rate of the non-aluminized propellants at low accelerations. Hence for the remainder of the program the ignition wire was laid on the inhibitor case $1 / 32$ to $3 / 32$ inch from the propellant. 
FFFg black rifle powder was used not only to cover the face of the propellant but also to connect this covering to the ignition wire. Thus the ignition wire was out of the exhaust flame and remained intact throughout burning. The pressure - time histories indicate rapid ignition regardless of ignition wire location.

\section{DATA REDUCTION}

\section{Determination of Burning Rates}

Average burning rates for the propellants between the timing wires were calculated by dividing the distance between two adjacent wires by the elapsed time indicated by the timer. The three values thus obtained were averaged to obtain the average burning rate.

Determining average burning rate from the pressure time history was more involved. Burning was assumed to commence with ignition of the black powder. This appeared as a step input on the chart and was well defined. The end of burning was assumed to occur at the point of maximum pressure. The pressure in the system decreased after burning due to gas cooling. This point was not always well defined. In these instances the method of tangents was used to define the end of burning.

Small variations in the Visicorder chart speed were observed from one run to the next. However, the frequency of the time lines was constant at 0.965 lines/second. This was 
determined by putting a 60 cycle signal into the recorder and counting the number of input signal peaks between time lines.

The number of time line intervals between ignition and end of burning was determined by adding the fractional intervals at the beginning and end to the number of whole intervals in between. The fractional intervals were determined with a scale graduated in 50 ths of an inch. The necessary assumption was that the chart speeds were constant throughout the time line intervals in which ignition took place and burning ended.

Total elapsed burning time $t_{b}$ was found from

$$
t_{b}(\mathrm{sec})=\underset{\text { total time line intervals } /(0.965 \text { inter- }}{\text { vals } / \mathrm{sec})}
$$

and the average burning rate $r$ was found from

$$
r(\mathrm{in} / \mathrm{sec})=\text { sample length }(\mathrm{in}) / \mathrm{t}_{\mathrm{b}}(\mathrm{sec})
$$

Determination of Acceleration Field

At constant RPM, the acceleration field in the combustion bomb had two components: a vertical component due to the earth's gravitational field, and a radial component dependent on the radius and angular velocity. Hence the acceleration vector always remained in a vertical plane, but both direction and magnitude were dependent upon angular velocity. With the centrifuge at rest, the direction was vertical and downward. At high speed, the direction was nearly horizontal. 
The symbols used are defined as follows:

$$
\begin{aligned}
& a=\operatorname{radial} \text { acceleration } / \mathrm{ft} \text { radius }\left(1 / \mathrm{sec}^{2}\right) \\
& r=\operatorname{radius}(i n) \\
& \mathrm{N} \text { = number in EPUT meter display (RPM/2) } \\
& g=\text { acceleration due to gravity }\left(32.2 \mathrm{ft} / \mathrm{sec}^{2}\right) \\
& \begin{array}{c}
G_{r}^{\prime}=a / g= \\
\text { radius }(1 / f t)
\end{array} \\
& G_{r}=\text { radial load factor }=G_{r}^{\prime}(r / 12 \text { in } / f t) \\
& \text { (dimensionless) } \\
& G=\text { total load factor } \\
& \omega=\text { angular velocity (radians/sec) }
\end{aligned}
$$

The radial acceleration per foot radius is

$$
\begin{aligned}
a & =r \omega^{2}=(1 \mathrm{ft})(4 \pi \mathrm{N}) /\left((60 \mathrm{sec} / \mathrm{min})^{2}(1 \mathrm{ft})\right) \\
& =N^{2} /(4.775)^{2} 1 / \mathrm{sec}^{2}
\end{aligned}
$$

and

$$
\begin{aligned}
G_{r}^{\prime} & =a / g=N^{2} /\left((4.775)^{2} \times 32.2\right) \\
& =N^{2} / 734.5
\end{aligned}
$$

or

$$
N=27.1\left(G_{r}^{\prime}\right)^{\frac{1}{2}}
$$

At a radius of 35.6 inches (the mid-point of the propellant sample) the radial load factor is

$$
\begin{aligned}
G_{r} & =(35.6 " /(12 \mathrm{in} / \mathrm{ft})) G_{r}^{\prime} \\
& =N^{2} / 247.5
\end{aligned}
$$

and $\quad N=15.72\left(G_{r}\right)^{\frac{1}{2}}$

For radial load factors of 10 or more, the contribution of the vertical component is less than one percent. Hence for 
$\mathrm{G}_{r}>10$, the acceleration vector $\mathrm{G}$ is assumed to be horizontal and equal to $G_{r}$. That is,

$$
G=N^{2} / 247 \cdot 5
$$

and

$$
N=15.72 G^{\frac{1}{2}}
$$

This solution is plotted in Figure 10.

For $\mathrm{G}_{\mathrm{r}} \leq 10$, the vertical component is taken into account. The solutions are shown in Figures 11 and 12 .

Figures 10, 11, and 12 were used to select the value of $\mathrm{N}$ to obtain the desired acceleration. The recorded value of $\mathrm{N}$ was used to calculate $\mathrm{G}$ from Equation (21).

For experiments at or below $G_{r}=10$, Figure 12 was used in conjunction with Equation (21) to calculate G.

\section{Experimental Error}

The primary means for determining average propellant burning rate utilized the pressure-time history and the propellant strand length. The pressure-time history traces on the charts were at least five inches long, and were measured to within \pm 0.1 inch. The propellant strands were at least 2.125 inches long and were measured to within \pm 0.002 inch. Hence the probable uncertainty in burning rate measurement is within $\pm 2.1 \%$. The secondary means for determining average burning rate utilized timing wires and electric timers. Three adjacent timing intervals were each at least one-half inch long, and the 0.025 inch diameter timing wire holes were located within \pm 0.0005 inch. The electric timers indicated elapsed times of at least $1.5 \mathrm{sec}$, and could be 


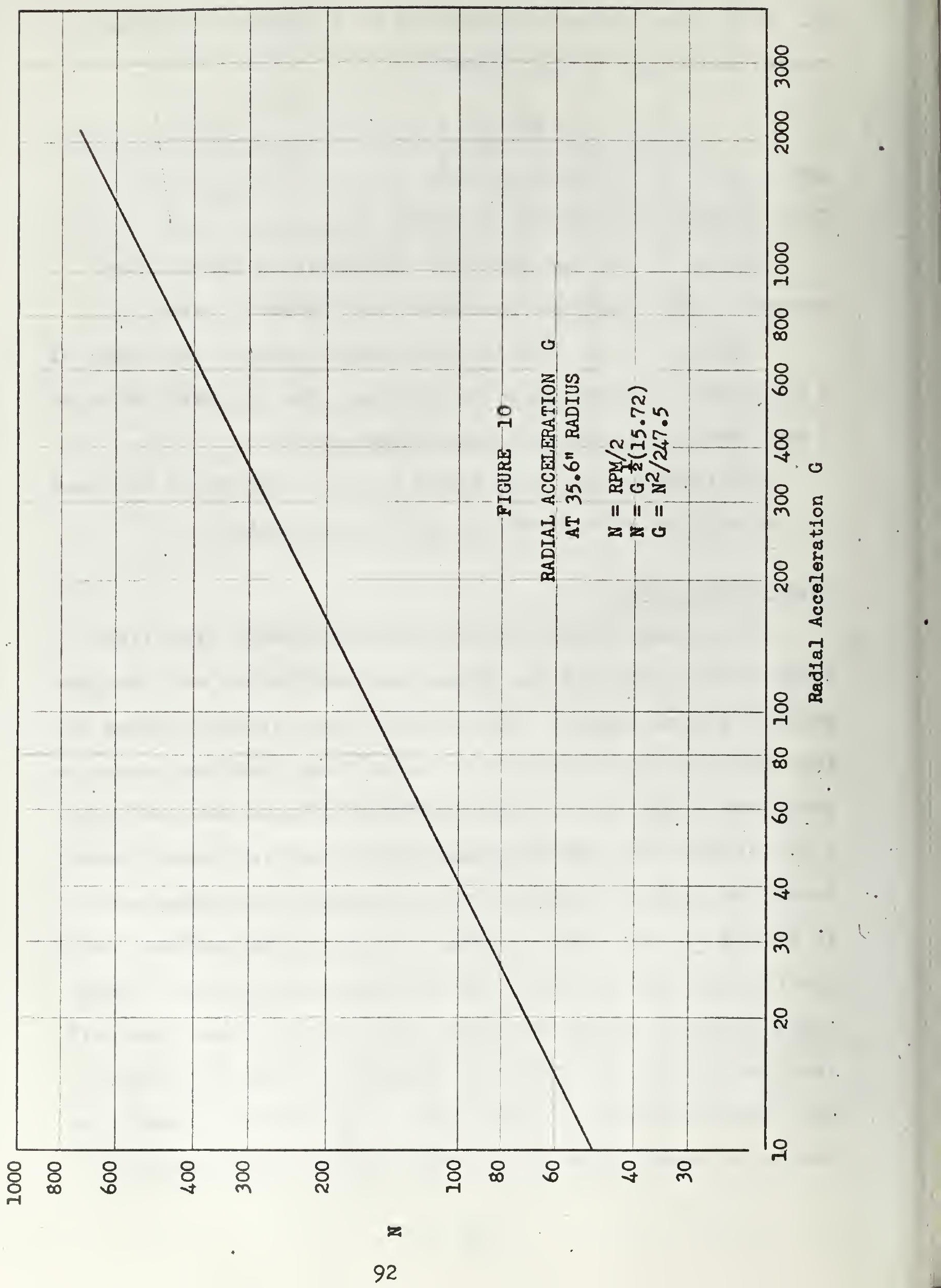




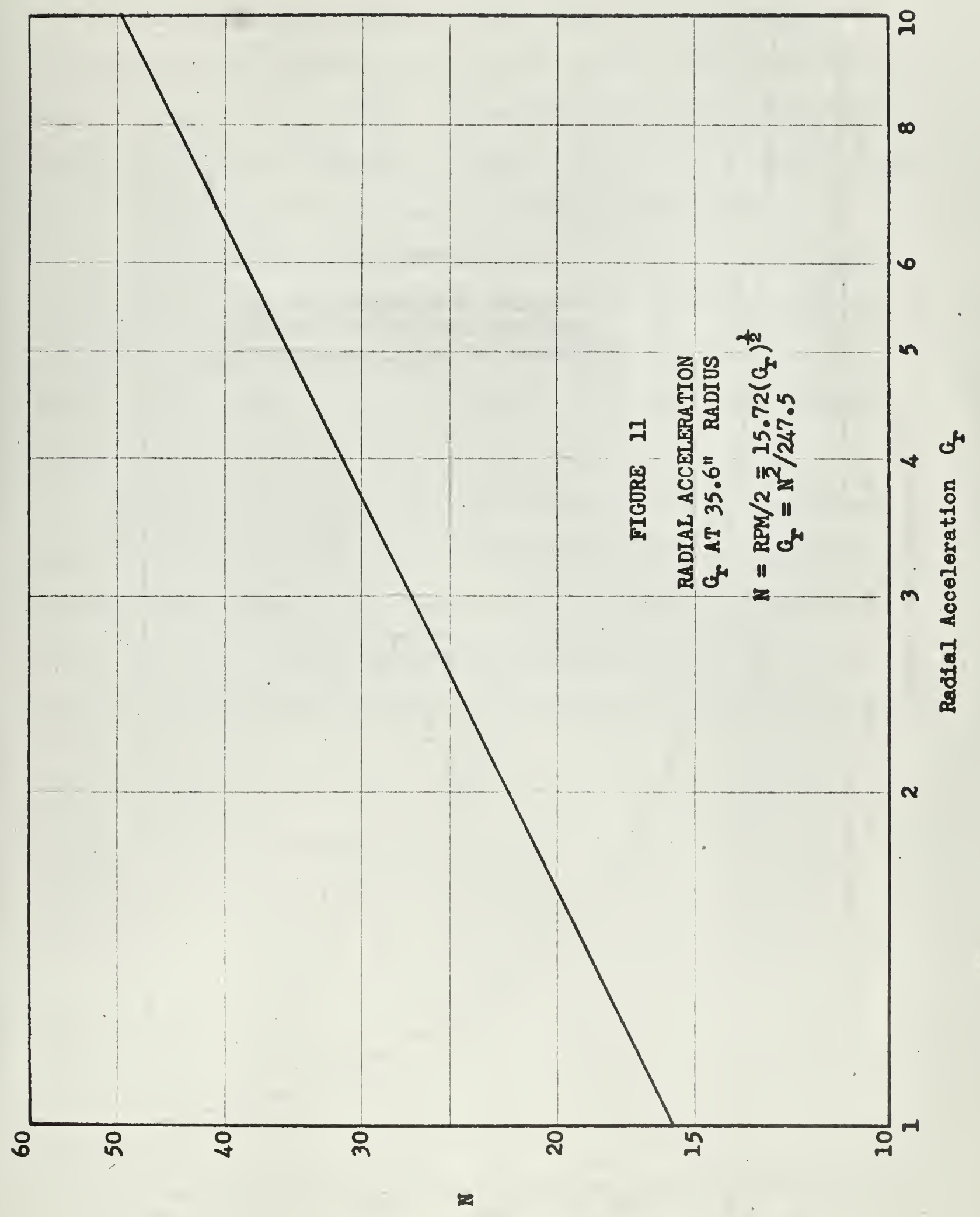




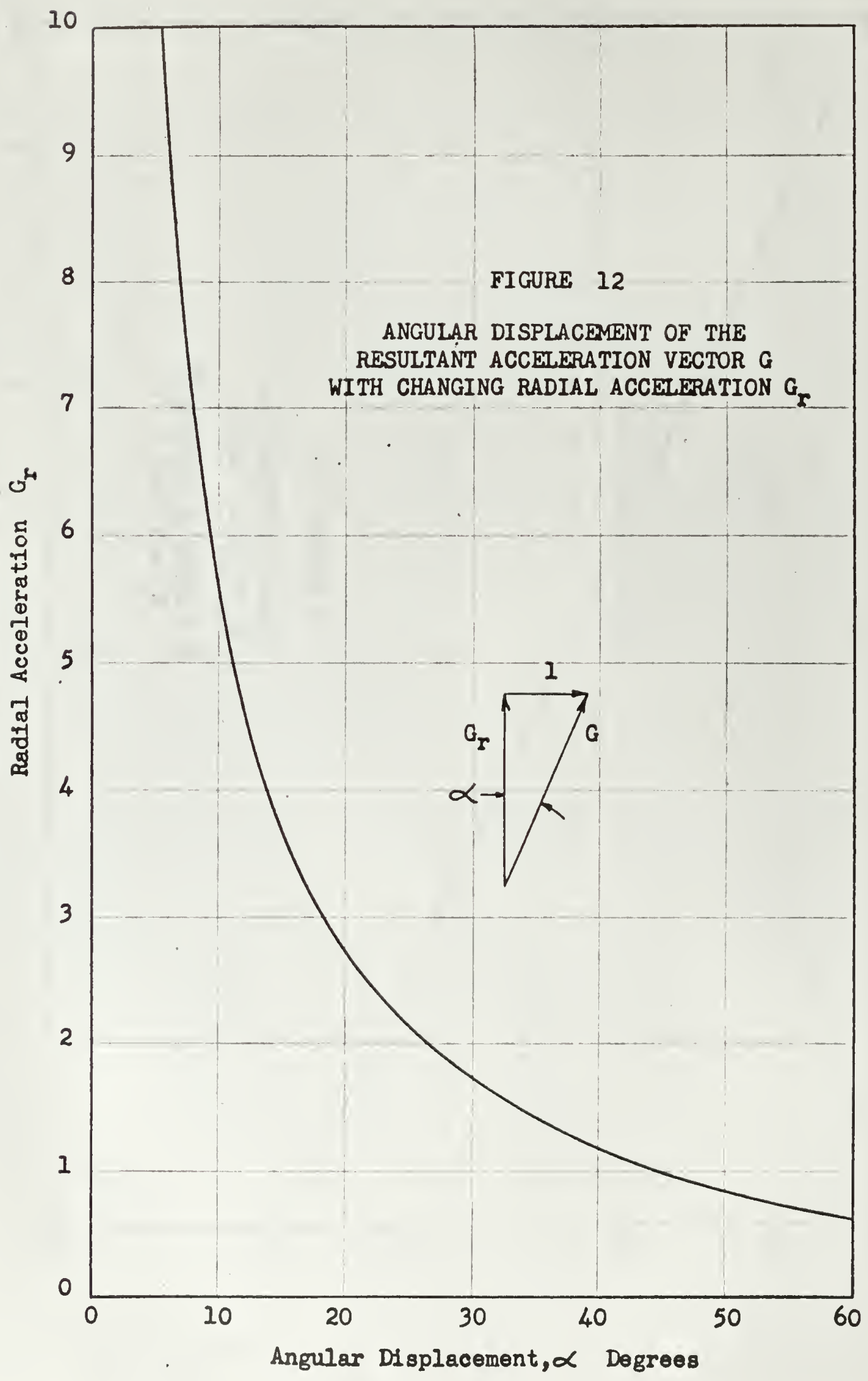


read to $\pm 0.01 \mathrm{sec}$. Assuming the total uncertainty in wire spacing to be \pm 0.025 inch in the total timing distance of 1.5 inches, the probable uncertainty in burning rate measurement is less than $\pm 2.03 \%$. Repeated measurements of strand burning rates are typically within $\pm 2 \%$ of the average value for the type propellants used in this investigation. Radial acceleration is dependent on $(\mathrm{RPM})^{2}$ which did not vary more than \pm 2 RPM. At $100 \mathrm{G}$ the corresponding RPM was 314 . The average propellant sample radius was 35.6 inches $\pm 0.2 \mathrm{inch}$. Hence, at $100 \mathrm{G}$ or greater, the probable uncertainty in radial acceleration is within $\pm 1.4 \%$.

The pressure gauges used in charging the rotor system could be read to $\pm \frac{1}{4} \%$ of the full scale value. Repeated comparisons between the gauges and the pressure transducer indicated that the rotor system was pressurized to within $\pm 1 \%$ of the desired pressure at all three pressure levels. 
EXPERIMENTAL RESULTS AND DISCUSSION

Experimental results are presented for burning rate change with acceleration and pressure for three basic propellants. The effect of varying aluminum mass loading and aluminum particle size in two of the basic formulations is shown. The results are compared with the critical particle size model and the modified granular diffusion flame model discussed in Chapter II.

Results of an analysis of propellant residues using infrared spectrophotometry and $\mathrm{x}$-ray diffraction techniques are also presented.

\section{BURNING RATE EXPERIMENTS}

\section{General}

Results are presented in the form of burning rate augmentation $\left(r / r_{0}\right)$ and actual burning rate $(r)$ as a function of acceleration $(G)$. Burning rate augmentation $\left(r / r_{0}\right)$ is defined as the actual burning rate $(r)$ at a given pressure and acceleration divided by the burning rate at the same pressure with the centrifuge at rest $\left(r_{0}\right)$. Combustion bomb pressure, either 500, 1000, or $1500 \mathrm{psia}$, is the parameter.

Radial acceleration levels varied from zero with the centrifuge at rest to a maximum of 2000 times standard 
gravity (2000G). With the exception of the zero radial acceleration condition, the acceleration vector was always normal to and directed into the burning surface. That is, the burning end of the strand was toward the centrifuge axis of rotation. With the centrifuge at rest, the one G field due to the gravitational field of the earth was parallel to the burning surface.

Two different size strands were used in the X104, X200, and $\mathrm{X} 304$ propellants. The X104 and X200 strands were 0.2 inch $\times 0.2$ inch $\times 2 \frac{1}{4}$ inches and 0.4 inch $\times 0.4$ inch $\times 2 \frac{1}{4}$ inches. The $\times 304$ strands were $\frac{1}{4}$ inch diameter and $3 / 8$ inch diameter. In general, squares denote data obtained using the larger strands, and circles denote data obtained using the smaller strands.

Each datum point is the result of one experiment. Curves are drawn to indicate the apparent trend of the data. These curves are used to facilitate comparison of results. Results for the $\mathrm{X} 100$ series propellants are shown in Figures 13 through 36 . The $\mathrm{X} 200$ propellant results are shown in Figure 37, and the $X 300$ series are shown in Figures 38 through 57.

$\underline{\mathrm{X} 100 \text { Series - Polyurethane Binder }}$

X101 - no aluminum. Experimental results for the X101 propellant are shown in Figures 13 through 20. The Figures show data points with and without an asterisk (*). An asterisk by a datum point indicates that the ignition wire did not break or melt and was still intact after the experiment. 


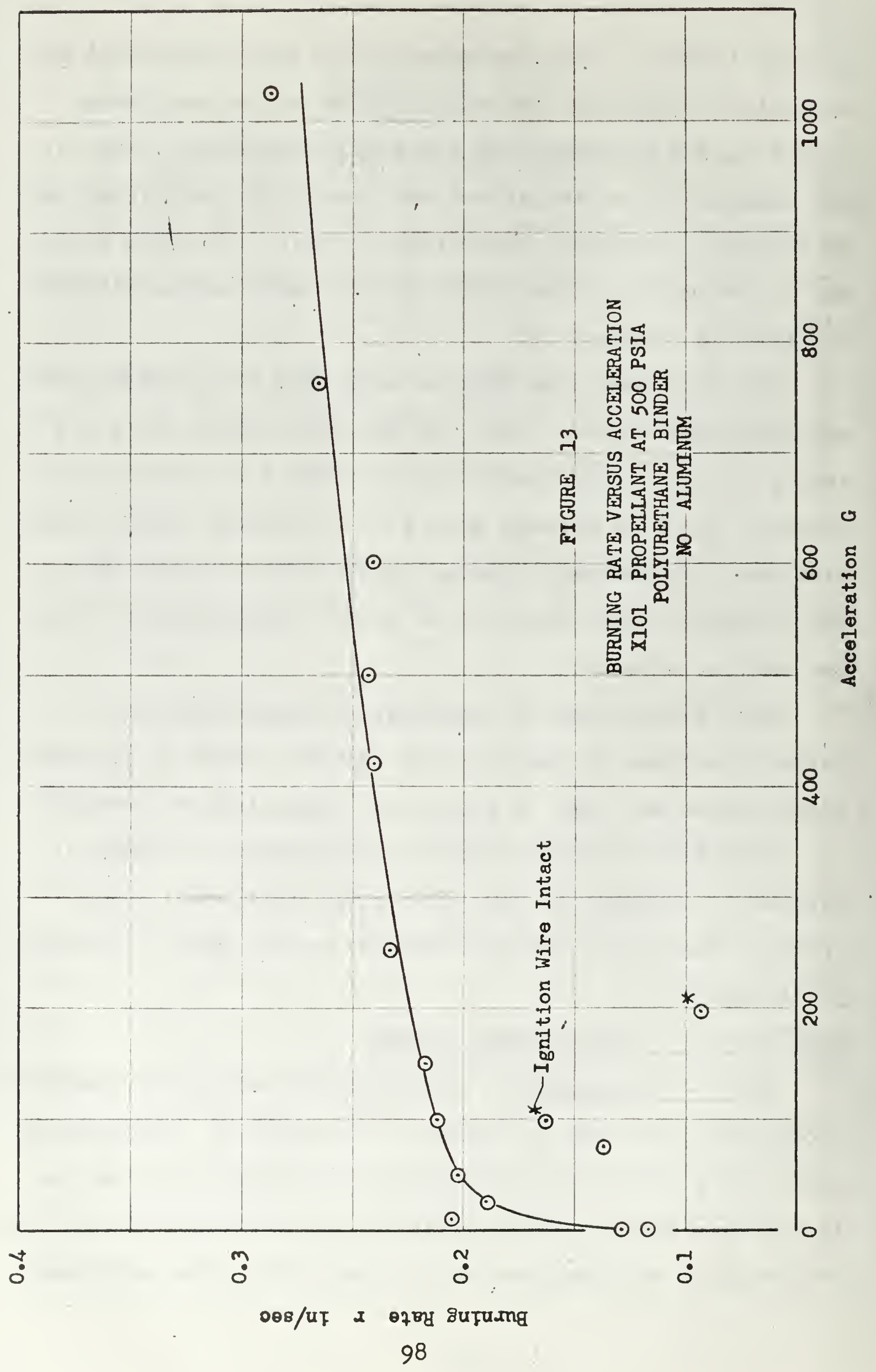




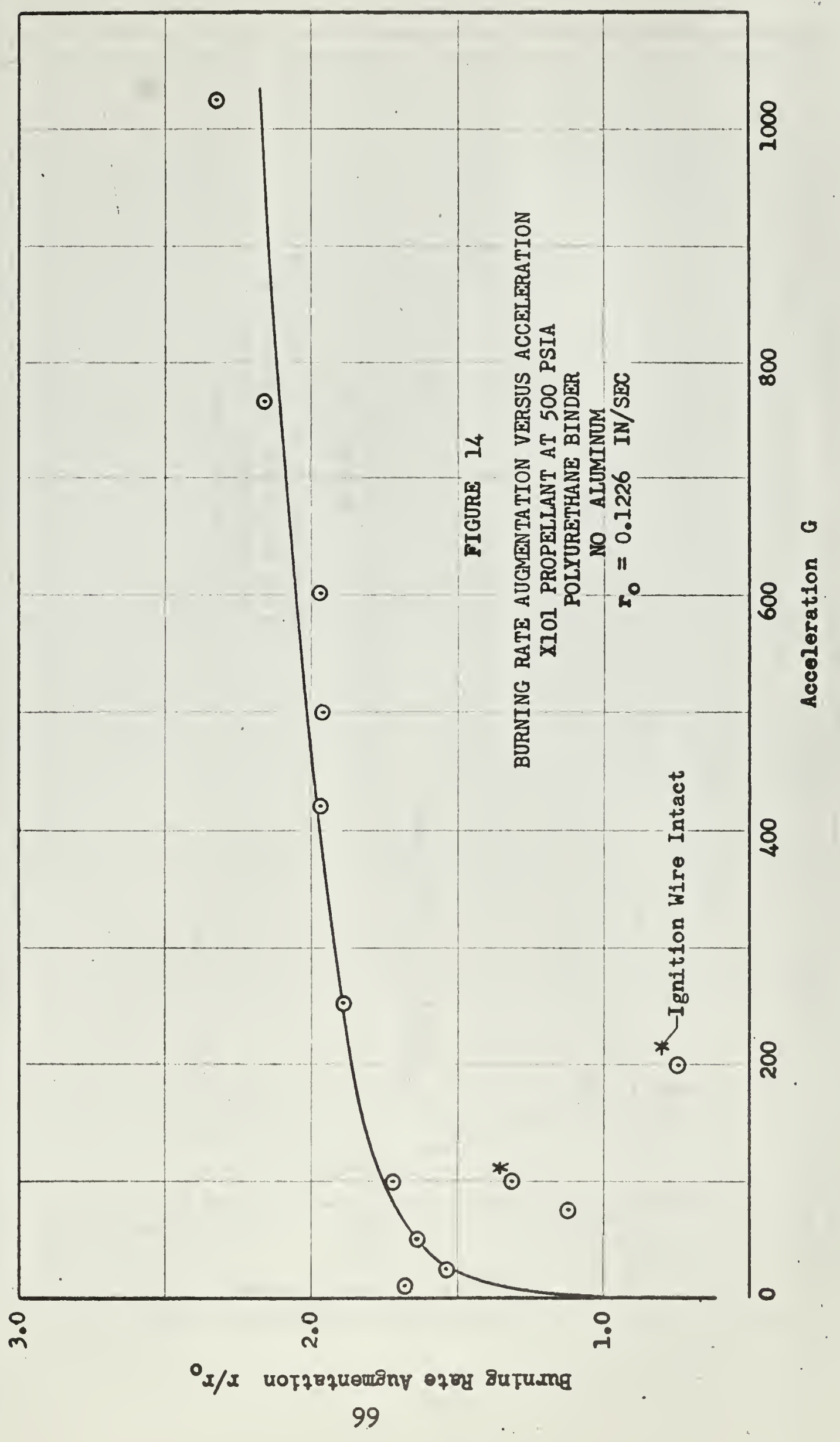




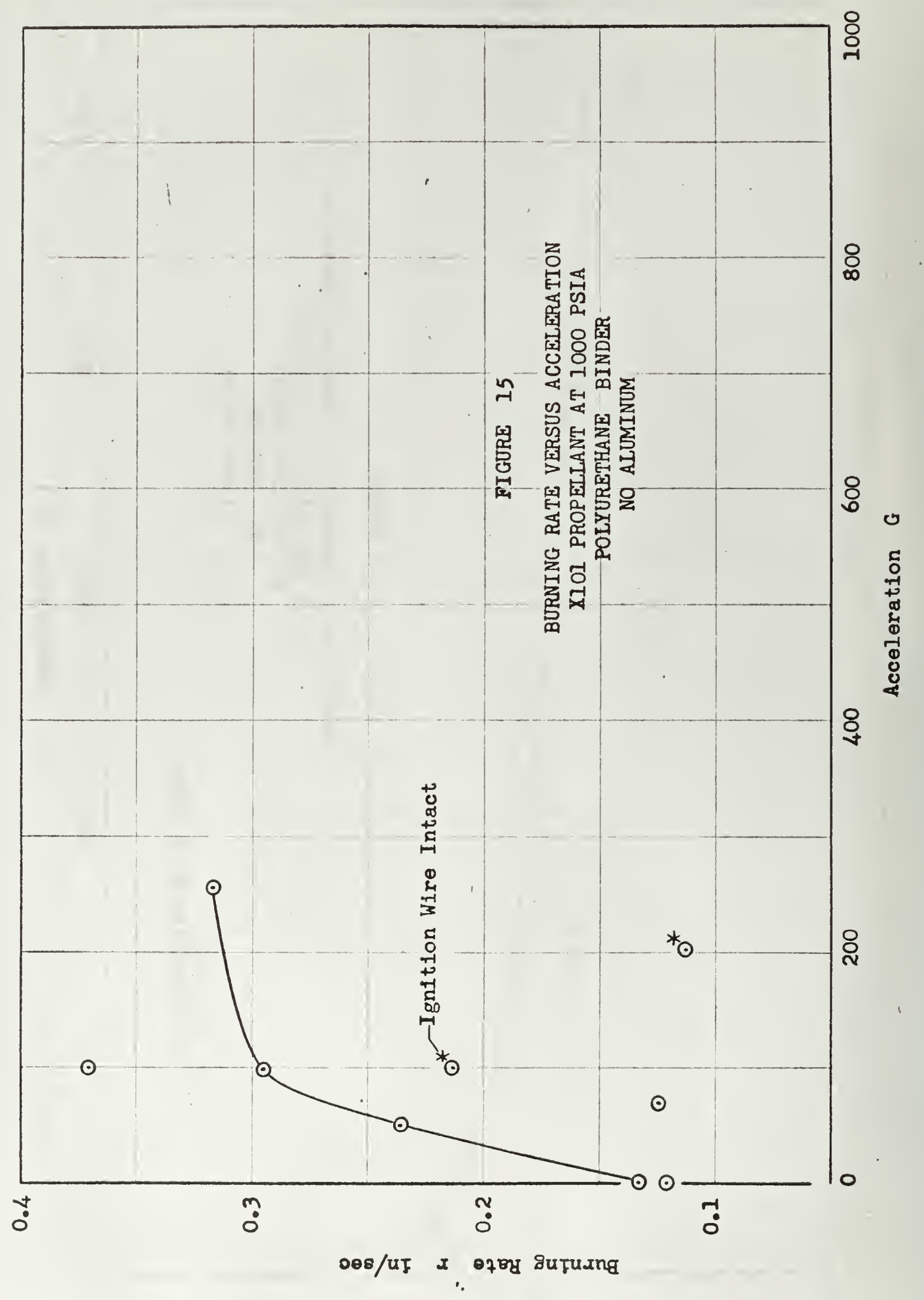




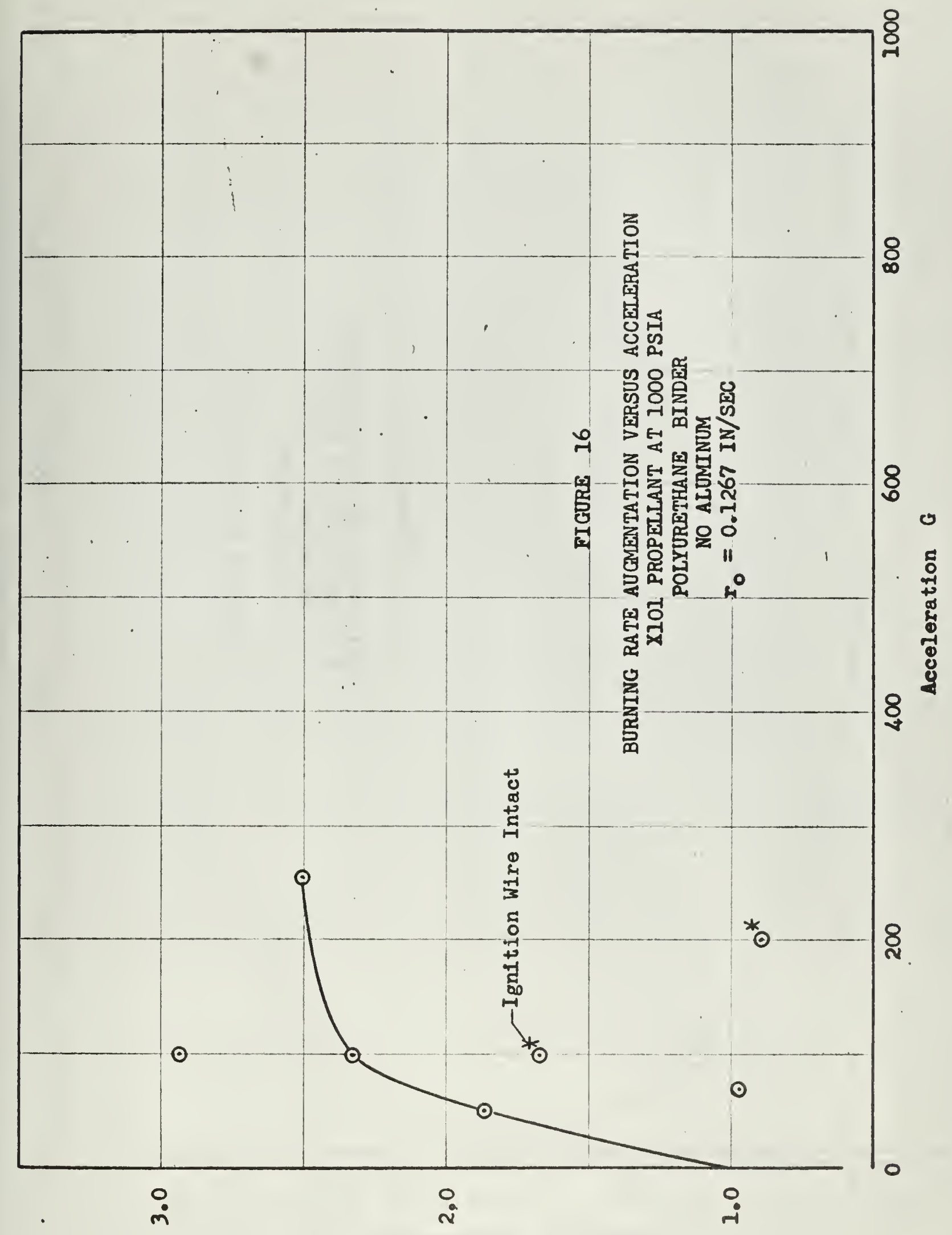

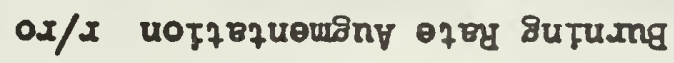




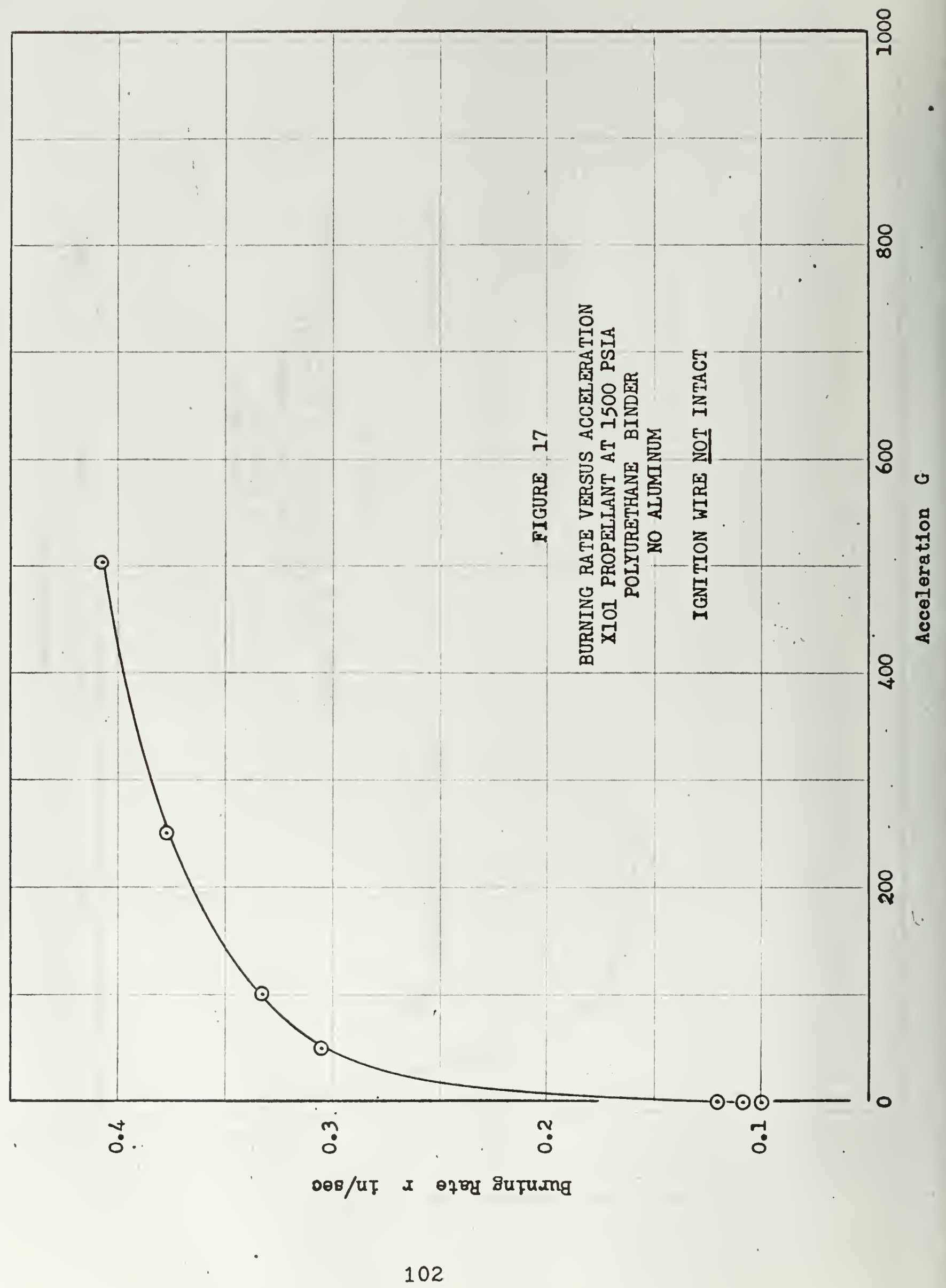




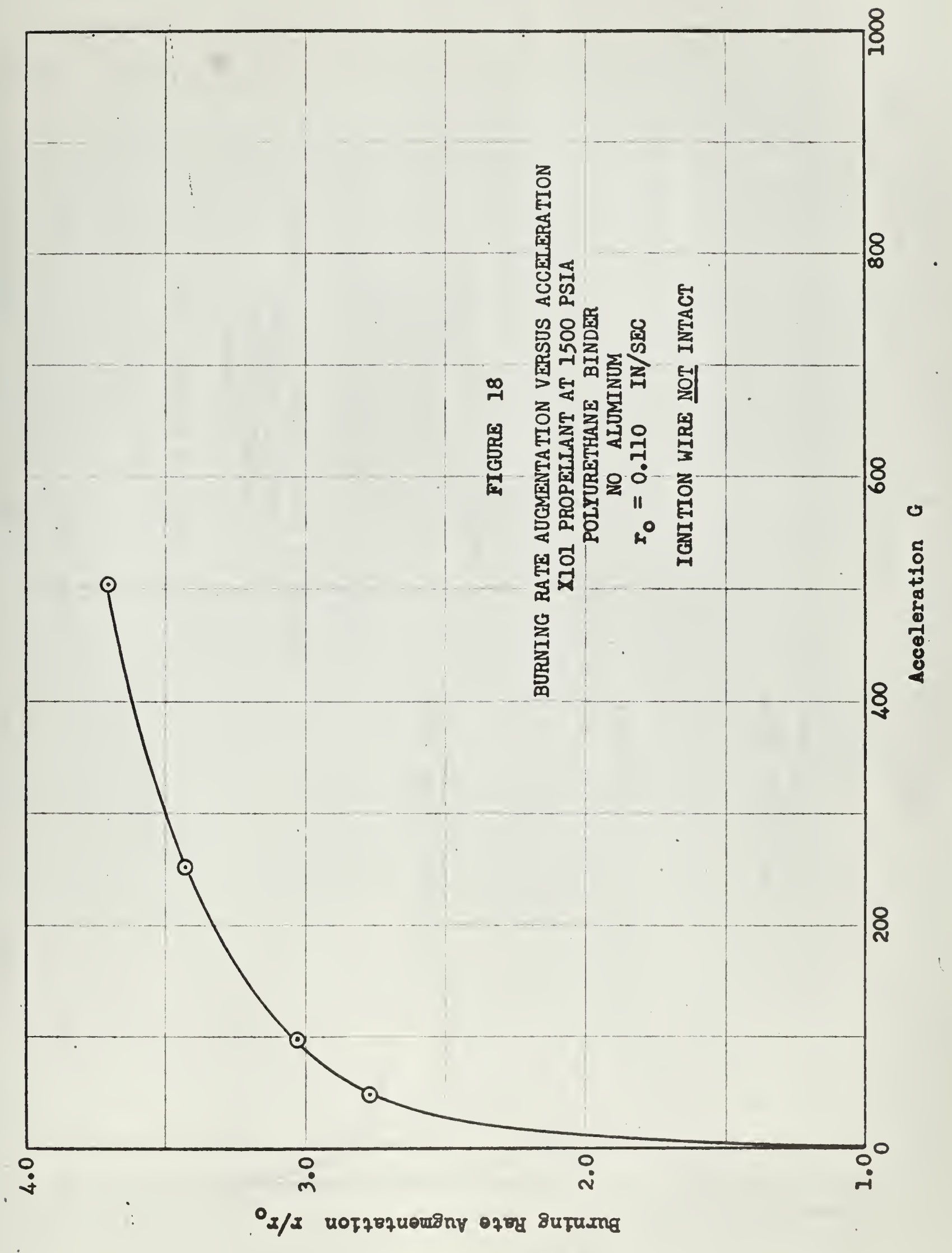




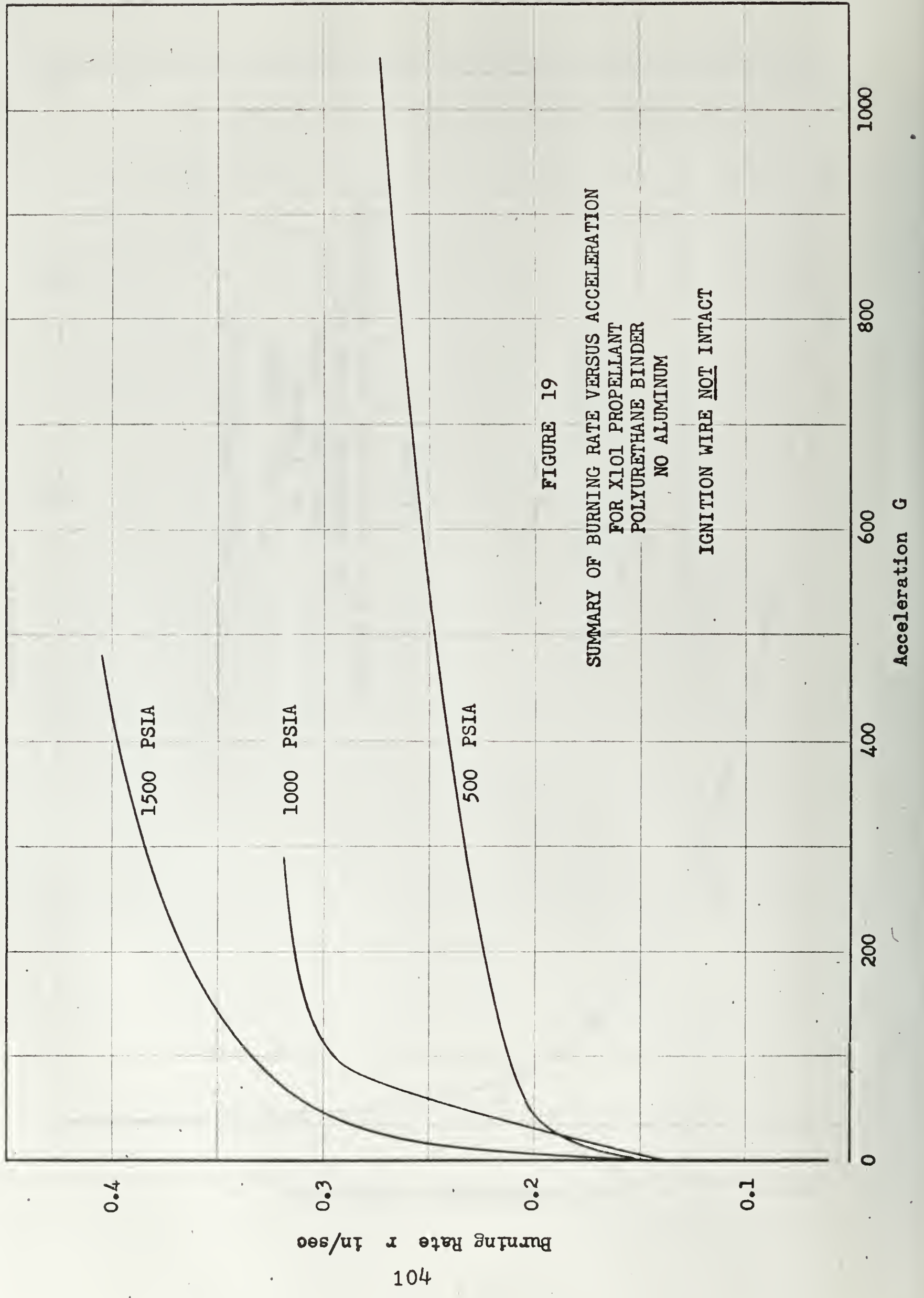




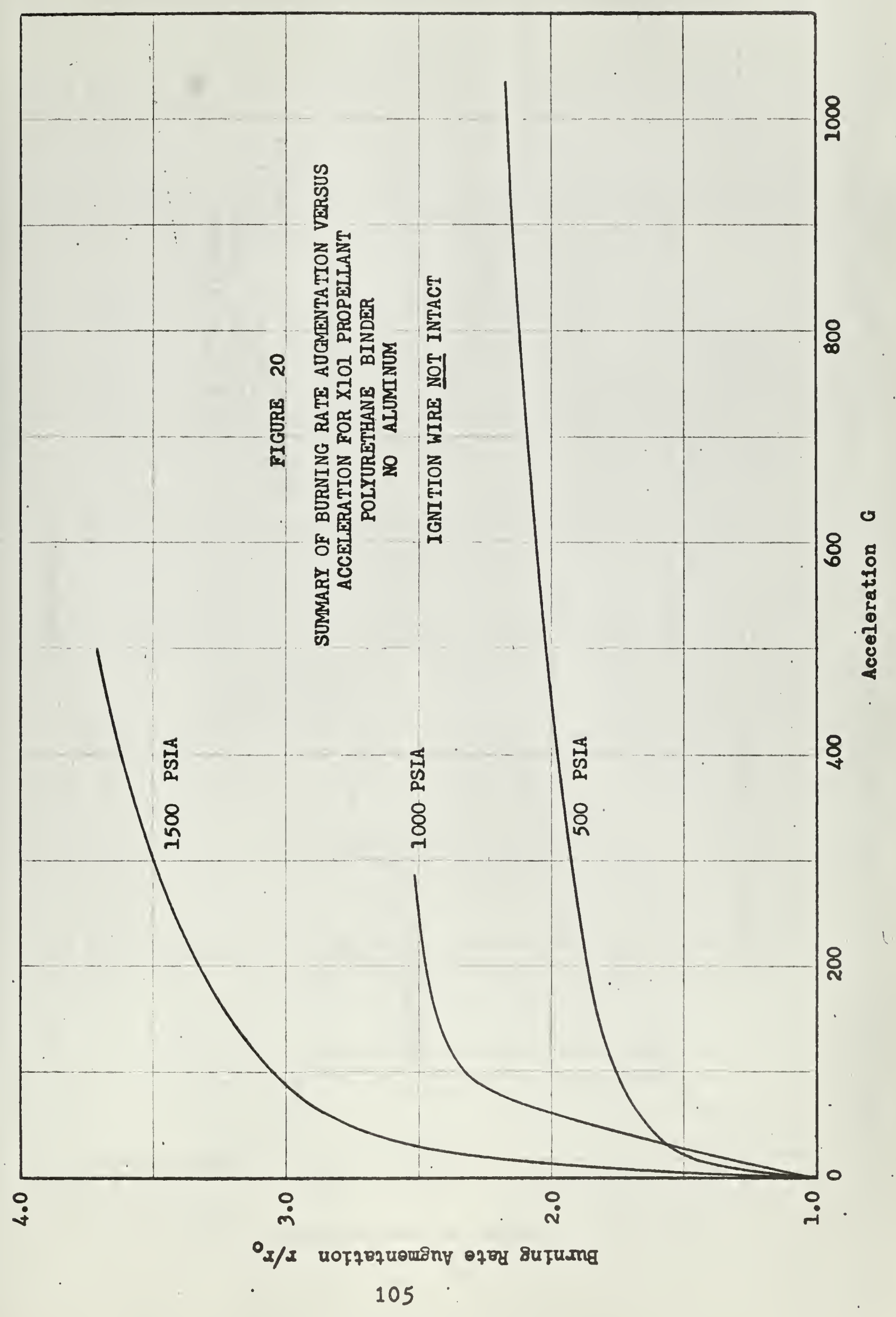




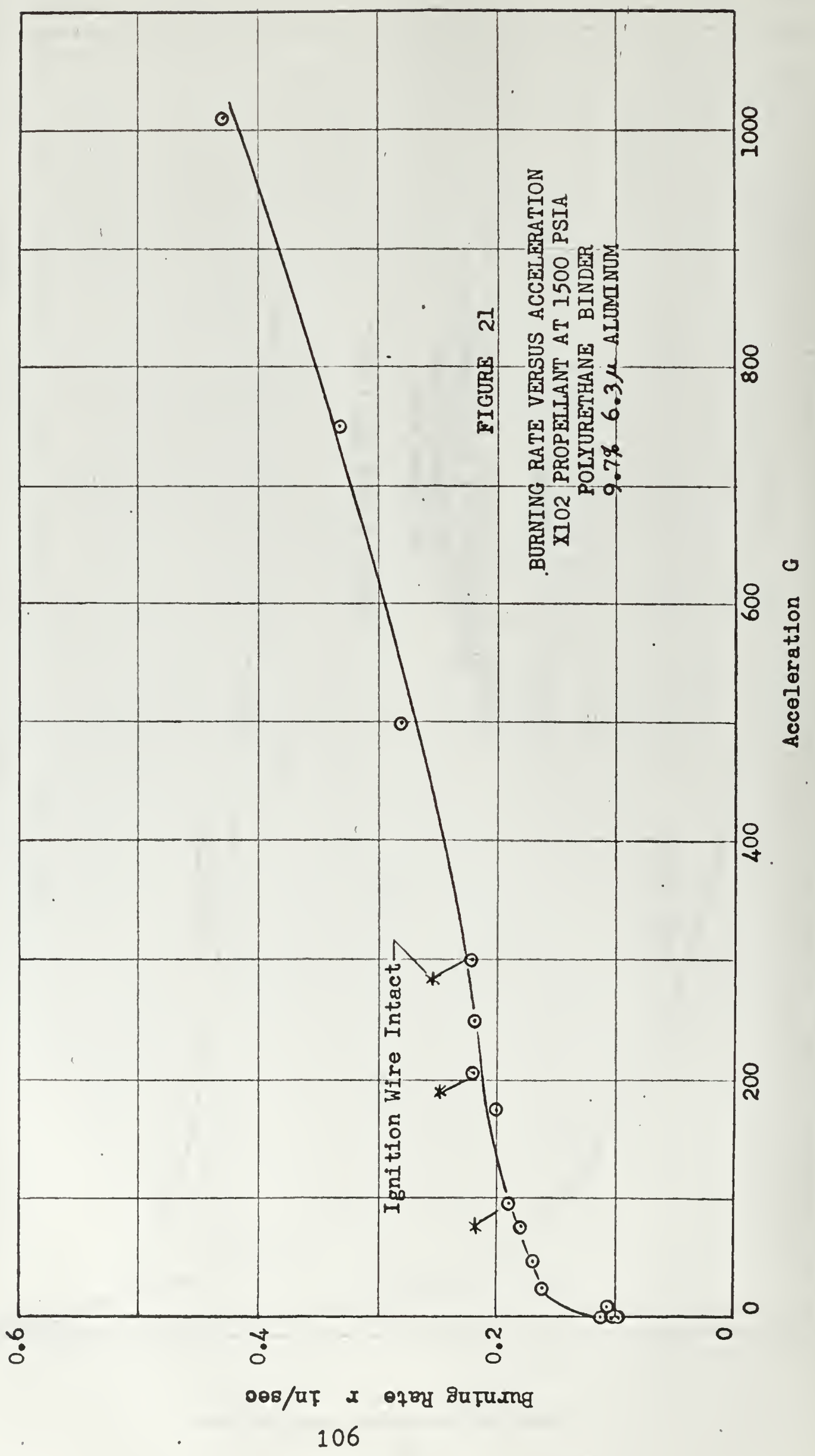




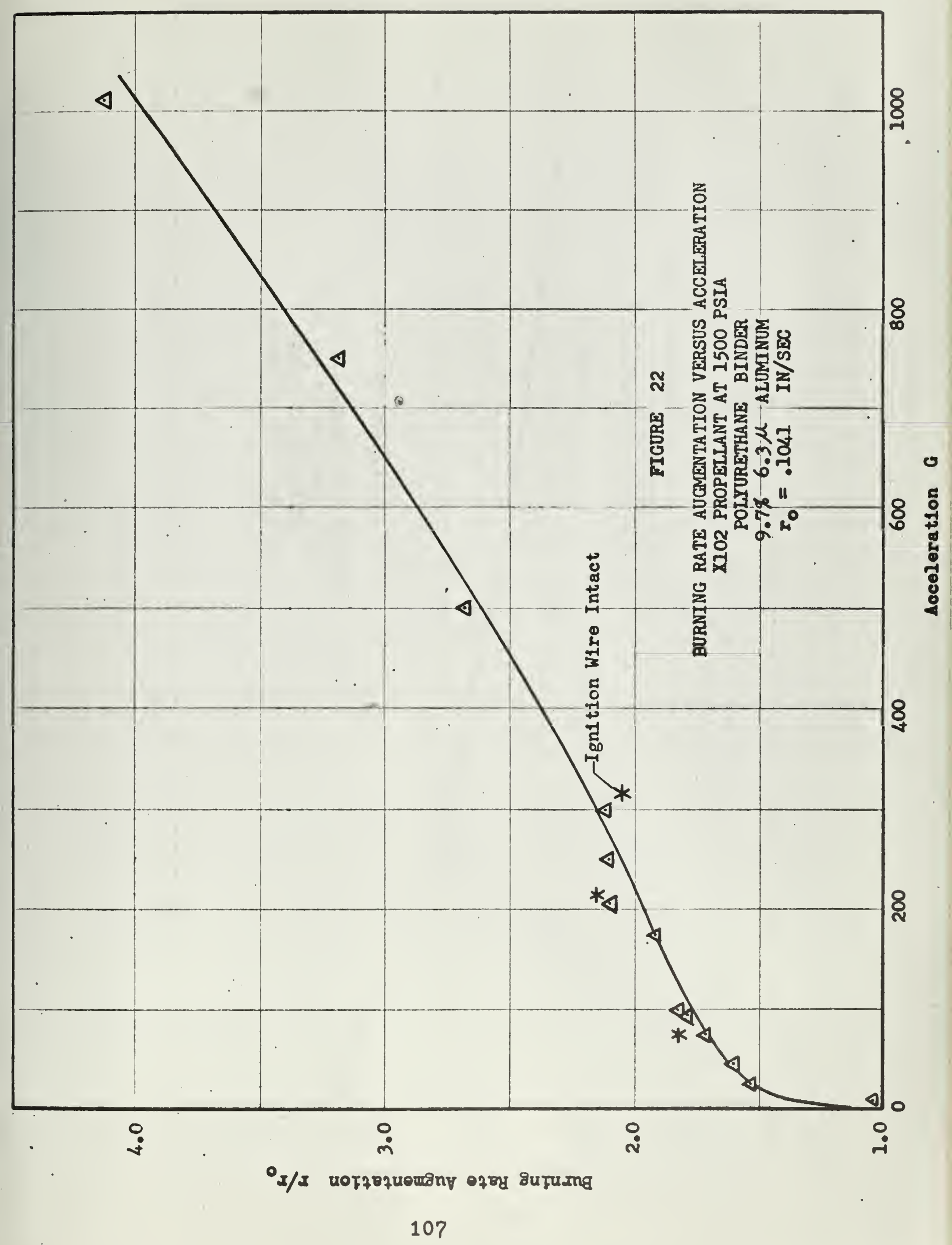




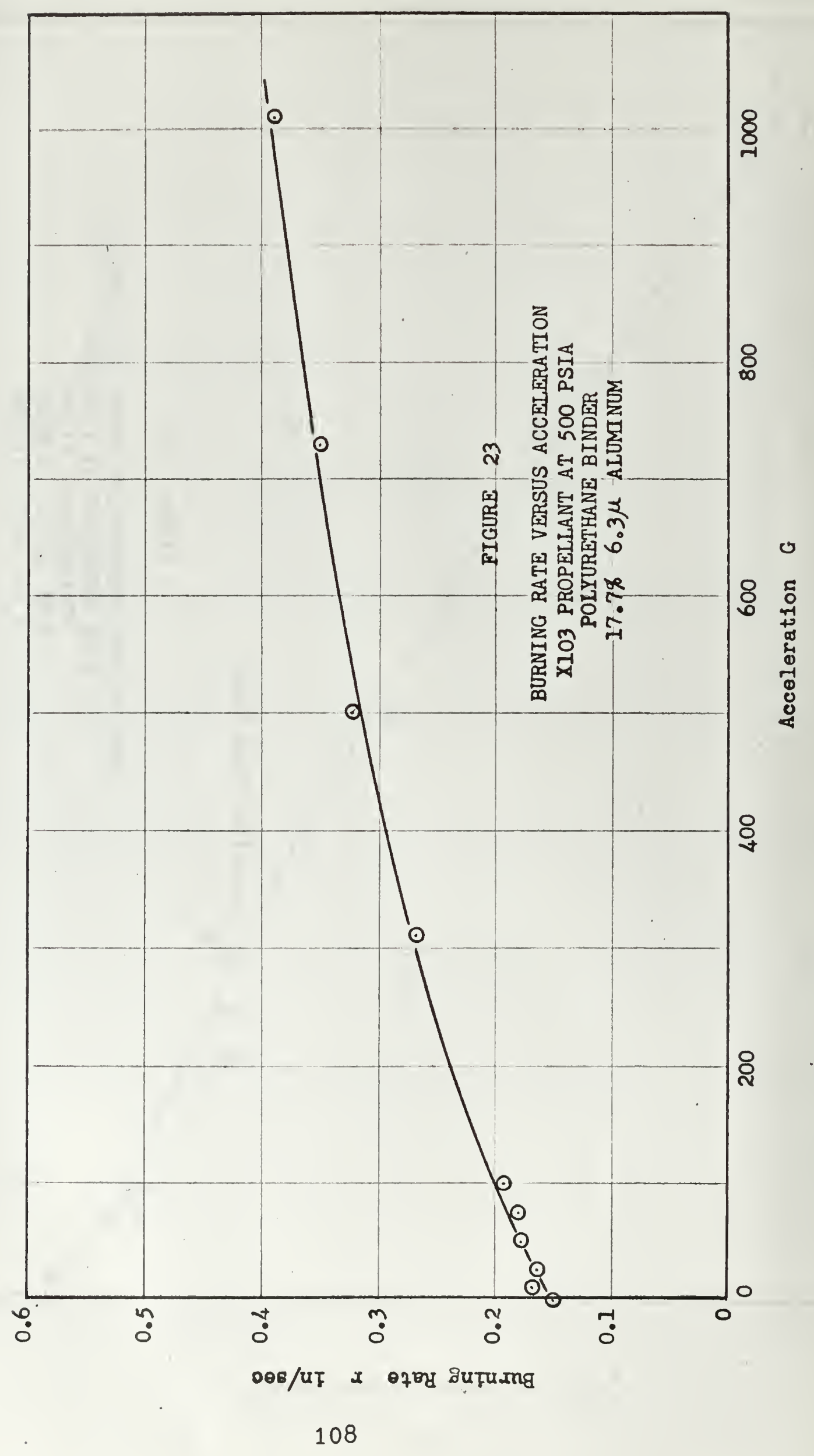




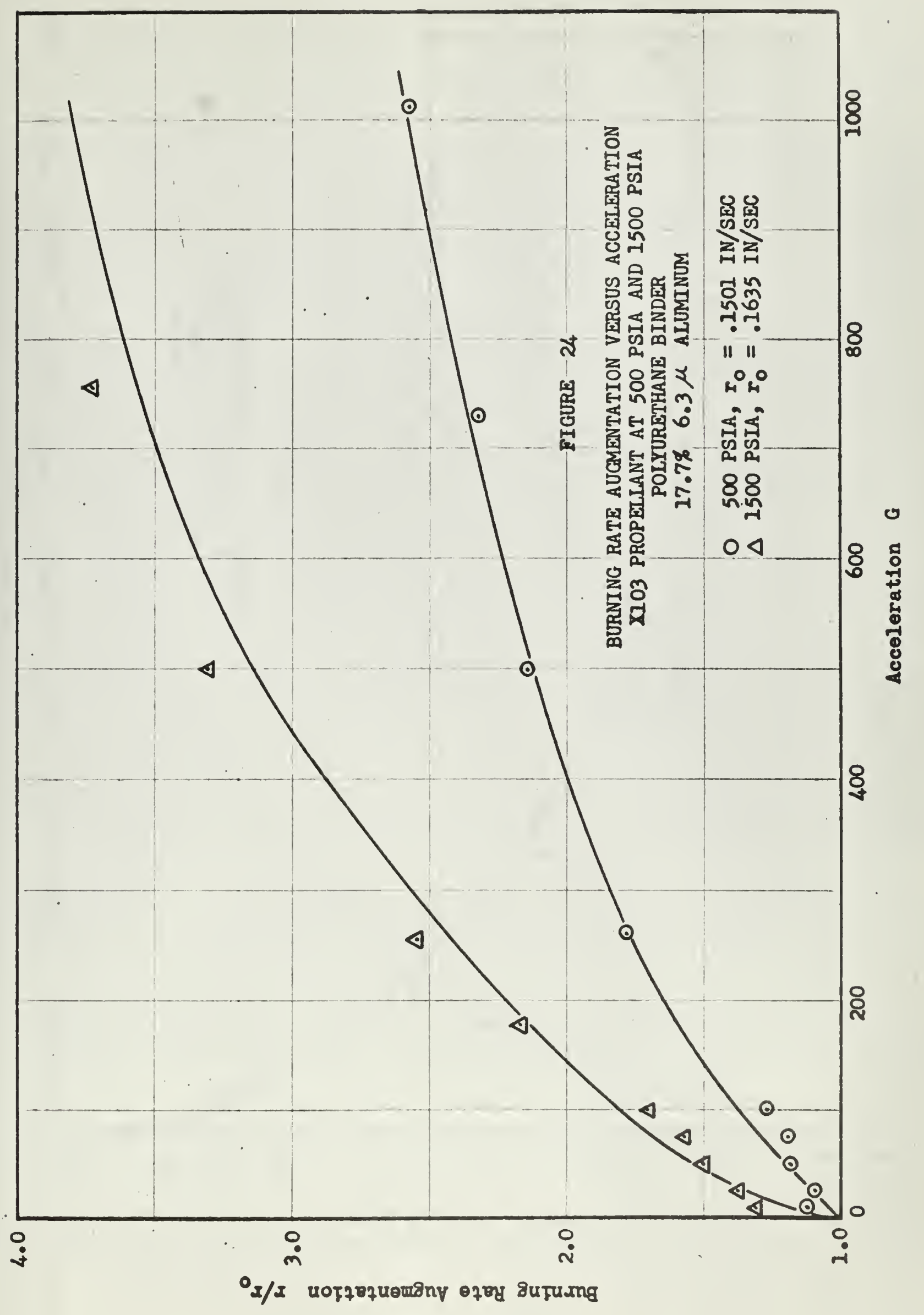




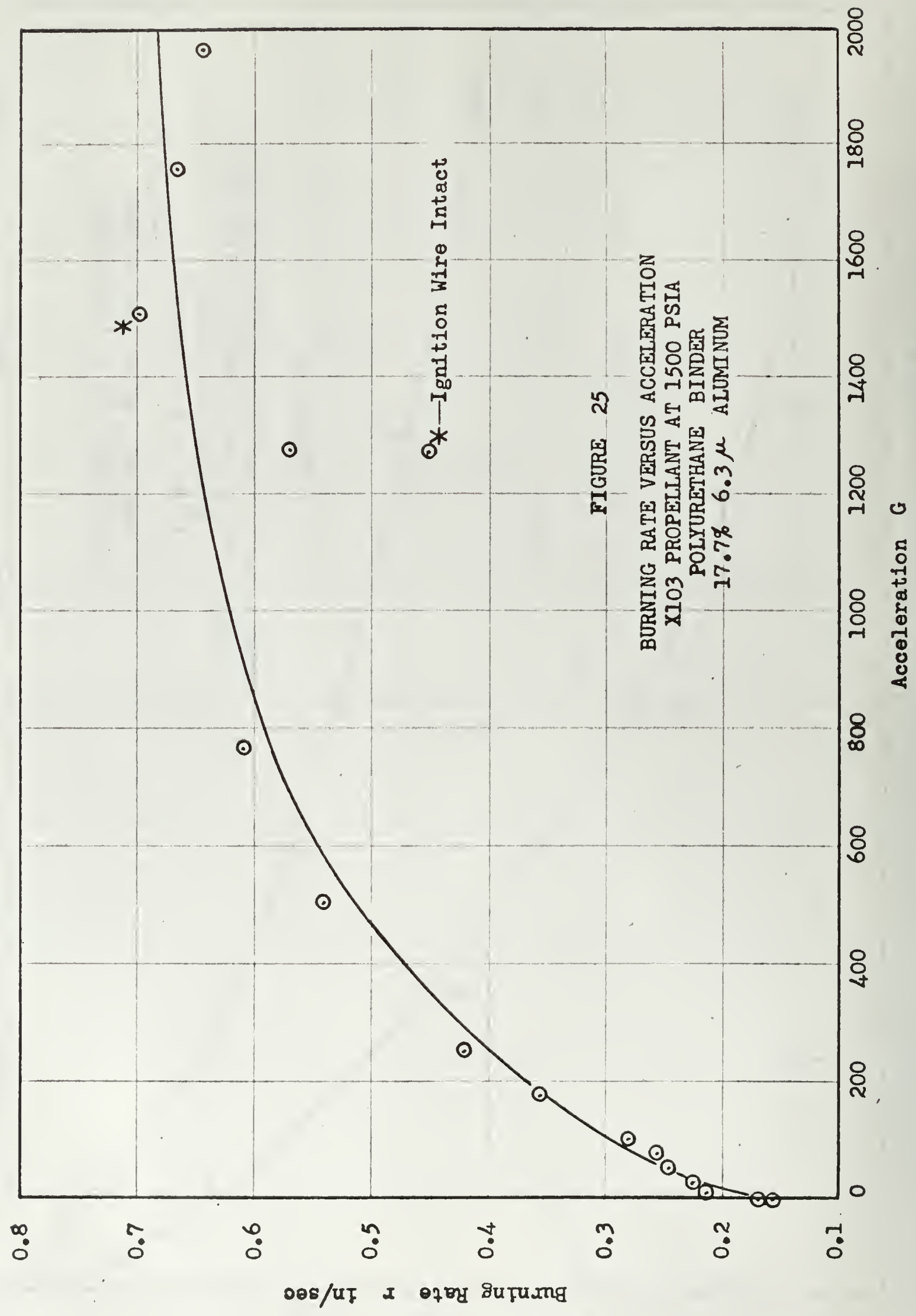




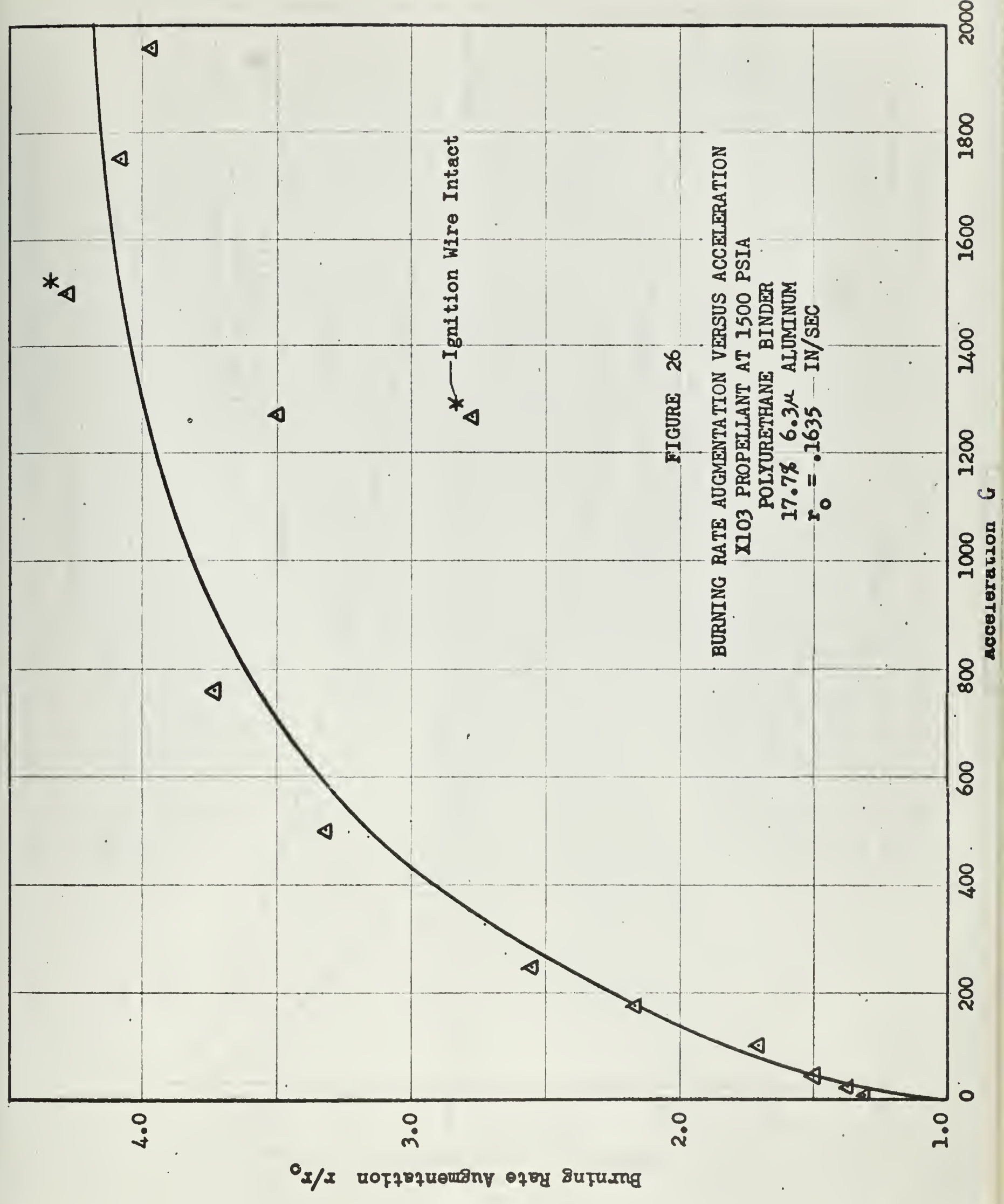




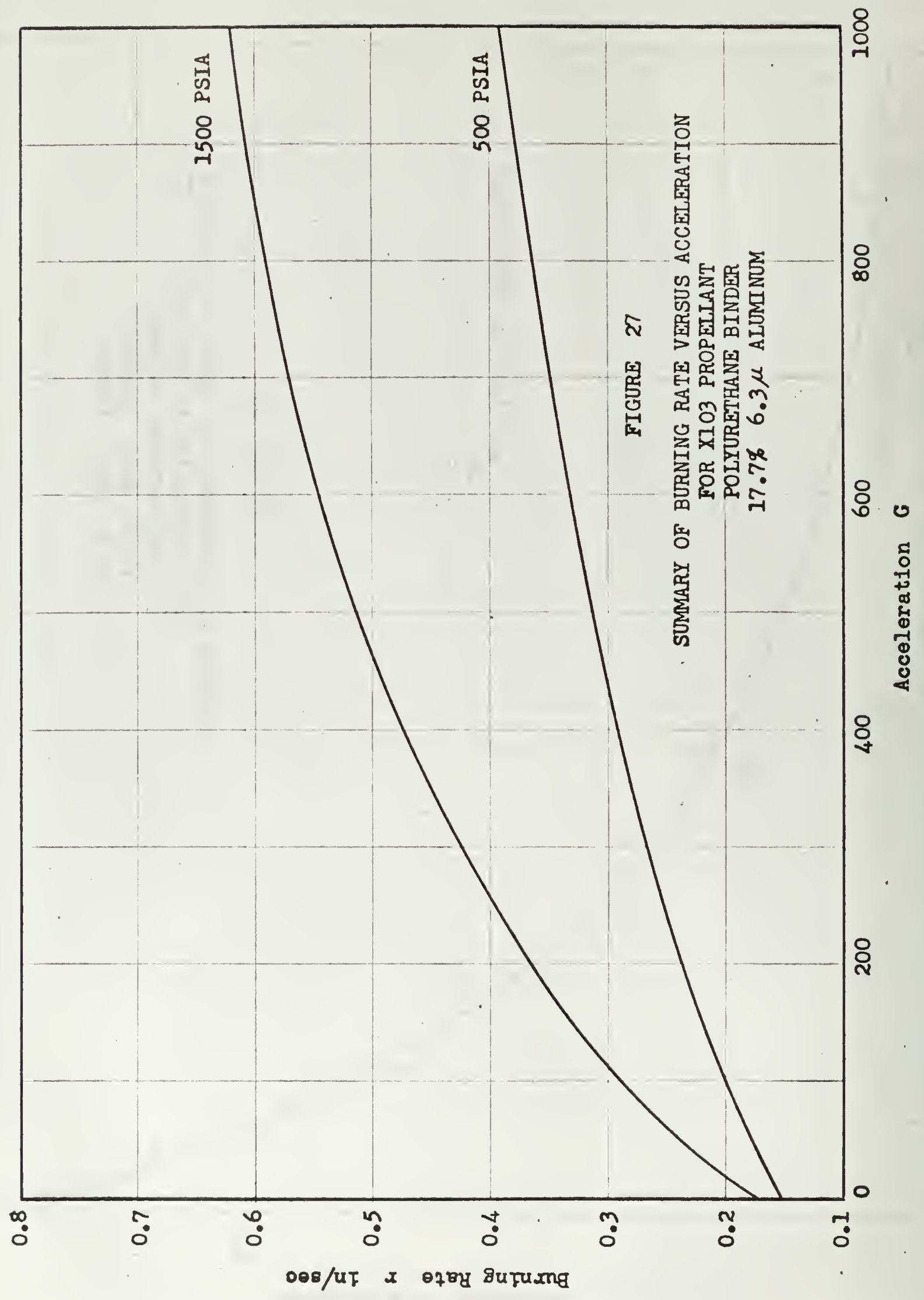




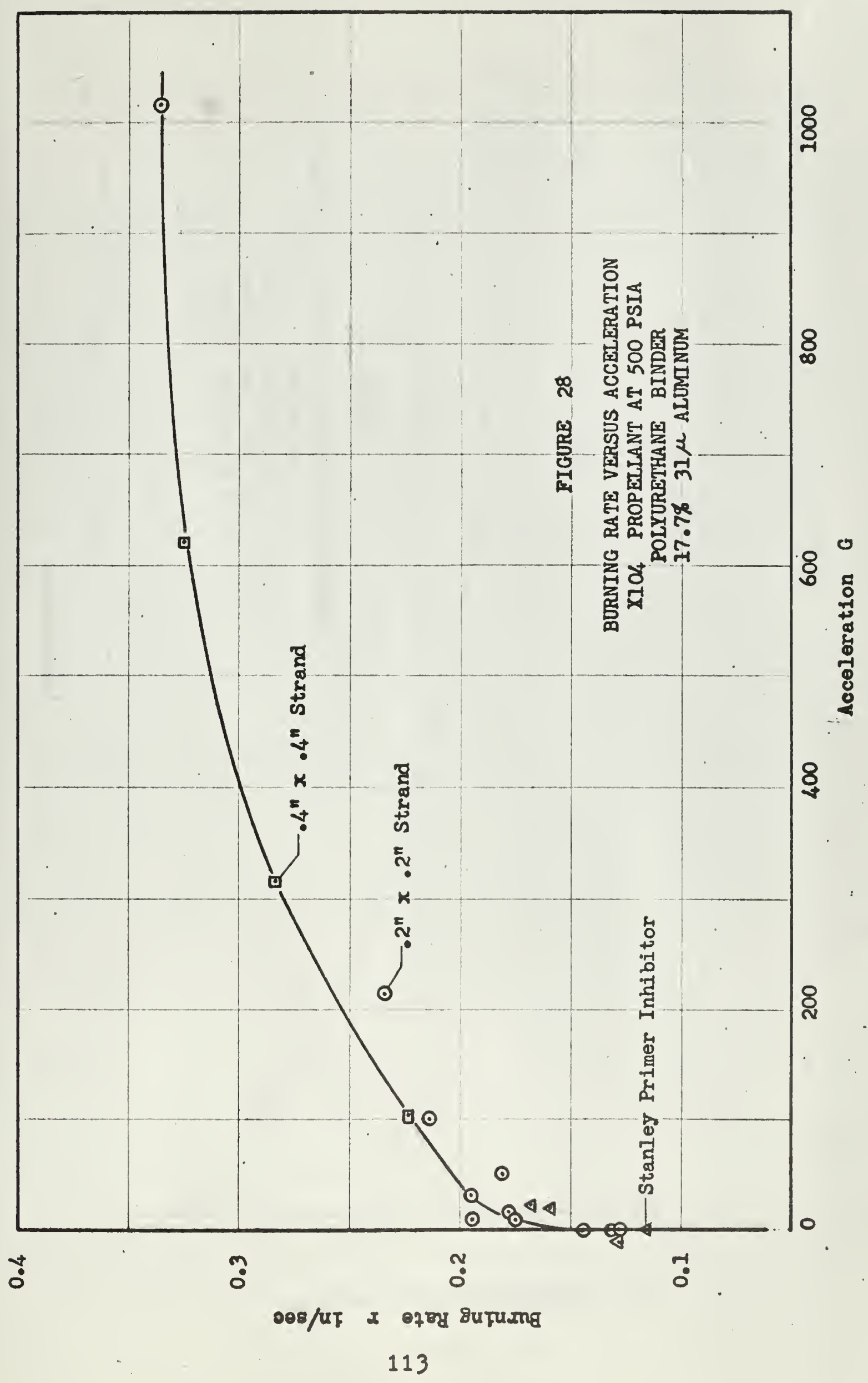




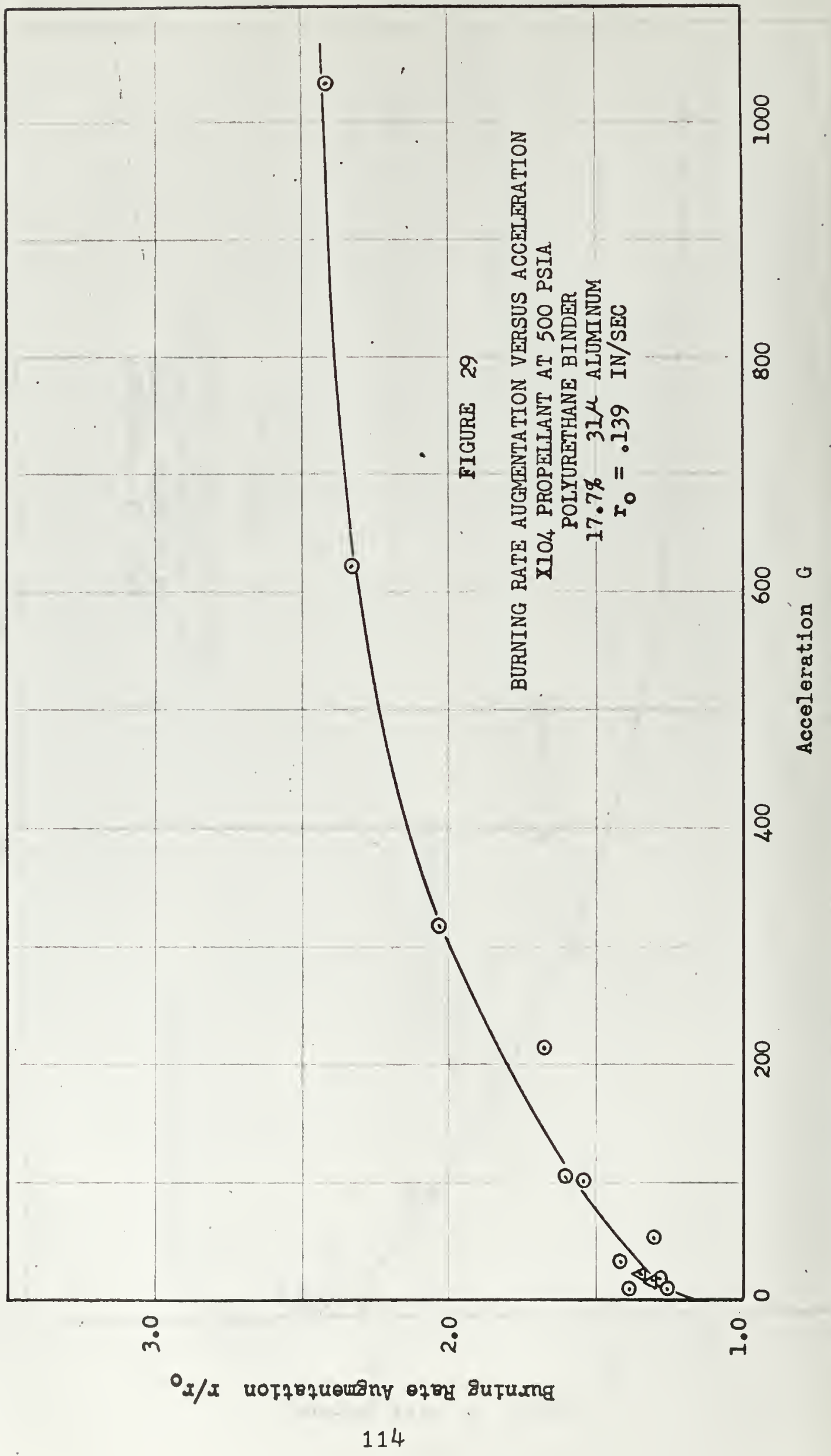




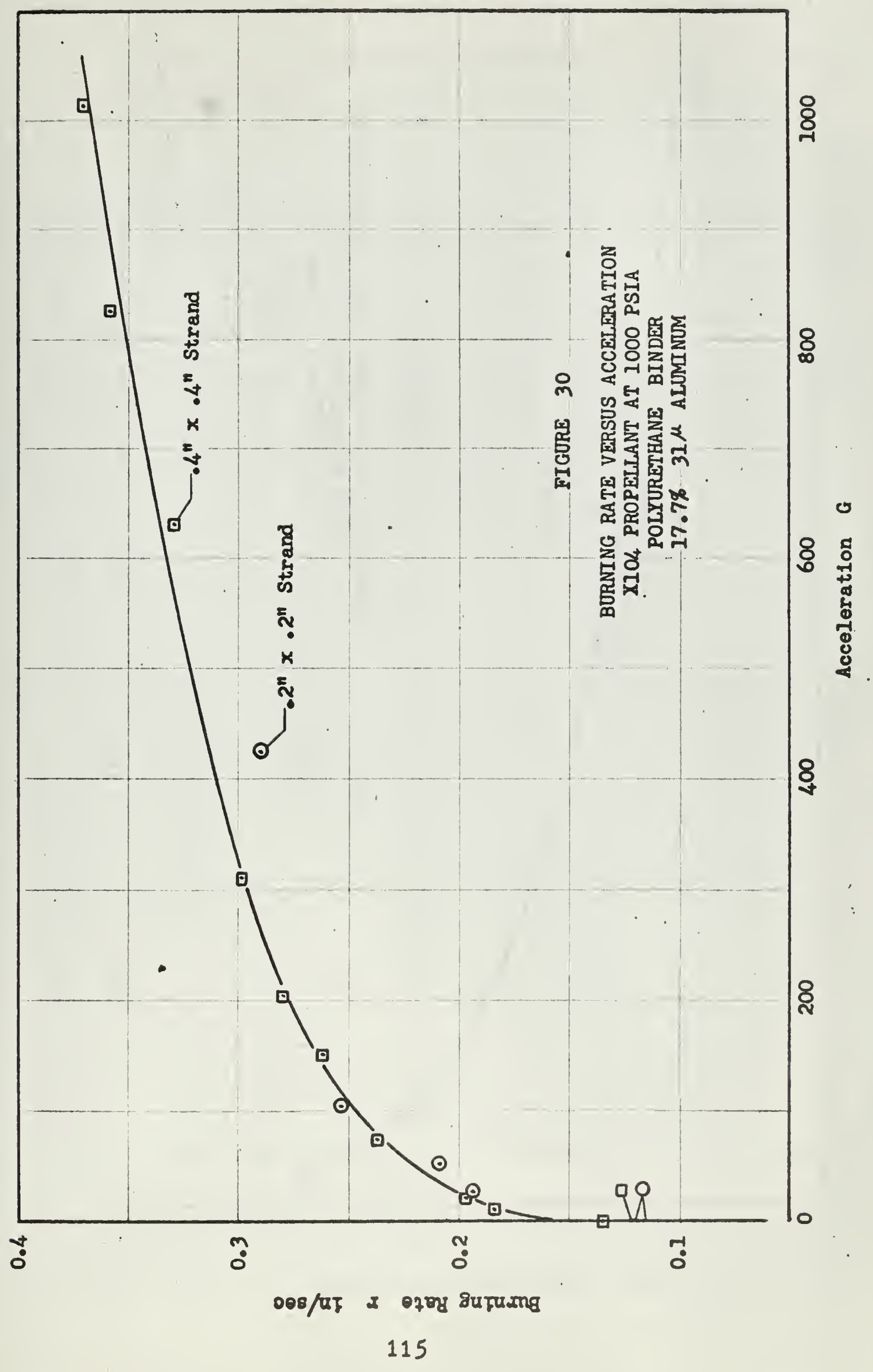




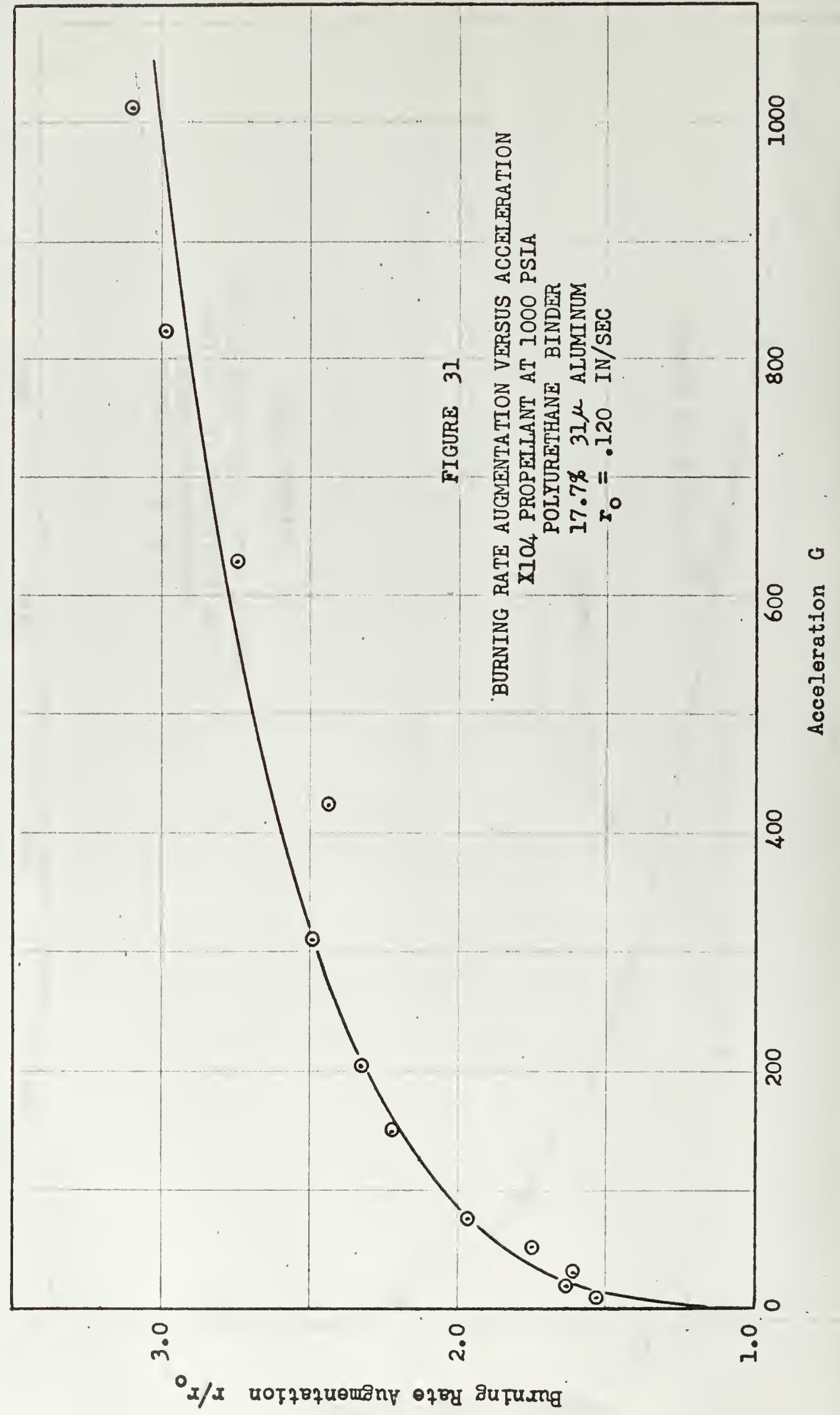




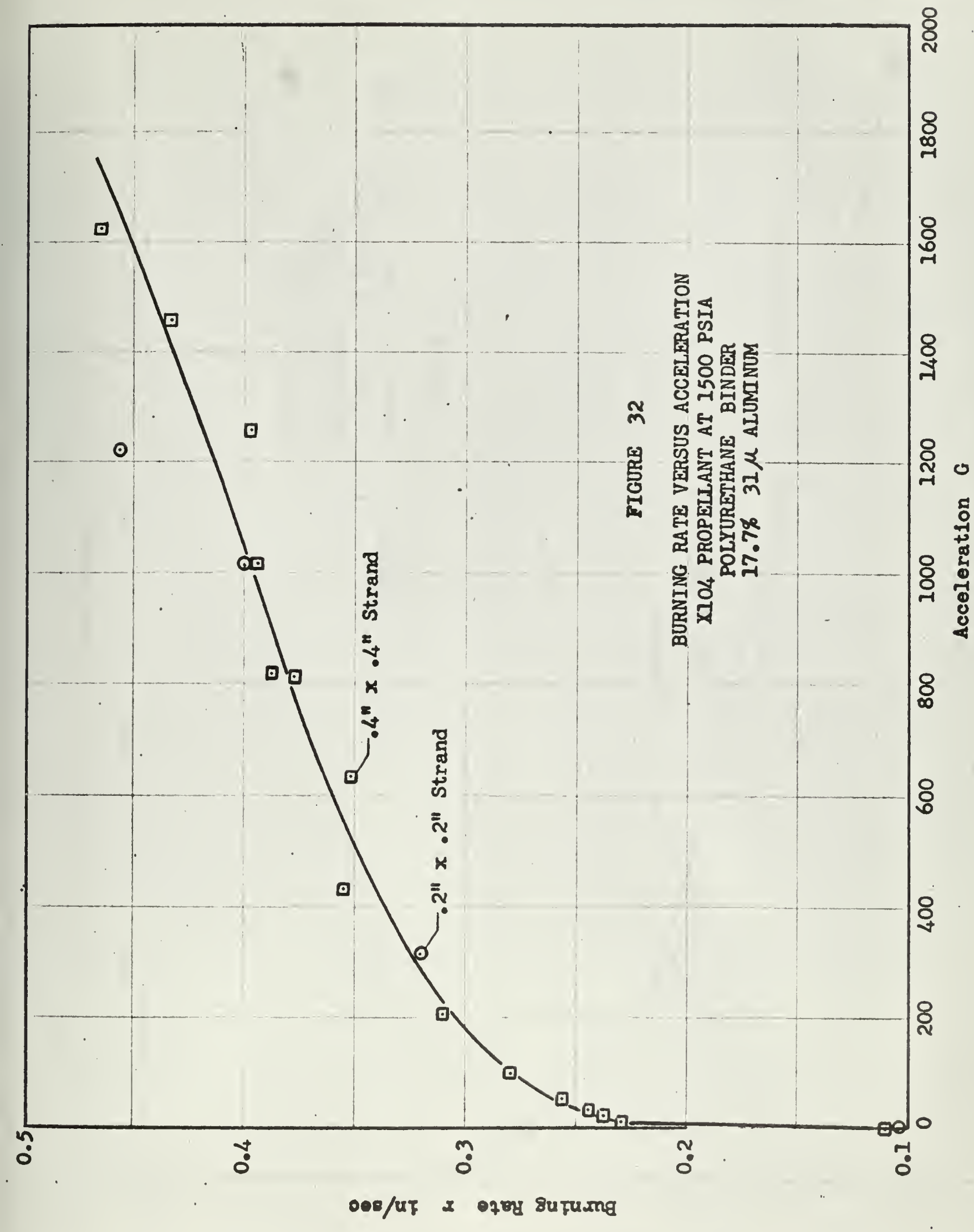




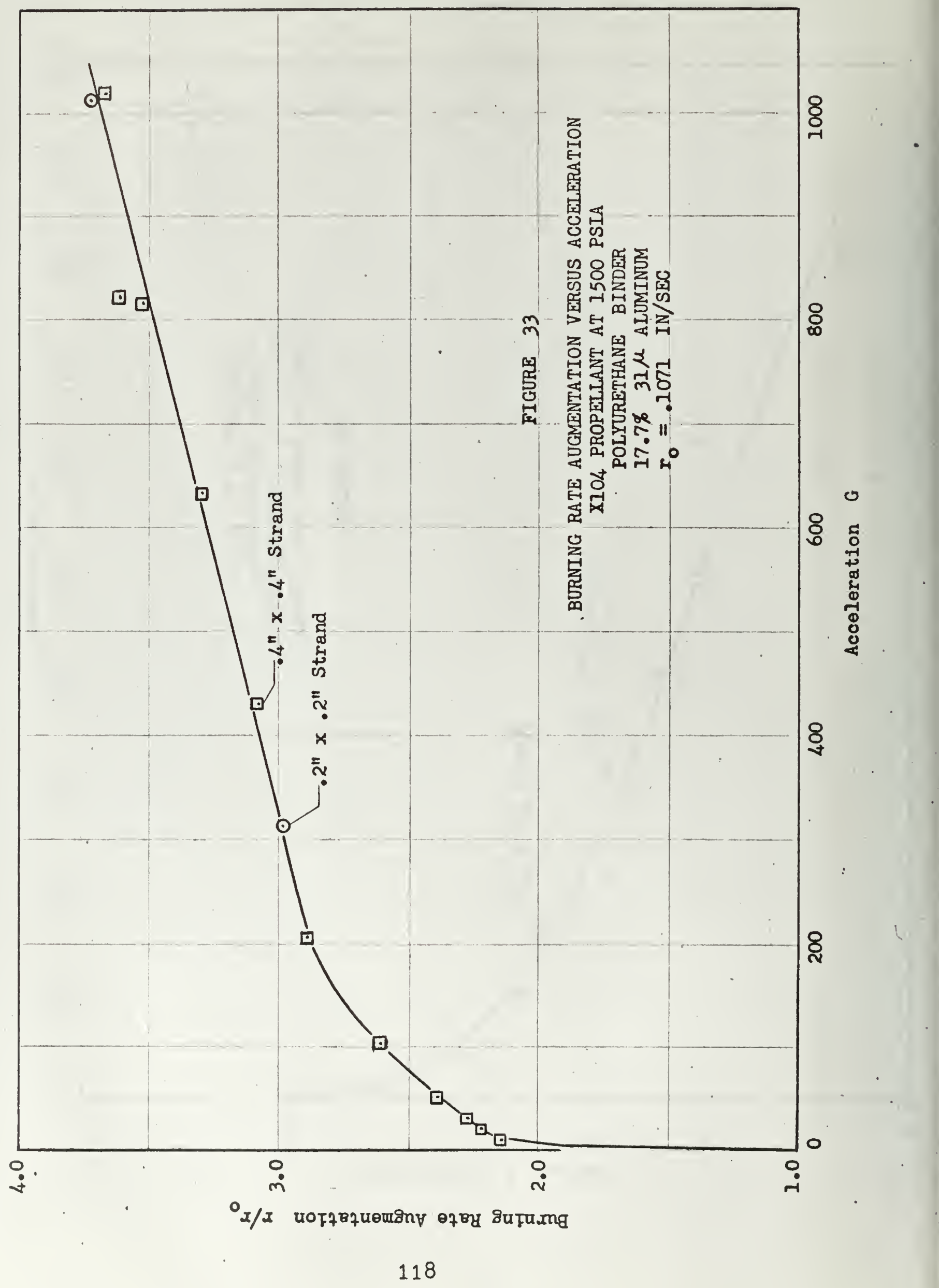




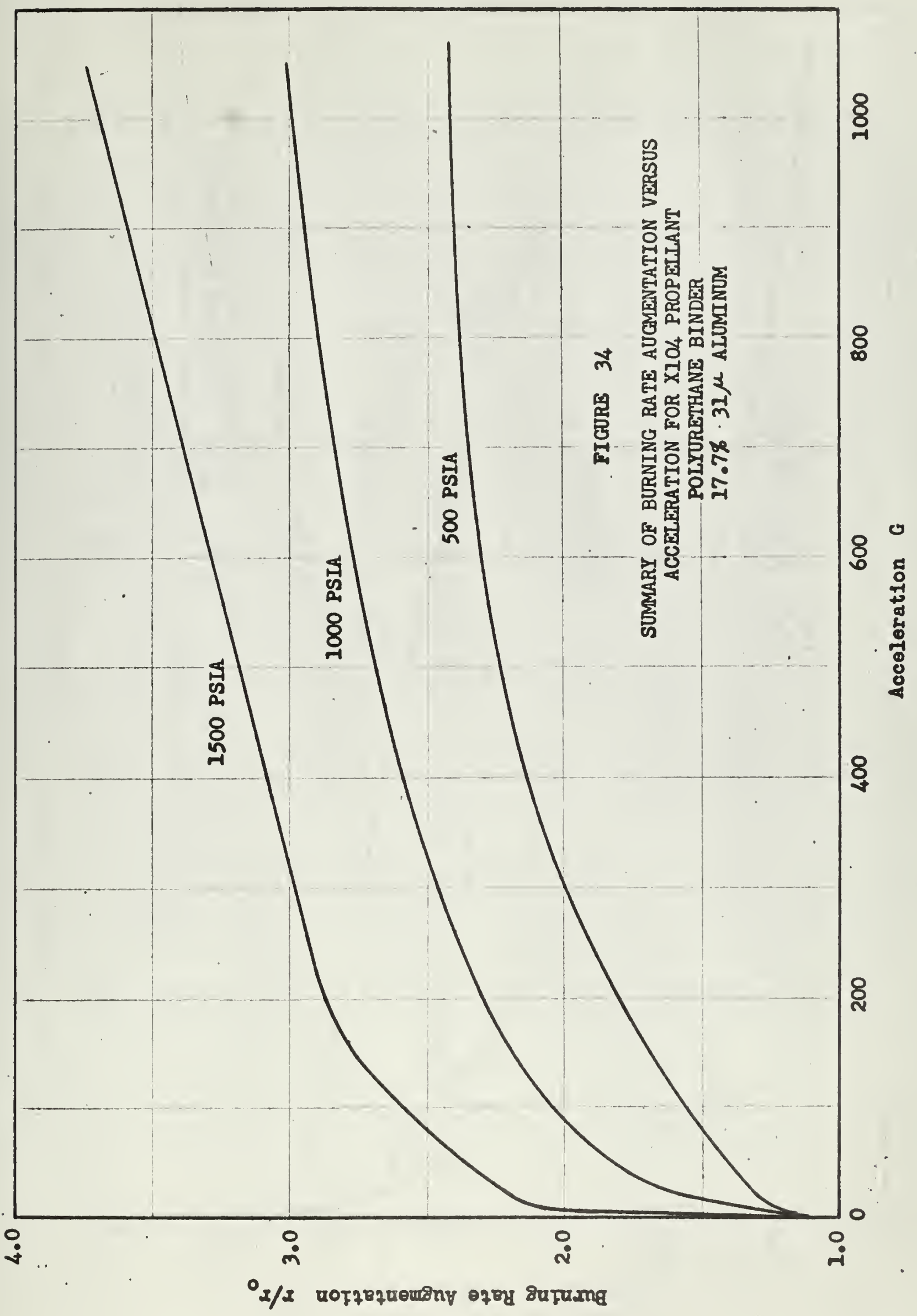




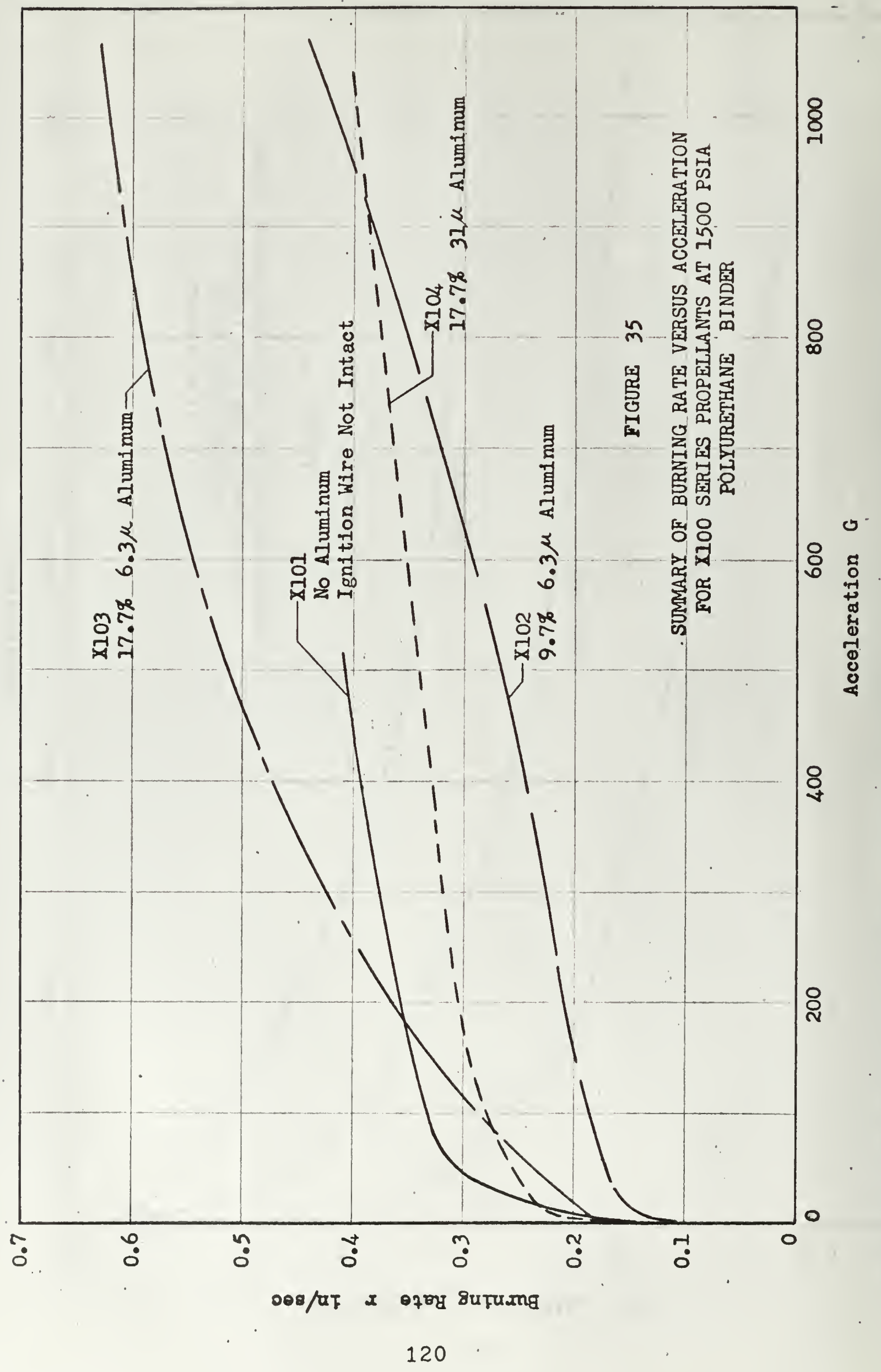




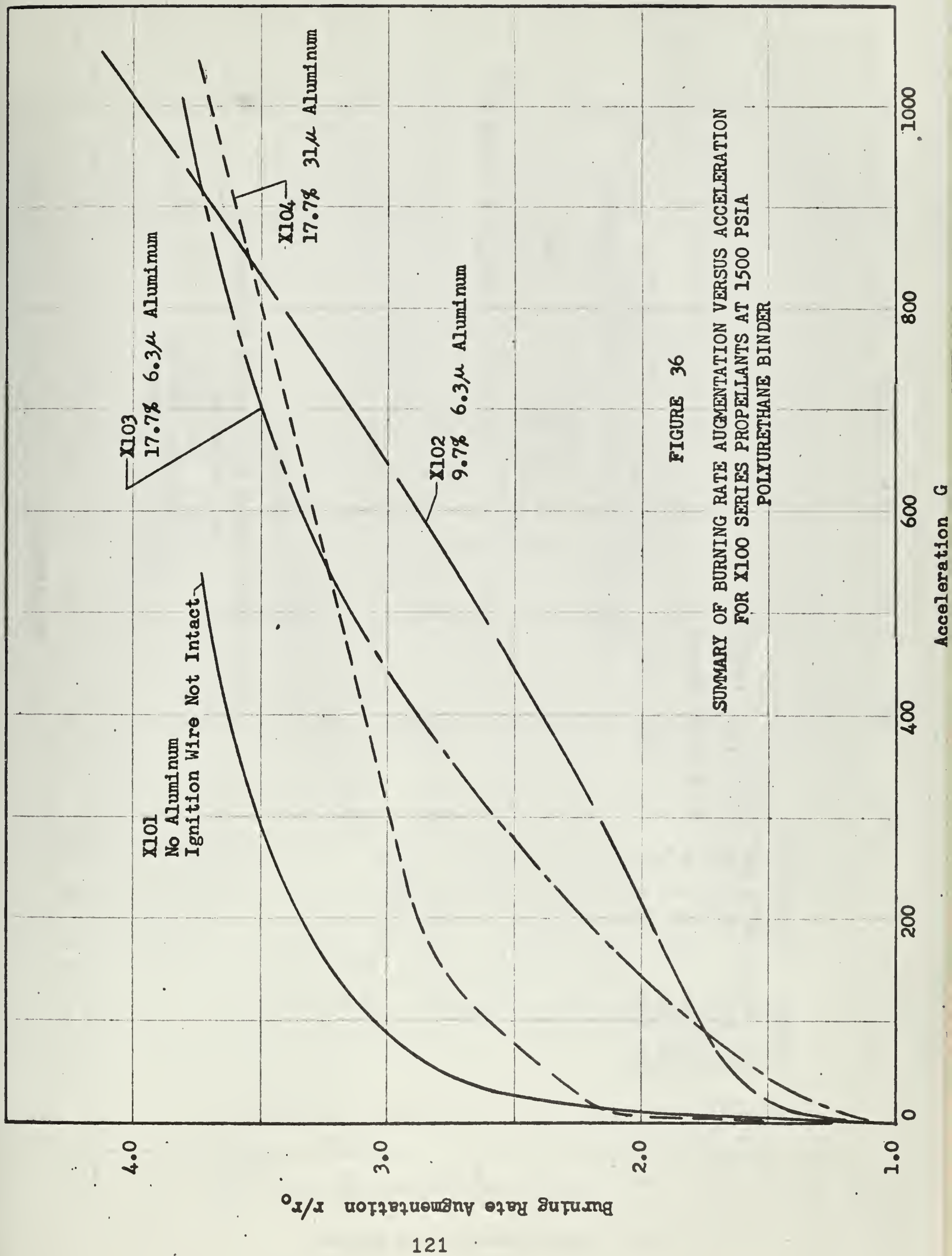




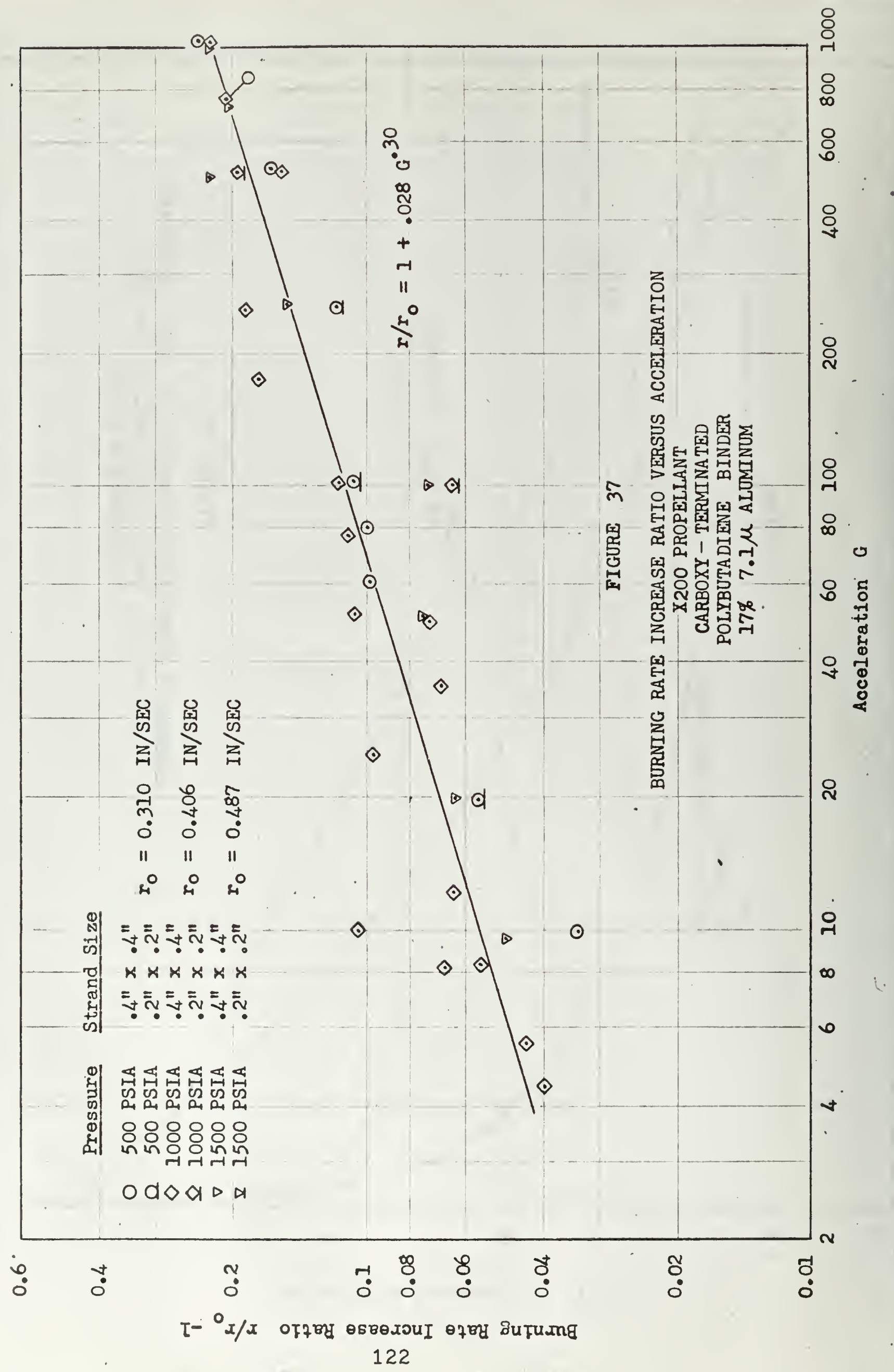




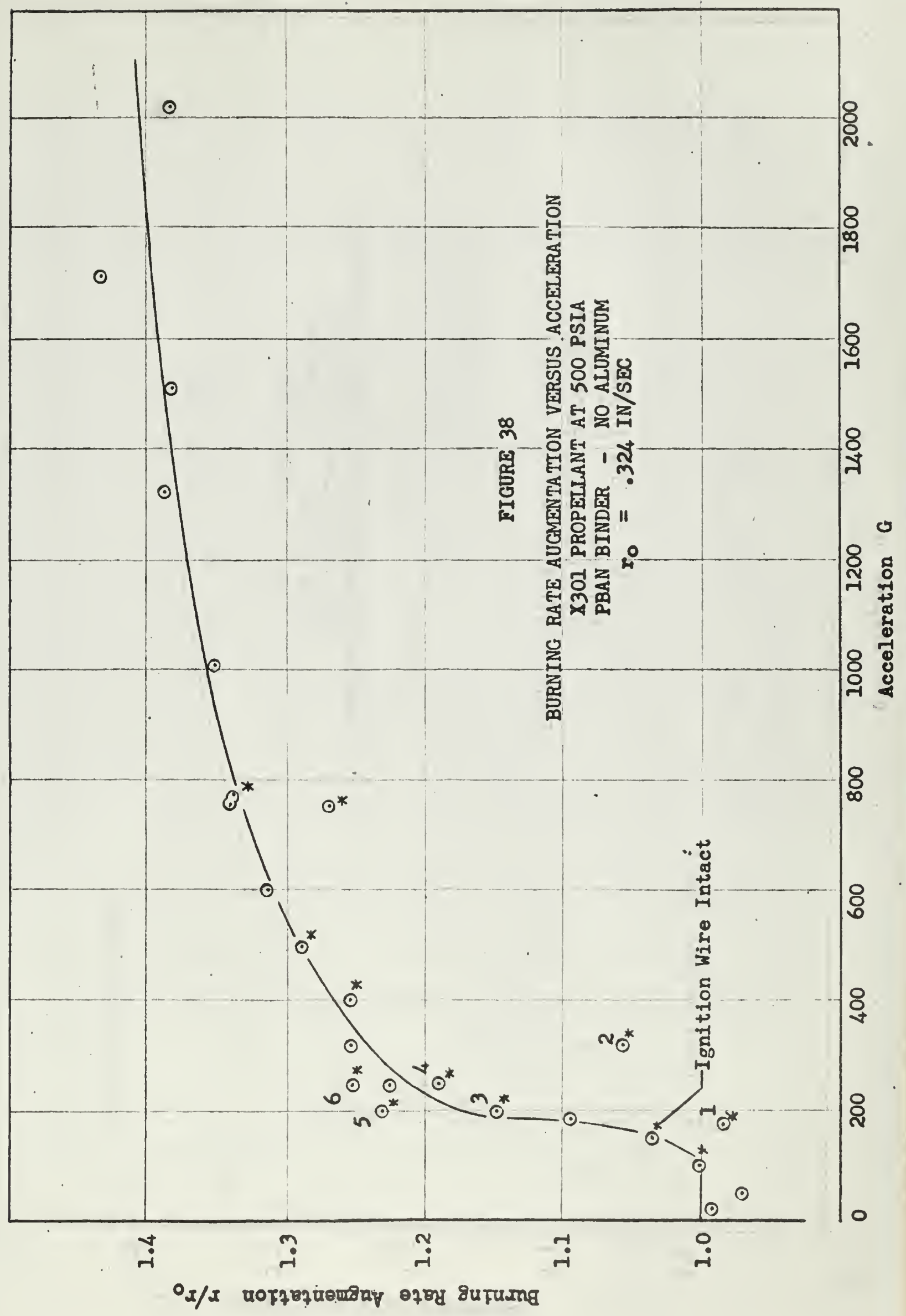




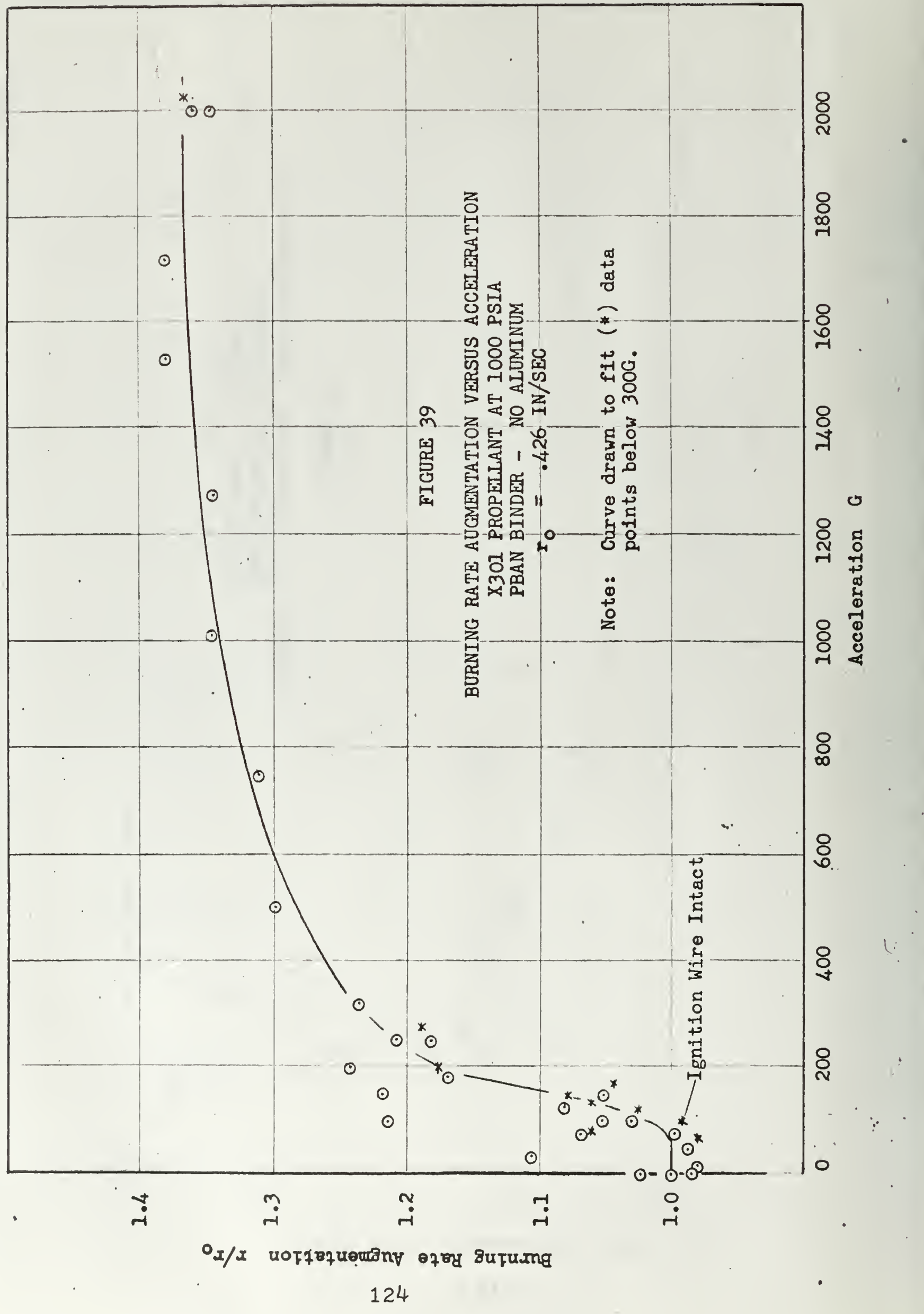




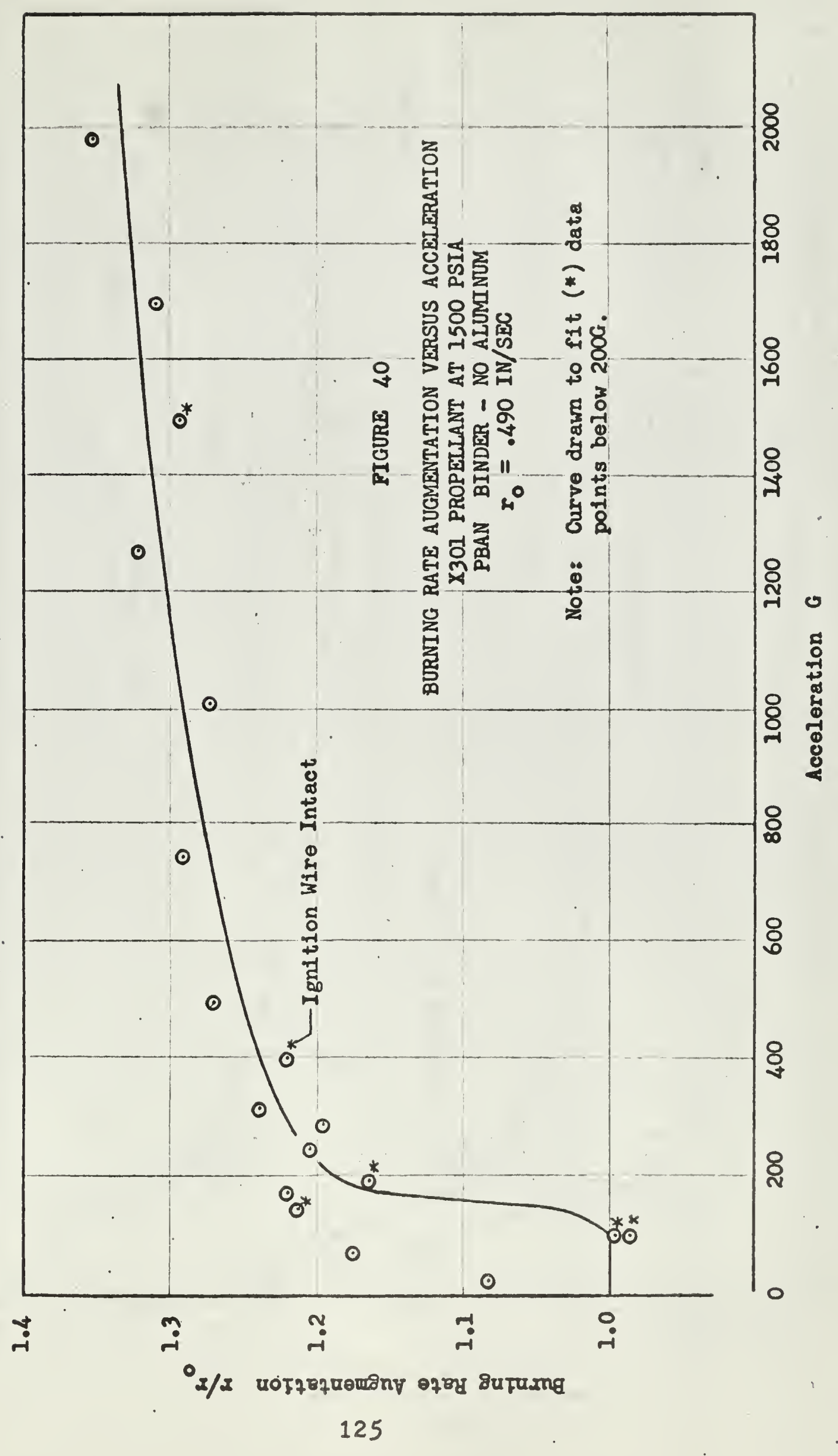




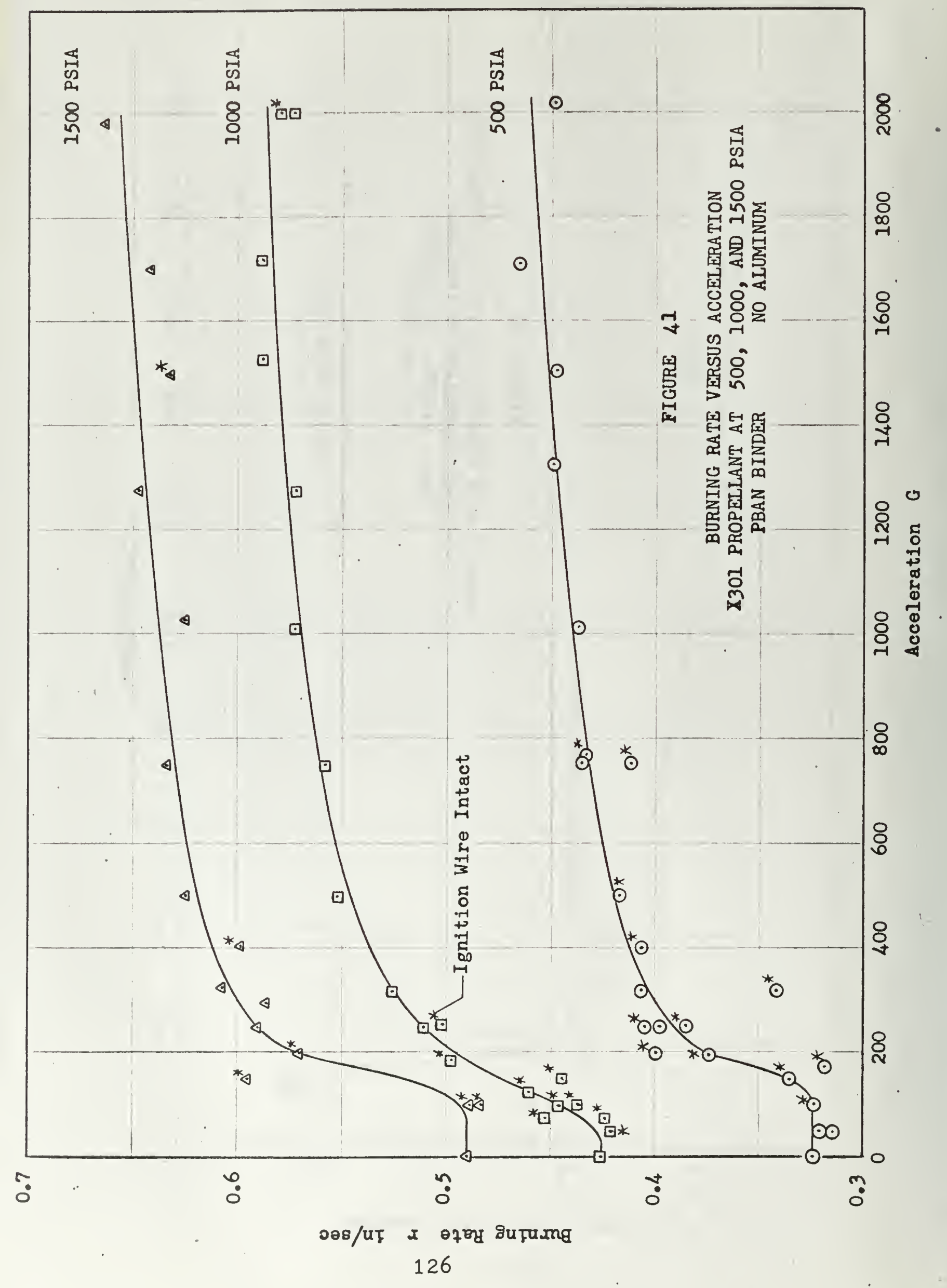




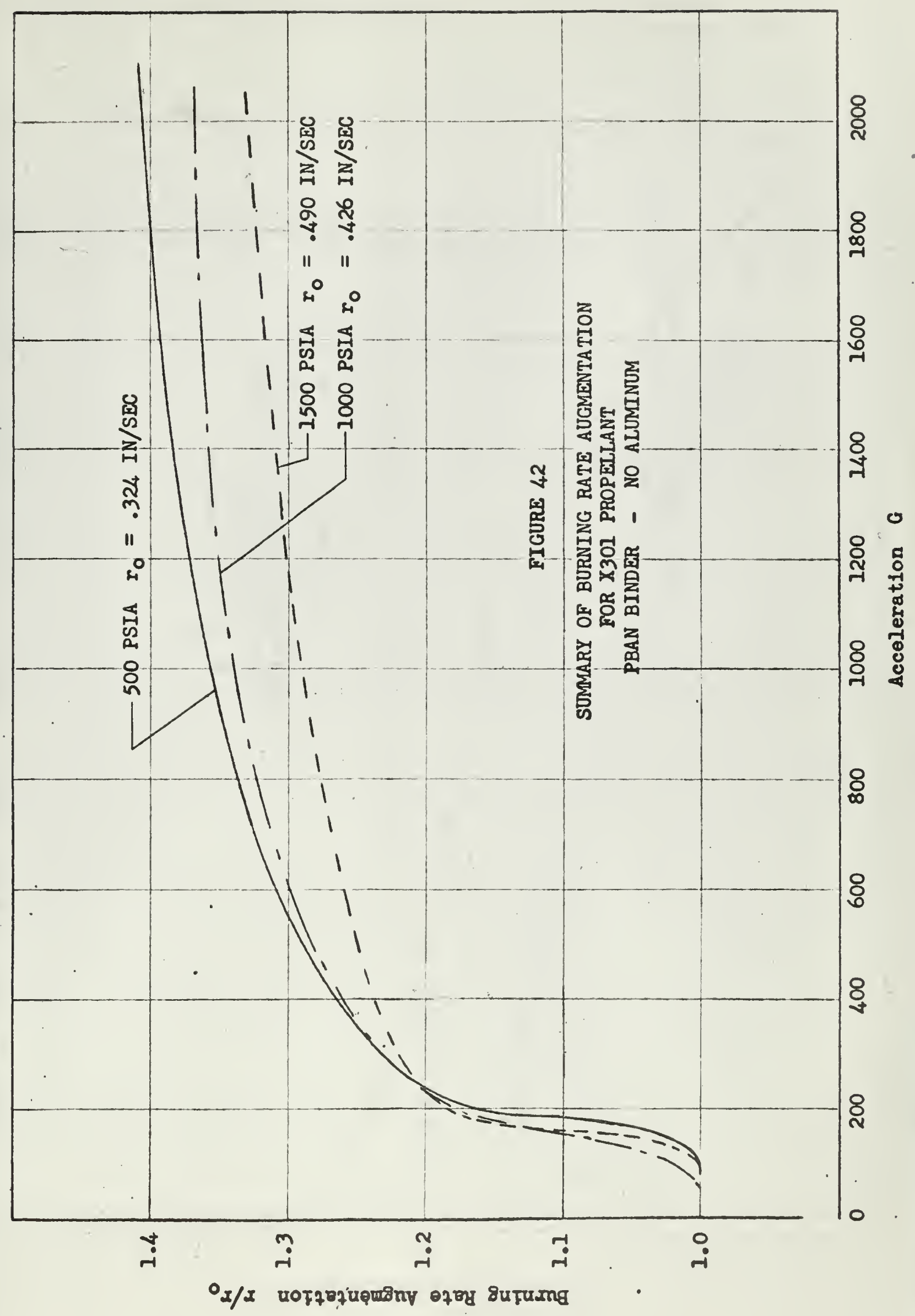




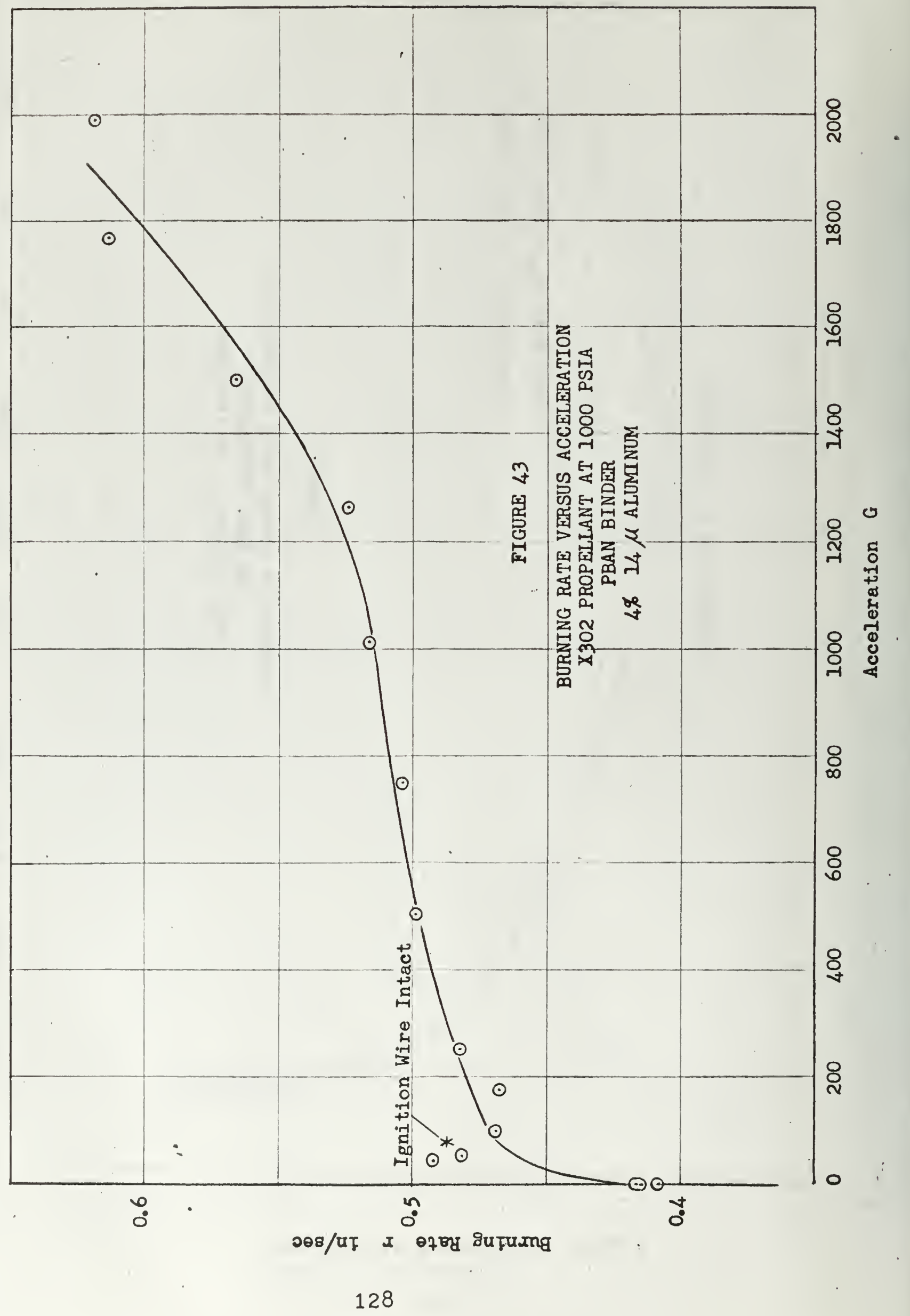




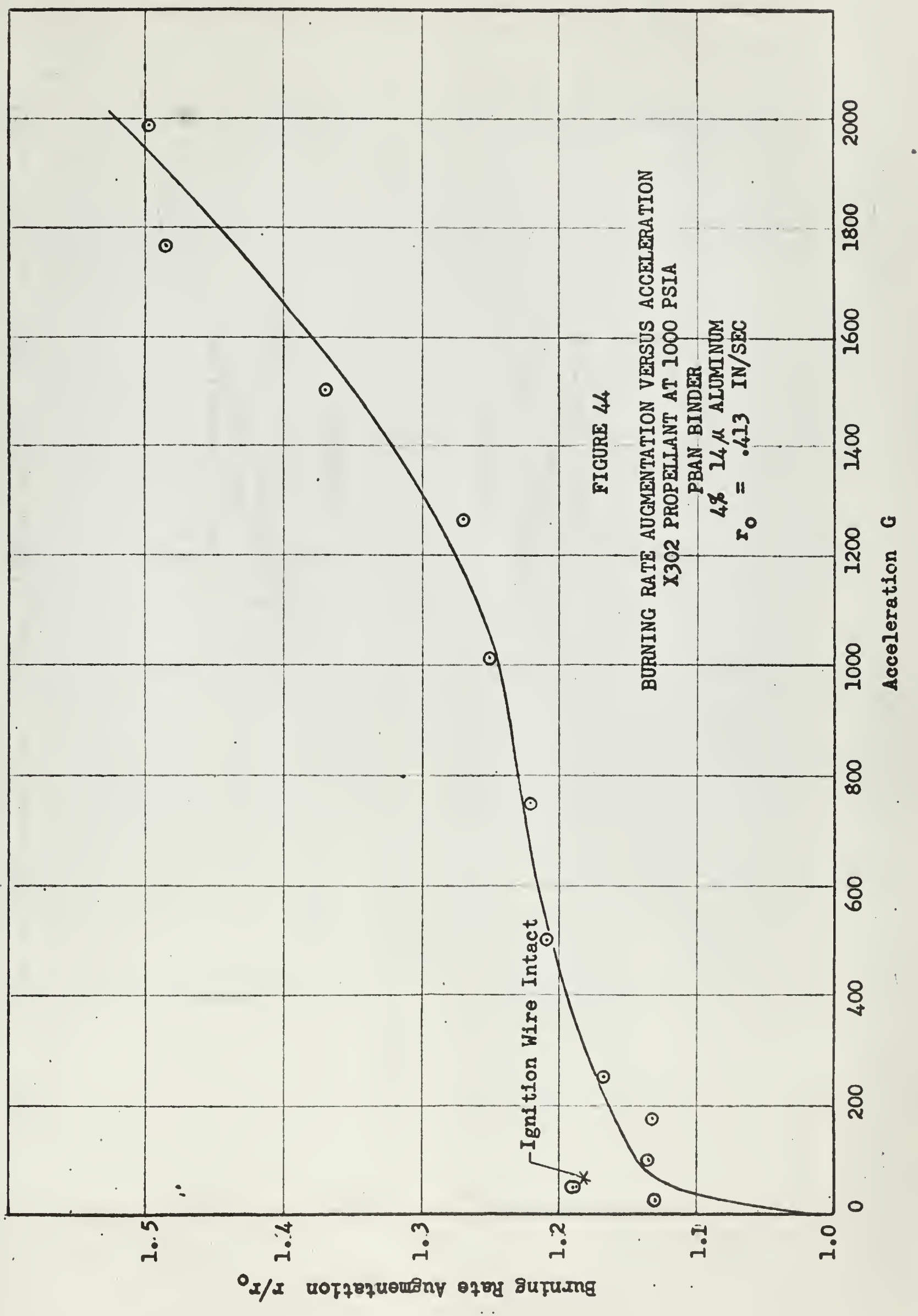




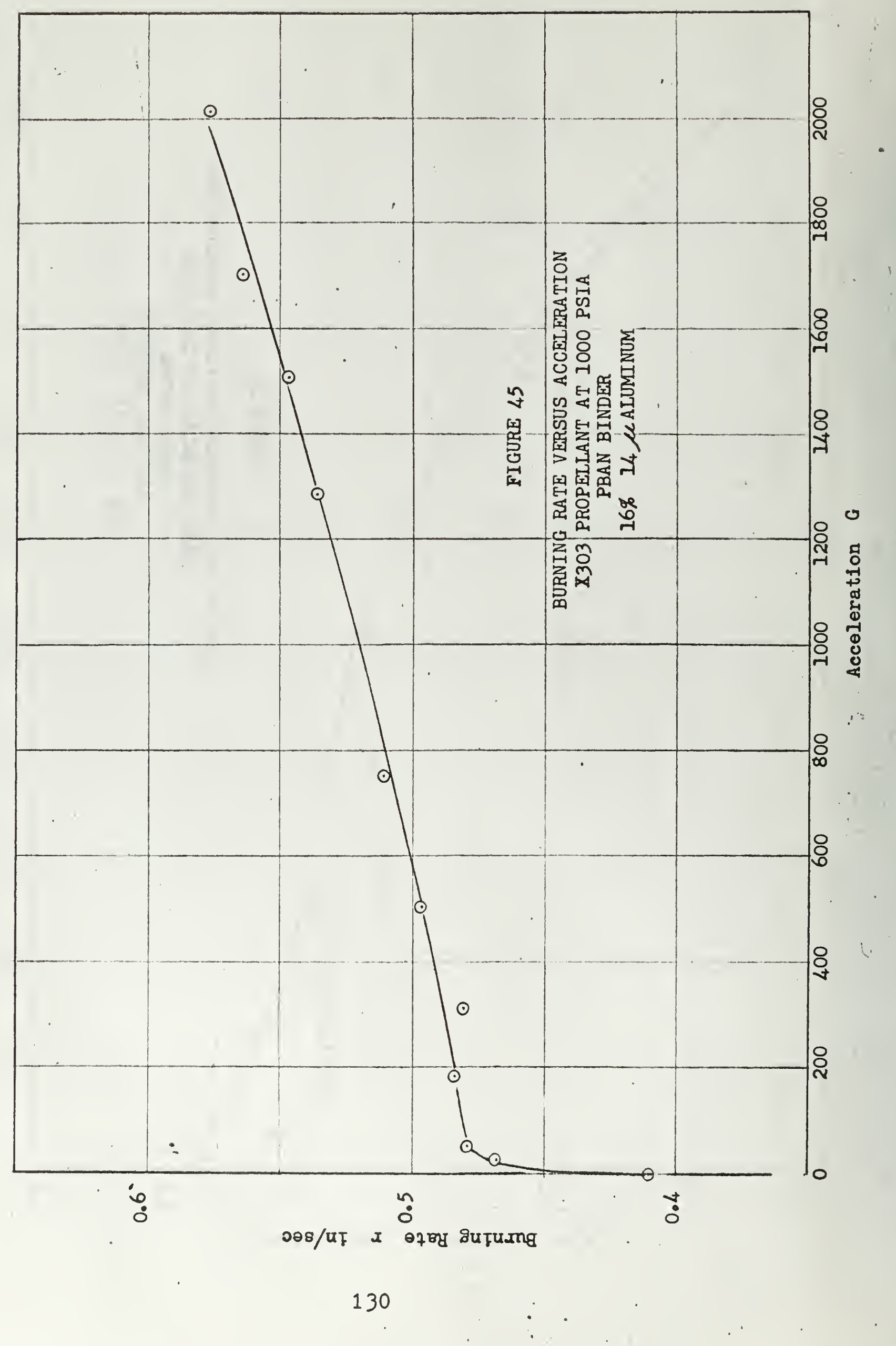




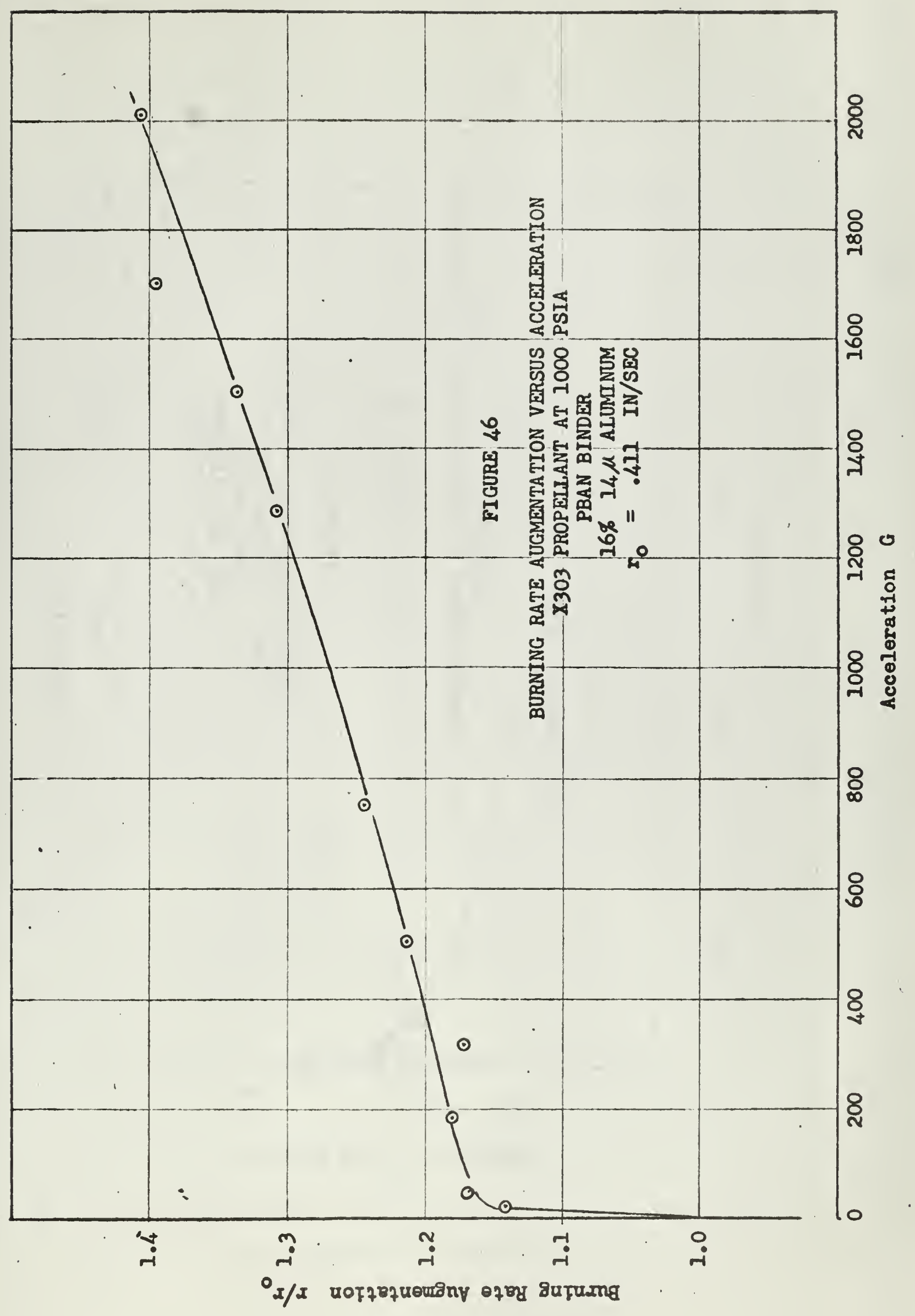




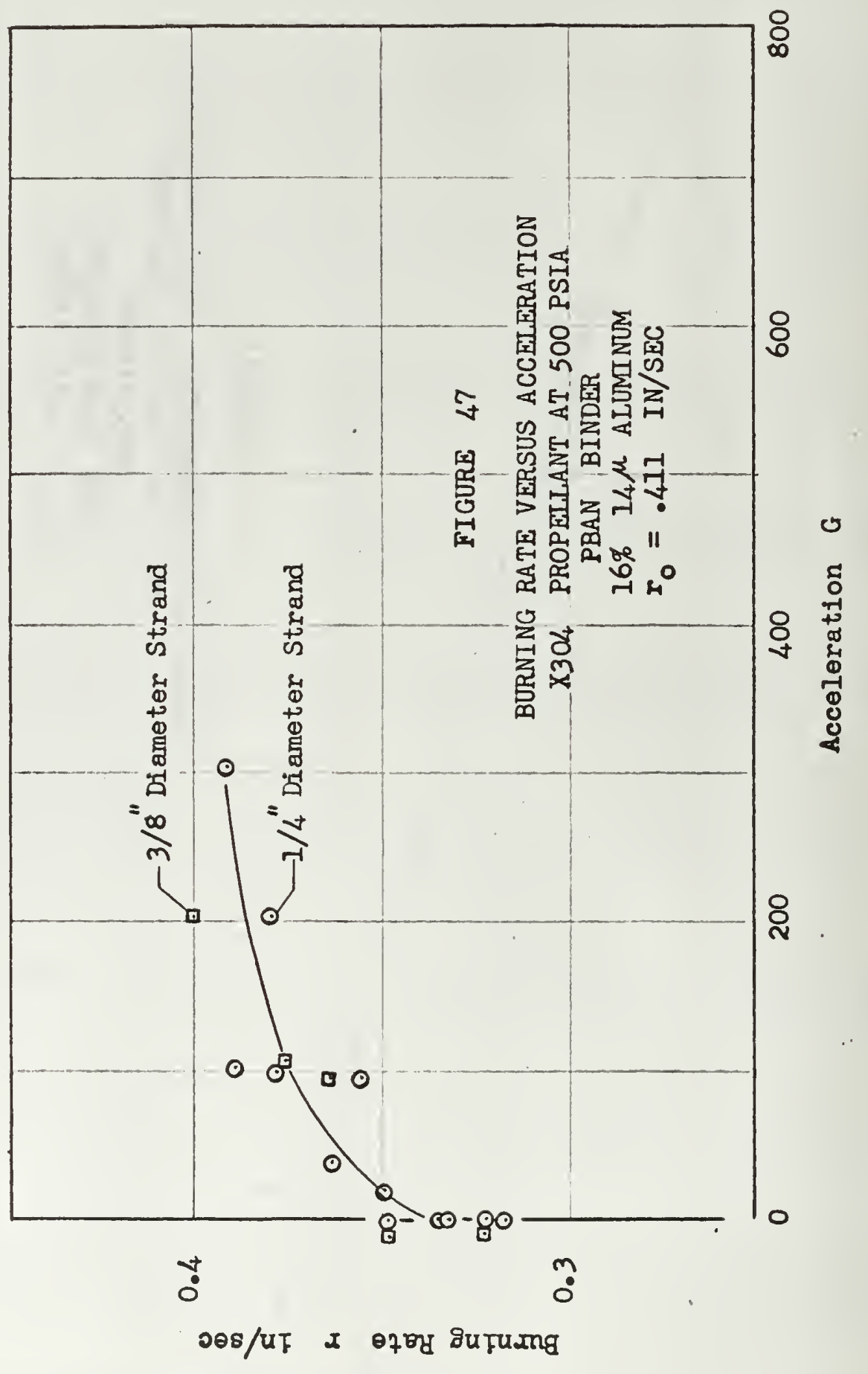




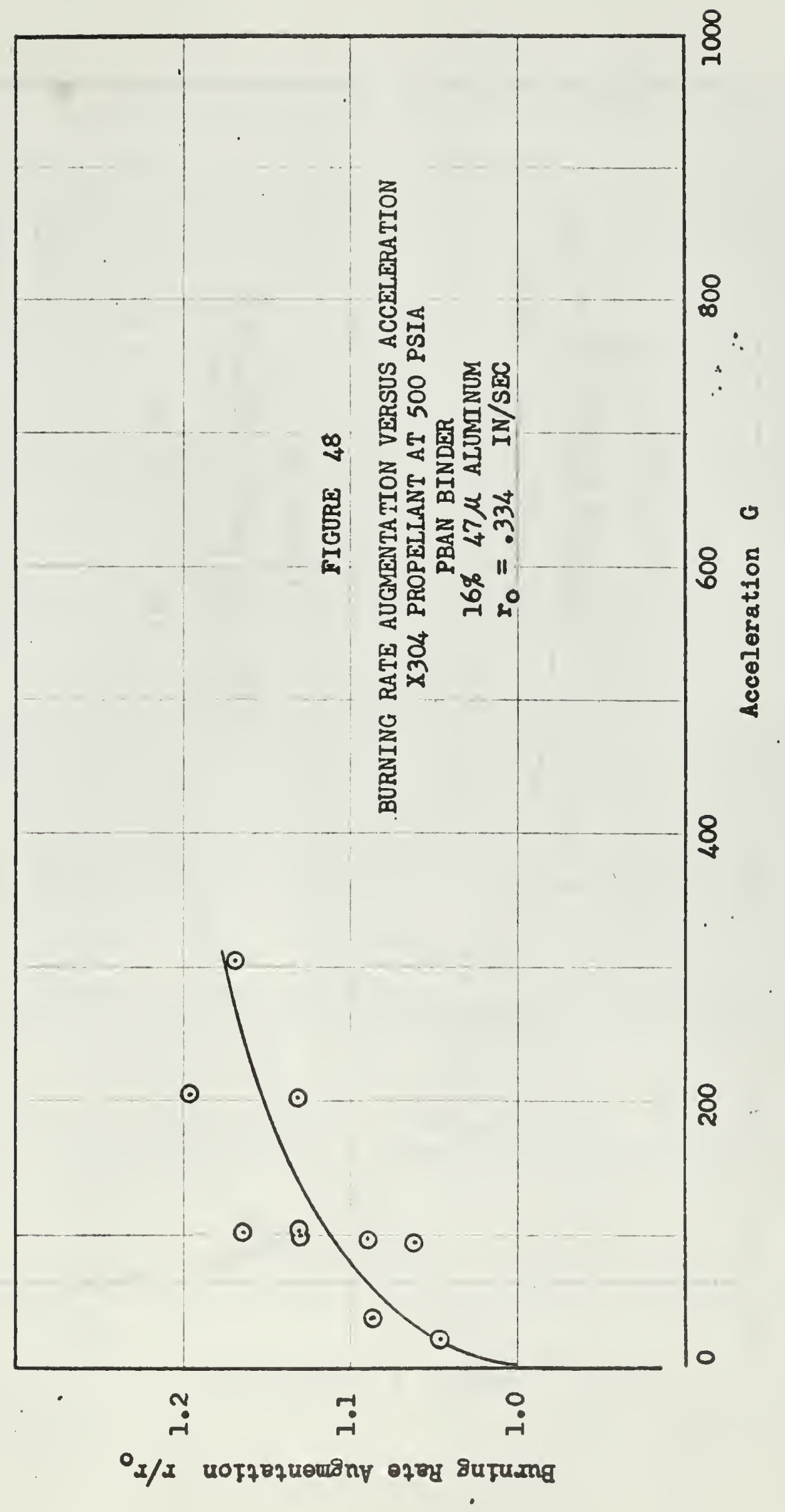




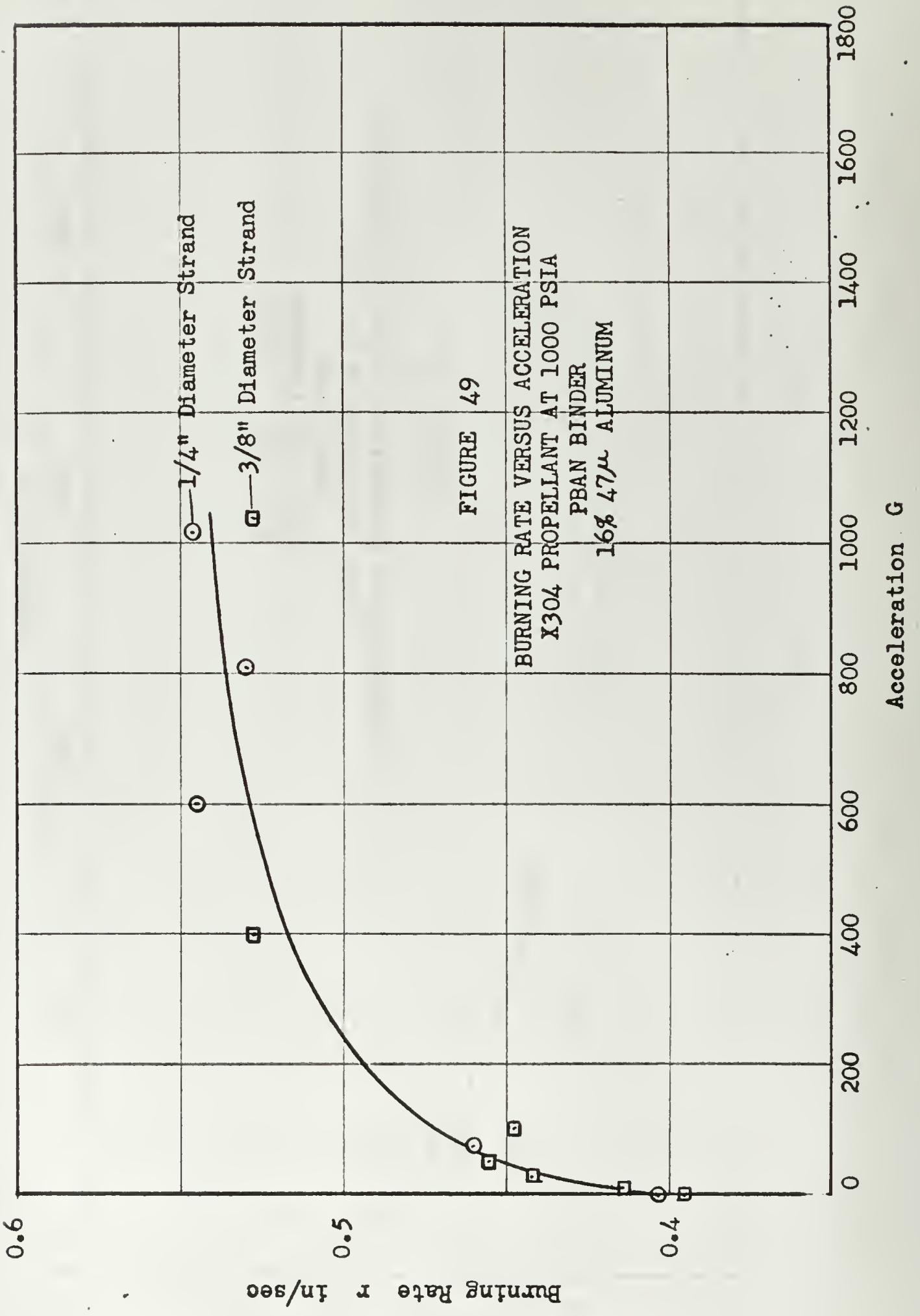




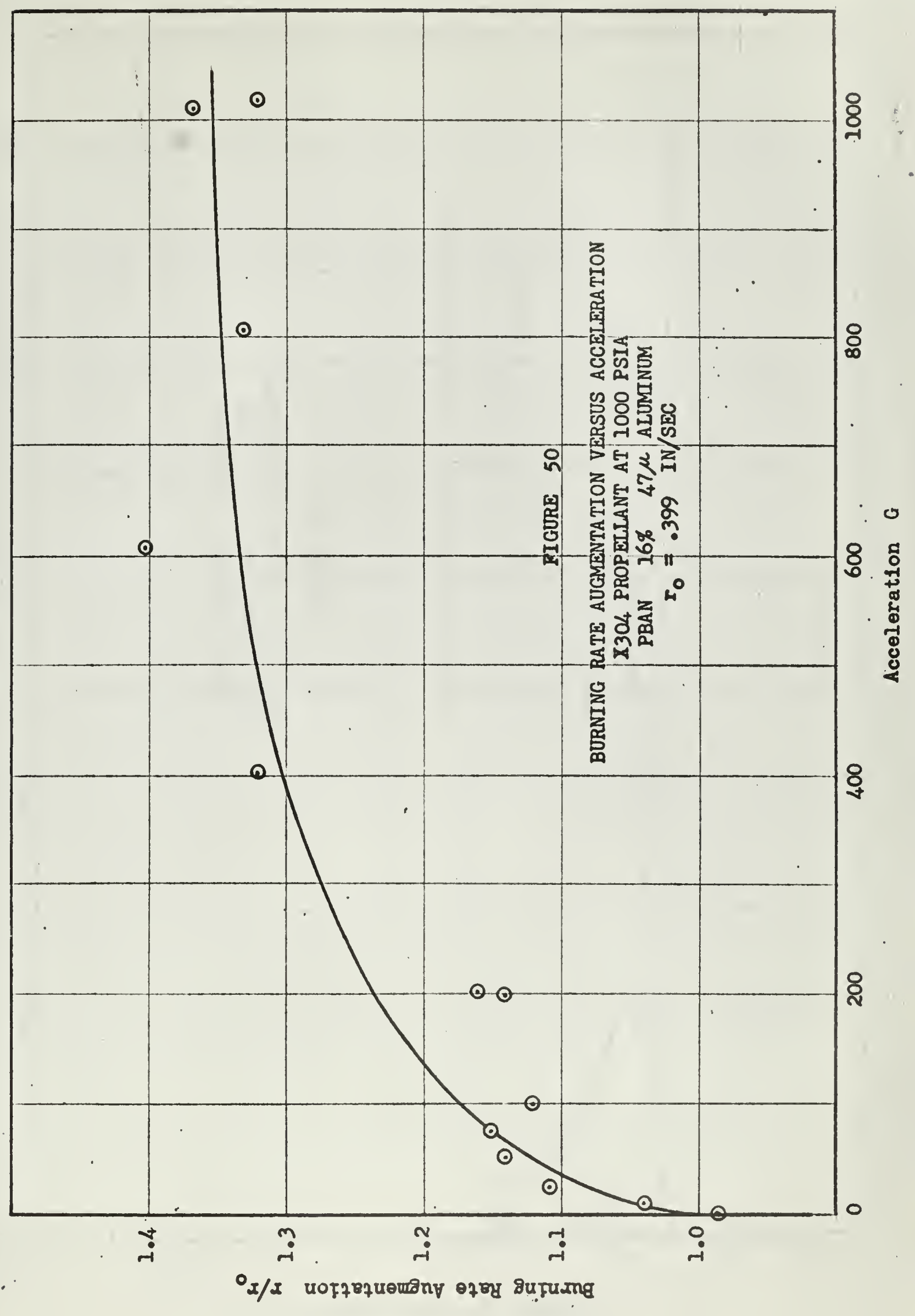




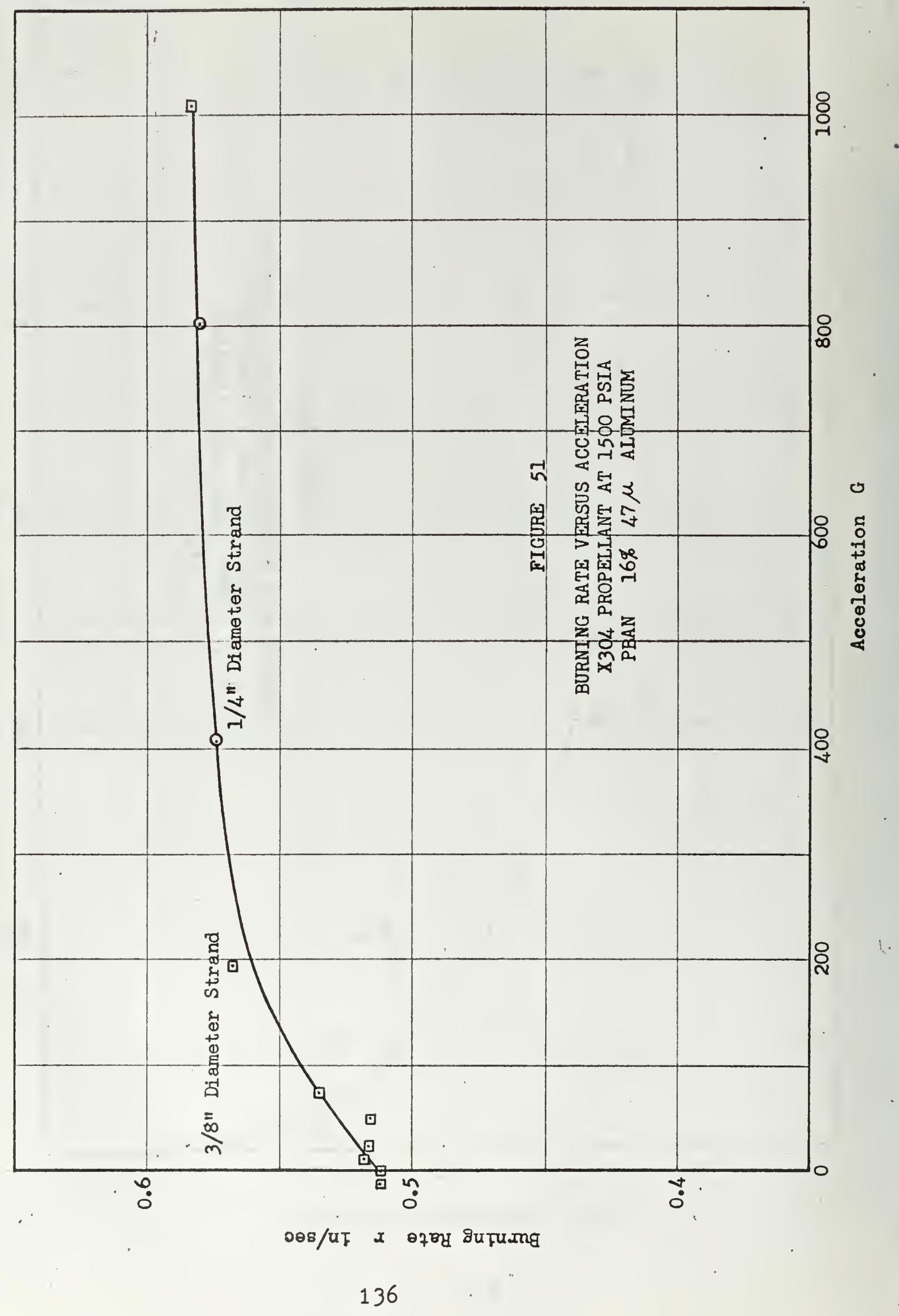




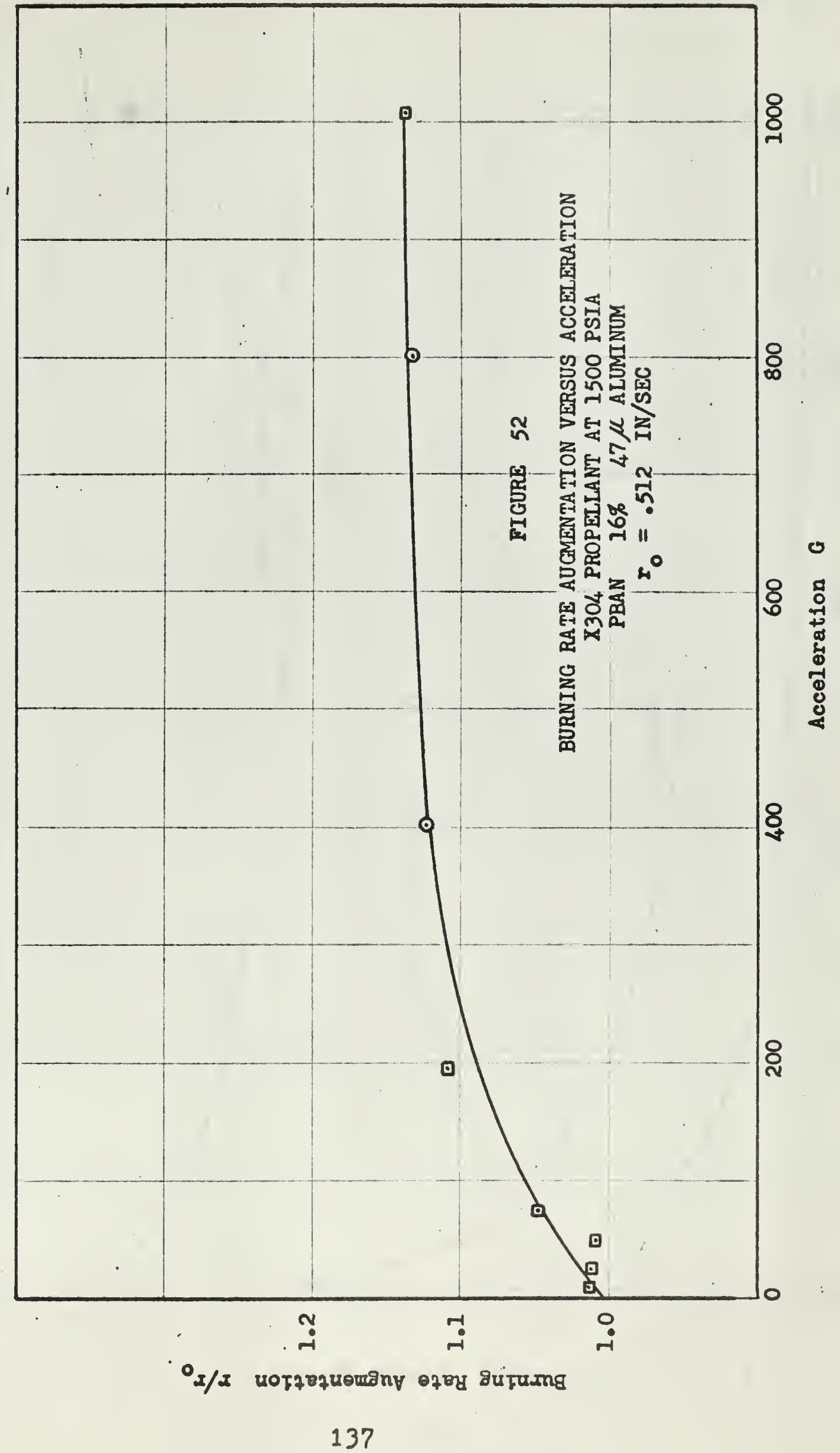




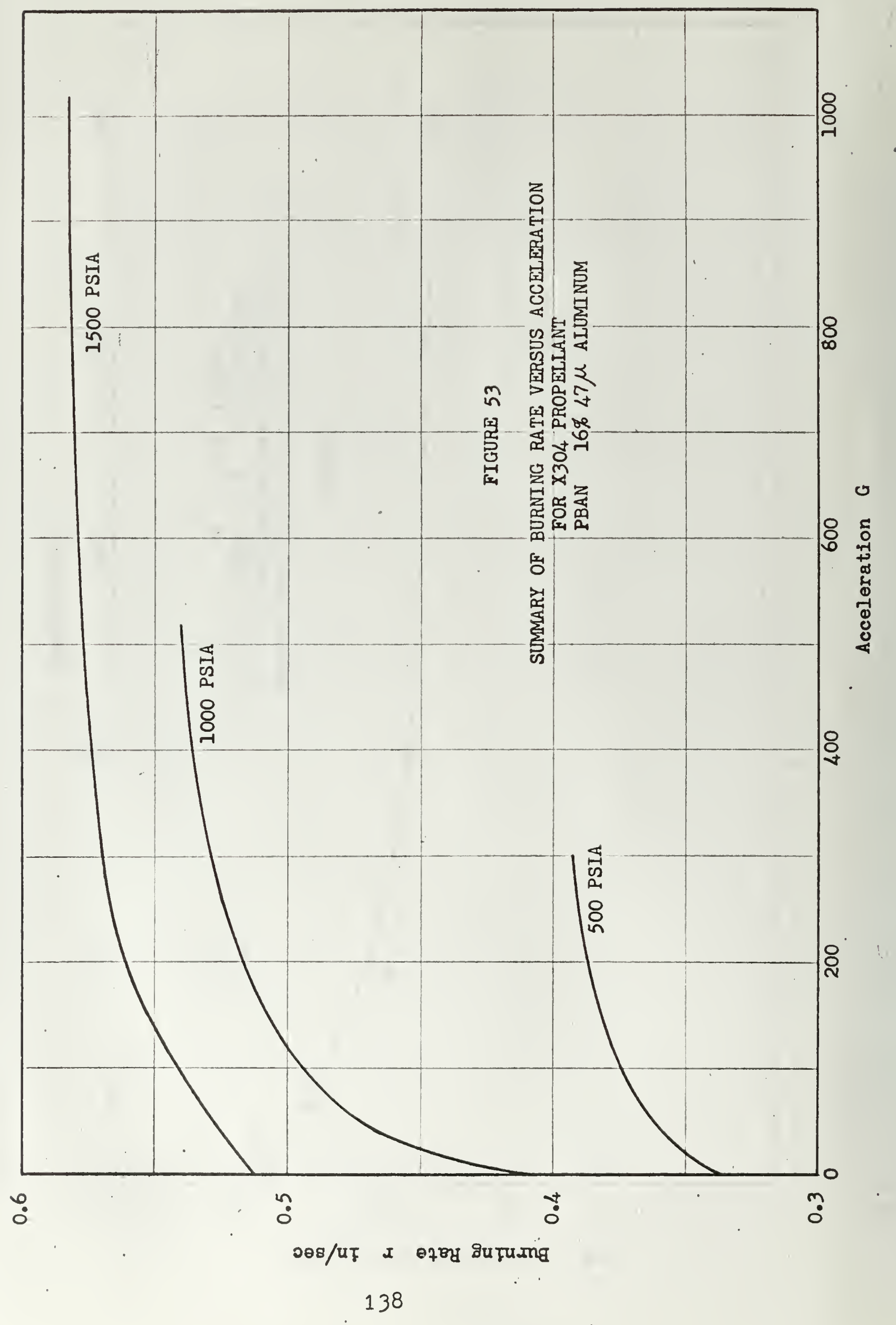




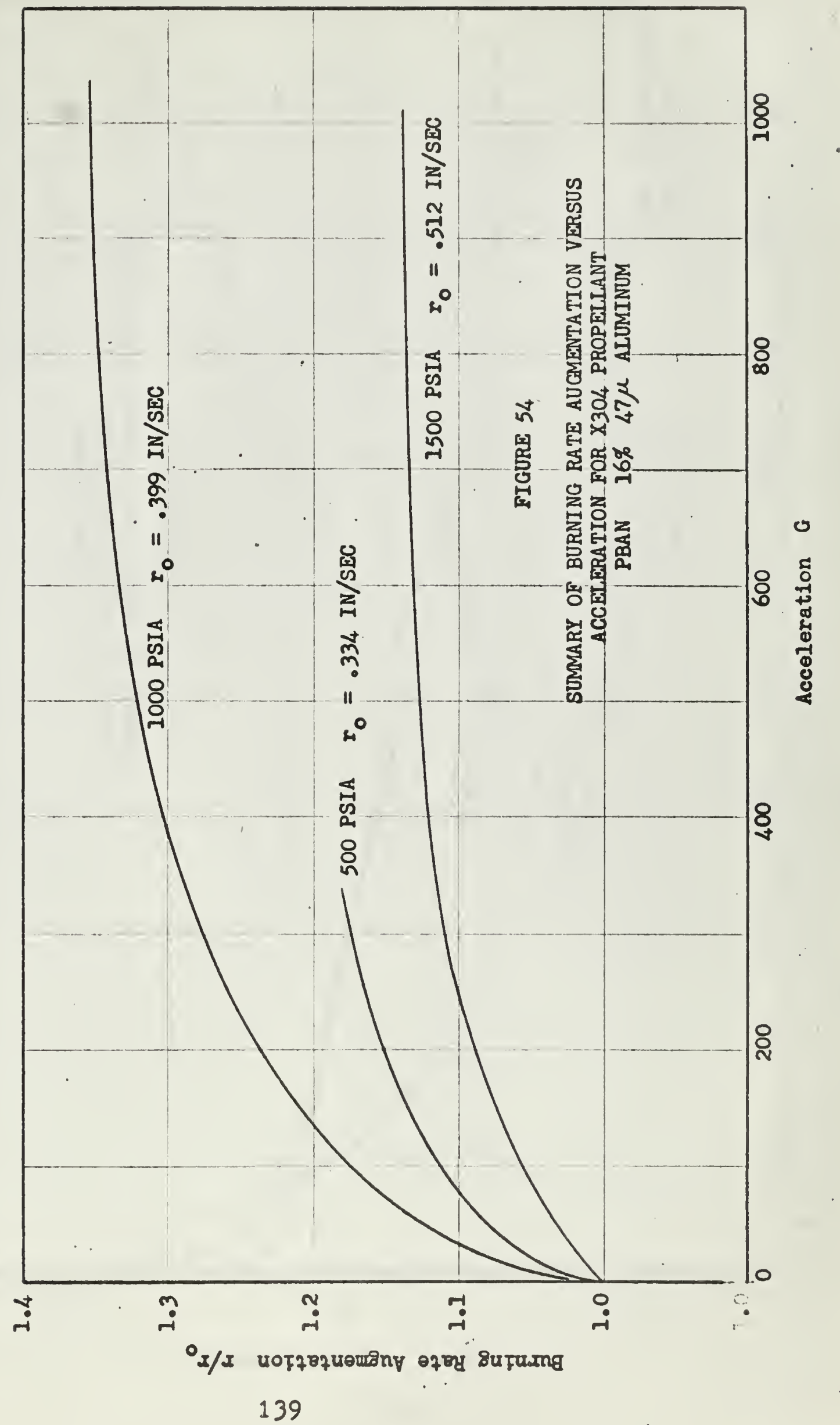




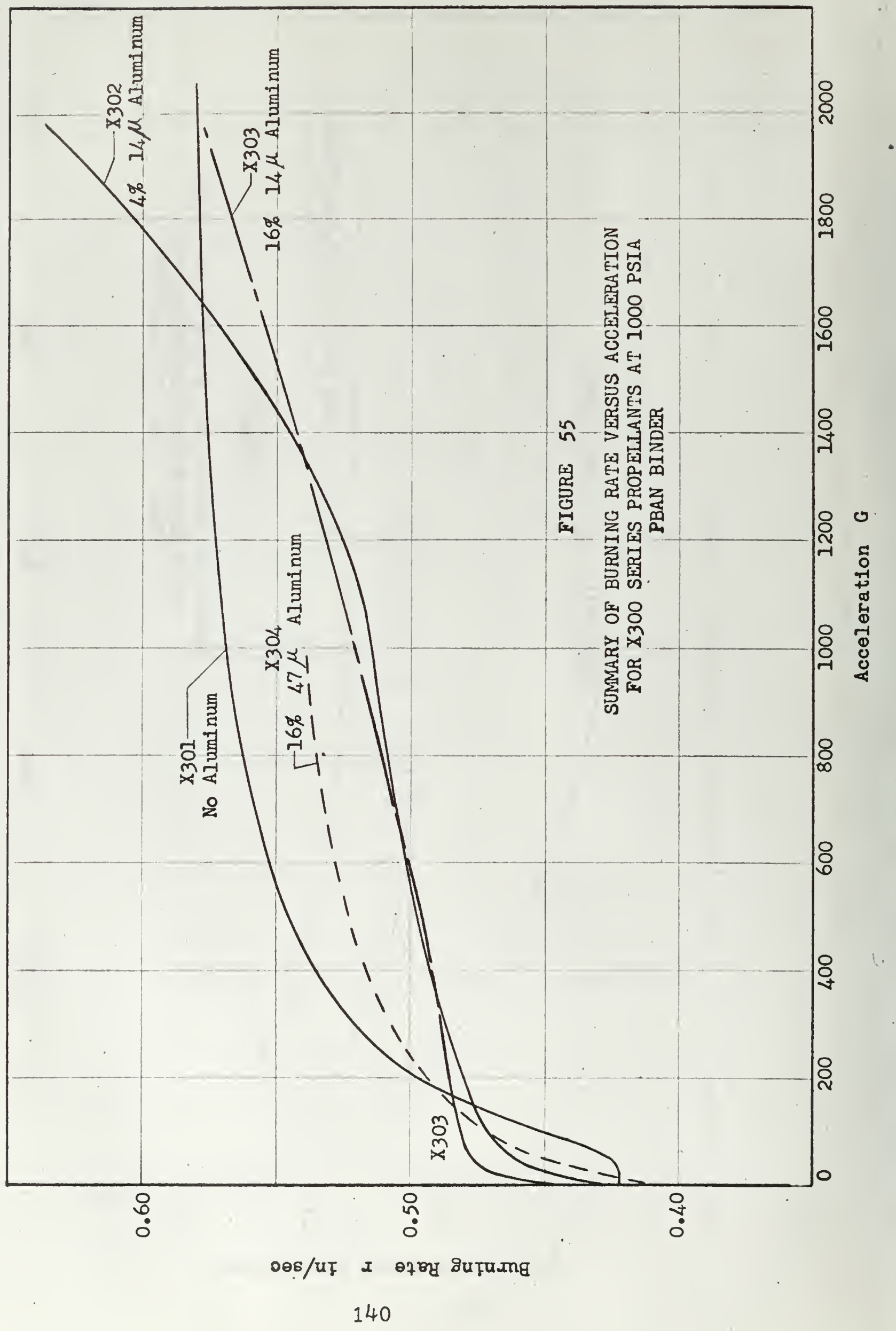




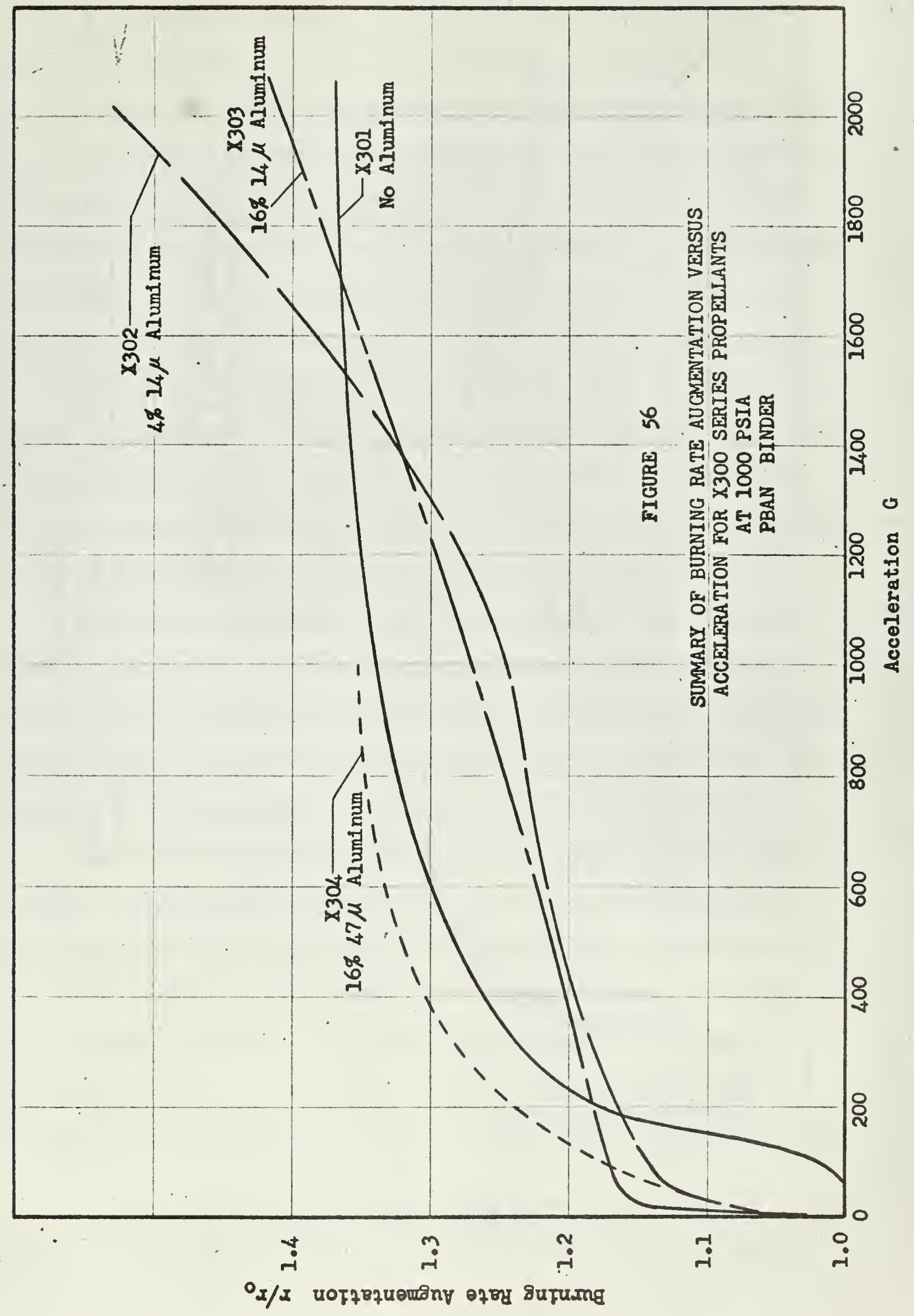




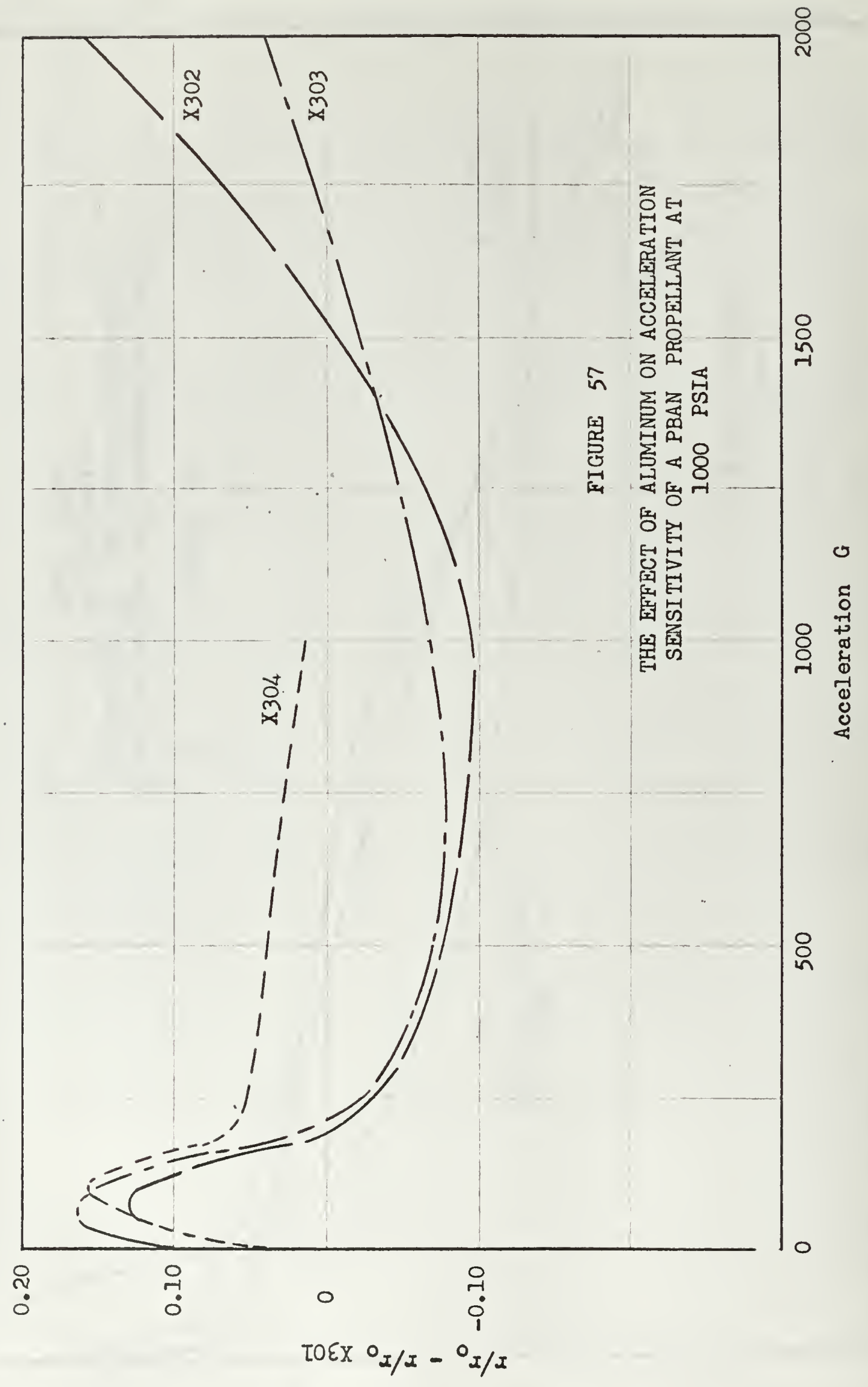


In these instances the ignition wire was placed on the edge of the inhibitor case so that it would not melt in the flame. The lack of an asterisk by a datum point indicates that the ignition wire was placed across the face of the propellant strand and was not intact at the completion of the experiment. The length of the missing segment was about 0.2 inch; and its mass was about $1.34 \mathrm{mg}$. If the wire melted and formed two equal size spheres, ? they would have been about $540 \mathrm{mi-}$ crons in diameter. These spheres, if held against the burning surface by centrifugal force, would have been immersed in the gas-phase reaction zone. Thus they could have served as good conductors of energy to the propellant.

Figures 13 through 20 show considerable burning rate augmentation when the ignition wire was allowed to burn through. At all three pressures the burning rate increased rapidly with acceleration up to about $100 \mathrm{G}$. Above $100 \mathrm{G}$ the burning rate continued to increase, but at a much slower rate. At 1000 psia and above 350G, the propellant strands stopped burning after ignition. In some cases extinguishment was near the beginning, and in some cases near the end. At $1983 \mathrm{G}$ ignition took place, but extinguishment followed after burning about $1 / 16$ inch in the immediate vicinity of the ignition wire. This same strand subsequently burned normally at 1500 psia, zero G. At 1500 psia, the propellants

7 High speed pictures of aluminum wires burning in oxygen indicate that when the wires break, molten spheres form on the wire ends and travel outward toward the wire suspension points. [12] 
burned normally up to 500G. At $1500 \mathrm{G}$ the propellant would not ignite without black powder, and at $1980 \mathrm{G}$ only about $1 / 16$ inch of the strand burned. At 1000 and $1500 \mathrm{G}$ the strands burned completely, but the pressure traces indicated voids in the propellant.

A comparison of the burning rate augmentation at different pressures in Figure 20 shows that for a given acceleration, the augmentation increased with pressure.

When the ignition wire was placed on the edge of the inhibitor case so that it would not melt in the flame, much different results were obtained. At 500 and 1000 psia, there was an increase in burning rate at 100G. At 200G there was a decrease; that is, the burning rate was lower than with zero G. At $300 \mathrm{G}$ the strands stopped burning after about $3 / 8$ inch. No such experiments were run at 1500 psia.

Considerable deposition of a black powdery substance, believed to be condensed phase combustion products, occurred on the inside walls of the inhibitor case at zero G. The amount of deposition decreased rapidly with increasing acceleration, and the material was not accumulated in the capped end of the case after the experiment. It is believed that at the higher burning rates caused by the presence of the nichrome at the burning surface, the gas velocity was sufficient to reduce the deposition of material on the inhibitor case walls.

X102 - 9.7\% 6.3 micron aluminum. The X102 propellant was investigated at 1500 psia only for comparison with other 
variations in the $\mathrm{X} 100$ series. Figures 21 and 22 indicate that the presence of the ignition wire had insignificant effect on the burning rate augmentation. The Figures show a rapid increase in burning rate up to $25 \mathrm{G}$, after which the slope of the curve is much lower. However, the slope increases again above 500G. It is interesting to note that the only other formulations showing increasing acceleration sensitivity above $500 G$ were the $\mathrm{X} 302$ and $\mathrm{X} 303$, both PBAN formulations with 14 micron aluminum.

X103 - $17.7 \% 6.3$ micron aluminum. The X103 propellant was investigated at both 500 and 1500 psia. As shown in Figures 23 through 27 , the initial augmentation is not as rapid as with the $\mathrm{X} 101$ and $\mathrm{X} 102$, and the rather abrupt change in slope is also not present. It should be noted that particularly below 100G the curve indicates only the general trend. It may be that additional data points in the low acceleration range would reveal a rapid augmentation increase up to about 10G, a leveling off, and then another increase in the 50 to $100 \mathrm{G}$ range.

X104-17.7\% 31 micron aluminum. Both 0.2 inch $x 0.2$ inch and 0.4 inch $\times 0.4$ inch $X 104$ strands were used. From the results shown in Figures 28 through 32, it was concluded that within the range of sizes used, the burning rate and burning rate augmentation are not significantly affected by size.

Four 0.2 inch $\times 0.2$ inch strands inhibited with $40 \times 415$ Plastisol Primer (Stanley Chemical Co., East Berlin, 
Connecticut) were burned at $500 \mathrm{psia}$. Two were burned at zero $G$ and two were burned at 20G. The average burning rate at zero $G$ was used to calculate burning rate augmentation at 20G. Figures 28 and 29 show that while burning rate is affected by the inhibitor material, burning rate augmentation apparently is not.

Figure 34 shows that burning rate augmentation increased with pressure $(500,1000$ and 1500 psia) at all accelerations up to $1000 \mathrm{G}$.

Comparison between $\mathrm{X} 100$ series formulations. Figures 35 and 36 show the comparative burning rate change for the $\mathrm{X} 100$ series propellants at 1500 psia. Figure 35 shows actual burning rate and Figure 36 shows burning rate augmentation. of particular interest is the behavior of the non-aluminized propellant, in which the augmentation is attributed to the 1.34 mg. nichrome ignition wire. Except possibly for $G<15$, it showed greater augmentation than any of the aluminized formulations. If it is assumed that the ignition wire, which was by mass $60 \% \mathrm{Ni}, 25 \% \mathrm{Fe}$, and $15 \% \mathrm{Cr}$, reacted with the oxidizer to produce $\mathrm{NiO}, \mathrm{Fe}_{2} \mathrm{O}_{3}$, and $\mathrm{CrO}_{3}$, it can be shown that the energy released would have been only about $1 / 800$ that released by the formation of $\gamma-\mathrm{Al}_{2} \mathrm{O}_{3}$ from all of the aluminum in the X102 propellant. This suggests that an increase in thermal conductivity between the reaction $z$ one and the solid may be an important mechanism. It further indicates that the observed increases in burning rate shown by the aluminized propellants were not due simply to the 
release of additional energy close to the propellant surface.

Also of interest is the comparison of the X102 and the $\mathrm{X} 103$ in Figure 36. The X102, which had half as much aluminum showed more sensitivity to acceleration below $90 \mathrm{G}$ and above 925G. It was anticipated that the $\mathrm{X} 103$ would be more sensitive to acceleration at all values of $G$.

The X104, which had the larger aluminum particle size, was more sensitive than the X103 up to 525G. From that point on, the X103 augmentation was greater. Above 800G, the $\mathrm{X} 104$ showed less augmentation than either the X102 or $\mathrm{X} 103$.

X200 - Carboxy-terminated Polybutadiene Binder, $17.7 \%$

2.1 Micron Aluminum

Figure 37 shows $\log \left(r / r_{0}-1\right)$ as a function of $\log G$ for 500,1000 , and 1500 psia. Unlike the X100 series, there was little pressure dependence associated with acceleration sensitivity. Also, the augmentation at 1000 G was only 1.23 , while the $\mathrm{X} 103$ showed an augmentation of 3.8 . This indicates strong dependence of augmentation on oxidizer particle size distribution and/or binder material.

\section{$\underline{x 300 \text { Series - PBAN Binder }}$}

X301 - no aluminum. Figures 38,39 , and 40 show burning rate augmentation as a function of $G$ at pressures of 500,1000 , and 1500 psia respectively. The asterisk (*) denotes that the ignition wire was still intact after the 
strand holder was removed from the bomb. The Figures show this to be a significant factor below 200G. When the wire remained intact, the propellant burning rate was insensitive to acceleration up to about 100G. At 1000 psia this threshold appears to lie between 75 and 100G. As acceleration was increased, the augmentation increased rapidly and then leveled off. This is in sharp contrast to the X101 where acceleration resulted in propellant extinguishment at $300 \mathrm{G}$ and greater.

At about $300 \mathrm{G}$ and greater, the disposition of the ignition wire appears to be insignificant. i.e., there was little difference, if any, between the points with and without (*). This indicates that the mechanism responsible for the augmentation increase remained rate controlling in spite of the presence of the ignition wire. This also is in contrast with the X101.

It is of interest to note that the pairs of data points on Figure 38 marked 1 and 2, 3, and 4, and 5 and 6 are in the same acceleration ranges, yet show quite different burning rate augmentation. Ambient temperature and bomb temperature for these experiments were:

\begin{tabular}{|c|c|c|}
\hline Datum Point & Ambient ${ }^{\circ} \mathrm{C}$. & Bomb ${ }^{\circ} \mathrm{C}$ \\
\hline 1 & 17 & 19 \\
\hline 2 & 19 & 20 \\
\hline 3 & 23 & 25 \\
\hline 4 & 24 & 25 \\
\hline 5 & 22 & 23 \\
\hline 6 & 23 & 24 \\
\hline
\end{tabular}


Experiments 3 and 4 , and 5 and 6 were done in sequence on two different days. Experiments 1 and 2 were done on the same day, but not in sequence. Both the strand holder and propellant sample were in all six instances at a uniform temperature of $20^{\circ} \mathrm{C}$. When removed from the refrigerated oven. Elapsed time from removal from the oven to installation in the bomb was about 30 seconds, and elapsed time from installation in the bomb to ignition was two to three minutes. Hence the propellant temperature at the time of ignition should have been close to $20^{\circ} \mathrm{C}$. The spread in the data points marked 1 through 6 indicates the possibility of significant temperature dependence of acceleration sensitivity in the threshold range.

Figure 42 shows the comparative augmentation for 500 , 1000 , and $1500 \mathrm{psia}$. The curves are essentially the same up to 300G. At higher accelerations the augmentation decreased with increasing pressure. This trend is opposite to that shown by the X101 propellant.

X302 - 4\% 14 micron aluminum. Results for the X302 propellant at 1000 psia are shown in Figures 43 and 44 . The shape of the curve is similar to the X102, also a lightly loaded propellant. The high burning rates at $1500 \mathrm{G}$ and greater suggest voids in the propellants, but in all three runs the pressure traces indicated even burning. X303-16\% 14 micron aluminum. Results for the X303 are shown in Figures 45 and 46 . The data show a rapid increase in burning rate up to about 50G where there is an 
abrupt decrease in slope. At higher accelerations approaching $2000 \mathrm{G}$ a gradual increase in slope is indicated. However, the increase is not nearly so great as with the $\mathrm{X} 302$. X304-16\% 47 micron aluminum. Results for the X304 propellant are shown in Figures 47 through 54. Both $3 / 8$ inch and $\frac{1}{4}$ inch diameter strands were used. As with the $\mathrm{X} 104$, the results indicate no apparent difference in burning rates for the two sizes. The burning rate augmentation at 500 , 1000 , and 1500 psia is shown in Figure 54. A comparison of the curves shows the augmentation to be greatest at 1000 psia, the least at 1500 psia with the 500 psia curve lying approximately midway between. This behavior is different than either of the two propellants (X100 series and X200) previously discussed.

\section{Comparison between $X 300$ series formulations. Figures}

55 and 56 show curves for all four formulations at 1000 psia. In Figure 56 it appears that up to 50G the augmentation of the X302 and the X304 were the same. Above 50G the X304 was greater, but it appears that this situation would have reversed at about 1500 .

The $\mathrm{X} 303$ showed the highest augmentation up to $100 \mathrm{G}$ where the X303 and X304 curves cross. From 200 to $1400 G$ the X303 and X302 were about the same with the X303 higher. Above $1400 \mathrm{G}$ the $\mathrm{X} 302$, with $4 \%$ aluminum, showed greater augmentation than the X303, with $16 \%$ aluminum.

A comparison of the $\mathrm{X} 301$ and $\times 304$ shows that the increase in augmentation was due entirely to the presence of 
aluminum up to about 100G. As acceleration was increased, the contribution from the aluminum became less and less important.

Of special interest is the comparison between the $X 301$ and the propellants with small aluminum particles (X302 and $\times 303)$. As with the $\times 304$, the increase in augmentation appears to have been caused by the aluminum up to $100 \mathrm{G}$. Above $200 \mathrm{G}$ however, the augmentation was greater for the non-aluminized X301. Between 1500 and 1700G the curves cross, so that again the aluminized propellants show the greater acceleration sensitivity.

The curves in Figure 57 are presented in order to more clearly show the role of aluminum in acceleration sensitivity. The curves are derived from Figure 56 by subtracting $r / r_{0}$ for the non-aluminized X301 from the aluminized propellants.

\section{Time Dependence}

Spinning motor experiments have indicated an apparent time dependence of acceleration effects. [6] This may be seen in Figure 4b. As a result of these findings, similar behavior was anticipated in the present investigation. However, the burning rate summaries shown in Tables II and III do not show any consistent time dependence. This disagreement may be due to the different burning lengths in the two investigations. The spinning motor [6] had a web thickness of 0.6 inch while the strands in Tables II and III were $2 \frac{1}{4}$ 
TABLE II

BURNING RATE SUMMARY

X104 PROPELLANT 500 PSIA

\begin{tabular}{|c|c|c|c|c|}
\hline $\begin{array}{c}\text { Acceleration } \\
G\end{array}$ & $\begin{array}{c}r_{1} \\
\text { in/sec }\end{array}$ & $\begin{array}{c}r_{2} \\
\text { in/sec }\end{array}$ & $\begin{array}{c}r_{3} \\
\text { in/sec }\end{array}$ & $\begin{array}{c}\bar{r} \\
\text { in/sec }\end{array}$ \\
\hline 0 & 0.145 & 0.145 & 0.143 & 0.144 \\
9 & .132 & .141 & .140 & .137 \\
16 & .202 & .177 & .155 & .176 \\
32 & .208 & .174 & .161 & .179 \\
49 & .188 & .188 & .179 & .185 \\
103 & .186 & .184 & .180 & .183 \\
106 & .209 & .229 & .224 & .221 \\
214 & .203 & .201 & .202 & .202 \\
316 & .228 & .230 & .234 & .231 \\
622 & .284 & .272 & .261 & .275 \\
1034 & .316 & .318 & .326 & .320 \\
\hline
\end{tabular}

$r_{1}$ is the average burning rate over the first of three adjacent intervals, each $\frac{1}{2}$ inch long.

$$
\bar{r}=\left(r_{1}+r_{2}+r_{3}\right) / 3
$$


TABLE III

BURNING RATE SUMMARY

X304 PROPELLANT 500 PSIA

\begin{tabular}{|c|c|c|c|c|}
\hline $\begin{array}{c}\text { Acceleration } \\
G\end{array}$ & $\begin{array}{c}r_{1} \\
\text { in/sec }\end{array}$ & $\begin{array}{c}r_{2} \\
\text { in/sec }\end{array}$ & $\begin{array}{c}r_{3} \\
\text { in/sec }\end{array}$ & $\begin{array}{c}\bar{r} \\
\text { in/sec }\end{array}$ \\
\hline 0 & 0.334 & 0.345 & 0.343 & 0.340 \\
0 & .338 & .345 & .342 & .342 \\
0 & .317 & .317 & .316 & .317 \\
0 & .324 & .330 & .334 & .329 \\
21 & .331 & .363 & .359 & .352 \\
39 & .382 & .370 & .338 & .363 \\
96 & .372 & .351 & .346 & .355 \\
101 & .378 & .407 & .352 & .378 \\
101 & .380 & .376 & .367 & .378 \\
103 & .386 & .374 & .370 & .382 \\
204 & .379 & .376 & .370 & .375 \\
207 & .434 & .384 & .393 & .402 \\
306 & 0.414 & 0.373 & 0.366 & 0.384 \\
\hline
\end{tabular}

$r_{1}$ is the burning rate over the first of three adjacent intervals, each $3 / 4$ inch long.

$$
\bar{r}=\left(r_{1}+r_{2}+r_{3}\right) / 3
$$


and $23 / 4$ inches long. Hence an initial transient condition would be more apparent in the spinning motor investigation. The Effect of Acceleration on Propellant Burning Rate

\section{Exponent}

An empirical relationship commonly used to relate propellant burning rate sensitivity to combustion chamber pressure is $r=a P^{n}$ where $(r)$ is burning rate and $(P)$ is pressure. The coefficient (a) and the pressure exponent ( $n$ ) are constant over a limited range of pressure, the extent of the range being dependent on the propellant. In Figures 58 and 59 it has been assumed that this relationship is valid in the acceleration field environment.

Figure 58 shows the variation of the pressure exponent (n) with G for the X101, X103, and X104 propellants. The burning rate increase in the $\mathrm{X} 101$ is attributed to the 1.34 $\mathrm{mg}$ of nichrome wire. The general tendency was for $(n)$ to be greater with acceleration than without. With X101 and X103, the value of $(n)$ continued to increase as acceleration was increased. The X104, however, showed a decreasing value of $(n)$ as acceleration was increased beyond $50 \mathrm{G}$.

Figure 59 shows the variation of $(n)$ with $G$ for the X301 and X304 propellants. Both the X301 and X304 showed little change in burning rate pressure dependence with accel-. eration.

The curves in both Figures 58 and 59 were derived from the smooth curves drawn through the burning rate data. 

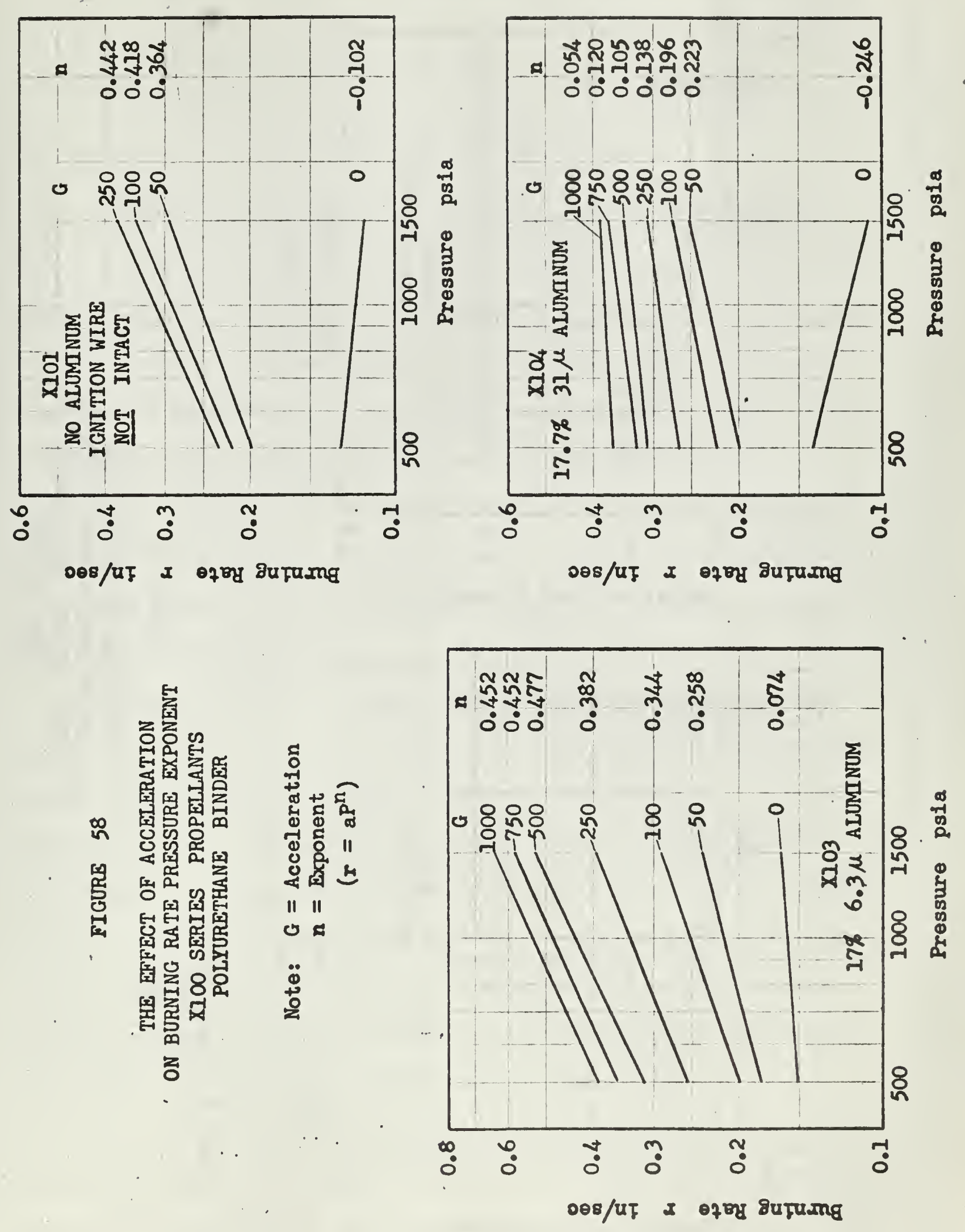

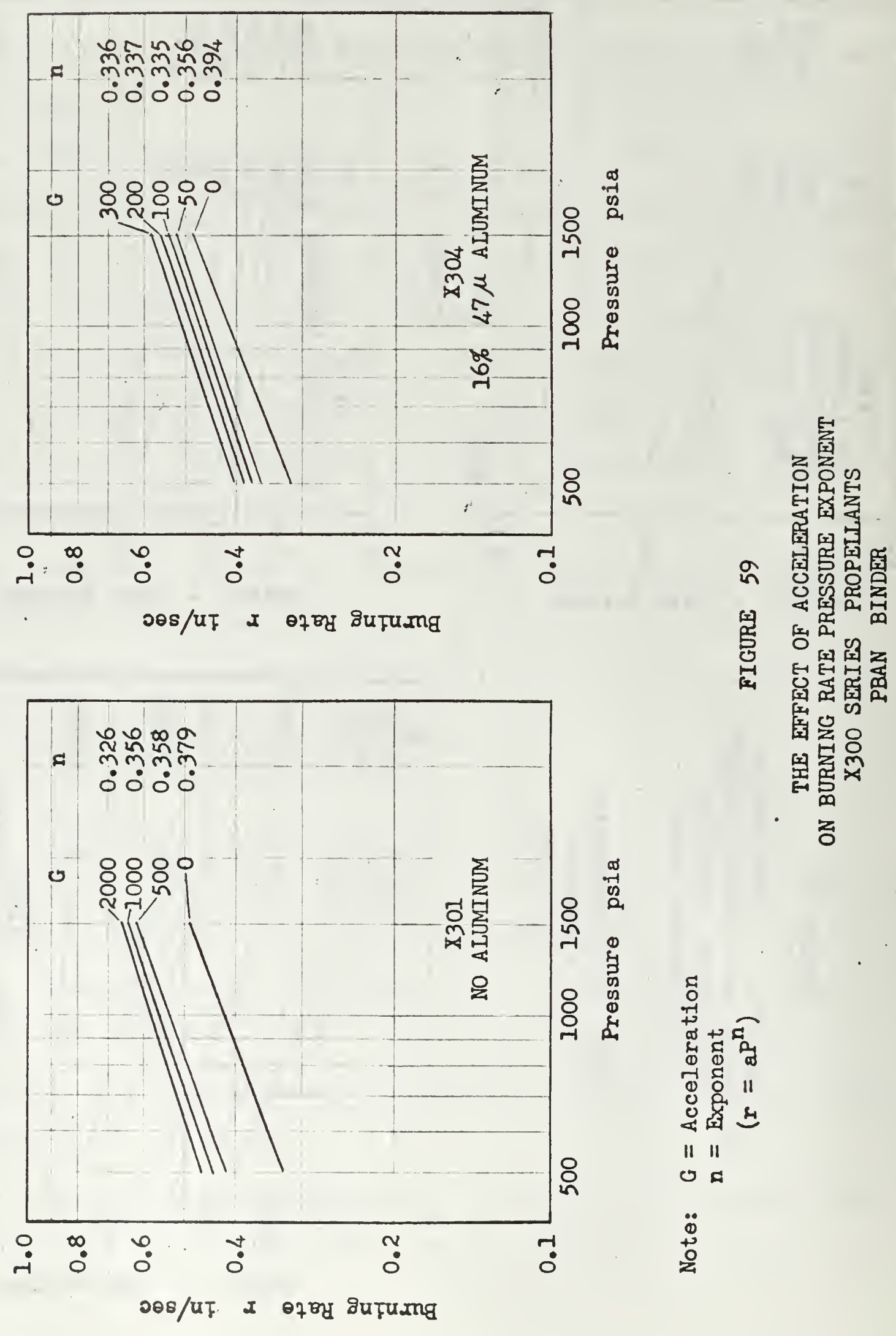
The X200 propellant burning rate pressure exponent was insensitive to acceleration provided that the curve shown in Figure 37 is taken to be a reasonable fit to the data.

\section{RESIDUE ANALYSIS}

Inhibitor cases from the metallized propellant experiments were periodically broken open during the course of the investigation to inspect for possible residue. The residues of all three propellant series, X100, X200, and X300 were similar in appearance. Deposited on the inside walls of the inhibitor cases was a powdery carbon-like substance. A metallic material was found in the bottom of the inhibitor cases, and the amount of this metallic material appeared to be proportional to the acceleration level at which the propellant had been burned.

Sixteen inhibitor cases were selected for closer inspection. Ten $\mathrm{X} 104$ samples and six X200 samples, representing accelerations of zero to $1000 \mathrm{G}$, were inspected. Inside the cases there was found a powdery carbon-like substance clinging to the inside walls and deposited at the closed end of the case. The amount of material deposited at the closed end increased as acceleration increased but its appearance did not change. No measurements of particle size or chemical composition of this material were made.

Also found inside the cases was what appeared to be a metal or metal oxide. Subsequent x-ray diffraction and infrared spectrophotometer analyses indicated this material to be 
predominantly aluminum oxide. In the case of zero $G$, these oxide particles were imbedded in the black powder on the case walls. The particles were solid, spherical in shape, and the largest were approximately 1000 microns in diameter. At 8G (X200 propellant) there were a few spherical particles found on the walls but they were considerably smaller than 1000 microns. In the bottom of the case, however, there was found a single spheroidal particle about $1 / 8$ inch in diameter. This particle had a mass of 38.0 milligrams and is shown in Figure 60. The size of the X200 propellant sample in this particular experiment was 0.39 inch $\mathrm{x} 0.40$ inch $\mathrm{x}$ 2.283 inches. As acceleration increased, oxide particles ceased to appear on the case walls but the amount of residue found in the bottom of the case increased. From 10G (X104 propellant) to about 300G (X104 and X200 propellants) the oxide in the bottom of the case was in several pieces. The pieces appeared to have come from one or more larger masses which apparently suffered multiple fractures during cooling. The epoxy cap on which the particles were resting generally had one area of severe charring which increased in size as the acceleration increased.

Above $300 \mathrm{G}$ the oxide residue in the bottom could be removed in one piece, although multiple cracks through the material could be observed. The thickness of the layer increased with acceleration. At $1014 \mathrm{G}$ (X104 propellant) the solid piece of oxide was about 0.16 inch thick and is shown in Figures 60 and 61. The mass of the oxide shown in 

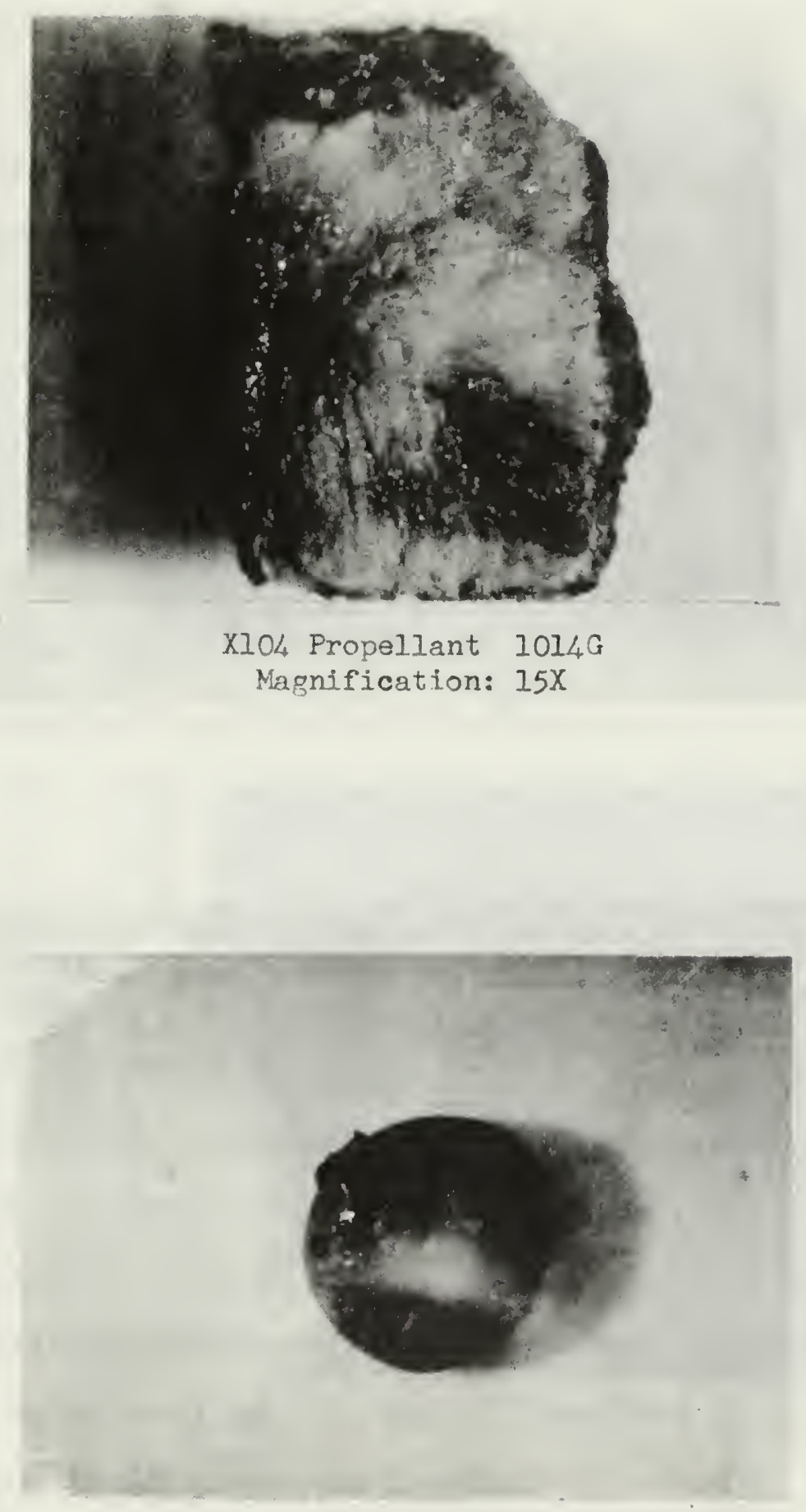

X200 Propellant $8.3 G$

Magnification: $10 \mathrm{X}$

\author{
FIGURE 60
}

PROPELLANT RESIDUES 

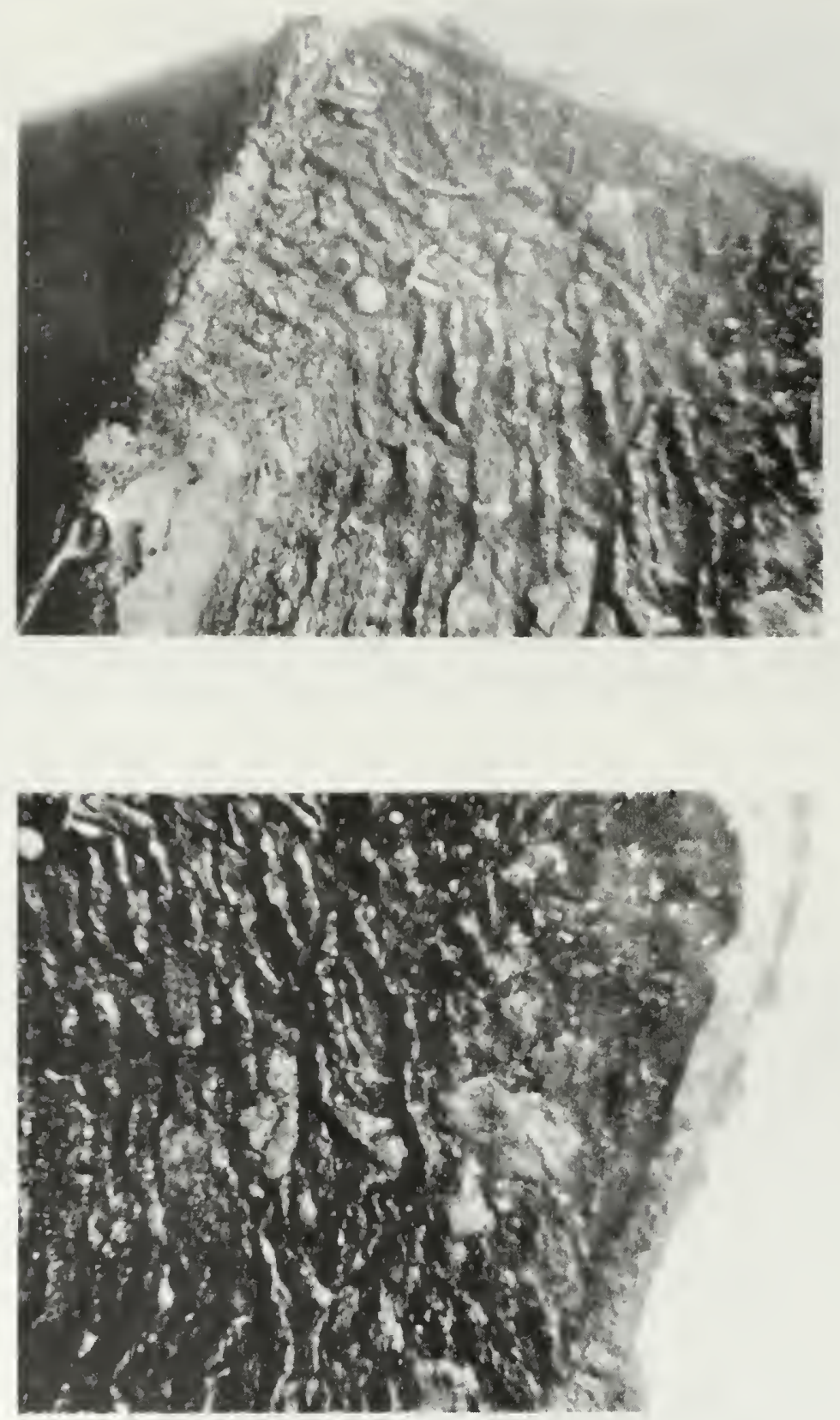
Figure 61 is 1.816 grams. The $\mathrm{X} 104$ propellant sample was 0.4 inch $\times 0.4$ inch $\times 2.245$ inches.

In some instances there was attached to the periphery of this block of material a wall up to $3 / 8$ inch high. It appeared to be of the same material and was about $1 / 64$ inch thick. This formation occurred more in the $\mathrm{X} 104$ than the X200. A wall with a mass of 471 milligrams was attached to the oxide shown in Figure 61. The formation of this wall is believed to be the result of molten residue material being thrown out of the pool by the combustion gases and then freezing on the walls of the inhibitor case as it runs back toward the propellant surface.

The oxide residue collected from the bottom of each inhibitor case was weighed on an automatic laboratory balance (Mettler Model H15) to determine its mass. The results are shown in Figure 62: In calculating the ordinate it was assumed that the residue consisted entirely of aluminum oxide $\left(\mathrm{Al}_{2} \mathrm{O}_{3}\right)$. The aluminum retention ratio is equal to $0.529 \mathrm{x}$ residue mass/total mass of aluminum in the propellant sample. The major portion of the scatter in the lower acceleration range is attributed to the difficulty encountered in separating the small fractured oxide particles from the black powdery residue.

\section{X-Ray Diffraction Analysis}

Two samples of $\mathrm{X} 200$ residue and two samples of $\mathrm{X} 104$ residue were analyzed with a Norelco x-ray diffractometer using nickel-filtered copper radiation. Scan rate $(2 \theta)$ 


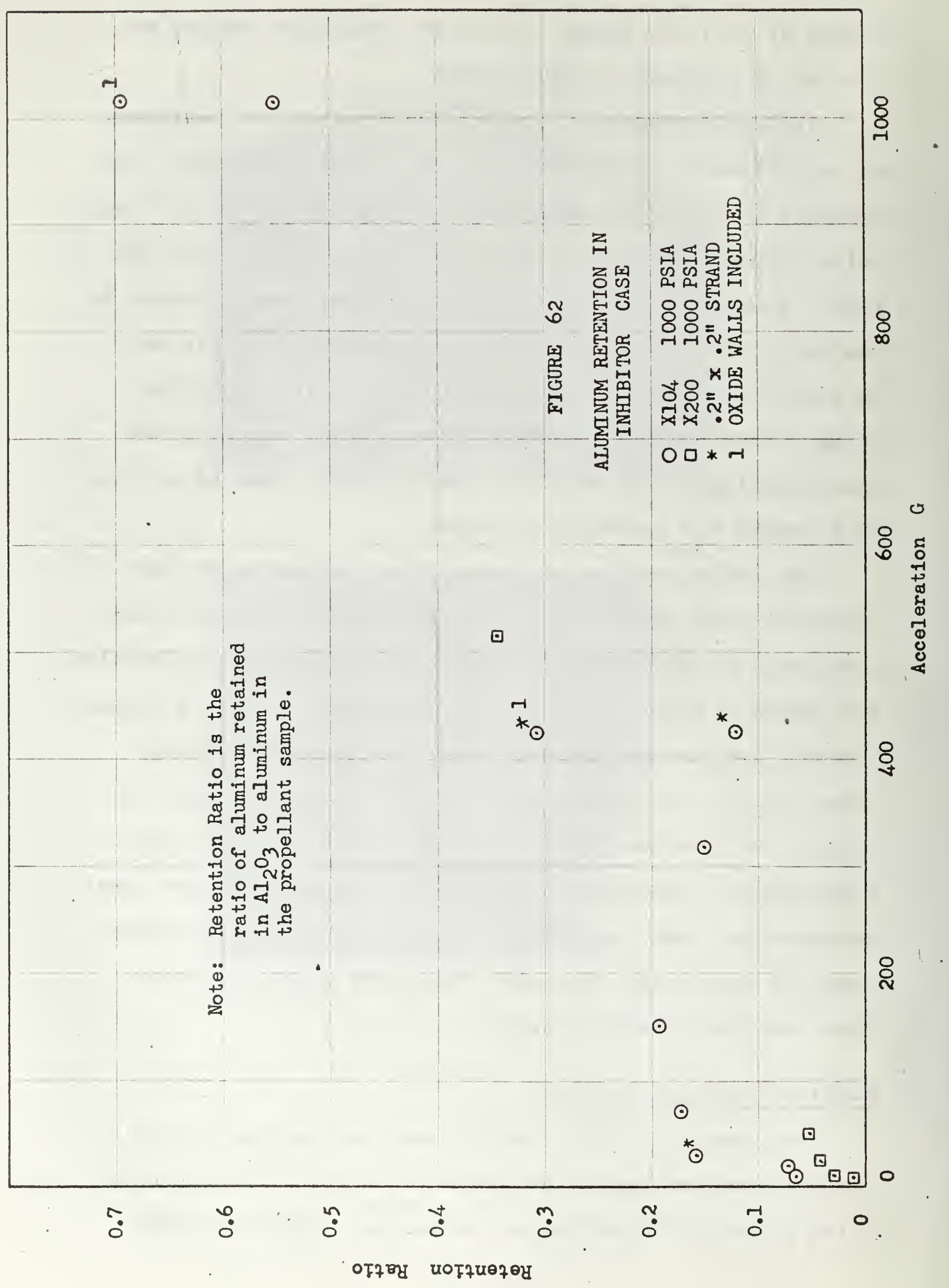


was one degree per minute from 70 degrees to five degrees. Chart speed was $\frac{1}{2}$ inch per minute.

The X200 propellant residues were from burning rate experiments at 8.3 and 515G. The $\mathrm{X} 104$ propellant residues were from burning rate experiments at 72 and 1014G. Diffractometer charts were also run for Baker reagent grade $\mathrm{Al}_{2} \mathrm{O}_{3}$ powder. The diffractometer charts are shown in Figures 63,64 , and 65 .

The interplaner spacing values ( $d$ values) corresponding to the strongest intensities in the diffraction patterns were compared with those tabulated in the Index to the X-Ray Powder Data File (1960) [13] to determine the probable composition. It was deduced that in every case the material was predominantly alpha and gamma aluminum oxide. Some of the diffraction patterns have peaks of lesser intensity possibly due to the presence of delta and theta aluminum oxide. Peaks in the diffraction pattern corresponding to pure aluminum were sought but not found.

The diffraction pattern for the reagent grade $\mathrm{Al}_{2} \mathrm{O}_{3}$ shown in Figure 64 is quite similar to all of the residue patterns.

\section{Infrared Spectrophotometer Analysis}

A sample of $\mathrm{X} 200$ propellant residue from the burning rate experiment at $515 \mathrm{G}$ was also analyzed using a Perkin Elmer Model 621 Grating Infrared Spectrophotometer. Figure 66 shows the absorbtion spectra for three test wafers. Two were made using pulverized residue from the X200, 515G 


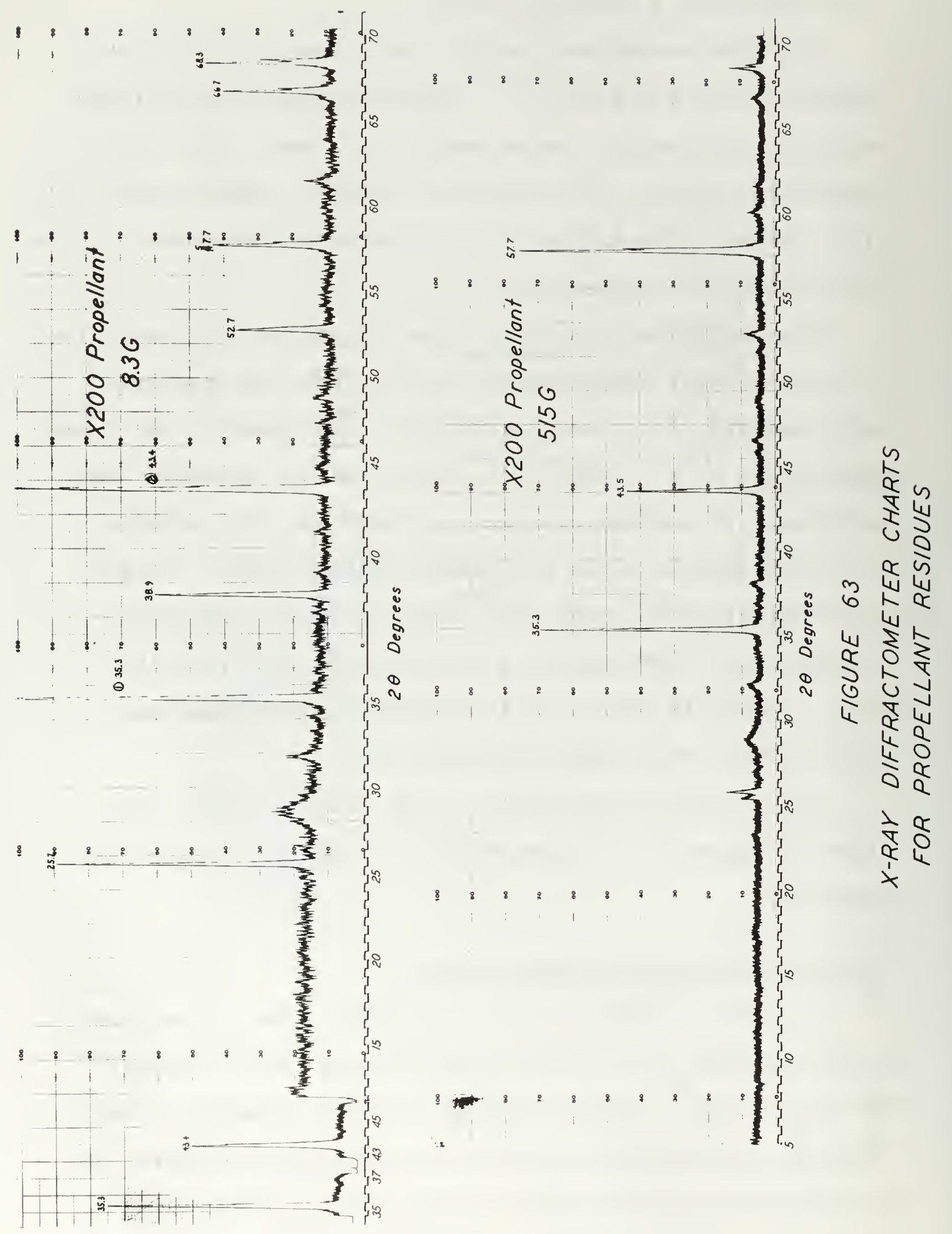




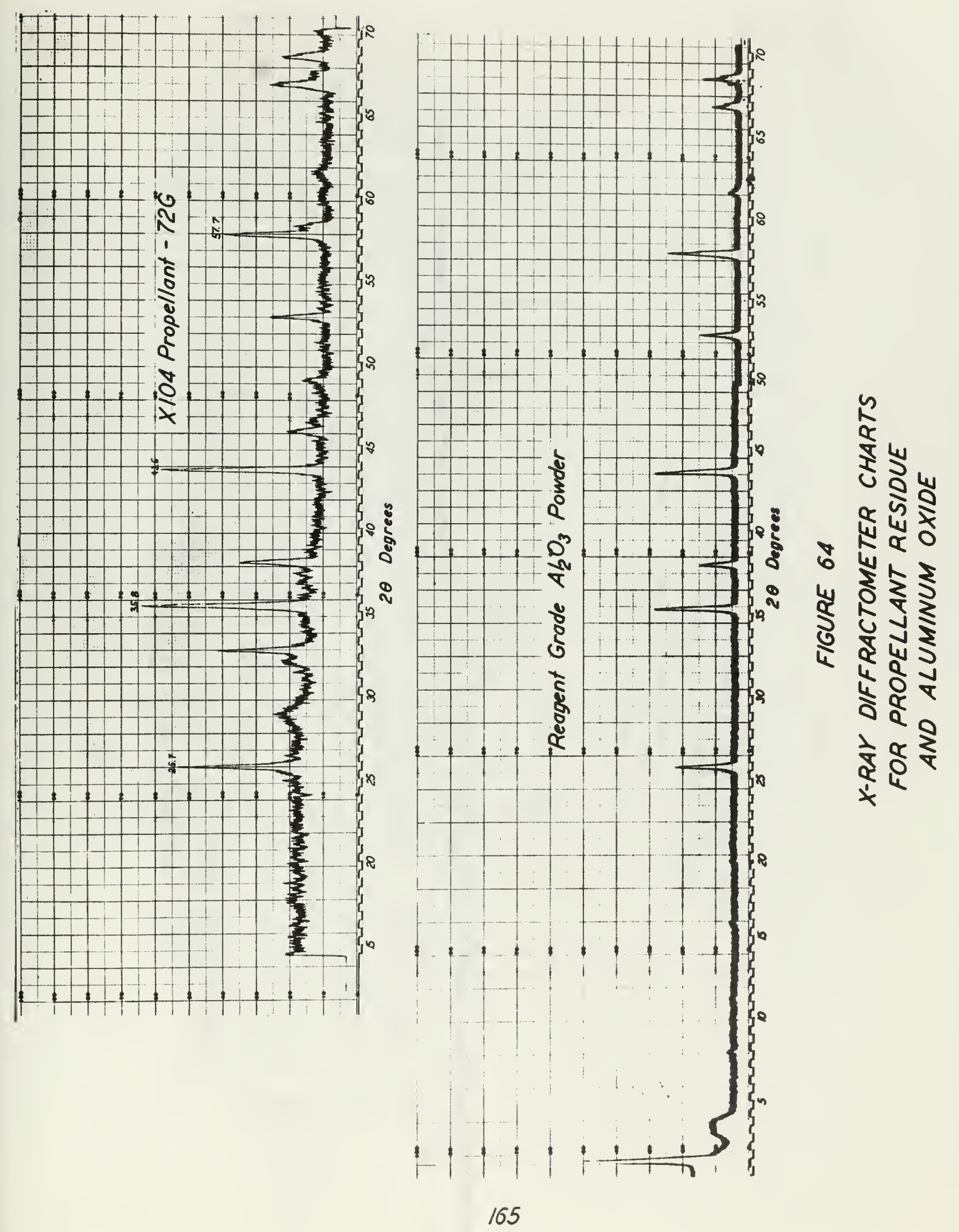




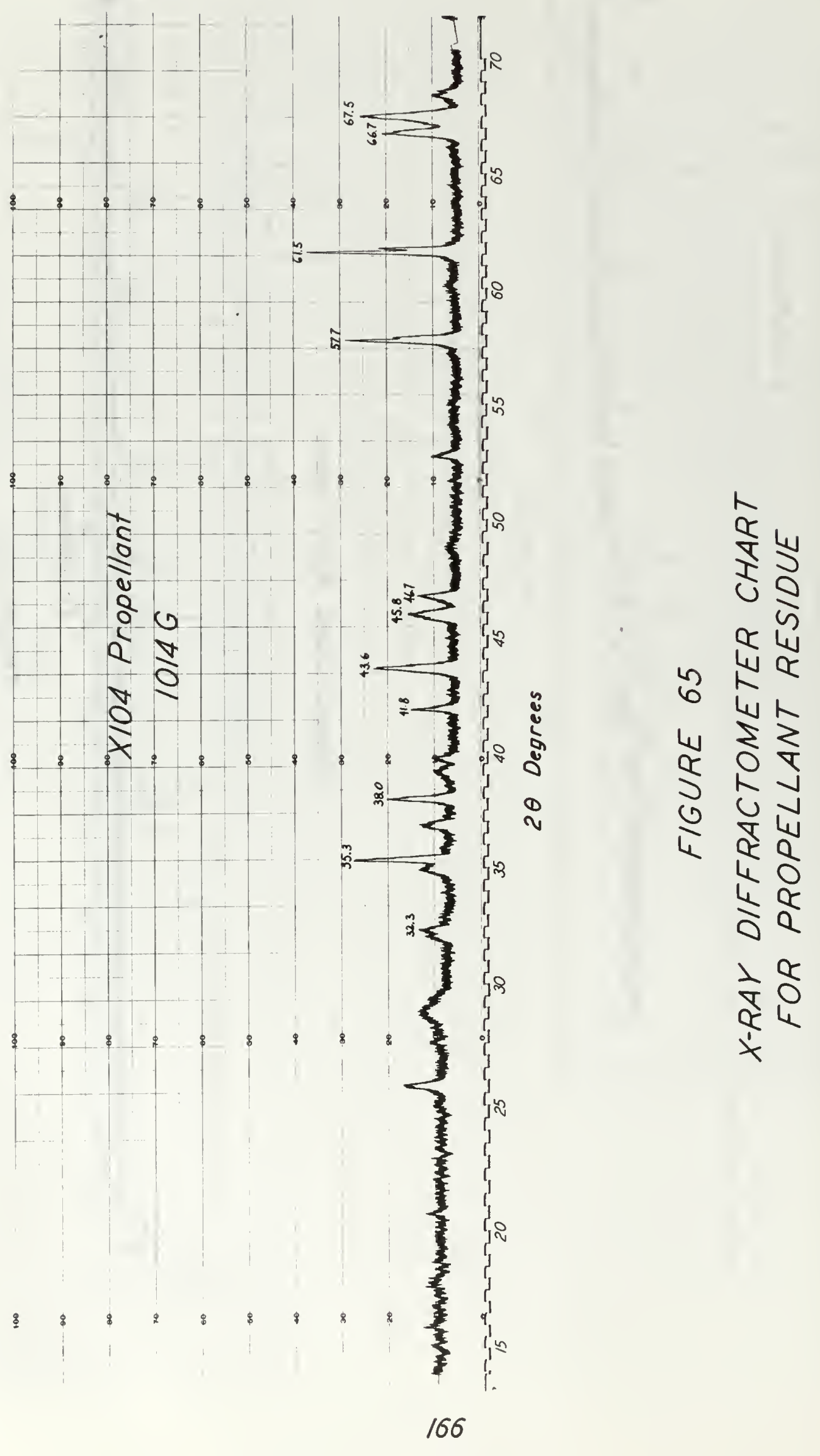




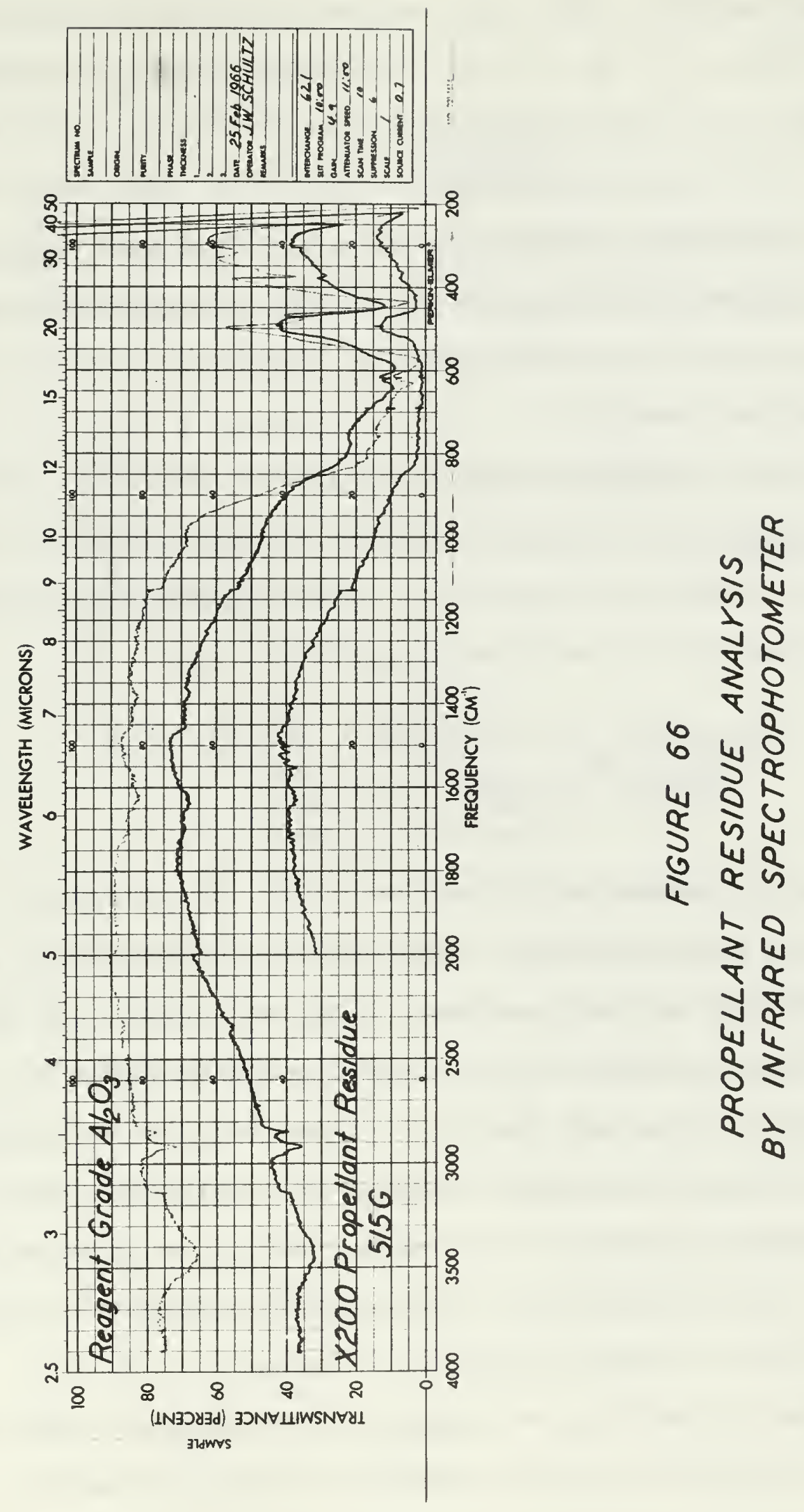


propellant residue. The first was made with a specimen to $\mathrm{KBr}$ mass ratio of 1 to 100 and the second with a mass ratio of 1 to 300. As can be seen in Figure 66 the 1 to 300 wafer resulted in the more meaningful trace. The third test wafer was made from Baker reagent grade $\mathrm{Al}_{2} \mathrm{O}_{3}$. The mass ratio of $\mathrm{Al}_{2} \mathrm{O}_{3}$ to $\mathrm{KBr}$ was 1 to 300 . The similarity between the upper two traces shows the predominance of aluminum oxide in the X200 propellant residue.

From the results of this analysis and the $x$-ray diffraction analyses it is concluded that the completeness of the chemical reaction between the aluminum and the oxidizer is unaffected by acceleration up to $2000 \mathrm{G}$.

\section{COMPARISON OF EXPERIMENTAL RESULTS WITH} PROPOSED THEORETICAL MODELS

Crowe's critical particle size model [6], predicts a burning rate increase due to the release of additional energy at the propellant surface. The results of this investigation are similar to the curves based on Crowe's model which are shown in Figure 3. That is, the data indicate a fairly rapid initial increase in burning rate as acceleration is increased from zero. At high acceleration the data in the majority of cases appear to approach a limiting value as dictated by Crowe's theory.

The theory predicts no change in burning rate until the critical acceleration of the largest aluminum particles 
is reached. Assuming Stokes' flow, the critical particle diameter $d_{\text {crit }}$ is given by

$$
d_{\text {crit }}=\left(\frac{18 \mu_{\mathrm{g}} \mathrm{g}}{\mathcal{\rho}_{\mathrm{p}} \mathrm{g}}\right)^{\frac{1}{2}}
$$

Reasonable values of the variables in Equation (22) for a typical aluminized propellant burning at 1000 psia are

$$
\begin{aligned}
r & =1 \mathrm{~cm} / \mathrm{sec} \\
\rho_{s} & =1.6 \mathrm{gm} / \mathrm{cm}^{3} \\
z & =0.2=\text { aluminum mass fraction } \\
\rho_{\mathrm{p}} & =\text { aluminum particle density } \simeq 2.7 \mathrm{gm} / \mathrm{cm}^{3} \\
\mathrm{~T} & =3000^{\circ} \mathrm{K} \\
\mu_{\mathrm{g}} & =6.6 \times 10^{-4} \mathrm{gm} / \mathrm{cm} \mathrm{sec} \\
\rho_{\mathrm{g}} & =6.1 \times 10^{-3} \mathrm{gm} / \mathrm{cm}^{3} \\
U_{\mathrm{g}} & =\mathrm{r} \rho_{\mathrm{s}}(1-\mathrm{z}) / \rho_{\mathrm{g}}=2.15 \times 10^{2} \mathrm{~cm} / \mathrm{sec} \\
\mathrm{a} & =\text { acceleration }\left(\mathrm{cm} / \mathrm{sec}^{2}\right)
\end{aligned}
$$

The resultant critical particle diameter for a typical aluminized propellant in a one G gravitational field is about 200 microns. Crump's motion pictures [9] taken of propellants burning in a one G field show aluminum agglomerates with diameters in the 100 to 300 micron range, hence an acceleration of $10 \mathrm{G}$ would be expected to have a significant effect on the burning rate. The experimental results of this investigation show that this is the case.

Comparing Crowe's theory with Figure 36, we see that the theory predicts the observed trend with a change in aluminum mass loading between 100 and 900G. Beyond these Iimits however, the observed trends are not predicted. The $\mathrm{X} 302$ 
and $\times 304$ propellants contain $4 \%$ and $16 \%$ aluminum respectively. Hence, according to Crowe's theory the X30 4 propellant should exhibit greater augmentation than the X302. However, Figure 56 shows the same burning rate augmentation for the X302 and the X304 propellants up to 50G. Moreover, the multiple crossing of curves in Figure 56 is not in accord with the theory.

Crowe's theory also predicts a change in burning rate augmentation with pressure to the extent that particle drag is affected. Assuming Stokes' flow, the drag force $F_{d}$ on a spherical particle at rest on the propellant surface is given by

$$
F_{d}=3 \pi \mu_{g} d U_{g}
$$

where

$$
\begin{aligned}
\mu_{\mathrm{g}} & =\text { gas viscosity }(\mathrm{gm} / \mathrm{cm} \mathrm{sec}) \\
\mathrm{d} & =\text { particle diameter }(\mathrm{cm}) \\
\mathrm{U}_{\mathrm{g}} & =\text { gas velocity }=\mathrm{r}(1-z) \rho_{\mathrm{S}} / \rho_{\mathrm{g}}(\mathrm{cm} / \mathrm{sec}) \\
\mathrm{r} & =\text { propellant burning rate }(\mathrm{cm} / \mathrm{sec}) \\
\rho_{\mathrm{S}} & =\text { density of solid propellant }\left(\mathrm{gm} / \mathrm{cm}^{3}\right) \\
\rho_{\mathrm{g}} & =\text { gas density }\left(\mathrm{gm} / \mathrm{cm}^{3}\right) \\
z & =\text { aluminum mass fraction }
\end{aligned}
$$

The body force $F_{b}$, which opposes the drag force, is

$$
F_{b}=\frac{\pi a^{3}}{6} \rho_{p} a
$$

where

$$
\begin{aligned}
\rho_{\mathrm{p}} & =\text { particle density }\left(\mathrm{gm} / \mathrm{cm}^{3}\right) \\
a & =\text { acceleration }\left(\mathrm{cm} / \mathrm{sec}^{2}\right)
\end{aligned}
$$


If the acceleration is greater than the critical acceleration for a given particle, then $F_{b}>F_{d}$, and the particle will remain on the propellant surface. If the acceleration is less than the critical acceleration, $\mathrm{F}_{\mathrm{b}}<\mathrm{F}_{\mathrm{d}}$ and the particle will leave the surface. Thus we see that the critical acceleration is dependent on the velocity of the gas leaving the propellant surface. That is, the important parameter is not simply acceleration, but the ratio $F_{b} / F_{d}$. For a given propellant and particle size, $\mathrm{F}_{\mathrm{b}} / \mathrm{F}_{\mathrm{d}} \propto \mathrm{a} / \mathrm{U}_{\mathrm{g}}$. Assuming a perfect gas and the flame temperature to be independent of pressure,

$$
F_{b} / F_{d} \propto G P / r
$$

where

$$
\begin{aligned}
G & =a / g_{0} \\
g_{0} & =980 \mathrm{~cm} / \mathrm{sec}^{2} \\
P & =\text { pressure }
\end{aligned}
$$

The ratio $G P / r$ should be a correlating parameter if the critical particle size model is adequate. Data for burning rate augmentation as a function of acceleration and pressure plotted as $r / r_{0}$ versus $G P / r$ should lie on a single curve.

Referring to Figure 54, which shows burning rate augmentation versus acceleration for the $\times 304$ propellant, we see that the augmentation is lower at 1500 psia than at 1000 psia or 500 psia. Since increasing pressure by a factor of two results in a corresponding burning rate increase 
of less than a factor of two ${ }^{8}$, replotting the curves in Figure 54 as $r / r_{0}$ versus G P/r would move the 1500 psia curve farther away from the 500 and 1000 psia curves. Hence Crowe's model does not predict the observed dependence of burning rate augmentation on pressure.

The experimental results for the $\mathrm{X} 104$ propellant shown in Figure 34 suggest that the parameter G P/r may correlate the data. Figure 67 shows the X104 data at 500 and 1500 psia plotted as a function of $G \mathrm{P} / \mathrm{r}$. It was assumed that the burning rate augmentation could be described by

$$
r / r_{0}=1+\alpha(G P / r)^{n}
$$

The ordinate in Figure 67 is $\log \left(r / r_{0}-1\right)$ and the abscissa is $\log (\mathrm{G} P / r)$. The Figure, however, shows that $G P / r$ is not a correlating parameter for the X104 propellant.

The behavior of the non-aluminized X101 is particularly interesting. Since Crowe's theory is based on energy considerations, the location of the ignition wire should have no effect. However, we see that when the ignition wire was not intact at the end of the experiment, the non-aluminized propellant was affected by acceleration more than any of the aluminized propellants. Similarly, propellant extinction, which did occur with and without the ignition wire intact, is not predicted. The theoretical curves shown in Figure 3 bear the closest resemblance to the curves for the

${ }^{8}$ The pressure exponent $(n)$ for the $\mathrm{X} 304$ propellant is less than one. 


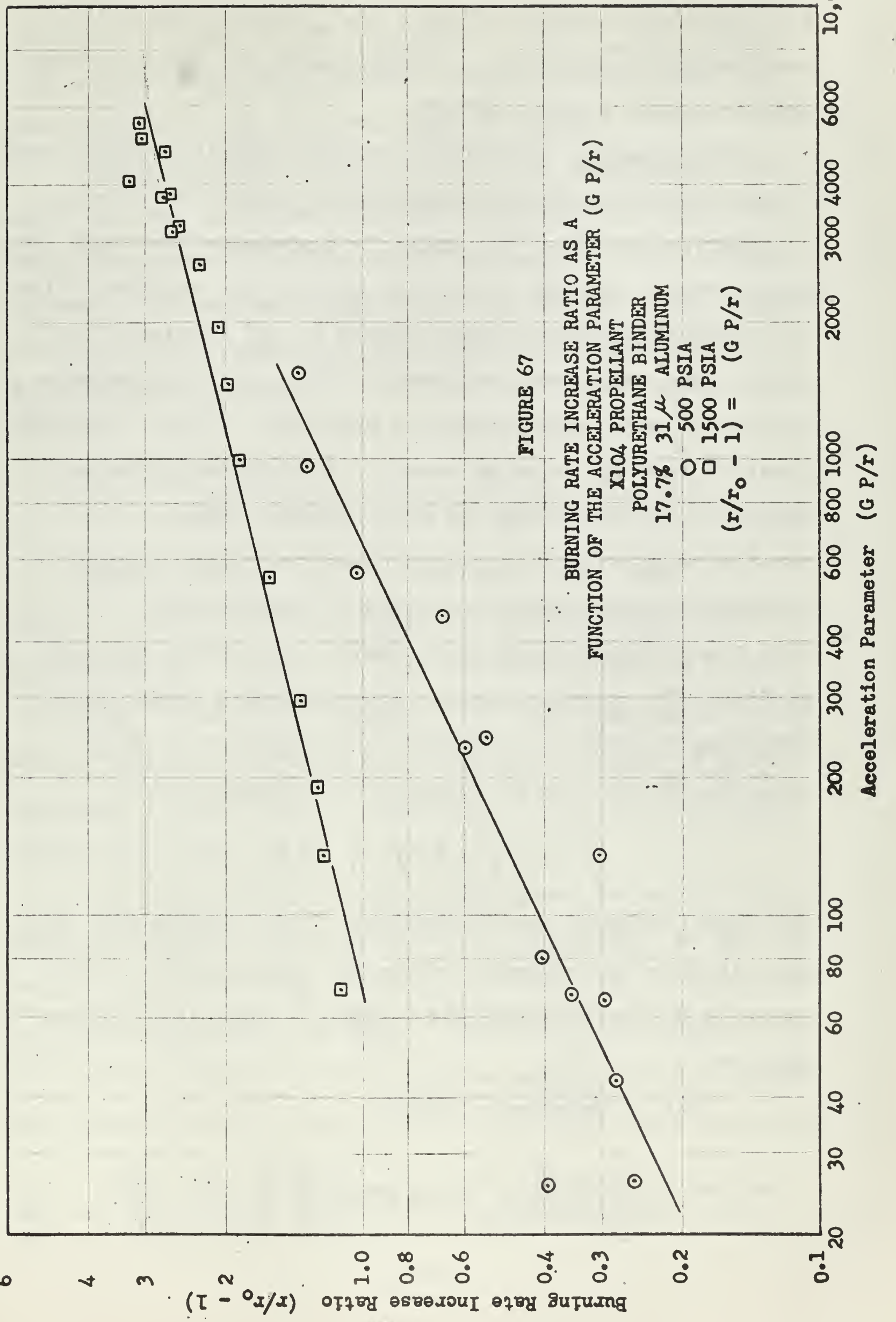


X301 propellant shown in Figure 42. However, the X301 is a non-aluminized propellant. According to the theory, acceleration should have no effect.

The foregoing comparisons between Crowe's critical particle size model and the experimental results of this investigation show that the model is inadequate for three reasons. First, the model fails to predict the observed effect of increasing aluminum mass loading at all accelerations. Second, the model does not predict the correct dependence of burning rate augmentation on pressure. Third, the model predicts that the burning rates of non-aluminized propellants will be unaffected by acceleration. However, the burning rates of both non-aluminized propellants investigated were significantly affected by acceleration.

The modified granular diffusion flame model proposed by Glick [7] is restricted to non-aluminized propellants. Referring to Equation (18) and the definition of $\phi$ on page 39 , we see that $\phi$ contains the unknown factor

$$
\left[d^{2}\left(\rho_{f} / \rho_{g}-\rho_{o} / \rho_{g}\right)\right] \equiv \psi
$$

The mass transport term in Equation (18) contains not only $\Psi$, but also the unknown fuel pocket dimension d. Introducing a new coefficient $\chi \equiv C_{1}(|\psi| d)^{\frac{1}{2}}$, Equation (19) may be written

$$
\begin{aligned}
r / r_{0} & =\frac{\psi a \rho_{g}^{2}}{32 \mu_{g} \rho_{s} r_{0}} \cos \theta+ \\
& {\left[\left(\frac{\psi a \rho_{g}^{2}}{32 \mu_{g} \rho_{s} r_{0}}\right)^{2} \cos ^{2} \theta+X\left(\frac{a \rho_{g}^{2}}{\mu_{g}^{2}}\right)^{\frac{1}{2}} s^{\frac{1}{2}}+1\right]^{\frac{1}{2}} }
\end{aligned}
$$


Equation (23) may be used to predict burning rate change with acceleration from two data points provided that the gas properties $\rho_{g}, \mu_{\mathrm{g}}$, and $\rho_{\mathrm{g}} \mathrm{D}$ are known.

Glick's theory is compared with the experimental results for the non-aluminized X301 propellant in Figure 68 . Using gas property values listed on page 40 , which are believed to be reasonable estimates for the $\times 301$ propellant at 1000 psia, and assuming $\psi$ independent of acceleration, Equation (23) becomes

$$
\begin{aligned}
r / r_{0}= & 2.44 \psi G+ \\
& {\left[(2.44 \psi G)^{2}+2.32 \times 10^{2} x G^{\frac{1}{2}}+1\right]^{\frac{1}{2}} }
\end{aligned}
$$

From Figure 39, $r / r_{0}=1.228$ at $G=300$, and $r / r_{0}=1.36$ at $G=1500$. Equation (24) fits these two points when $\psi=-0.49 \times 10^{-4} \mathrm{~cm}^{2}$ and $x=1.49 \times 10^{-4} \mathrm{~cm}^{3 / 2}$. The curve representing Equation (24) and these values of $\boldsymbol{\psi}$ and $x$ is shown in Figure 68. The Figure indicates good agreement between Glick's theory and the data at accelerations above $200 \mathrm{G}$.

It is important to notice that $\psi$ was found to be a negative number. Since $\psi \equiv d^{2}\left(\rho_{f} / \rho_{g}-\rho_{0} / \rho_{g}\right)$ we are led to the conclusion that the density of the oxidizer gas must be greater than the fuel gas. ${ }^{9}$ Hence with acceleration perpendicular to and toward the burning surface the fuel pocket moves away from the surface faster than without acceleration.

9 The products of binder and oxidizer decomposition are not sufficiently well known to argue strongly for or against this result. 


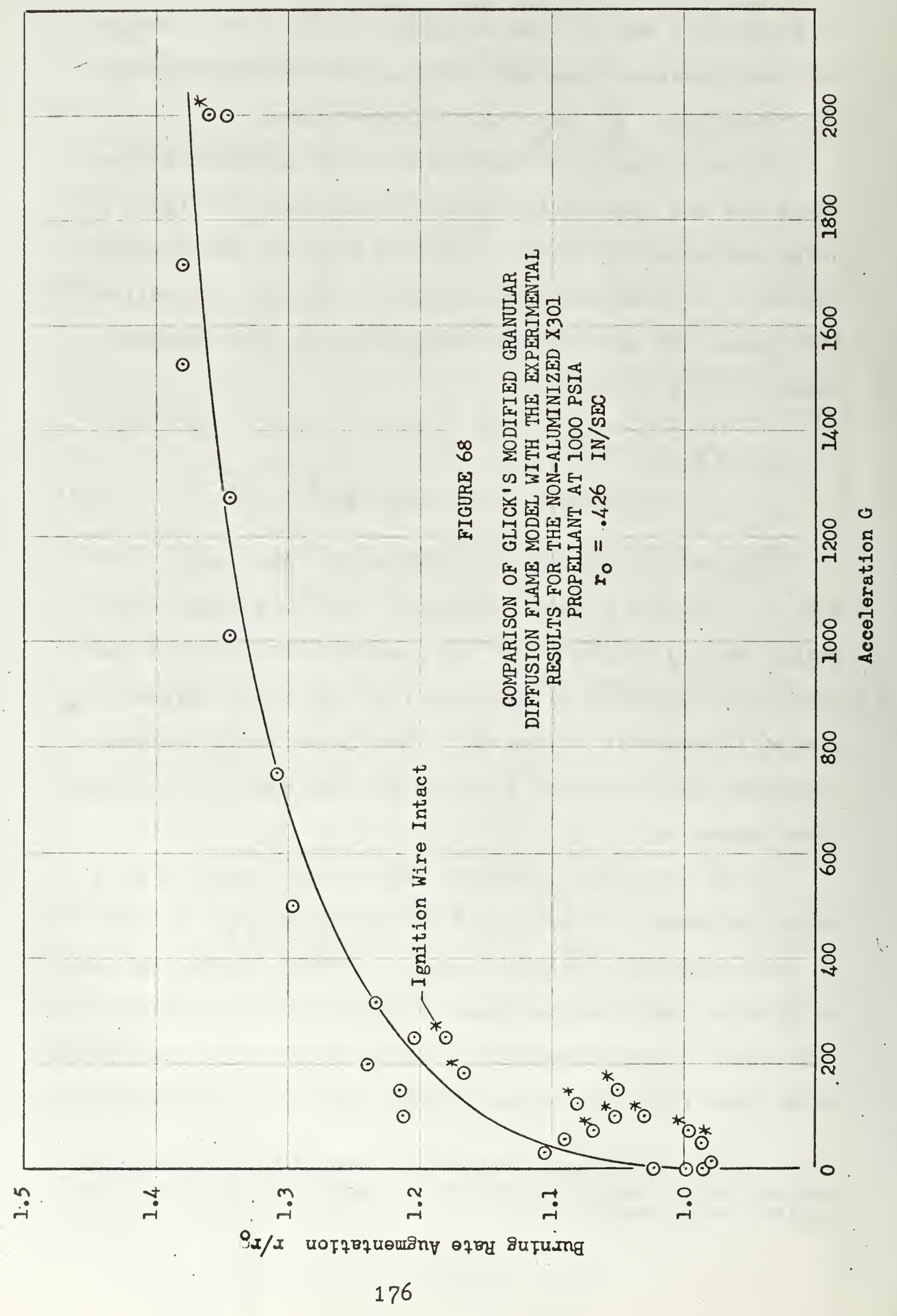


If the acceleration vector were perpendicular to and away from the propellant surface, the fuel pocket should move slower than without acceleration. Thus Glick's model predicts that the $\mathrm{X} 301$ propellant will be affected more by acceleration away from the burning surface than by acceleration toward the burning surface.

Glick's theory also predicts an immediate increase in burning rate as acceleration is increased from zero. However, the experimental results indicate that the burning rate of the $X 301$ propellant is independent of acceleration up to about $100 \mathrm{G}$ when the ignition wire remains intact. Moreover, the theory predicts that burning rate augmentation at a given acceleration should increase with increasing pressure, whereas, the experimental results for the $\mathrm{X} 301$ propellant (Figure 42) show that increasing pressure caused the burning rate augmentation to decrease. We may conclude that although Glick's modified granular diffusion flame theory may be fitted to the $\mathrm{X} 301$ propellant data at 1000 psia provided that the two coefficients $\psi$ and $\mathcal{X}$ in Equation (24) are properly chosen, the predicted burning rate change at low acceleration and the predicted effect of a change in pressure level are not confirmed. These results cast doubt on the adequacy of the model. 


\section{SUMMARY AND DISCUSSION}

The burning rates of all propellants investigated were found to change with acceleration. The effect of acceleration depended on propellant ingredients and ranged from an increase in burning rate by a factor of four to propellant extinguishment immediately after ignition. The burning rates of the aluminized propellants increased with acceleration, and the burning rate augmentation was considerably greater for the slow burning propellant than for the two medium burning propellants. The slow burning polyurethane propellant had a uni-modal oxidizer and showed a maximum burning rate augmentation of 4 . The PBAN propellant with a bimodal oxidizer showed a maximum burning rate augmentation of 1.5 , and the carboxy-terminated polybutadiene propellant with a tri-modal oxidizer showed a maximum burning rate augmentation of only 1.2 . These results indicate that burning rate augmentation depends to a significant degree on binder composition and/or oxidizer particle size distribution. It further points out that slow burning aluminized propellants should be avoided in rocket motor applications involving acceleration perpendicular to and into the burning surface.

The burning rate augmentation for a given binder oxidizer system depended on aluminum mass loading and aluminum mass median particle size. The general effects of changing aluminum mass loading or particle size in one binder - oxidizer system were similar to the effects shown in another binder - oxidizer system. However, a change in 
aluminum mass loading or particle size did not produce the same relative change in burning rate at all acceleration levels. This suggests that the mechanism of the burning rate increase is not the same at all levels of acceleration. The two non-aluminized propellants, one with a PBAN binder and the other with a polyurethane binder, were each affected differently by acceleration. The PBAN propellant was insensitive to acceleration up to $100 \mathrm{G}$ and then displayed approximately the same burning rate augmentation as the aluminized PBAN propellants. In contrast, the polyurethane propellant showed an increase in burning rate at 100G, but as acceleration was increased, the burning rate decreased, and at 300G the strands stopped burning shortly after ignition. The cause for extinguishment may have been the formation of a film of binder residue which impeded the heat transfer from the gas-phase reaction.

The disposition of the ignition wire had a significant effect on the behavior of the non-aluminized propellants. In the PBAN propellant the effect was confined to accelerations up to 300G. When the wire was permitted to fall into the inhibitor case the burning rate increased rapidly as radial acceleration was increased from zero. Above $300 \mathrm{G}$ the disposition of the ignition wire had no effect. The nonaluminized polyurethane propellant was affected by the presence of the ignition wire at all accelerations. When the wire was permitted to fall into the case, the response of both the polyurethane and PBAN propellants was similar to 
the general behavior of the aluminized propellants. In addition, the degree of burning rate augmentation was about the same magnitude as that shown by the aluminized propellants. The disposition of the ignition wire had insignificant effect on the acceleration sensitivity of the aluminized propellants.

The burning rate augmentation at a given acceleration level was found to change with a change in pressure. In some instances the burning rate augmentation increased with increasing pressure, and in other instances the augmentation decreased. The burning rate pressure exponent $(n)$ of the slow burning polyurethane propellant was higher with acceleration than without. On the other hand, the pressure exponents of the two medium burning propellants were relatively unaffected by acceleration.

An aluminum oxide residue was found in the bottom of the aluminized propellant inhibitor cases, and the amount of oxide retained increased as the acceleration level increased. The oxide from burning rate experiments at $400 \mathrm{G}$ and above could be removed as a solid block from the inhibitor cases, and at $1000 \mathrm{G}$ the mass of aluminum in the oxide block was over one-half the total mass of aluminum which had been contained in the propellant strand.

The two models which have been proposed by other investigators $[6,7]$ to predict the effect of acceleration on propellant burning rate do not adequately predict the behavior of the propellants used in this investigation. 
Crowe's critical particle size model [6] predicts that acceleration will affect the burning rate of aluminized propellants only. This was not borne out in the present investigation. In addition, the predicted effects of changing aluminum mass loading and pressure were not confirmed. Glick's modified granular diffusion flame model [?] predicts that the burning rates of non-aluminized propellants will increase with acceleration. Good agreement was found between the theory and the data for the non-aluminized PBAN propellant between 200 and 2000G. A consequence of fitting the theory to the data was to show that the oxidizer gas must be more dense than the fuel gas. However, Glick's model predicts dependence of burning rate augmentation on pressure in a manner opposite to that observed, and it predicts an immediate increase in burning rate as acceleration is increased from zero while the experimental results indicate little or no change in burning rate up to $100 \mathrm{G}$.

The modified granular diffusion flame model leads to an expression for burning rate augmentation which can be written

$$
r / r_{0}=\phi+\left[\phi^{2}+K G^{\frac{1}{2}}+1\right]^{\frac{1}{2}}
$$

where $\phi$ is a relative motion term and the term $\mathrm{KG}^{\frac{1}{2}}$ accounts for convective mass transport. It can be shown that $|\phi| \ll K G^{\frac{1}{2}}$ when $\left|\rho_{\mathrm{f}} / \rho_{\mathrm{g}}-\rho_{\mathrm{O}} / \rho_{\mathrm{g}}\right| \leq 1$ and $\mathrm{d} \leq 20$ microns. This suggests a simplified form in which the relative motion term $\Phi$ is ignored. The expression for $r / r_{0}$ is then

$$
r / r_{0}=(1+K G)^{\frac{1}{2}}
$$


The exponent ( $n$ ) was found from the data in Figure 69 by plotting $\log \left(r^{2} / r_{0}{ }^{2}-1\right)$ versus $\log G$. A straight line with slope 0.25 proved to be a reasonable fit to the data in the acceleration range of 300 to 2000G. Equation (25) then becomes

$$
r / r_{0}=\left(1+K G^{\frac{1}{4}}\right)^{\frac{1}{2}}
$$

The coefficient $K$ was found to be 0.137 by fitting Equation (26) to the point $r / r_{0}=1.36$ at $1500 G$. This solution is shown in Figure 69. A comparison with the data shows good agreement from 300 to $2000 G$.

The experimental results for the X301 propellant suggest the existence of a critical acceleration below which the propellant burning rate is unaffected. As acceleration is increased above the critical value the burning rate increases and appears to approach a limit. Diffusion flame experiments using gas jets in air and a one $G$ acceleration field indicate that the flame remains laminar with gas jet velocities up to $2000 \mathrm{~cm} / \mathrm{sec}$. [14] Since the velocity of the gas leaving a burning propellant surface is typically of the order $100 \mathrm{~cm} / \mathrm{sec}$, one might conclude that transition to a turbulent flame would not occur. However, the body forces resulting from large accelerations and a difference in density between the fuel gas and oxidizer gas would tend to promote flow instability at the boundaries between fuel rich and oxidizer rich regions. This suggests a modified form of Equation (25) in which the acceleration $G$ is replaced by the difference between the actual acceleration and some critical 


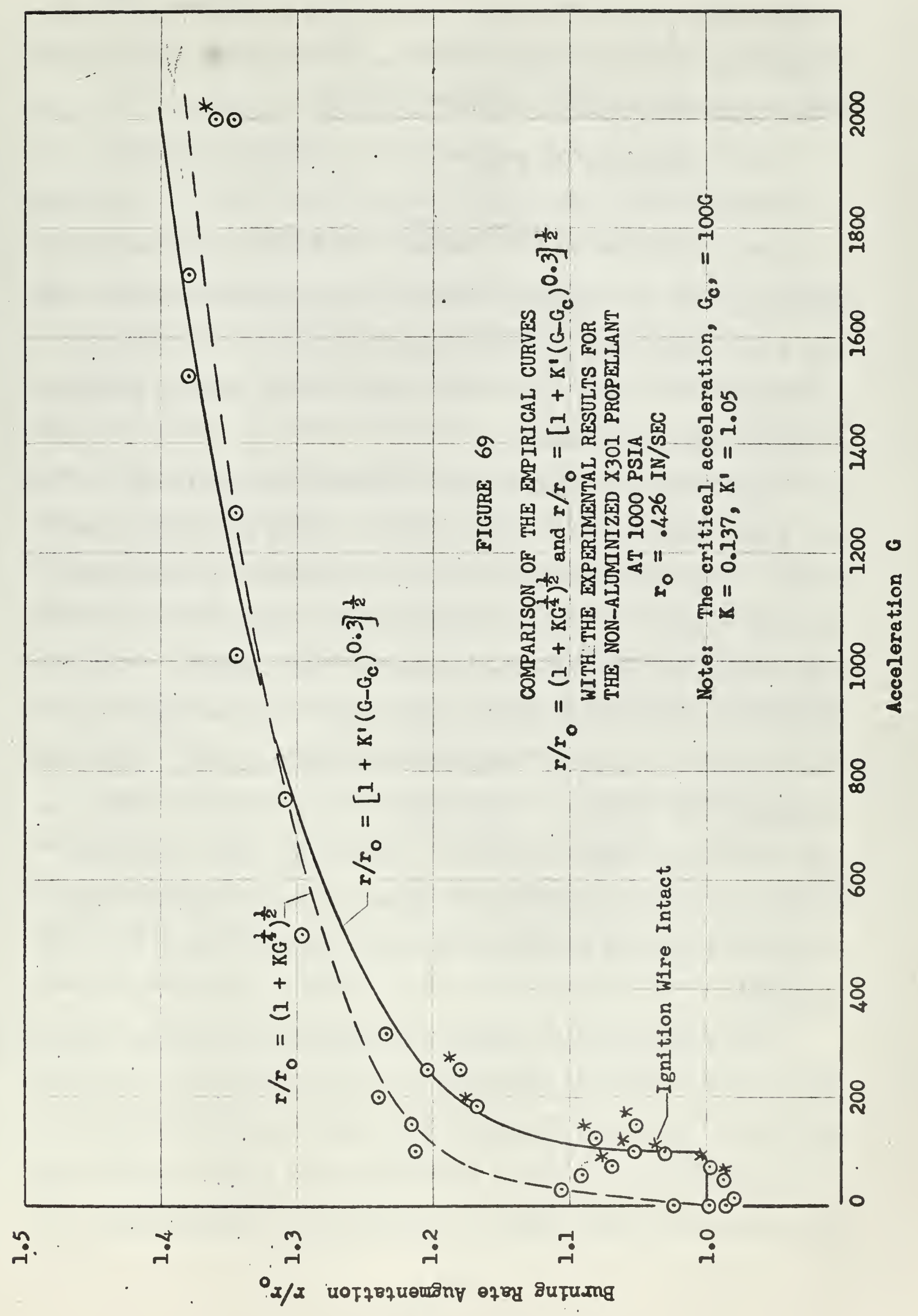


acceleration $G_{c}$ below which the propellant burning rate is the same as without acceleration. In this case, the burning rate augmentation might be described by

$$
\begin{aligned}
& r / r_{0}=1, \quad G \leq G_{c} \\
& r / r_{0}=\left[1+K^{\prime}\left(G-G_{c}\right)^{n}\right]^{\frac{1}{2}}, \quad G \geq G_{c}
\end{aligned}
$$

Equation (27) is compared with the experimental results for the X301 propellant at 1000 psia in Figure 69. The curve, for which $K^{\prime}=1.05, G_{c}=100$, and $n=0.3$, agrees reasonably well with the data.

The observed burning rate increase is attributed to the gas phase reaction occurring closer to the propellant surface. It is believed that in a sufficiently strong acceleration field, the body forces promote increased mixing of the originally separate oxidizer and fuel species. With a propellant containing relatively small oxidizer particles the original degree of unmixedness would be small, thus decreasing the relative importance of body forces. Hence, a non-aluminized PBAN propellant containing large oxidizer crystals would be expected to show a greater burning rate augmentation with acceleration than one with small oxidizer crystals.

The burning rate augmentation shown by the non-aluminized propellants when the nichrome ignition wire was allowed to fall into the inhibitor case was essentially the same as the augmentation shown by the aluminized propellants. This indicates that the important mechanism of burning rate 
augmentation in the aluminized propellants is conduction. In the case of the non-aluminized propellant the nichrome wire is believed to have been in one or more pieces, possibly spherical in shape, lying on the burning propellant surface. In this position they would have contributed to increased localized heat transfer. Thus small pits would have been formed with an attendant increase in burning area. Increased localized heat transfer at discrete locations may be the predominant mechanism of the burning rate increase shown by aluminized propellants at low acceleration. The aluminum agglomerates may collect in small pools. Pools of both reacting aluminum and completely reacted oxide would be good conductors of energy to the propellant surface. These pools would gradually settle into pits formed by the more rapid pyrolysis of binder and oxidizer. Thus the localized burning rate increase would be augmented by an increase in burning surface area.

On the basis of this hypothesis it would appear that one approach to reducing burning rate augmentation at low accelerations would be to somehow reduce the aluminum agglomerate size. Crump's film [9] indicates that agglomerate size decreases with smaller oxidizer crystal size. Hence in those applications where a medium to high burning rate propellant could be used, acceleration effects might be adequately controlled through the use of small oxidizer particle sizes. A more basic approach would be to prevent the accumulation of aluminum particles which leads to 
agglomeration. It might be speculated that the aluminum particles are cemented together by partially decomposed binder residue as they emerge from the regressing binder and oxidizer. In that case, coating the aluminum particles with a substance to which the binder residue would not adhere, or elimination of the residue altogether would enable the aluminum particles to leave the surface individually.

At high accelerations there is sufficient oxide retained to cover the burning surface with a molten layer. Hence independent pools causing increased localized heat transfer probably do not exist. Instead, the oxide may be expected to contact the burning surface in a random fashion as the oxide is violently stirred by the gaseous products of combustion flowing through the oxide mass. In this case, the initial aluminum particle size would not be expected to affect the depth of the oxide layer. However, initial aluminum particle size . aluminum mass loading, and the dispersion of the aluminum particles in the binder may affect the thermal conductivity at the propellant - oxide inteirface.

The burning rate of the non-aluminized PBAN propellant increased significantly at high acceleration even though the ignition wire remained intact. Hence the burning rate augmentation shown by the aluminized PBAN propellants at high acceleration cannot be attributed solely to the presence of the aluminum. It is likely that coupling between two or more mechanisms is involved. 
An adequate analytical model describing the effect of acceleration on the burning rate of non-aluminized composite propellants must account for the effects of changing pressure level, oxidizer particle size distribution, and binder composition. A model for aluminized composite propellants must, in addition to the above variables, account for changing aluminum mass loading and aluminum mass median particle size. At this time the mechanisms by which the above parameters affect propellant burning rate in a one $G$ acceleration field are not well understood. Several models have been formulated in an attempt to explain the change of burning rate with pressure, but no one model has gained universal acceptance. Due to the complex nature of the burning mechanism, the formulation of a mathematical model to account for the effect of acceleration on propellant burning rate does not appear to be feasible in the light of present knowledge. An acceptable mathematical model is unlikely to evolve until more of the fundamental mechanisms have been. extensively investigated and are understood.

\section{Recommendations for Future Work}

The results reported here indicate that further investigations are required to gain a better understanding of the mechanisms involved in burning rate augmentation associated with acceleration. High-speed photography of propellants burning in acceleration fields would be a significant contribution. Such a study could provide important information concerning the formation and structure of the 
aluminum oxide mass which has been found in the bottom of the inhibitor cases.

The relative importance of oxidizer particle size and binder composition on the burning rate augmentation of aluminized propellants could be studied by using the same two uni-modal oxidizer particle size distributions, one with large particles and one with small particles, in propellants with two different binders. The same aluminum mass loading and particle size distribution should be used in all four propellants. The use of aluminum particle size distributions with small variance but different mass median diameters would help to more clearly define the importance of oxidizer particle size at low accelerations where agglomeration is believed to be important.

The retention of aluminum oxide in the motor combustion chamber is a significant factor in the performance losses experienced with spinning rocket motors. An expression for the mass of oxide retained as a function of propellant burning rate, aluminum mass loading, acceleration and propellant grain web thickness would be of value to the rocket motor designer. Experimental data could be obtained by burning propellant strands similar to those used in the present investigation, but with various lengths.

The non-aluminized propellants merit further investigation. Experiments with the acceleration vector at various angles to the burning surface, including parallel to and away from, may indicate possible reasons for propellant 
extinction in one instance and burning rate increase in another. Additional evidence to evaluate the basic concepts of the modified granular diffusion flame model proposed by Glick could be provided by two experiments. First, experiments similar to those reported herein should be conducted at low pressures where chemical reaction rates are believed to be rate controlling. Second, propellants with different oxidizer crystal size should be investigated.

Initial propellant temperature may be an important parameter in burning rate augmentation due to acceleration. Experiments at constant acceleration and constant pressure but with varying initial propellant temperature may show that propellant burning rate temperature coefficients are affected to a significant degree by acceleration. 
CHAPTER V

CONCLUSIONS

The work reported here is believed to be the first systematic investigation of composite propellant burning rates at high acceleration. Previously reported investigations have been limited to one propellant and 300G, or spinning motor experiments with radial accelerations up to about $100 G$.

Acceleration perpendicular to and into the burning surface was found to affect the burning rates of both aluminized and non-aluminized composite propellants. Prior to this investigation it was generally believed that acceleration had no effect on the burning rate of non-aluminized composite propellants.

The primary factor affecting the relative amount of burning rate increase with acceleration was found to be oxidizer particle size distribution and/or binder composition. Acceleration effects may be reduced by using an oxidizer with small mass median particle size. The pressure level was found to be a secondary factor. The relative burning rate increase was greater with increasing pressure for some propellants and less for others. Aluminum mass loading and mass median particle size are also important, but the 
significance of a change in either of these two parameters depends on the acceleration level.

The presence of a small piece of nichrome wire at the burning surface of a non-aluminized composite propellant results in a relative burning rate increase with acceleration quite similar to that of the same propellant with an aluminum fuel additive.

The burning rate pressure exponent $(n)$ increases with acceleration for some propellants but remains essentially unchanged for other.

No large scale time dependence is indicated by average burning rates over adjacent one-half inch intervals in two and one-quarter inch long propellant strands.

The models proposed by Crowe [6] and GIick [7] do not adequately predict the effect on relative burning rate increase with acceleration of a change in aluminum mass loading or pressure. Moreover, other simplified models do not suggest themselves in the light of present knowledge.

Further investigations, recommended in Chapter IV, are required to gain a deeper insight into the mechanisms involved. 
1. Vecchio, R., and Harnett, S., Static Spin Testing of Solid Propellant Rockets, Picatinny Arsenal, Dover, New Jersey, (CPIA Publication No. 24, Bulletin of the 1st Meeting ICRPG Working Group on Static Testing), oct. 1963

2. Swain, Robert L., Lucy, Melvin H., and Foss, Peter H., Rocket Motor Spin Test Apparatus, NASA Langley Research Center, Langley Station, Hampton, Va., (Presented at the Second Annual Meeting of the ICRPG Working Group on Static Testing, Redlands, Calif., Oct 21-23, 1964)

3. Lucy, M.H., Swain, R.L., and Hudson, J.H. Jr., Variable Dynamic Force Vector Rocket Test Apparatus, NASA-LRC, Hampton, Va., (Paper presented at the 2nd Annual Meeting of the ICRPG Working Group on Static Testing, Redlands, California, oct 21-23, 1964)

4. Redel, Inc., An Investigation of the Effects of Acceleration on the Burning Characteristics of Solid Propellants (U), Redel, Inc., 2300 E. Katella Ave., Anaheim, Calif., Contract No. AF33(616)6779 Report No. KPR-5, Feb. 1961 (CONFIDENTIAL)

5. Northam, G.B., An Experimental Investigation of the Effects of Acceleration on the Combustion Characteristics of an Aluminized Composite Solid Propellant, MS Thesis, Virginia Polytechnic Institute, June, 1965

6. Crowe, C.T., Dunlap, R., Hermsen, R., et al., Investigation of Particle Growth and Ballistic Effects on Solid Propellant Rockets, (UTC-2128-QT3), United Technology Center, Division United Aircraft Corporation, Sunnyvale, Calif., (BuWeps Contract No. NOw 65-0222f) Oct. 1965

7. Glick, R.L., An Analytical Study of the Effects of Radial Acceleration Upon the Combustion Mechanism of Solid Propellant, Bi-Monthly Status Report No. 2, Report No.306-66, Contract NAS 7-406, Thiokol Chemical Corp., Huntsville, Alabama, Jan. 1966

8. Private Communication, Dr. C.T. Crowe, United Technology Center, Sunnyvale, California, April, 1966 
9. Crump, J.E., Aluminum Combustion in Composite Propellants, Proceedings of the 2nd ICRPG Combustion Conference, Aerospace Corp., El Segundo, Calif., Nov. $1-5,1965$

10. Summerfield, M., et al., Burning Mechanism of Ammonium Perchlorate Propellants, Progress in Astronautics and Rocketry, Vol. I., p. 142-182, Academic Press, Inc., New York, New York, 1960

11. Houghton, G., and Redfield, J., Mass Transfer and Drag Coefficients for Single Bubbles at Reynolds Numbers of $0.02-5000$, Chemical Engineering Science, Vol. 20, p. 131-139, 1965

12. Cinémicrographie de la Combustion des Poudres Composites Métallisées (1964), (Cinemicrography of the Combustion of Composite Solid Propellants Containing Metal Additives), Office National d'Etudes et de Recherches Aérospatiales, 29, Avenue de la Division Leclerc, Chatillon-Sous-Bagneux, (Seine) France

13. Index to the X-RAY POWDER DATA FILE (1960), ASTM Special Technical Publication 48-I, American Society for Testing Materials, 1916 Race Street, Philadelphia 3, Pennsylvania

14. Penner, S.S., Chemistry Problems in Jet Propulsion, Pergamon Press, New York, 1957, p. 270 
Whittaker, A.G., An Hypothesis Concerning the Action of Catalysts in Mesa Propellants, and the Effect of Acceleration on Propellant Consumption Rate, U.S. Naval Ordnance Test Station, China Lake, Calif., Memorandum Report IDP-8, March, 1955

Hercules Powder Co., Allegany Ballistics Laboratory, Cumberland, Md:. Monthly Progress Report No. 47, Contract NOrd 10431, September, 1955, (CONFIDENTIAL)

Redel, Inc., An Investigation of the Effects of Accelera$\therefore$ tion on the Burning Characteristics of Solid Propellants (U), Redel, Inc., $2300 \mathrm{E}$. Katella Ave., Anaheim, Calif., Contract No. AF33(616)6779

$\begin{array}{llll}\text { Report No. } & \text { KPR-1 } & \text { Feb. } 1960 & \text { (CONFIDENTIAL) } \\ \text { KPR-2 } & \text { May } 1960 & \text { (CONFIDENTIAL) } \\ \text { KPR-3 } & \text { Aug. } 1960 & \text { (CONFIDENTIAL) } \\ \text { KPR-4 } & \text { Nov. } 1960 & \text { (CONFIDENTIAL) }\end{array}$

Eirich, M.D., Acceleration Effects on Propellant Burning (U), Allegany Ballistics Laboratory, Cumberland, Md., ABL/EPA-8, Contract NOrd 16640, October, 1962 (CONFIDENTIAL)

Arthur D. Little, Inc., Cambridge, Mass., Design Studies on a $105 \mathrm{~mm}$ Gun-Boosted Rocket (U), Final Report to Commanding Officer, Picatinny Arsenal, Dover, New Jersey, Contract No. DA-19-020-ORD-5695, January, 1963, (CONFIDENTIAL)

Redel, Inc., An Investigation to Characterize the Effects of Acceleration on the Burning of a Gun-Boosted Rocket Propellant (U), Redel, Inc., 2300 E. Katella Ave., Anaheim, Calif., Report No. VML-6, First Phase: Final Report, May, 1963, (CONFIDENTIAL)

Thiokol Chemical Corp., Evaluation of Solid Rocket Motor Ballistic Properties Under High 'G' Environment, Vol. I, Thiokol Chemical Corp., Elkton, Md., Final Report, RTD-TDR-63-1054, June, 1963 
Guthrie, W.D., and Chen, E.C.M., Effects of Acceleration on Burning Rates of Solid Propellants (U), U.S. Army Missile Command, Redstone Arsenal, Alabama, Report No. RK-TR-63-22, October, 1963, (CONFIDENTIAL)

Meraz, D., Jr., Iiterature Survey of Spin Stabilized Rockets and Rocket Assisted Projectiles (U), Propulsion Development Department, U.S. Naval Ordnance Test Station, China Lake, Calif., NAVWEPS REPORT 8489, January, 1964, (CONFIDENTIAI)

Redel, Inc., An Investigation to Characterize the Effects of Acceleration on the Burning of Gun-Boosted Rocket Propellant (U), Redel, Inc., 2300 E. Katella Ave., Anaheim, Calif., Report No. VMI-14, Second Phase: Final Report, March, 1964, (CONFIDENTIAL)

White, D.W., and Nelius, M.A., Results of Testing Two HPC-ABI $\mathrm{X}-258$ ( $\mathrm{S} / \mathrm{N}^{\prime} \mathrm{s} \mathrm{RH}-56$ and $\overline{\mathrm{RH}}-58$ ) Solid Propellant Rocket Motors Under the Combined Effects of Simulated Altitude and Rotational Spin, ARO, Inc., Arnold Engiheering Development Center, Arnold Air Force Station, Tenn., AEDC-TDR-64-97, May, 1964

Nelius, M.A., Results of Testing Two NOTS 100B Spherical Rocket Motors Under the Combined Effects of Simulated Altitude and Rotational Spin (U), ARO, Inc., AEDC, Arnold Air Force Station, Tenn., Report No. AEDC-TDR64-102, May, 1964, (CONFIDENTIAL)

Redel, Inc., An Investigation to Characterize the Effects of Acceleration on the Burning of Gun-Boosted Rocket Propellant (U), Redel, Inc., 2300 E. Katella Ave., Anahe im, Calif., Report No. VML-21, Project 5118, Final Report, December, 1964, (CONFIDENTIAI)

Propulsion Development Dept., NOTS, China Lake, Symposium (on) the Behavior of Propellants Under Acceleration Fields (U), U.S. Naval Ordnance Test Station, China Lake, Calif., June, 1965, (CONFIDENTIAL)

DeDapper, J.W., and Drobot, W., The Effect of Acceleration on The Burning Rates of Selected Propellants (U), Classified Papers, AIAA Meeting, Wash., D.C., February 1-3, 1965, (CPIA Publication No. 83, August, 1965) (CONFIDENTIAL) 
Glick, R.L., An Analytical Study of the Effects of Radial Acceleration upon the Combustion Mechanism of Solid Propellant, Thiokol Chemical Corp., Huntsville, Ala. Bi-Monthly Status Report No. 1, October, 1965

Bi-Monthly Status Report No. 3, March, 1966 
APPENDIX I

DERIVATION OF AN EXPRESSION FOR

$\left(\rho_{\mathrm{f}} / \rho_{\mathrm{g}}-\rho_{\mathrm{o}} / \rho_{\mathrm{g}}\right)$ IN TERMS OF

GAS MOLECULAR WEIGHTS AND MASS FRACTIONS

The unknown term $\left(\rho_{\mathrm{f}} / \rho_{\mathrm{g}}-\rho_{\mathrm{O}} / \rho_{\mathrm{g}}\right)$ appears in the modified granular diffusion flame model proposed by GIick. [7] The magnitude of this term is dependent upon the molecular weights and mass fractions of the fuel and oxidizer gases. It is also dependent upon the temperatures of the two components. The derivation presented here is based on the following assumptions:

1. The fuel and oxidizer gases are not mixed.

2. The fuel gas is in pockets of dimension d surrounded by oxidizer gas.

3. Both fuel and oxidizer gases are at the same temperature and pressure.

4. Both components behave as perfect gases. The symbols to be used are as follows:

$\mathrm{W}=$ molecular weight

$X=$ mol fraction

$Y=$ mass fraction

$f=$ subscript denoting fuel gas

o = subscript denoting oxidizer gas

$g$ = subscript denoting average of fuel and oxidizer gases

$P=$ pressure 


$$
\begin{aligned}
\rho & =\text { density } \\
\mathrm{R} & =\text { universal gas constant } \\
\mathrm{T} & =\text { temperature }
\end{aligned}
$$

The pressure of both components is the same, so that

$$
P=\frac{\rho_{f} R T}{W_{f}}=\frac{\rho_{0} R T}{W_{0}}=\frac{\rho_{g} R T}{W_{g}}
$$

and

$$
\frac{\rho_{f}}{W_{f}}=\frac{\rho_{o}}{W_{o}}=\frac{\rho_{g}}{W_{g}}
$$

Then

$$
\left(\rho_{f} / \rho_{g}-\rho_{o} / \rho_{g}\right)=\frac{W_{f}}{W_{g}}-\frac{W_{o}}{W_{g}}
$$

The average molecular weight $\mathrm{W}_{g}$ is

$$
\mathrm{W}_{\mathrm{g}}=\mathrm{X}_{\mathrm{f}} \mathrm{W}_{\mathrm{f}}+\mathrm{X}_{\mathrm{o}} \mathrm{W}_{\mathrm{o}}
$$

where the mol fractions $X$ are

$$
X_{f}=\frac{Y_{f} / W_{f}}{Y_{f} / W_{f}+Y_{0} / W_{0}}, \quad X_{0}=\frac{Y_{0} / W_{0}}{Y_{f} / W_{f}+Y_{0} / W_{o}}
$$

Combining Equations (2) and (3), and recognizing that $Y_{f}+Y_{0}=1$, we get

$$
W_{g}=\frac{1}{Y_{f} / W_{f}+Y_{o} / W_{o}}
$$

Substituting Equation (4) into Equation (1),

$$
\begin{aligned}
\left(\rho_{f} / \rho_{g}-\rho_{0} / \rho_{g}\right) & =\left(W_{f}-W_{0}\right)\left(Y_{f} / W_{f}+Y_{0} / W_{0}\right) \\
& =Y_{f}\left(1-W_{0} / W_{f}\right)+Y_{0}\left(W_{f} / W_{0}-1\right)
\end{aligned}
$$


Assume the solid propellant be $20 \%$ binder and $80 \%$ oxidizer, then $Y_{f}=0.2$ and $Y_{0}=0.8$. If we define $\eta \equiv W_{0} / W_{f}$ and assume $\left(\rho_{\mathrm{f}} / \rho_{\mathrm{g}}-\rho_{\mathrm{o}} / \rho_{\mathrm{g}}\right)=1$, Equation $(5)$ becomes

$$
1=0.2(1-\eta)+0.8(1 / \eta-1)
$$

The solution to Equation (6) is

$$
\eta=-8.47 \text { or } 0.47
$$

$W_{f}>0$ and $W_{0}>0$, hence $\eta=0.47 \simeq \frac{1}{2}$, or $W_{f} \simeq 2 W_{0}$. 
With the centrifuge in motion, gas confined in the surge tanks, bomb, and connecting tubing will tend to move outward due to centrifugal force. Hence the pressure in the combustion bomb will be higher than the pressure to which the system was charged while at rest. With the centrifuge in motion there will be pressure gradients throughout the field. The problem becomes more tractable, however, if:

1) the gas in the bomb is considered to be at the same pressure throughout and its mass concentrated at a point, 2) the gas in the two surge tanks is considered to be at the same pressure throughout and its mass concentrated at a point, 3) the two concentrated masses lie on the same radial and are connected by a thin tube of negligible volume.

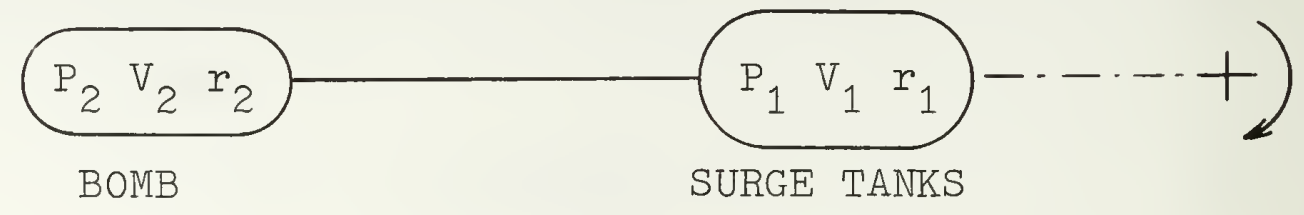

let $\mathrm{P}=$ pressure

$\mathrm{V}=$ volume

$r=$ radius

$A=$ cross sectional area of connecting tube

$\rho=$ gas density

$\omega=$ angular velocity $1 / \mathrm{sec}$ 


$$
\begin{array}{rlrl}
\mathrm{R}=\text { gas constant for } \mathrm{N}_{2} & =55.2 \mathrm{ft} \mathrm{lb} / \mathrm{lb} \mathrm{m} \\
\mathrm{T}=\text { gas temperature } & & =528^{\circ} \mathrm{R} \\
\mathrm{r}_{1}=\text { tank radius } & =8 \mathrm{in} . \\
\mathrm{r}_{2}=\text { bomb radius } & =35 \mathrm{in} . \\
\mathrm{g}=32.2 \mathrm{ft} / \mathrm{sec}^{2} & & \\
\mathrm{~V}_{1}=\text { tank volume } & & 1450 \mathrm{cu} . \mathrm{in} . \\
\mathrm{V}_{2}=\text { bomb volume } & =115 \mathrm{cu} . \mathrm{in} . \\
\mathrm{P}_{0}=\text { pressure in system with } \mathrm{w}=0
\end{array}
$$

In the connecting tube, where the centrifugal force must be balanced by a pressure gradient.

$$
\left(r \omega^{2}\right)(\rho \text { Adr })=A d P
$$

Assuming a perfect gas,

$$
\rho=P / R T
$$

Combining (2) and (1) gives

$$
\mathrm{dP} / \mathrm{P}=\left(\omega^{2} / 2 \mathrm{gRT}\right) 2 \mathrm{rdr}
$$

Assuming $T$ to be constant, and integrating between stations 1 and 2, (3) becomes

$$
\begin{aligned}
\mathrm{P}_{2} / \mathrm{P}_{1} & =\exp \left(r_{2}^{2}\left(1-r_{1}^{2} / r_{2}^{2}\right) \omega^{2} / g 2 \mathrm{RT}\right) \\
& =\exp \left(K \omega^{2} / g\right)
\end{aligned}
$$

where $K=r_{2}^{2}\left(1-r_{1}^{2} / r_{2}^{2}\right) / 2 R T=1.380 \times 10^{-4}$ ft $1 b_{m} / 1 b$.

Conservation of mass, when combined with (2) gives

$$
P_{\circ}\left(V_{1}+V_{2}+A\left(r_{2}-r_{1}\right)\right)=P_{1} V_{1}+P_{2} V_{2}+\int_{r_{1}}^{r_{2}} A P d r
$$


Neglecting the small amount of gas in the connecting tube, and rearranging (5) gives

$$
\mathrm{P}_{2} / \mathrm{P}_{0}=\left(\mathrm{V}_{2}+\mathrm{V}_{1}\right) /\left(\mathrm{V}_{2}+\mathrm{V}_{1} \mathrm{P}_{1} / \mathrm{P}_{2}\right)
$$

or

$$
\mathrm{P}_{2} / \mathrm{P}_{0}=1 /\left[1-\left(1-\mathrm{P}_{1} / \mathrm{P}_{2}\right) /\left(1+\mathrm{V}_{2} / \mathrm{V}_{1}\right)\right]
$$

From $(4),\left(\mathrm{P}_{1} / \mathrm{P}_{2}\right)_{\min }=\left[\exp \left(-\mathrm{K} \omega^{2} / \mathrm{g}\right)\right]_{\min }$ which occurs at the maximum speed of $155 \mathrm{rad} / \mathrm{sec}$

$$
\left(K \omega^{2} / g\right)_{\max }=0.1032
$$

and

$$
\left(\mathrm{P}_{1} / \mathrm{P}_{2}\right)_{\min }=0.92
$$

Hence

$$
\left[\left(1-\mathrm{P}_{1} / \mathrm{P}_{2}\right) /\left(1-\mathrm{V}_{2} / \mathrm{V}_{1}\right)\right]_{\max }=0.0742
$$

and

$$
\mathrm{P}_{2} / \mathrm{P}_{0} \simeq 1+\left(1-\mathrm{P}_{1} / \mathrm{P}_{2}\right) /\left(1-\mathrm{V}_{2} / \mathrm{V}_{1}\right)
$$

Letting $\mathrm{P}_{2}=\mathrm{P}_{0}+\Delta \mathrm{P}$, and combining (4) with (8)

$$
\begin{aligned}
\triangle \mathrm{P} / \mathrm{P}_{0}=\left[1-\exp \left(-\mathrm{K} \omega^{2} / \mathrm{g}\right)\right] /\left(1+\mathrm{V}_{2} / \mathrm{V}_{1}\right) \\
\begin{aligned}
\exp \left(-\mathrm{K} \omega^{2} / \mathrm{g}\right) & =1-\mathrm{K} \omega^{2} / \mathrm{g}+\left(\mathrm{K} \omega^{2} / \mathrm{g}\right)^{2} / 2-\ldots \\
& \simeq 1-K \omega^{2} / g \text { since } \mathrm{K} \omega^{2} / g \leq 0.1032
\end{aligned}
\end{aligned}
$$

Hence

$$
\Delta \mathrm{P} / \mathrm{P}_{0} \simeq\left(\mathrm{K} \omega^{2} / \mathrm{g}\right) /\left(1+\mathrm{V}_{2} / \mathrm{V}_{1}\right)=1.284 \times 10^{-4} \mathrm{w}^{2} / \mathrm{g}
$$

The curves shown in Figure 70 were derived from Equation (10). 


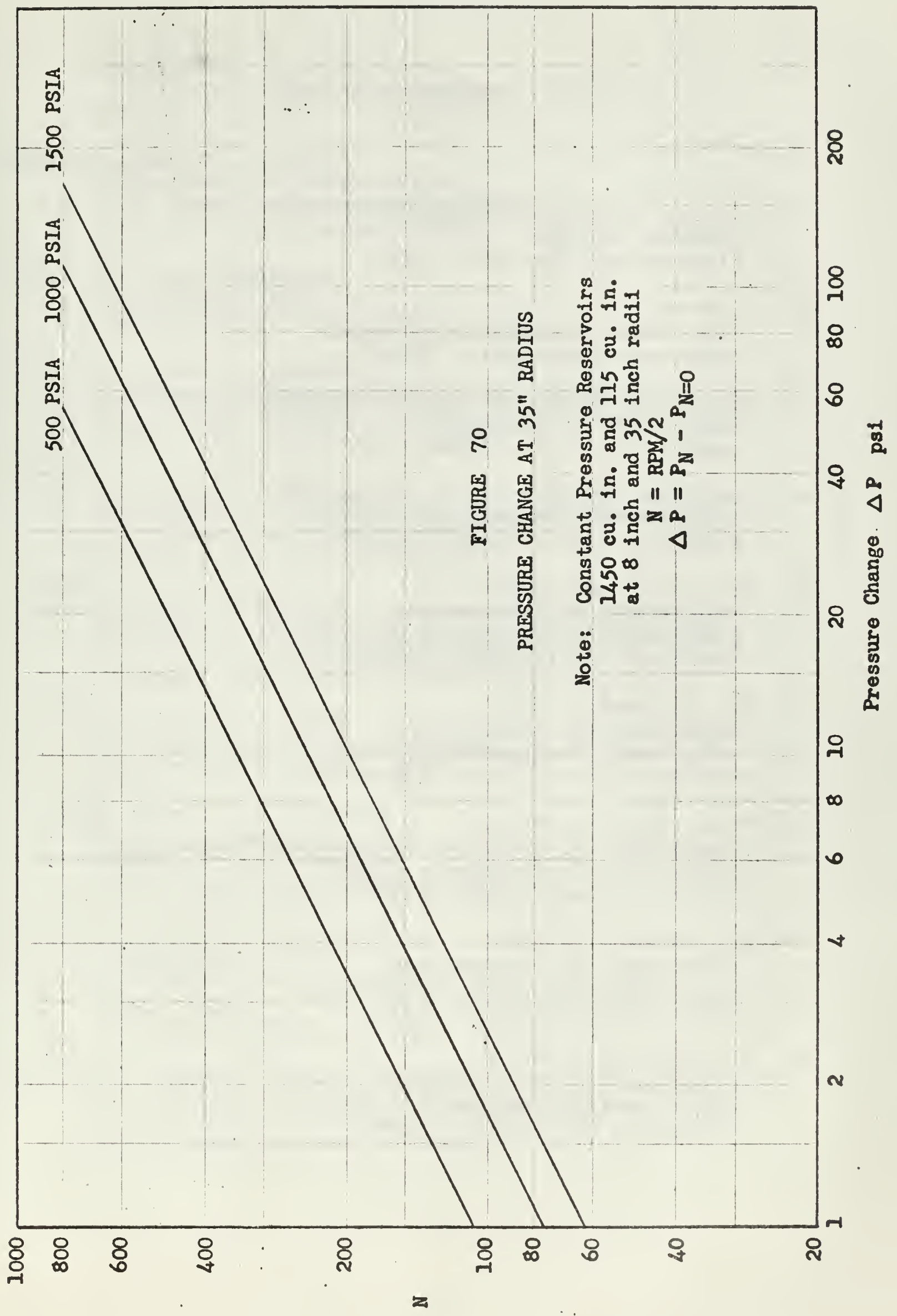


1. Defense Documentation Center

Cameron Station

Alexandria, Virginia 22314

2. Library

U.S. Naval Postgraduate School

Monterey, California 93940

3. Commander, Naval Air Systems Command

Department of the Navy

Washington, D.C. 20360

4. Chairman, Department of Aeronautics

U.S. Naval Postgraduate School

Monterey, California 93940

5. Dr. Roy E. Reichenbach

Department of Aeronautics U.S. Naval Postgraduate School

Monterey, California 93940

6. Dr. Richard W. Bell

Department of Aeronautics

U.S. Naval Postgraduate School

Monterey, California 93940

7. Prof. Carl A. Hering

Department of Material Science and Chemistry U.S. Naval Postgraduate School

Monterey, California 93940

8. Dr. James A. Miller

Department of Aeronautics

U.S. Naval Postgraduate School

Monterey, California 93940

Dr. Elmo J. Stewart

Department of Mathematics

U.S. Naval Postgraduate School

Monterey, California 93940 
10. Dr. Michael H. Vavra

Department of Aeronautics

U.S. Naval Postgraduate School

Monterey, California 93940

11. Mr. Edward J. Smith

Department of Aeronautics

U.S. Naval Postgraduate School

Monterey, California 93940

12. Dr. Ralph Anderson

188 Whisman Road

Mountain View, California 94041

13. Mr. James P. Diebold

Development Engineering Branch (Code 4571)

1

U.S. Naval Ordnance Test Station

China Lake, California 93557

14. Dr. John R. Clark

Department of Material Science and Chemistry U.S. Naval Postgraduate School

Monterey, California 93940

15. Dr. John W. Schultz

Department of Material Science and Chemistry U.S. Naval Postgraduate School

Monterey, California 93940

16. Dr. Robert I. Glick

Thiokol Chemical Corporation

Huntsville, Alabama 35809

17. Mr. Newton H. Anderson

Box 10, Code 4113

Pacific Missile Range

Point Mugu, California 93041

18. Mr. William D. Guthrie

AMSMI-RKA

U.S. Army Missile Command

Bldg. 7120

Redstone Arsenal, Alabama 35809

19. ICDR James B. Anderson, USN

Operational Test and Evaluation Force, Pacific

U.S. Naval Air Station, North Island

San Diego, California 92135 



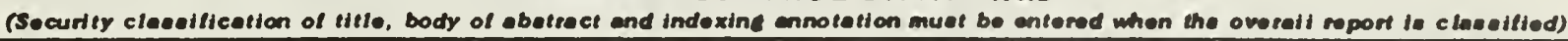
1. ORIGINATIN G ACTIVITY (Corporato euthor)

U.S. Naval Postgraduate School

Monterey, California 93940 20. REPORT SECURITY CLASBIFICATION UNCLASS IFIED

2b. snoup

3. REPORT TITLE

AN INVESTIGATION OF THE EFFECT OF ACCELERATION

ON THE BURNING RATE OF COMPOSITE PROPELLANTS

4. DESCRIPTIVE NOTES (Typo of mport and incluelvo dateu)

Thes is

5. AUTHOA(S) (Leat neme. Arat name, intllal)

Anderson, James B., LCDR, USN

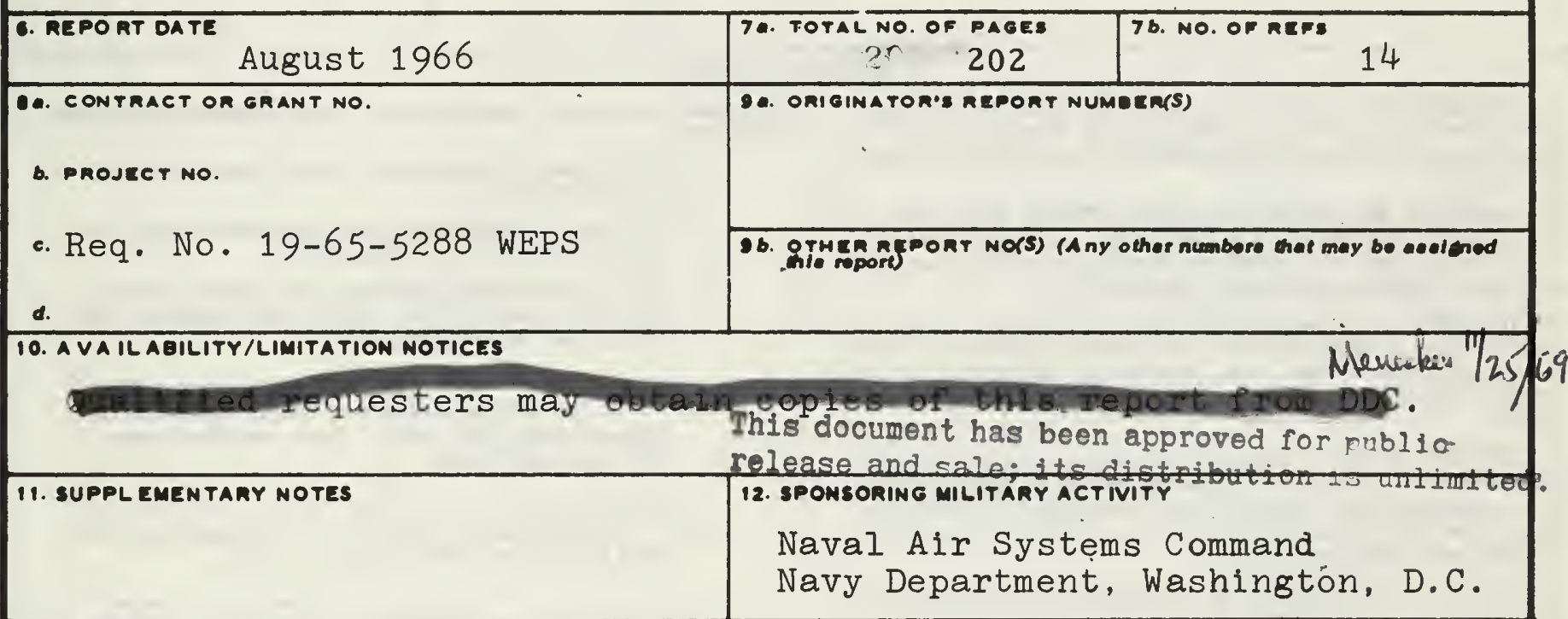

13. ABSTRACT

The average burning rates of composite solid rocket propellants were measured in acceleration fields up to 2000 times the standard acceleration of gravity. The acceleration vector was perpendicular to and into the burning surface. Propellant strands were burned in a combustion bomb mounted on a centrifuge, and surge tanks were employed to ensure essentially constant pressure burning at 500,1000 , and 1500 psia.

The burning rates of both aluminized and non-aluminized composite propellants were found to depend on acceleration. The effect of acceleration on burning rate was found to depend on the burning rate of the propellant without acceleration, aluminum mass loading, and aluminum mass median particle size. The relative burning rate increase was found to be greater for slow burning propellants than for faster burning propeliants.

The experimental results are compared with two models proposed by other investigators. The results indicate that more complex modeling will be required to explain the observed acceleration effects. 


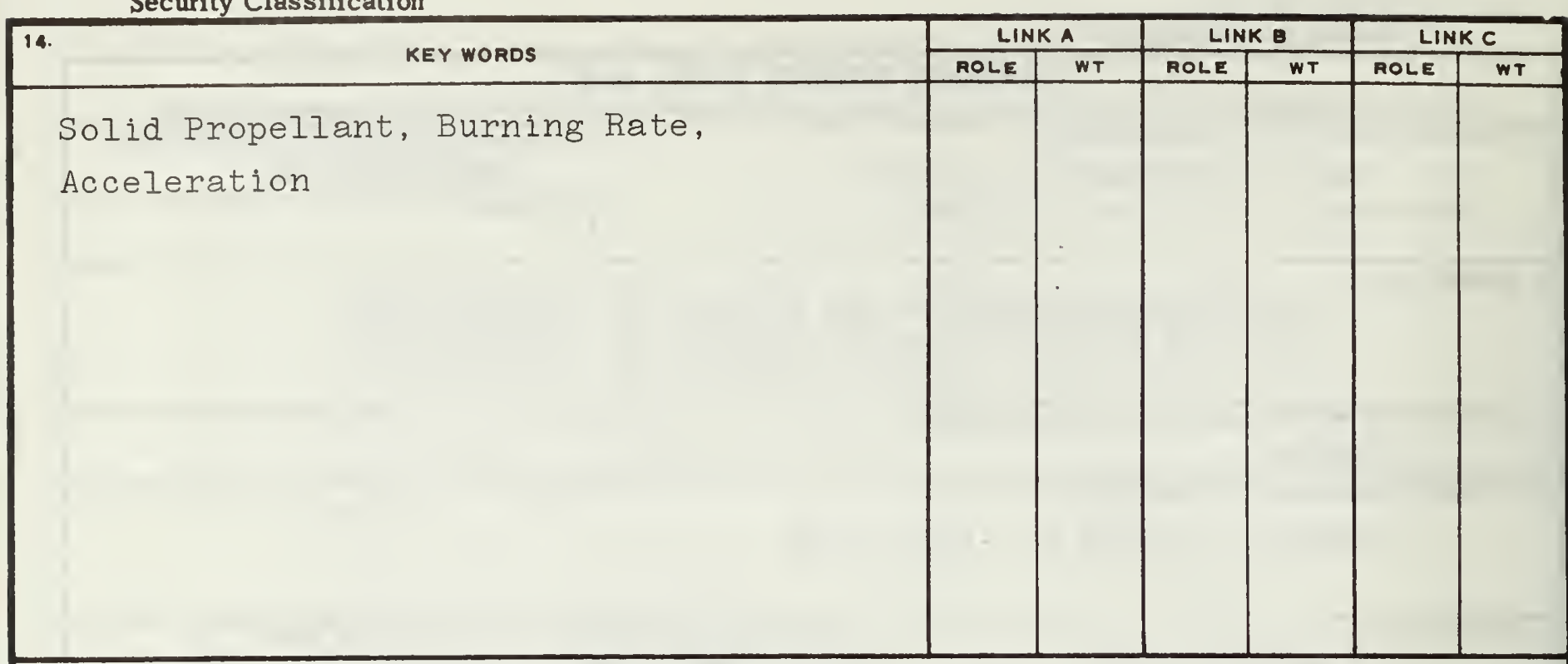

\section{INSTRUCTIONS}

1. ORIGINATING ACTIVITY: Enter the name and address of the contractor, aubcontractor, grantee, Department of Defense activity or other organization (corporate author) is auing the report.

2a. REPORT SECURTY CLASSIFICATION: Enter the overall security classification of the report. Indicate whether "Restricted Data" is included. Marking is to be in accordance with appropriate security regulations.

2b. GROUP: Automatic downerading is specified in DoD Directive 5200.10 and Armed Forces Industrial Manual. Enter the group number. Also, when applicable, show that optional markings have been used for Group 3 and Group 4 as authorized.

3. REPORT TITLE: Enter the complete report title in all capital letters. Titles in all cases should be unclassified. If a meaningful title cannot be selected without classification, show title classification in all capitals in parenthesis immediately following the title.

4. DESCRIPTIVE NOTES: If appropriate, enter the type of report, e.g., interim, progress, summary, annual, or final. Give the inclusive dates when a specific reporting period is covered.

5. AUTHOR(S): Enter the name(s) of author(s) as shown on or in the report. Enter last name, first name, middle initial. If military, show rank and branch of service. The name of the principal author is an absolute minimum requirement.

6. REPORT DATE: Enter the date of the report as day, month, year; or month, year. If more than one date appeara on the report, use date of publication

7a. TOTAL NUMBER OF PAGES: The total page count should follow normal pagination procedures, i. e., enter the number of pages containing information.

7b. NUMBER OF REFERENCES. Enter the total number of references cited in the report.

8a. CONTRACT OR GRANT NUMBER: If appropriate, enter the applicable number of the contract or grant under which the report was written.

$8 b, 8 c, 88 d$. PROJECT NUMBER: Enter the appropriate military department identification, such as project number, subproject number, system numbers, task number, etc.

9a. ORIGINATOR'S REPORT NUMBER(S): Enter the official report number by which the document will be identified and controlled by the originating activity. This number must be unique to this report.

9b. OTHER REPORT NUMBER(S): If the report has been assigned any other report numbers (either by the originator or by the sponsor), al so enter this number(s).

10. AVAIL.ABILITY/LIMITATION NOTICES: Enter any limitations on further dissemination of the report, other than those imposed by security classification, using atandard atatemente such as:

(1) "Qualified requesters may abtain copies of this report from DDC"

(2) "Foreign announcement and diasemination of this report by DDC is not authorized"

(3) "U. S. Government agencies may obtain copiea of this report directly from DDC. Other qualified DDC users shall requeat through

(4) "U. S. military agencies may obtain copies of thit report directly from DDC Other qualified usera shall request through

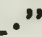

(5) "All distribution of this report is controlled Qualified DDC users shall request through $\therefore$

If the report has been furnished to the Office of Technical Services, Department of Commerce, for sale to the public, indicate this fact and enter the price, if known.

11. SUPPLEMENTARY NOTES: Use for additional explanetory notes.

12. SPONSORING MILITARY ACTIVITY: Ent er the name of the departmental project office or laboratory sponsoring (pay ing for) the research and development. Include addreas.

13. ABSTRACT: Enter an abstract giving a brief and factual summary of the document indicative of the report, even though it may also appear elsewhere in the body of the technical report. If additional space is required, a continuation aheet aball be attached.

It is highly desirable that the abstract of clasified reports be unclassified. Each paragraph of the abstract shall end with an indication of the military security classification of the information in the paragraph, represented as (TS), (S), (C), or (U).

There is no limitation on the length of the abatract. However, the suggested length is from 150 to 225 words.

14. KEY WORDS: Key words are technically meaningful terma or short phrases that characterize a report and may be used a index entries for cataloging the report. Key worda must be selected so that no security classification is required. Identifiers, such as equipment model desionation, trade name, military project code name, geographic location, may be uaed as key words but will be followed by an indication of technical context. The assignment of links, rales, and weights is optional. 


<smiles>[CH]C</smiles> 
DUDLEY KNOX LIBRARY

32768000308530 